\title{
Ring-Expansion Induced 1,2-Metalate Rearrangements: Highly Diastereoselective Synthesis of Cyclobutyl Boronic Esters
}

\author{
Durga Prasad Hari, Joseph C. Abell, Valerio Fasano and Varinder K. Aggarwal* \\ School of Chemistry, University of Bristol, Cantock's Close, Bristol BS8 1TS, U.K. \\ v.aggarwal@bristol.ac.uk.
}

\section{Table of Contents}

$\begin{array}{ll}\text { 1. General methods } & \text { S2 }\end{array}$

$1.1 \quad$ Solvents, Reagents, Glassware and Reaction Setup S2

$\begin{array}{lll}1.2 & \text { Analytical methods } & \text { S2 }\end{array}$

2. Optimization of the reaction conditions $\quad$ S3

$\begin{array}{ll}2.1 \text { Temperature screening } & \text { S3 }\end{array}$

$\begin{array}{ll}2.2 \text { Stoichiometry screening } & \text { S4 }\end{array}$

3. General Procedures $\quad$ S5

3.1 General Procedure A: Synthesis of Vinyl Cyclopropyl Boronic Esters S5

3.2 General Procedure B: Synthesis of Cyclobutyl Boronic Esters S5

3.3 General Procedure C: Synthesis of Cyclobutyl Boronic Esters S6

3.4 General Procedure D: Synthesis of Cyclobutyl Boronic Esters S6

3.5 General Procedure E: Synthesis of Cyclobutyl Boronic Esters $\quad$ S7

3.6 General Procedure F: Synthesis of Cyclobutyl Boronic Esters S8

4. Characterization data $\quad$ S9

5. Stereoselective synthesis of $( \pm)$-grandisol $\quad$ S39

$\begin{array}{ll}\text { 5. DFT Calculations } & \text { S43 }\end{array}$

6. NMR spectra $\quad$ S61

$\begin{array}{ll}\text { 7. Crystal structure and structural assignments } & \text { S117 }\end{array}$

$\begin{array}{ll}\text { 8. GC-MS of } 1 \mathbf{a} \text { and } \mathbf{2 a} & \text { S123 }\end{array}$

$\begin{array}{ll}\text { 9. References } & \text { S124 }\end{array}$ 


\section{General methods}

\subsection{Solvents, Reagents, Glassware and Reaction Setup}

Unless otherwise stated, all reactions were conducted under an inert atmosphere of nitrogen in flame dried glassware using standard Schlenk techniques. Air- and moisture-sensitive liquids and solutions were transferred via syringe into the reaction vessels through rubber septa. Unless otherwise specified, all reagents were purchased at highest commercial quality and used as received. Non-anhydrous solvents were purchased (unless specified) at the highest commercial quality and used as received. Anhydrous DMF, and methanol were purchased from Acros and used as received. DCM and THF were dried on an Anhydrous Engineering alumina column drying system. Temperatures described below $-10{ }^{\circ} \mathrm{C}$ were achieved using Thermo Scientific EK-90 or Huber TC100E cryostats or appropriate solvent/dry ice baths.

\subsection{Analytical methods}

Chromatography: Flash column chromatography was carried out using Sigma-Aldrich silica gel (60 ̊, 230-400 mesh, 40-63 $\mu \mathrm{m}$ ) or a Biotage IsoleraTM flash purification system. Reactions were followed by thin-layer chromatography (TLC) where practical, using aluminium-backed Merck Kieselgel 60 F254 fluorescent treated silica gel plates, which were visualised under UV light or by staining with aqueous basic potassium permanganate, acidic $p$-anisaldehyde solution in ethanol, or phosphomolybdic acid solution in ethanol. NMR yields: Following work up, 1,3,5 trimethoxybenzene (1 equiv relative to limiting SM) was added to the crude residue. The resultant mixture was dissolved in $\mathrm{CDCl}_{3}(5 \mathrm{~mL})$, and a $0.6 \mathrm{~mL}$ sample of the resultant solution taken for ${ }^{1} \mathrm{H}$ NMR analysis. Yields were calculated based on the integrals of known product resonances relative to the 1,3,5 TMB standard resonance $(9 \mathrm{H}$, at $3.77 \mathrm{ppm}$ in $\mathrm{CDCl}_{3}$ ). IR: IR spectra were recorded on neat compounds using a Perkin Elmer (Spectrum One) FT-IR spectrometer (ATR sampling accessory). Selected absorbances (vmax, expressed in $\mathrm{cm}^{-1}$ ) are reported and denoted w. (weak), m. (medium) or s. (strong). ${ }^{\mathbf{1}} \mathbf{H}$ NMR: Spectra were recorded on Jeol ECS (400 MHz), Jeol ECZ (400 MHz or Bruker Avance (400 $\mathrm{MHz}$ or $500 \mathrm{MHz}$ ) instruments. Chemical shifts $(\delta)$ are quoted in parts per million (ppm) and referenced to the appropriate NMR solvent peak(s) and are assigned in accordance with numbered diagrams; with resonances described as s (singlets), $\mathrm{d}$ (doublets), $\mathrm{t}$ (triplets), $\mathrm{q}$ (quartets), p (pentets), combinations thereof (i.e. td indicates a triplet of doublets) or $\mathrm{m}$ (multiplets) and br s (broad singlet). ${ }^{13} \mathbf{C}$ NMR: Spectra were recorded on a Bruker Avance 
(101 MHz or $126 \mathrm{MHz}$ ) instrument. Chemical shifts $(\delta)$ are quoted in parts per million (ppm) and referenced to the appropriate NMR solvent peak(s) and are assigned in accordance with numbered diagrams. HRMS: High resolution mass spectra were recorded on a Bruker Daltronics MicroTOF II (ESI), Thermo Scientific Orbitrap (ESI, APCI) or Thermo Scientific QExactive (EI). Only molecular ion $\left([\mathrm{M}+\mathrm{H}]^{+}\right.$or $[\mathrm{M}+\mathrm{Na}]^{+}$for ESI and APCI; $\mathrm{M}^{+}$for EI peaks are reported.

\section{Optimization of the reaction conditions}

\subsection{Temperature screening}

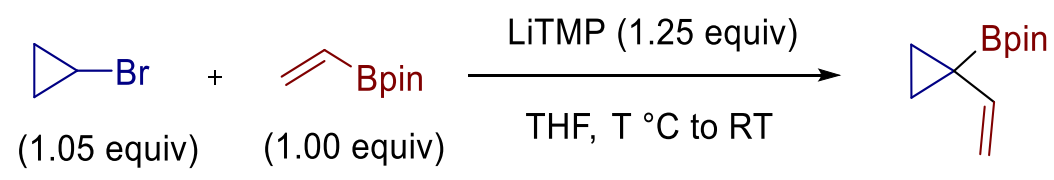

Following a modified procedure, ${ }^{1}$ to a stirred solution of 2,2,6,6-tetramethylpiperidine (190 $\mu \mathrm{L}, 156 \mathrm{mg}, 1.25 \mathrm{mmol}, 1.25$ equiv) in $\mathrm{THF}(1.5 \mathrm{M})$ under $\mathrm{N}_{2}$ at $\mathrm{T}_{1}{ }^{\circ} \mathrm{C}$ was added $n \mathrm{BuLi}(0.700$ $\mathrm{mL}, 1.25 \mathrm{mmol}, 1.25$ equiv) dropwise over $15 \mathrm{~min}$. The solution was then stirred for $30 \mathrm{~min}$ at $\mathrm{T}_{1}{ }^{\circ} \mathrm{C}$, warmed to $\mathrm{T}_{2}{ }^{\circ} \mathrm{C}$ and stirred for a further $30 \mathrm{~min}$. In another Schlenk flask, a solution of cyclopropyl bromide ( $88.0 \mu \mathrm{L}, 1.05 \mathrm{mmol}, 1.05$ equiv) and vinyl boronic ester (170 $\mu \mathrm{L}, 1.00$ mmol, 1.00 equiv) was prepared in THF $(0.4 \mathrm{M})$ and cooled to $\mathrm{T}_{3}{ }^{\circ} \mathrm{C}$. To this solution was added the freshly prepared LiTMP over $20 \mathrm{~min}$. The reaction was allowed to stir at $\mathrm{T}_{3}{ }^{\circ} \mathrm{C}$ for 2 $\mathrm{h}$, then allowed to warm slowly to RT overnight. A saturated solution of $\mathrm{NaHCO}_{3}(15 \mathrm{~mL})$ was added to quench the reaction, and the mixture was allowed to warm to RT. Diethyl ether (15 $\mathrm{mL}$ ) was added and the layers were separated. The aqueous phase was extracted with ether (3 $\mathrm{x} 15 \mathrm{~mL})$ and the combined organic layers were washed with water $(20 \mathrm{~mL})$ and brine $(20 \mathrm{~mL})$, dried over $\mathrm{MgSO}_{4}$, filtered and concentrated to afford the crude product. Crude material was purified by flash column chromatography (DCM/pentane 1;20) to afford the desired product.

\begin{tabular}{ccccc}
\hline Entry & $\mathbf{T}_{\mathbf{1}}$ & $\mathbf{T}_{\mathbf{2}}$ & $\mathbf{T}_{\mathbf{3}}$ & Yield \\
\hline 1 & $-78{ }^{\circ} \mathrm{C}$ & $0{ }^{\circ} \mathrm{C}$ & $-95{ }^{\circ} \mathrm{C}$ & $<5 \%$ \\
2 & $-78{ }^{\circ} \mathrm{C}$ & $0{ }^{\circ} \mathrm{C}$ & $-78{ }^{\circ} \mathrm{C}$ & $<5 \%$ \\
3 & $0{ }^{\circ} \mathrm{C}$ & $\mathrm{RT}$ & $-78{ }^{\circ} \mathrm{C}$ & $<5 \%$ \\
4 & $\mathbf{0}{ }^{\circ} \mathrm{C}$ & $\mathrm{RT}$ & $0{ }^{\circ} \mathrm{C}$ & $\mathbf{6 3 \%}$ \\
5 & $0{ }^{\circ} \mathrm{C}$ & $\mathrm{RT}$ & $\mathrm{RT}$ & $47 \%$ \\
\hline
\end{tabular}




\subsection{Stoichiometry screening}

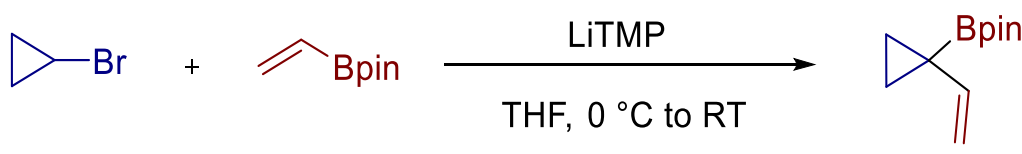

To a stirred solution of 2,2,6,6-tetramethylpiperidine (x mmol, $\mathrm{x}$ equiv) in THF (1.5 M) under $\mathrm{N}_{2}$ at $0{ }^{\circ} \mathrm{C}$ was added $n \mathrm{BuLi}$ (x mmol, x equiv) dropwise over $15 \mathrm{~min}$. The solution was then stirred for $30 \mathrm{~min}$ at $0{ }^{\circ} \mathrm{C}$, warmed to RT and stirred for a further $30 \mathrm{~min}$. In another Schlenk flask, a solution of cyclopropyl bromide (y mmol, y equiv) and vinyl boronic ester (1.00 mmol, 1.00 equiv) was prepared in THF $(0.4 \mathrm{M})$ and cooled to $0{ }^{\circ} \mathrm{C}$. To this solution was added the freshly prepared LiTMP over $20 \mathrm{~min}$. The reaction was allowed to stir at $0{ }^{\circ} \mathrm{C}$ for $2 \mathrm{~h}$, then allowed to warm slowly to RT overnight. A saturated solution of $\mathrm{NaHCO}_{3}(15 \mathrm{~mL})$ was added to quench the reaction, and the mixture was allowed to warm to RT. Diethyl ether $(15 \mathrm{~mL})$ was added and the layers were separated. The aqueous phase was extracted with ether $(3 \mathrm{x} 15 \mathrm{~mL})$ and the combined organic layers were washed with water $(20 \mathrm{~mL})$ and brine $(20 \mathrm{~mL})$, dried over $\mathrm{MgSO}_{4}$, filtered and concentrated to afford the crude product. Crude material was purified by flash column chromatography (DCM/pentane 1;20) to afford the desired product.

\begin{tabular}{ccccc}
\hline Entry & TMPH (x equiv) & ${ }^{n}$ BuLi (x equiv) & $D-\mathrm{Br}_{(\mathbf{y} \text { equiv) }}$ & Yield \\
\hline 1 & 1.25 & 1.25 & 1.05 & $63 \%$ \\
2 & 1.50 & 1.50 & 1.50 & $56 \%$ \\
3 & 1.10 & 1.10 & 1.50 & $72 \%$ \\
4 & $\mathbf{1 . 1 0}$ & $\mathbf{1 . 1 0}$ & $\mathbf{1 . 1 0}$ & $\mathbf{7 2 \%}$ \\
\hline
\end{tabular}




\section{General Procedures}

\subsection{General Procedure A: Synthesis of Vinyl Cyclopropyl Boronic Esters}

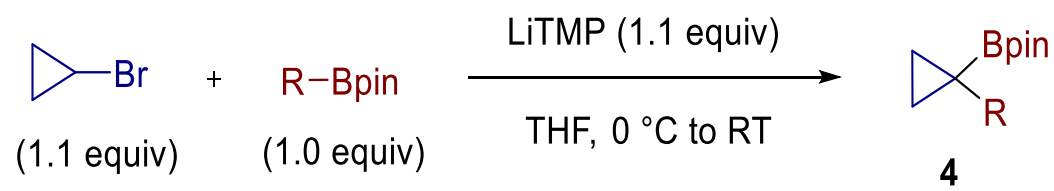

To a stirred solution of 2,2,6,6-tetramethylpiperidine (5.50 mmol, 1.10 equiv) in THF (1.5 M) under $\mathrm{N}_{2}$ at $0{ }^{\circ} \mathrm{C}$ was added $n \operatorname{BuLi}(5.50 \mathrm{mmol}, 1.10$ equiv) dropwise over $15 \mathrm{~min}$. The solution was then stirred for $30 \mathrm{~min}$ at $0{ }^{\circ} \mathrm{C}$, warmed to $\mathrm{RT}$ and stirred for a further $30 \mathrm{~min}$. In another Schlenk flask, a solution of cyclopropyl bromide (5.50 mmol, 1.10 equiv) and vinyl boronic ester (5.00 mmol, 1.00 equiv) was prepared in THF $(0.4 \mathrm{M})$ and cooled to $0{ }^{\circ} \mathrm{C}$. To this solution was added the freshly prepared LiTMP over $20 \mathrm{~min}$. The reaction was allowed to stir at $0{ }^{\circ} \mathrm{C}$ for $2 \mathrm{~h}$, then allowed to warm slowly to RT overnight. A saturated solution of $\mathrm{NaHCO}_{3}(15$ $\mathrm{mL}$ ) was added to quench the reaction, and the mixture was allowed to warm to RT. Diethyl ether $(15 \mathrm{~mL})$ was added and the layers were separated. The aqueous phase was extracted with ether $(3 \times 15 \mathrm{~mL})$ and the combined organic layers were washed with water $(20 \mathrm{~mL})$ and brine (20 mL), dried over $\mathrm{MgSO}_{4}$, filtered and concentrated to afford the crude product. Crude material was purified by flash column chromatography (DCM/pentane or $\mathrm{Et}_{2} \mathrm{O}$ :pentane) to afford the desired product.

\subsection{General Procedure B: Synthesis of Cyclobutyl Boronic Esters}

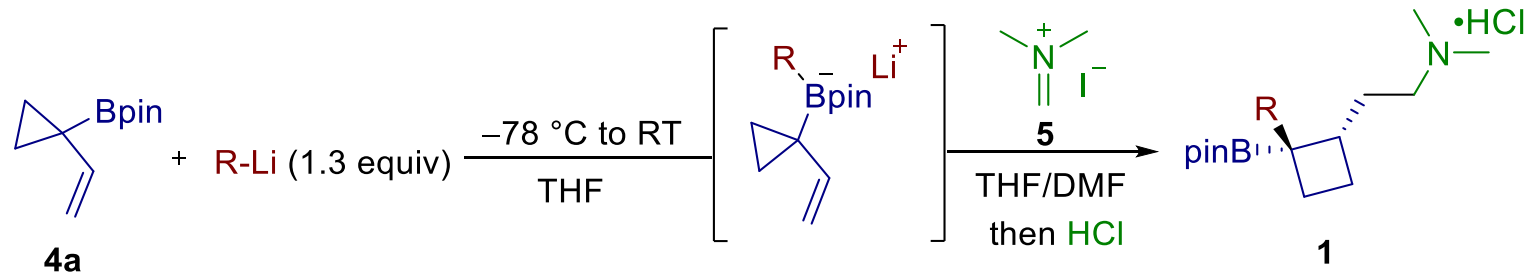

To a solution of vinyl cyclopropyl boronic ester $\mathbf{4 a}(0.300 \mathrm{mmol}, 1.00$ equiv) in THF (2 mL) under $\mathrm{N}_{2}$ at $-78^{\circ} \mathrm{C}$ was added organolithium ( $0.390 \mathrm{mmol}, 1.30$ equiv) dropwise. The reaction mixture was stirred at $-78^{\circ} \mathrm{C}$ for $1 \mathrm{~h}$ and then warmed to RT and stirred for another $1 \mathrm{~h}$. The reaction mixture was cooled back to $-40{ }^{\circ} \mathrm{C}$ and DMF (2 mL) was added. Eschenmoser's salt (5) $\left(0.600 \mathrm{mmol}, 2.00\right.$ equiv) was then added as a solid at $-40{ }^{\circ} \mathrm{C}$. The reaction was allowed to stir at $-40{ }^{\circ} \mathrm{C}$ for $2 \mathrm{~h}$, then allowed to warm slowly to RT overnight. $0.5 \mathrm{~N} \mathrm{NaOH}(20 \mathrm{~mL})$ was 
then added, and the mixture was extracted with $\mathrm{Et}_{2} \mathrm{O}(3 \times 20 \mathrm{~mL})$. The combined organic phases were dried over $\mathrm{MgSO}_{4}$, filtered, and concentrated under reduced pressure. The crude material was then dissolved in the minimum amount of $\mathrm{Et}_{2} \mathrm{O} /$ penatne $(\mathrm{ca} .0 .6 \mathrm{~mL}$ ) and then $2 \mathrm{~N} \mathrm{HCl}$ ( 0.330 mmol, 1.10 equiv) in $\mathrm{Et}_{2} \mathrm{O}$ was added. A precipitate was formed, which was collected via a suction filtration (pentane was used for transfer of the compound) and then thoroughly dried under high vacuum.

\subsection{General Procedure C: Synthesis of Cyclobutyl Boronic Esters}

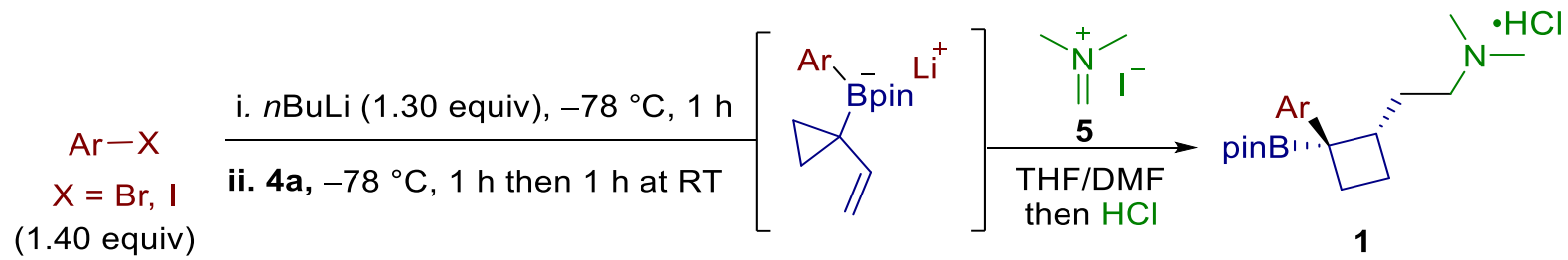

To a stirred solution of arylhalide $\left(0.420 \mathrm{mmol}, 1.40\right.$ equiv) in THF $(1 \mathrm{~mL})$ under $\mathrm{N}_{2}$ at $-78{ }^{\circ} \mathrm{C}$ was added $n \mathrm{BuLi}(0.390 \mathrm{mmol}, 1.30$ equiv) dropwise. The solution was then stirred for $1 \mathrm{~h}$ at $-78{ }^{\circ} \mathrm{C}$, at which point a solution of vinyl cyclopropyl boronic ester ( $0.300 \mathrm{mmol}, 1.00$ equiv) in THF $(1 \mathrm{~mL})$ was added dropwise. The reaction mixture was stirred at $-78{ }^{\circ} \mathrm{C}$ for $1 \mathrm{~h}$ and then warmed to RT and stirred for another $1 \mathrm{~h}$. The reaction mixture was cooled back to -40 ${ }^{\circ} \mathrm{C}$ and DMF (2 mL) was added. Eschenmoser's salt (5) (0.600 mmol, 2.00 equiv) was then added as a solid at $-40{ }^{\circ} \mathrm{C}$. The reaction was allowed to stir at $-40{ }^{\circ} \mathrm{C}$ for $2 \mathrm{~h}$, then allowed to warm slowly to RT overnight. $0.5 \mathrm{~N} \mathrm{NaOH}(20 \mathrm{~mL})$ was then added, and the mixture was extracted with $\mathrm{Et}_{2} \mathrm{O}(3 \times 20 \mathrm{~mL})$. The combined organic phases were dried over $\mathrm{MgSO}_{4}$, filtered, and concentrated under reduced pressure. The crude material was then dissolved in the minimum amount of $\mathrm{Et}_{2} \mathrm{O} /$ penatne (ca. $\left.0.6 \mathrm{~mL}\right)$ and then $2 \mathrm{~N} \mathrm{HCl}(0.330 \mathrm{mmol}, 1.10$ equiv) in $\mathrm{Et}_{2} \mathrm{O}$ was added. A precipitate was formed, which was collected via a suction filtration (pentane was used for transfer of the compound) and then thoroughly dried under high vacuum.

\subsection{General Procedure D: Synthesis of Cyclobutyl Boronic Esters}

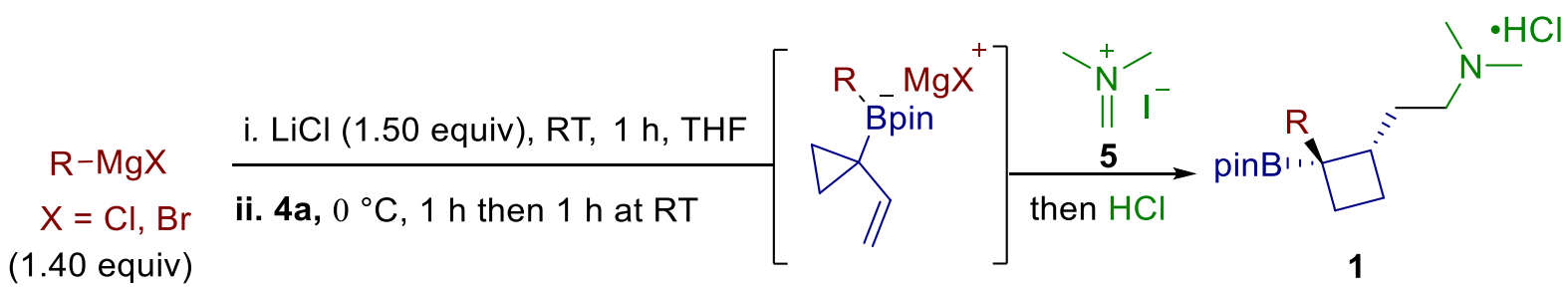


To a stirred solution of $\mathrm{LiCl}(19.0 \mathrm{mg}, 0.450 \mathrm{mmol}, 1.50$ equiv) in THF ( $2 \mathrm{~mL})$ under $\mathrm{N}_{2}$ at RT was added $\mathrm{RMgX}(0.390 \mathrm{mmol}, 1.30$ equiv $)$ dropwise. The solution was stirred for $1 \mathrm{~h}$ and then cooled back to $0{ }^{\circ} \mathrm{C}$, at which point a solution of vinyl cyclopropyl boronic ester $(0.300$ mmol, 1.00 equiv. $)$ in THF ( $2 \mathrm{~mL})$ was added dropwise. The reaction mixture was stirred at 0 ${ }^{\circ} \mathrm{C}$ for $1 \mathrm{~h}$ and then warmed to RT and stirred for another $1 \mathrm{~h}$. The reaction mixture was cooled back to $-78{ }^{\circ} \mathrm{C}$ and Eschenmoser's salt (5) (0.600 mmol, 2.00 equiv) was added. The reaction was allowed to stir at $-78{ }^{\circ} \mathrm{C}$ for $2 \mathrm{~h}$, then allowed to warm slowly to RT overnight. $0.5 \mathrm{~N}$ $\mathrm{NaOH}(20 \mathrm{~mL})$ was then added, and the mixture was extracted with $\mathrm{Et}_{2} \mathrm{O}(3 \times 20 \mathrm{~mL})$. The combined organic phases were dried over $\mathrm{MgSO}_{4}$, filtered, and concentrated under reduced pressure. The crude material was then dissolved in the minimum amount of $\mathrm{Et}_{2} \mathrm{O}$ /penatne (ca. $0.6 \mathrm{~mL})$ and then $2 \mathrm{~N} \mathrm{HCl}\left(0.330 \mathrm{mmol}, 1.10\right.$ equiv) in $\mathrm{Et}_{2} \mathrm{O}$ was added. A precipitate was formed, which was collected via a suction filtration (pentane was used for transfer of the compound) and then thoroughly dried under high vacuum.

\subsection{General Procedure E: Synthesis of Cyclobutyl Boronic Esters}

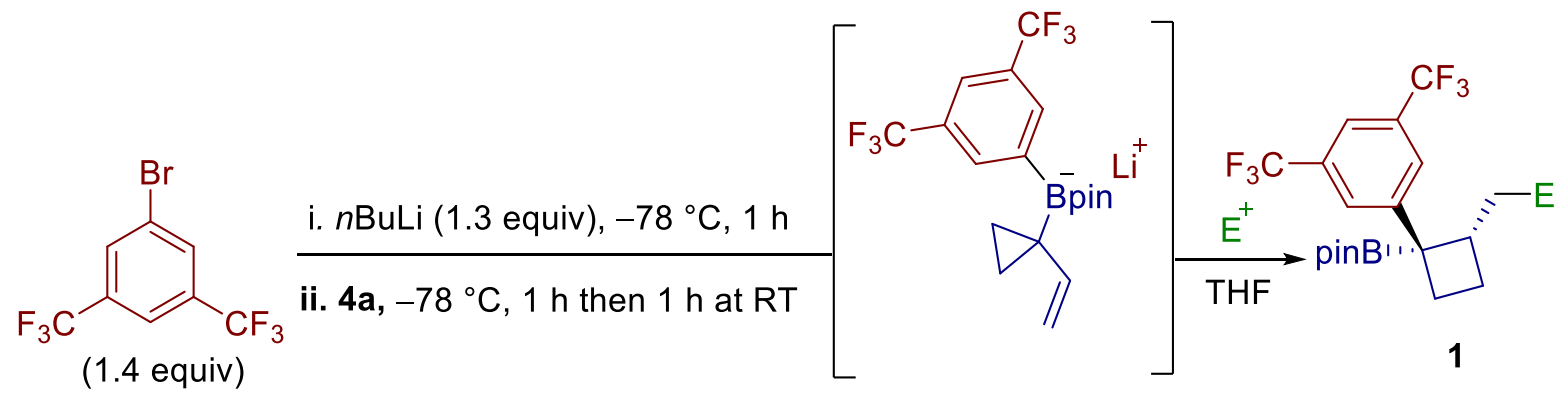

To a stirred solution of 1-bromo-3,5-bis(trifluoromethyl)benzene ( $0.420 \mathrm{mmol}, 1.40$ equiv) in THF ( $2 \mathrm{~mL})$ under $\mathrm{N}_{2}$ at $-78{ }^{\circ} \mathrm{C}$ was added $n \mathrm{BuLi}$ ( $0.390 \mathrm{mmol}, 1.30$ equiv) dropwise. The solution was then stirred for $1 \mathrm{~h}$ at $-78{ }^{\circ} \mathrm{C}$, at which point a solution of vinyl cyclopropyl boronic ester $(0.300 \mathrm{mmol}, 1.00$ equiv) in THF $(2 \mathrm{~mL})$ was added dropwise. The reaction mixture was stirred at $-78{ }^{\circ} \mathrm{C}$ for $1 \mathrm{~h}$ and then warmed to RT and stirred for another $1 \mathrm{~h}$. The reaction mixture was cooled back to $-78{ }^{\circ} \mathrm{C}$ and electrophile $(0.600 \mathrm{mmol}, 2.00$ equiv) was added as a solid. The reaction was allowed to stir at $-78{ }^{\circ} \mathrm{C}$ for $2 \mathrm{~h}$, then allowed to warm slowly to RT overnight. $0.5 \mathrm{~N} \mathrm{NaOH}(20 \mathrm{~mL})$ was then added, and the mixture was extracted with $\mathrm{Et}_{2} \mathrm{O}(3 \times 20 \mathrm{~mL})$. The combined organic phases were dried over $\mathrm{MgSO}_{4}$, filtered, and concentrated under reduced pressure. The crude material was purified by flash chromatography using DCM/MeOH or $\mathrm{Et}_{2} \mathrm{O} /$ pentane as mobile phase to afford $\mathbf{1}$. 


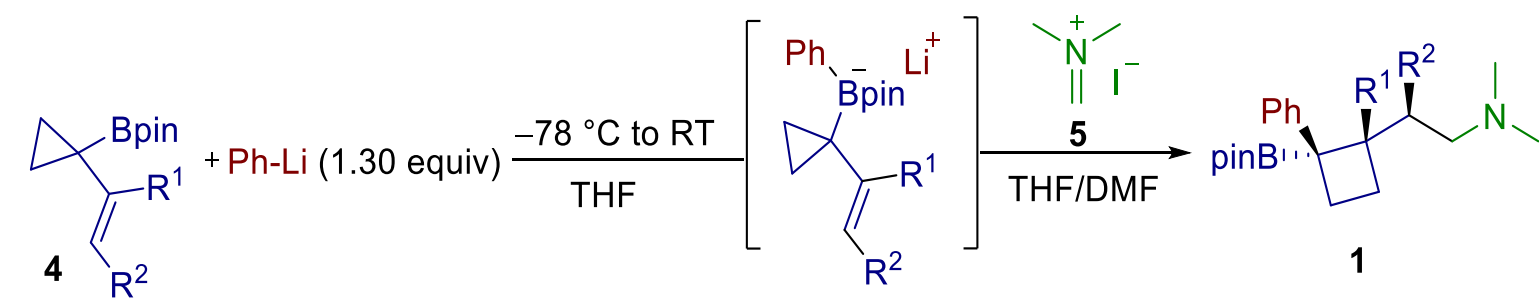

To a solution of vinyl cyclopropyl boronic ester 4 (0.300 mmol, 1.00 equiv) in THF ( $2 \mathrm{~mL})$ under $\mathrm{N}_{2}$ at $-78^{\circ} \mathrm{C}$ was added $\mathrm{PhLi}(206 \mu \mathrm{L}, 0.390 \mathrm{mmol}, 1.9 \mathrm{M}$ in in dibutyl ether, 1.30 equiv $)$ dropwise. The reaction mixture was stirred at $-78^{\circ} \mathrm{C}$ for $1 \mathrm{~h}$ and then warmed to RT and stirred for another $1 \mathrm{~h}$. The reaction mixture was cooled back to $-40{ }^{\circ} \mathrm{C}$ and DMF (2 mL) was added. Eschenmoser's salt (5) (0.600 mmol, 2.00 equiv) was then added as a solid at $-40{ }^{\circ} \mathrm{C}$. The reaction was allowed to stir at $-40{ }^{\circ} \mathrm{C}$ for $2 \mathrm{~h}$, then allowed to warm slowly to RT overnight. $0.5 \mathrm{~N} \mathrm{NaOH}(20 \mathrm{~mL})$ was then added, and the mixture was extracted with $\mathrm{Et}_{2} \mathrm{O}(3 \times 20 \mathrm{~mL})$. The combined organic phases were dried over $\mathrm{MgSO}_{4}$, filtered, and concentrated under reduced pressure. The crude material was purified by flash chromatography using DCM/MeOH as mobile phase to afford $\mathbf{1}$. 


\section{Characterization data}

\section{4,4,5,5-Tetramethyl-2-(1-vinylcyclopropyl)-1,3,2-dioxaborolane (4a)}

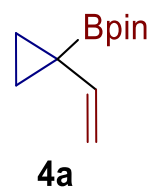

Following general procedure A, using 2,2,6,6-tetramethylpiperidine (770 mg, $930 \mu \mathrm{L}, 5.50$ mmol, 1.10 equiv), ${ }^{n} \mathrm{BuLi}$ (3.44 mL, $5.50 \mathrm{mmol}, 1.60 \mathrm{M}$ in hexanes, 1.10 equiv), cyclopropyl bromide (665 mg, $440 \mu \mathrm{L}, 5.50 \mathrm{mmol}, 1.00$ equiv) and 4,4,5,5-tetramethyl-2-vinyl-1,3,2dioxaborolane ( $770 \mathrm{mg}, 5.00 \mathrm{mmol}, 1.00$ equiv). The crude reaction mixture was purified by flash chromatography using DCM:pentane 1:10 as mobile phase to afford 4a (704 mg, 3.63 mmol, 72\%) as a colorless liquid (highly volatile, solvents removed at RT). TLC (DCM:pentane, 1:5 v/v): $\mathrm{R}_{\mathrm{f}}=0.26, \mathrm{KMnO}_{4} ;{ }^{1} \mathrm{H} \mathrm{NMR}\left(400 \mathrm{MHz}, \mathrm{CDCl}_{3}\right): \delta 5.78(\mathrm{dd}, J=17.3$, $\left.10.5 \mathrm{~Hz}, 1 \mathrm{H}, \mathrm{CH}=\mathrm{CH}_{2}\right), 5.10\left(\mathrm{dd}, J=17.3,1.7 \mathrm{~Hz}, 1 \mathrm{H}, \mathrm{C}=\mathrm{CH}^{a} \mathrm{H}^{\mathrm{b}}\right), 4.87(\mathrm{dd}, J=10.5,1.7 \mathrm{~Hz}$, $\left.1 \mathrm{H}, \mathrm{C}=\mathrm{CH}^{\mathrm{a}} H^{b}\right), 1.22(\mathrm{~s}, 12 \mathrm{H}, \mathrm{Bpin}), 0.99-0.90(\mathrm{~m}, 2 \mathrm{H}), 0.72-0.62(\mathrm{~m}, 2 \mathrm{H}) ;{ }^{13} \mathrm{C} \mathrm{NMR}(100$ $\mathrm{MHz}_{\mathrm{CDCl}}$ ): $\delta$ 142.4, 111.6, 83.1, 24.7, 14.0; IR v 2978 (s), 2927 (m), 1633 (m), 1421 (m), 1388 (s), 1317 (m), 1139 (s), 1046 (w), 854 (m); HRMS (APCI) calcd. for $\mathrm{C}_{11} \mathrm{H}_{20} \mathrm{BO}_{2}{ }^{+}[\mathrm{M}+\mathrm{H}]^{+}$ 195.1551; found 195.1547. Carbon attached to boron not observed due to quadrupolar relaxation. See spectra

\section{(E)-4,4,5,5-Tetramethyl-2-(1-(prop-1-en-1-yl)cyclopropyl)-1,3,2-dioxaborolane (4b)}

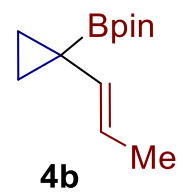

Following general procedure A on a $2.5 \mathrm{mmol}$ scale, using 2,2,6,6-tetramethylpiperidine (385 $\mathrm{mg}, 465 \mu \mathrm{L}, 2.75 \mathrm{mmol}, 1.10$ equiv), $n \mathrm{BuLi}$ (1.75 mL, $2.75 \mathrm{mmol}, 1.60 \mathrm{M}$ in hexanes, 1.10 equiv), cyclopropyl bromide (333 $\mathrm{mg}, 220 \mu \mathrm{L}, 2.75 \mathrm{mmol}, 1.00$ equiv) and (E)-4,4,5,5tetramethyl-2-(prop-1-en-1-yl)-1,3,2-dioxaborolane (420 mg, $2.50 \mathrm{mmol}, 1.00$ equiv). The crude reaction mixture was purified by flash chromatography using DCM:pentane 1:10 as mobile phase to afford $\mathbf{4 b}(289 \mathrm{mg}, 1.39 \mathrm{mmol}, 56 \%)$ as a colourless liquid. Extensive exposure to vacuum was avoided due to concerns about product volatility. TLC (DCM:pentane, 1:5 v/v): $\mathrm{R}_{\mathrm{f}}=0.27, \mathrm{KMnO}_{4} ;{ }^{1} \mathrm{H} \mathrm{NMR}\left(400 \mathrm{MHz}, \mathrm{CDCl}_{3}\right): \delta 5.53(\mathrm{dq}, J=15.4,6.4 \mathrm{~Hz}, 1 \mathrm{H}$, 
$\left.\mathrm{CH}=\mathrm{CHCH}_{3}\right), 5.37\left(\mathrm{dd}, J=15.4,6.4 \mathrm{~Hz}, 1 \mathrm{H}, \mathrm{CH}=\mathrm{CHCH}_{3}\right), 1.63(\mathrm{dd}, J=6.4,1.5 \mathrm{~Hz}, 3 \mathrm{H}$, $\left.\mathrm{CH}=\mathrm{CHCH}_{3}\right), 1.22$ (s, 12H, Bpin), $0.88(\mathrm{ddd}, J=3.4,3.4,3.4 \mathrm{~Hz}, 2 \mathrm{H}), 0.60$ (ddd, $J=3.4,3.4$, $3.4 \mathrm{~Hz}, 2 \mathrm{H}) ;{ }^{13} \mathrm{C} \mathrm{NMR}\left(100 \mathrm{MHz}, \mathrm{CDCl}_{3}\right): \delta 135.0,122.4,83.2,24.8,18.2,13.9$; IR v 2979 (m), $1730(\mathrm{~m}), 1372(\mathrm{w}), 1321(\mathrm{w}), 1140$ (s), 854 (w); HRMS (EI) calcd. for $\mathrm{C}_{12} \mathrm{H}_{21} \mathrm{BO}_{2}{ }^{+}[\mathrm{M}]^{+}$ 208.1629; found: 208.1628. Carbon attached to boron not observed due to quadrupolar relaxation. $\underline{\text { See spectra }}$

\section{(E)-2-(1-(Hex-1-en-1-yl)cyclopropyl)-4,4,5,5-tetramethyl-1,3,2-dioxaborolane (4c)}

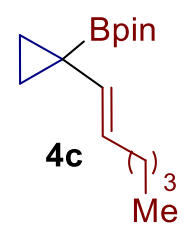

Following general procedure A, using 2,2,6,6-tetramethylpiperidine (770 mg, $930 \mu \mathrm{L}, 5.50$ mmol, 1.10 equiv), $n \mathrm{BuLi}$ (3.44 mL, $5.50 \mathrm{mmol}, 1.60 \mathrm{M}$ in hexanes, 1.10 equiv), cyclopropyl bromide (665 mg, $440 \mu \mathrm{L}, 5.50 \mathrm{mmol}, 1.00$ equiv) and (E)-2-(hex-1-en-1-yl)-4,4,5,5tetramethyl-1,3,2-dioxaborolane ( $1.05 \mathrm{~g}, 5.00 \mathrm{mmol}, 1.00$ equiv). The crude reaction mixture was purified by flash chromatography using DCM:pentane 1:10 as mobile phase to afford $\mathbf{4 c}$ (924 mg, $3.70 \mathrm{mmol}, 74 \%$ ) as a colorless liquid. TLC (DCM:pentane, 1:5 v/v): $\mathrm{R}_{\mathrm{f}}=0.28$, $\mathrm{KMnO}_{4} ;{ }^{1} \mathrm{H} \mathrm{NMR}\left(400 \mathrm{MHz}, \mathrm{CDCl}_{3}\right): \delta 5.48\left(\mathrm{dt}, J=15.4,6.5 \mathrm{~Hz}, 1 \mathrm{H}, \mathrm{CH}=\mathrm{CHCH}_{2}\right), 5.38(\mathrm{~d}$, $\left.J=15.5 \mathrm{~Hz}, 1 \mathrm{H}, \mathrm{CH}=\mathrm{CHCH}_{2}\right), 2.01-1.87\left(\mathrm{~m}, 2 \mathrm{H}, \mathrm{CH}=\mathrm{CHCH}_{2}\right), 1.34-1.24(\mathrm{~m}, 4 \mathrm{H}$, $\mathrm{CH}_{2} \mathrm{CH}_{2} \mathrm{CH}_{3}$ ), 1.22 (s, 12H, Bpin), $0.89-0.84$ (m, 5H), $0.67-0.53$ (m, 2H); ${ }^{13} \mathrm{C}$ NMR (100 $\left.\mathrm{MHz}, \mathrm{CDCl}_{3}\right): \delta$ 133.6, 127.7, 83.0, 32.4, 32.0, 24.7, 22.2, 14.0, 14.0; IR v 2977 (m), 2925 (m), 1404 (m), 1315 (m), 1138 (s), 967 (m), 854 (w); HRMS (ESI) calcd. for $\mathrm{C}_{15} \mathrm{H}_{31} \mathrm{BNO}_{2}{ }^{+}$ $\left[\mathrm{M}+\mathrm{NH}_{4}\right]^{+}$268.2445; found 268.2437. Carbon attached to boron not observed due to quadrupolar relaxation. See spectra

(E)-Tert-butyldimethyl((4-(1-(4,4,5,5-tetramethyl-1,3,2-dioxaborolan-2yl)cyclopropyl)but-3-en-1-yl)oxy)silane (4d)

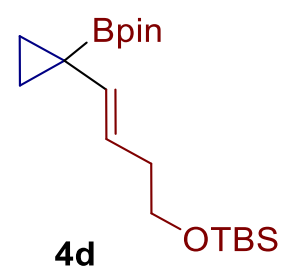


Following general procedure A on a $2.5 \mathrm{mmol}$ scale, using 2,2,6,6-tetramethylpiperidine (385 $\mathrm{mg}, 465 \mu \mathrm{L}, 2.75 \mathrm{mmol}, 1.10$ equiv), $n \mathrm{BuLi}$ (1.75 mL, $2.75 \mathrm{mmol}, 1.60 \mathrm{M}$ in hexanes, 1.10 equiv), cyclopropyl bromide (333 mg, $220 \mu \mathrm{L}, 2.75 \mathrm{mmol}, 1.00$ equiv) and (E)-tertbutyldimethyl((4-(4,4,5,5-tetramethyl-1,3,2-dioxaborolan-2-yl)but-3-en-1-yl)oxy)silane (781 $\mathrm{mg}, 2.50 \mathrm{mmol}, 1.00$ equiv). The crude reaction mixture was purified by flash chromatography using 1:10 DCM:petroleum ether to 100\% DCM as mobile phase to afford 4d (646 mg, 1.82 mmol, 73\%) as a colourless liquid. TLC (DCM:pentane, $1: 5 \mathrm{v} / \mathrm{v}): \mathrm{R}_{\mathrm{f}}=0.31, \mathrm{KMnO}_{4} ;{ }^{1} \mathrm{H}$ NMR $\left(400 \mathrm{MHz}, \mathrm{CDCl}_{3}\right): \delta 5.52\left(\mathrm{dt}, J=15.5,6.9 \mathrm{~Hz}, 1 \mathrm{H}, \mathrm{CH}=\mathrm{CHCH}_{2}\right), 5.40(\mathrm{dt}, J=15.5,1.1 \mathrm{~Hz}$, $\left.1 \mathrm{H}, \mathrm{CH}=\mathrm{CHCH}_{2}\right), 3.58\left(\mathrm{t}, J=6.9 \mathrm{~Hz}, 2 \mathrm{H}, \mathrm{CH}_{2} \mathrm{CH}_{2} \mathrm{O}\right), 2.19(\mathrm{qd}, J=6.9,1.1 \mathrm{~Hz}, 2 \mathrm{H}$, $\mathrm{CH}_{2} \mathrm{CH}_{2} \mathrm{O}$ ), 1.21 (s, 12H, Bpin), 0.89 (s, 9H, $t \mathrm{Bu}$ ), 0.89 (ddd, $\left.J=3.3,3.3,3.3 \mathrm{~Hz}, 2 \mathrm{H}\right) 0.60$ (ddd, $J=3.3,3.3,3.3 \mathrm{~Hz}, 2 \mathrm{H}), 0.04\left(\mathrm{~s}, 6 \mathrm{H}, \mathrm{Si}\left(\mathrm{CH}_{3}\right)_{2}\right) ;{ }^{13} \mathrm{C} \mathrm{NMR}\left(100 \mathrm{MHz}, \mathrm{CDCl}_{3}\right): \delta 136.2$, 123.9, 83.2, 63.6, 36.6, 26.1, 24.8, 18.5, 14.2, -5.06; IR v 2978 (s), 2929 (s), 1406 (s), 1255 (m), 1141 (s), 1079 (s), 835 (m), 775 (m); HRMS (APCI) calcd. for $\mathrm{C}_{19} \mathrm{H}_{38} \mathrm{BO}_{3} \mathrm{Si}^{+}[\mathrm{M}+\mathrm{H}]^{+}$ 353.2678; found: 353.2670. Carbon attached to boron not observed due to quadrupolar relaxation. See spectra

\section{(E)-4,4,5,5-Tetramethyl-2-(1-(4-phenylbut-1-en-1-yl)cyclopropyl)-1,3,2-dioxaborolane} (4e)

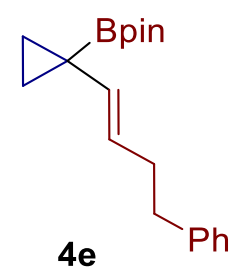

Following general procedure A on a $2.5 \mathrm{mmol}$ scale, using 2,2,6,6-tetramethylpiperidine (385 $\mathrm{mg}, 465 \mu \mathrm{L}, 2.75 \mathrm{mmol}, 1.10$ equiv), $n \mathrm{BuLi}$ (1.75 mL, $2.75 \mathrm{mmol}, 1.60 \mathrm{M}$ in hexanes, 1.10 equiv), cyclopropyl bromide (333 mg, $220 \mu \mathrm{L}, 2.75 \mathrm{mmol}, 1.00$ equiv) and (E)-4,4,5,5tetramethyl-2-(4-phenylbut-1-en-1-yl)-1,3,2-dioxaborolane (645 mg, $2.50 \mathrm{mmol}, 1.00$ equiv). The crude reaction mixture was purified by flash chromatography using 1:10 DCM:pentane to 1:1 DCM:pentane as mobile phase to afford $4 \mathbf{e}(460 \mathrm{mg}, 1.54 \mathrm{mmol}, 62 \%)$ as a colourless liquid. TLC (DCM:pentane, 1:5 v/v): $\mathrm{R}_{\mathrm{f}}=0.31, \mathrm{KMnO}_{4} ;{ }^{1} \mathrm{H} \mathrm{NMR}\left(400 \mathrm{MHz}, \mathrm{CDCl}_{3}\right): \delta 7.29$ $-7.23(\mathrm{~m}, 2 \mathrm{H}, \mathrm{ArH}), 7.20-7.14(\mathrm{~m}, 3 \mathrm{H}, \mathrm{ArH}), 5.55$ (dt, $\left.J=15.5,6.6 \mathrm{~Hz}, 1 \mathrm{H}, \mathrm{CH}=\mathrm{CHCH}_{2}\right)$, $5.42\left(\mathrm{dt}, J=15.5,1.3 \mathrm{~Hz}, 1 \mathrm{H}, \mathrm{CH}=\mathrm{CHCH}_{2}\right), 2.68-2.61\left(\mathrm{~m}, 2 \mathrm{H}, \mathrm{CH}_{2} \mathrm{CH}_{2} \mathrm{Ph}\right), 2.28(\mathrm{dtd}, J=$ 9.0, 6.5, 1.3 Hz, 2H, $\mathrm{CH}_{2} \mathrm{CH}_{2} \mathrm{Ph}$ ), 1.22 (s, 12H, Bpin), 0.89 (ddd, $J=3.4,3.4,3.4 \mathrm{~Hz}, 2 \mathrm{H}$ ), 0.59 (ddd, $J=3.4,3.4,3.4 \mathrm{~Hz}, 2 \mathrm{H}) ;{ }^{13} \mathrm{C} \mathrm{NMR}\left(100 \mathrm{MHz}, \mathrm{CDCl}_{3}\right): \delta 142.5,134.6,128.7,128.3$, 
126.9, 125.7, 83.2, 36.4, 34.8, 24.8, 14.1; IR v 2978 (w), 2028 (w), 1403 (m), 1318 (m), 1136 (s), $967(\mathrm{~m}) 855(\mathrm{~m}), 746(\mathrm{w}), 699(\mathrm{~m})$; HRMS (APCI) calcd. for $\mathrm{C}_{19} \mathrm{H}_{28} \mathrm{BO}_{2}{ }^{+}[\mathrm{M}+\mathrm{H}]^{+}$ 299.2177; found: 299.2174. Carbon attached to boron not observed due to quadrupolar relaxation. See spectra

(E)-2-(1-(2-Cyclopropylvinyl)cyclopropyl)-4,4,5,5-tetramethyl-1,3,2-dioxaborolane (4f)

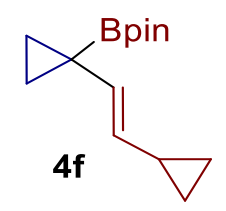

Following general procedure A on a $2.5 \mathrm{mmol}$ scale, using 2,2,6,6-tetramethylpiperidine (385 $\mathrm{mg}, 465 \mu \mathrm{L}, 2.75 \mathrm{mmol}, 1.10$ equiv), $n \mathrm{BuLi}(1.75 \mathrm{~mL}, 2.75 \mathrm{mmol}, 1.60 \mathrm{M}$ in hexanes, 1.10 equiv), cyclopropyl bromide (333 mg, $220 \mu \mathrm{L}, 2.75 \mathrm{mmol}, 1.00$ equiv) and (E)-2-(2cyclopropylvinyl)-4,4,5,5-tetramethyl-1,3,2-dioxaborolane (485 mg, $2.50 \mathrm{mmol}, 1.00$ equiv). The crude reaction mixture was purified by flash chromatography using 1:20 DCM:pentane to 1:1 DCM:pentane as mobile phase to afford $\mathbf{4 f}(286 \mathrm{mg}, 1.22 \mathrm{mmol}, 49 \%)$ as a colourless liquid. TLC (DCM:pentane, 1:5 v/v): $\mathrm{R}_{\mathrm{f}}=0.27, \mathrm{KMnO}_{4} ;{ }^{1} \mathrm{H} \mathrm{NMR}\left(400 \mathrm{MHz}, \mathrm{CDCl}_{3}\right): \delta 5.47$ $(\mathrm{d}, J=15.4 \mathrm{~Hz}, 1 \mathrm{H}, \mathrm{CH}=\mathrm{CHCH}), 5.03(\mathrm{dd}, J=15.4,8.5 \mathrm{~Hz}, 1 \mathrm{H}, \mathrm{CH}=\mathrm{CHCH}), 1.38-1.27$ (m, 1H, $\mathrm{CH}=\mathrm{CHCH}), 1.20$ (s, 12H, Bpin), 0.87 (ddd, $\left.J=3.4,3.4,3.4 \mathrm{~Hz}, 2 \mathrm{H}, \mathrm{BCCH}_{2}\right), 0.62$ (m, $2 \mathrm{H}), 0.58(\mathrm{ddd}, J=3.4,3.4,3.4 \mathrm{~Hz}, 2 \mathrm{H}), 0.31-0.24(\mathrm{~m}, 2 \mathrm{H}) ;{ }^{13} \mathrm{C} \mathrm{NMR}\left(100 \mathrm{MHz}, \mathrm{CDCl}_{3}\right): \delta$ 131.8, 131.3, 83.1, 24.8, 14.1, 13.9, 6.6; IR v 2979 (m), 1407 (m), 1315 (m), 1136 (s), 967 (m), 854 (m), 682 (m); HRMS (APCI) calcd. for $\mathrm{C}_{14} \mathrm{H}_{24} \mathrm{BO}_{2}{ }^{+}[\mathrm{M}+\mathrm{H}]^{+}$235.1864; found: 235.1861 . Carbon attached to boron not observed due to quadrupolar relaxation. See spectra

\section{(E)-4,4,5,5-tetramethyl-2-(1-styrylcyclopropyl)-1,3,2-dioxaborolane (4g)}

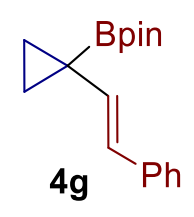

Following general procedure A on a $2.5 \mathrm{mmol}$ scale, using 2,2,6,6-tetramethylpiperidine (385 $\mathrm{mg}, 465 \mu \mathrm{L}, 2.75 \mathrm{mmol}, 1.10$ equiv), $n \mathrm{BuLi}(1.75 \mathrm{~mL}, 2.75 \mathrm{mmol}, 1.60 \mathrm{M}$ in hexanes, 1.10 equiv), cyclopropyl bromide (333 $\mathrm{mg}, 220 \mu \mathrm{L}, 2.75 \mathrm{mmol}, 1.00$ equiv) and (E)-4,4,5,5tetramethyl-2-styryl-1,3,2-dioxaborolane ( $575 \mathrm{mg}, 2.50 \mathrm{mmol}, 1.00$ equiv). The crude reaction mixture was purified by flash chromatography using 1:4 DCM:pentane as mobile phase to 
afford $4 \mathbf{f}$ (377 mg, $1.40 \mathrm{mmol}, 56 \%)$ as a white solid. TLC (DCM:pentane, 1:3 v/v): $\mathrm{R}_{\mathrm{f}}=0.24$, $\mathrm{KMnO}_{4}$; M.p. $45-50{ }^{\circ} \mathrm{C} ;{ }^{1} \mathrm{H}$ NMR $\left(400 \mathrm{MHz}, \mathrm{CDCl}_{3}\right): \delta 7.34-7.29(\mathrm{~m}, 2 \mathrm{H}, \mathrm{ArH}), 7.29-$ $7.22(\mathrm{~m}, 2 \mathrm{H}, \mathrm{ArH}), 7.14(\mathrm{tt}, J=7.2,1.3 \mathrm{~Hz}, 1 \mathrm{H}, \mathrm{Ar} H), 6.50$ (d, $J=16.0 \mathrm{~Hz}, 1 \mathrm{H}, \mathrm{CH}=\mathrm{CHPh})$, $6.16(\mathrm{~d}, J=16.0 \mathrm{~Hz}, 1 \mathrm{H}, \mathrm{CH}=\mathrm{CHPh}), 1.25$ (s, 12H, Bpin), 1.06 (ddd, $J=3.5,3.5,3.5 \mathrm{~Hz}, 2 \mathrm{H})$, $0.80(\mathrm{ddd}, J=3.5,3.5,3.5 \mathrm{~Hz}, 2 \mathrm{H}) ;{ }^{13} \mathrm{C} \mathrm{NMR}\left(100 \mathrm{MHz}, \mathrm{CDCl}_{3}\right): \delta 138.5,135.4,128.5,127.1$, 126.5, 125.9, 83.4, 24.9, 14.9; IR v 2979 (m), 2932 (w), 1645 (w), 1402 (m), $1321(\mathrm{~m}), 1135$ (s), 967 (m), 854 (m), $744(\mathrm{~m}), 693$ (m); HRMS (APCI) calcd. for $\mathrm{C}_{17} \mathrm{H}_{23} \mathrm{BO}_{2}{ }^{+}[\mathrm{M}]^{+}$270.1786; found: 270.1784 . Carbon attached to boron not observed due to quadrupolar relaxation. See spectra

\section{4,4,5,5-Tetramethyl-2-(1-(prop-1-en-2-yl)cyclopropyl)-1,3,2-dioxaborolane (4h)}

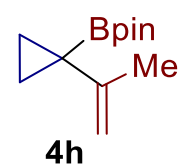

Following general procedure A, using 2,2,6,6-tetramethylpiperidine (770 mg, $930 \mu \mathrm{L}, 5.50$ mmol, 1.10 equiv), ${ }^{n} \mathrm{BuLi}(3.44 \mathrm{~mL}, 5.50 \mathrm{mmol}, 1.60 \mathrm{M}$ in hexanes, 1.10 equiv), cyclopropyl bromide (665 m, $440 \mu \mathrm{L}, 5.50 \mathrm{mmol}, 1.00$ equiv) and 4,4,5,5-tetramethyl-2-(prop-1-en-2-yl)1,3,2-dioxaborolane ( $840 \mathrm{mg}, 5.00 \mathrm{mmol}, 1.00$ equiv). The crude reaction mixture was purified by flash chromatography using DCM:pentane 1:10 as mobile phase to afford $\mathbf{4 h}$ ( $852 \mathrm{mg}, 4.09$ mmol, $82 \%$ ) as a white solid. Mp: $39.5-44.3{ }^{\circ} \mathrm{C}$; TLC (DCM:pentane, 1:5 v/v): $\mathrm{R}_{\mathrm{f}}=0.24$, $\mathrm{KMnO}_{4} ;{ }^{1} \mathrm{H}$ NMR $\left(400 \mathrm{MHz}, \mathrm{CDCl}_{3}\right): \delta 4.73\left(\mathrm{p}, J=1.4 \mathrm{~Hz}, 1 \mathrm{H}, \mathrm{C}=\mathrm{CH}^{a} \mathrm{H}^{\mathrm{b}}\right), 4.68-4.69(\mathrm{~m}$, $\left.1 \mathrm{H}, \mathrm{C}=\mathrm{CH}^{\mathrm{a}} H^{b}\right), 1.83-1.80\left(\mathrm{~m}, 3 \mathrm{H}, \mathrm{CH}_{3} \mathrm{C}=\mathrm{CH}_{2}\right), 1.21(\mathrm{~s}, 12 \mathrm{H}, \mathrm{Bpin}), 0.86-0.77(\mathrm{~m}, 2 \mathrm{H})$, $0.69-0.55(\mathrm{~m}, 2 \mathrm{H}) ;{ }^{13} \mathrm{C} \mathrm{NMR}\left(100 \mathrm{MHz}, \mathrm{CDCl}_{3}\right): \delta 148.6,109.7,83.1,24.55,23.0,11.7$; IR v $2980(\mathrm{~s}), 1421(\mathrm{~m}), 1368(\mathrm{~s}), 1325$ (s), 1155 (m), 1127 (s), 1023 (m), 964 (m), 885 (s); HRMS (APCI) calcd. for $\mathrm{C}_{12} \mathrm{H}_{22} \mathrm{BO}_{2}{ }^{+}[\mathrm{M}+\mathrm{H}]^{+}$209.1707; found 209.1703. Carbon attached to boron not observed due to quadrupolar relaxation. See spectra

\section{2-(1-(Cyclohex-1-en-1-yl)cyclopropyl)-4,4,5,5-tetramethyl-1,3,2-dioxaborolane (4i)}

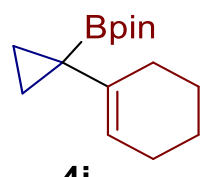

$4 \mathbf{i}$ 
Following general procedure A, using 2,2,6,6-tetramethylpiperidine (770 mg, $930 \mu \mathrm{L}, 5.50$ mmol, 1.10 equiv), ${ }^{n} \mathrm{BuLi}(3.44 \mathrm{~mL}, 5.50 \mathrm{mmol}, 1.60 \mathrm{M}$ in hexanes, 1.10 equiv), cyclopropyl bromide (665 mg, $440 \mu \mathrm{L}, 5.50 \mathrm{mmol}, 1.00$ equiv) and 4-(4,4,5,5-tetramethyl-1,3,2dioxaborolan-2-yl)cyclohex-3-en-1-ylium (770 mg, $5.00 \mathrm{mmol}, 1.00$ equiv). The crude reaction mixture was purified by flash chromatography using DCM:pentane 1:10 as mobile phase to afford $4 \mathbf{i}$ (882 $\mathrm{mg}, 3.55 \mathrm{mmol}, 71 \%$ ) as a colorless liquid. TLC (DCM:pentane, 1:5 $\mathrm{v} / \mathrm{v}): \mathrm{R}_{\mathrm{f}}=0.28, \mathrm{KMnO}_{4} ;{ }^{1} \mathrm{H} \mathrm{NMR}\left(400 \mathrm{MHz}, \mathrm{CDCl}_{3}\right): \delta 5.37(\mathrm{tt}, J=3.7,1.7 \mathrm{~Hz}, 1 \mathrm{H}$, $\left.\mathrm{C}=\mathrm{CHCH}_{2}\right), 2.07-2.02\left(\mathrm{~m}, 2 \mathrm{H}, \mathrm{CH}_{2}\right), 1.99-1.93\left(\mathrm{~m}, 2 \mathrm{H}, \mathrm{CH}_{2}\right), 1.63-1.56\left(\mathrm{~m}, 2 \mathrm{H}, \mathrm{CH}_{2}\right)$, $1.55-1.50\left(\mathrm{~m}, 2 \mathrm{H}, \mathrm{CH}_{2}\right), 1.20$ (s, 12H, Bpin), $0.78-0.69(\mathrm{~m}, 2 \mathrm{H}), 0.61-0.51(\mathrm{~m}, 2 \mathrm{H}) ;{ }^{13} \mathrm{C}$ NMR (100 MHz, $\left.\mathrm{CDCl}_{3}\right): \delta$ 140.4, 120.2, 82.9, 29.1, 25.2, 24.6, 23.3, 22.7, 11.0; IR v 2977 (m), 2925 (m), 2836 (w), 1426 (m), 1387 (s), 1312 (m), 1579 (w), 1165 (m), 1141 (s), 921 (w), 854 (m); HRMS (APCI) calcd. for $\mathrm{C}_{15} \mathrm{H}_{26} \mathrm{BO}_{2}{ }^{+}[\mathrm{M}+\mathrm{H}]^{+}$249.2020; found 249.2010. Carbon attached to boron not observed due to quadrupolar relaxation. See spectra

\section{$N, N$-Dimethyl-2-((1s,2r)-2-phenyl-2-(4,4,5,5-tetramethyl-1,3,2-dioxaborolan-2- yl)cyclobutyl)ethan-1-amine hydrochloride (1a)}

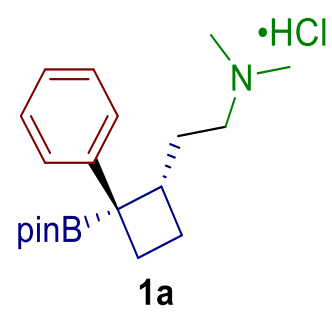

Following general procedure B, using 4,4,5,5-tetramethyl-2-(1-vinylcyclopropyl)-1,3,2dioxaborolane (4a) (55.3 mg, $0.300 \mathrm{mmol}, 1.00$ equiv), PhLi (206 $\mu \mathrm{L}, 0.390 \mathrm{mmol}, 1.9 \mathrm{M}$ in in dibutyl ether, 1.30 equiv), and Eschenmoser's salt (5) (111 mg, $0.600 \mathrm{mmol}, 2.00$ equiv). The crude material was then dissolved in $\mathrm{Et}_{2} \mathrm{O}$ (ca. $\left.0.6 \mathrm{~mL}\right)$ and then $2 \mathrm{~N} \mathrm{HCl}(165 \mu \mathrm{L}, 0.330$ mmol, 1.10 equiv) in $\mathrm{Et}_{2} \mathrm{O}$ was added. A white precipitate was formed, which was collected via a suction filtration (pentane was used for transfer of the compound) and then thoroughly dried under high vacuum to afford 1a (103 mg, $0.282 \mathrm{mmol}, 94 \%,>20: 1 \mathrm{dr}$ ) as an off-white solid. Mp: $218.2-220.5{ }^{\circ} \mathrm{C} ;{ }^{1} \mathrm{H}$ NMR (400 MHz, $\left.\mathrm{CDCl}_{3}\right): \delta 12.56\left(\mathrm{~s}, 1 \mathrm{H},\left(\mathrm{CH}_{3}\right)_{2} \mathrm{~N} . \mathrm{HCl}\right), 7.36$ - $7.22(\mathrm{~m}, 2 \mathrm{H}, \mathrm{ArH}), 7.18-7.00(\mathrm{~m}, 3 \mathrm{H}, \mathrm{ArH}), 3.09-2.97\left(\mathrm{~m}, 2 \mathrm{H}, \mathrm{CH}_{2}\left(\mathrm{CH}_{3}\right)_{2} \mathrm{~N}\right), 2.82$ (br s, $\left.6 \mathrm{H},\left(\mathrm{CH}_{3}\right)_{2} \mathrm{~N} . \mathrm{HCl}\right), 2.60-2.48(\mathrm{~m}, 2 \mathrm{H}), 2.30-2.22(\mathrm{~m}, 2 \mathrm{H}), 2.11-2.01(\mathrm{~m}, 2 \mathrm{H}), 1.77-1.68$ $(\mathrm{m}, 1 \mathrm{H}), 1.24\left(\mathrm{~s}, 6 \mathrm{H}, \mathrm{OC}\left(\mathrm{CH}_{3}\right)_{2}\right), 1.22\left(\mathrm{~s}, 6 \mathrm{H}, \mathrm{OC}\left(\mathrm{CH}_{3}\right)_{2}\right) ;{ }^{13} \mathrm{C} \mathrm{NMR}\left(100 \mathrm{MHz}, \mathrm{CDCl}_{3}\right): \delta$ $147.7,128.3,125.3,125.1,83.8,56.3,43.2,42.2,42.2,29.7,29.5,25.3,24.8,24.7$; IR v 3419 
(m), 2974 (m), 2937 (w), 1479 (w), 1444 (w), 1342 (s), 1312 (s), 1141 (s), 1105 (m), 954 (w); HRMS (ESI) calcd. for $\mathrm{C}_{20} \mathrm{H}_{33} \mathrm{NO}_{2} \mathrm{~B}^{+}[\mathrm{M}+\mathrm{H}]^{+}$330.2599; found 330.2589. Carbon attached to boron not observed due to quadrupolar relaxation. See spectra

\section{2-((1s,2r)-2-(4-Methoxyphenyl)-2-(4,4,5,5-tetramethyl-1,3,2-dioxaborolan-2-} yl)cyclobutyl)- $N, N$-dimethylethan-1-amine hydrochloride (1b)

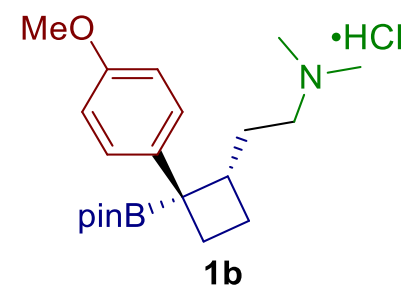

Following general procedure $\mathbf{C}$, using 1-bromo-4-methoxybenzene $(79.0 \mathrm{mg}, 0.420 \mathrm{mmol}$, 1.40 equiv), $n \mathrm{BuLi}(245 \mu \mathrm{L}, 0.390 \quad \mathrm{mmol}, 1.30$ equiv), 4,4,5,5-tetramethyl-2-(1vinylcyclopropyl)-1,3,2-dioxaborolane (4a) (55.3 mg, $0.300 \mathrm{mmol}, 1.00$ equiv), and Eschenmoser's salt (5) $(111 \mathrm{mg}, 0.600 \mathrm{mmol}, 2.00$ equiv). The crude material was then dissolved in $\mathrm{Et}_{2} \mathrm{O}$ (ca. $0.6 \mathrm{~mL}$ ) and then $2 \mathrm{~N} \mathrm{HCl}\left(165 \mu \mathrm{L}, 0.330 \mathrm{mmol}, 1.10\right.$ equiv) in $\mathrm{Et}_{2} \mathrm{O}$ was added. A white precipitate was formed, which was collected via a suction filtration (pentane was used for transfer of the compound) and then thoroughly dried under high vacuum to afford $1 \mathbf{b}$ (96.0 mg, $0.243 \mathrm{mmol}, 81 \%$, >20:1 dr) as an off-white solid. Mp: $176.5-182.4{ }^{\circ} \mathrm{C}$ (Dec.); ${ }^{1} \mathrm{H}$ NMR (400 MHz, $\left.\mathrm{CDCl}_{3}\right): \delta 12.47\left(\mathrm{~s}, 1 \mathrm{H},\left(\mathrm{CH}_{3}\right)_{2} \mathrm{~N} . H \mathrm{Cl}\right), 6.99(\mathrm{~d}, J=8.6 \mathrm{~Hz}, 2 \mathrm{H}, \mathrm{ArH})$, $6.80(\mathrm{~d}, J=8.6 \mathrm{~Hz}, 2 \mathrm{H}, \mathrm{ArH}), 3.77\left(\mathrm{~s}, 3 \mathrm{H}, \mathrm{OCH}_{3}\right), 3.08-2.89\left(\mathrm{~m}, 2 \mathrm{H}, \mathrm{CH}_{2}\left(\mathrm{CH}_{3}\right)_{2} \mathrm{~N}\right), 2.78(\mathrm{t}$, $\left.J=4.4 \mathrm{~Hz}, 6 \mathrm{H},\left(\mathrm{CH}_{3}\right)_{2} \mathrm{~N} . H \mathrm{HCl}\right), 2.53-2.38(\mathrm{~m}, 2 \mathrm{H}), 2.28-2.13(\mathrm{~m}, 2 \mathrm{H}), 2.08-1.91(\mathrm{~m}, 2 \mathrm{H})$, $1.71-1.61(\mathrm{~m}, 1 \mathrm{H}), 1.22\left(\mathrm{~s}, 6 \mathrm{H}, \mathrm{OC}\left(\mathrm{CH}_{3}\right)_{2}\right), 1.20\left(\mathrm{~s}, 6 \mathrm{H}, \mathrm{OC}\left(\mathrm{CH}_{3}\right)_{2}\right) ;{ }^{13} \mathrm{C} \mathrm{NMR}(100 \mathrm{MHz}$, $\left.\mathrm{CDCl}_{3}\right): \delta 157.2,139.6,126.4,113.7,83.7,56.3,55.2,43.2,42.5,42.2,29.6,29.5,25.2,24.8$, 24.7; IR v 3411 (s), 2973 (s), 1508 (w), 1509 (s), 1469 (m), 1314 (m), 1298 (m), 1244 (s), 1142 (s), 1035 (m); HRMS (ESI) calcd. for $\mathrm{C}_{21} \mathrm{H}_{35} \mathrm{NO}_{3} \mathrm{~B}^{+}[\mathrm{M}+\mathrm{H}]^{+}$360.2705; found 360.2695. Carbon attached to boron not observed due to quadrupolar relaxation. 
2-((1s,2r)-2-(3,5-Bis(trifluoromethyl)phenyl)-2-(4,4,5,5-tetramethyl-1,3,2-dioxaborolan2-yl)cyclobutyl)- $N, N$-dimethylethan-1-amine hydrochloride (1c)

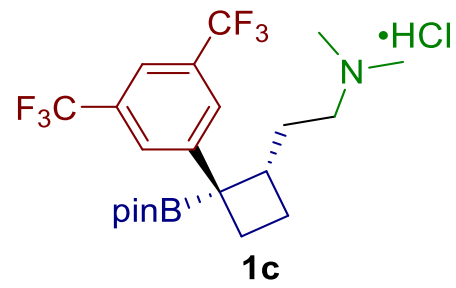

Following general procedure C, using 1-bromo-3,5-bis(trifluoromethyl)benzene (123 mg, 0.420 mmol, 1.40 equiv), $n \mathrm{BuLi}(245 \mu \mathrm{L}, 0.390 \mathrm{mmol}, 1.30$ equiv), 4,4,5,5-tetramethyl-2-(1vinylcyclopropyl)-1,3,2-dioxaborolane (4a) $(55.3 \mathrm{mg}, 0.300 \mathrm{mmol}, 1.00$ equiv), and Eschenmoser's salt (5) $(111 \mathrm{mg}, 0.600 \mathrm{mmol}, 2.00$ equiv). The crude material was then dissolved in pentane (ca. $0.6 \mathrm{~mL}$ ) and then $2 \mathrm{~N} \mathrm{HCl}\left(165 \mu \mathrm{L}, 0.330 \mathrm{mmol}, 1.10\right.$ equiv) in $\mathrm{Et}_{2} \mathrm{O}$ was added. A white precipitate was formed, which was collected via a suction filtration (pentane was used for transfer of the compound) and then thoroughly dried under high vacuum to afford 1c (137 mg, $0.273 \mathrm{mmol}, 91 \%$, > 20:1 dr) as an off-white solid. Mp: $87.5-91.5^{\circ} \mathrm{C}$; ${ }^{1} \mathrm{H}$ NMR (400 MHz, $\left.\mathrm{CDCl}_{3}\right): \delta 12.69\left(\mathrm{~s}, 1 \mathrm{H},\left(\mathrm{CH}_{3}\right)_{2} \mathrm{~N} . \mathrm{HCl}\right), 7.64(\mathrm{~s}, 1 \mathrm{H}, \mathrm{ArH}), 7.49$ (s, 2H, $\mathrm{ArH}), 3.02-2.91\left(\mathrm{~m}, 2 \mathrm{H}, \mathrm{CH}_{2}\left(\mathrm{CH}_{3}\right)_{2} \mathrm{~N}\right), 2.83-2.79\left(\mathrm{~m}, 6 \mathrm{H},\left(\mathrm{CH}_{3}\right)_{2} \mathrm{~N} . H \mathrm{Cl}\right), 2.59(\mathrm{t}, J=8.7$ $\mathrm{Hz}, 1 \mathrm{H}), 2.55-2.48(\mathrm{~m}, 1 \mathrm{H}), 2.35-2.21(\mathrm{~m} 2 \mathrm{H}), 2.15-2.02(\mathrm{~m}, 2 \mathrm{H}), 1.93-1.71(\mathrm{~m}, 1 \mathrm{H})$, $1.24\left(\mathrm{~s}, 6 \mathrm{H}, \mathrm{OC}\left(\mathrm{CH}_{3}\right)_{2}\right), 1.22\left(\mathrm{~s}, 6 \mathrm{H}, \mathrm{OC}\left(\mathrm{CH}_{3}\right)_{2}\right) ;{ }^{13} \mathrm{C} \mathrm{NMR}\left(100 \mathrm{MHz}, \mathrm{CDCl}_{3}\right): \delta 150.4,131.4$ $(\mathrm{q}, J=32.9 \mathrm{~Hz}), 125.6(\mathrm{~m}, \mathrm{q}, J=3.1 \mathrm{~Hz}), 123.4(\mathrm{q}, J=272.6 \mathrm{~Hz}), 119.2$ (sept, $J=3.7 \mathrm{~Hz})$,

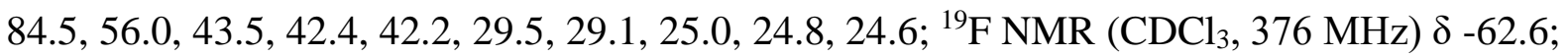
IR v 3414 (w), 2978 (w), 1458 (w), 1373 (s), 1275 (s), 1125 (s), 952 (w), 848 (m); HRMS (ESI) calcd. for $\mathrm{C}_{22} \mathrm{H}_{31} \mathrm{NO}_{2} \mathrm{BF}_{6}{ }^{+}[\mathrm{M}+\mathrm{H}]^{+}$466.2347; found 466.2336. Carbon attached to boron not observed due to quadrupolar relaxation.

2-((1s,2r)-2-(2-Fluorophenyl)-2-(4,4,5,5-tetramethyl-1,3,2-dioxaborolan-2-yl)cyclobutyl)$\mathrm{N}, \mathrm{N}$-dimethylethan-1-amine hydrochloride (1d)

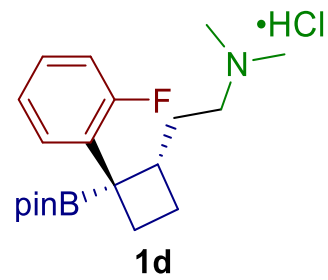


Following general procedure $\mathbf{C}$, using 1-bromo-2-fluorobenzene (73.5 mg, $0.420 \mathrm{mmol}, 1.40$ equiv), $n \mathrm{BuLi}$ (245 $\mu \mathrm{L}, 0.390 \mathrm{mmol}, 1.30$ equiv), 4,4,5,5-tetramethyl-2-(1-vinylcyclopropyl)1,3,2-dioxaborolane (4a) (55.3 mg, 0.300 mmol, 1.00 equiv), and Eschenmoser's salt (5) (111 $\mathrm{mg}, 0.600 \mathrm{mmol}, 2.00$ equiv). The crude material was then dissolved in $\mathrm{Et}_{2} \mathrm{O}$ (ca. $0.6 \mathrm{~mL}$ ) and then $2 \mathrm{~N} \mathrm{HCl}(165 \mu \mathrm{L}, 0.330 \mathrm{mmol}, 1.10$ equiv $)$ in $\mathrm{Et}_{2} \mathrm{O}$ was added. A white precipitate was formed, which was collected via a suction filtration (pentane was used for transfer of the compound) and then thoroughly dried under high vacuum to afford $1 \mathbf{1 d}(106 \mathrm{mg}, 0.276 \mathrm{mmol}$, $92 \%,>20: 1 \mathrm{dr}$ ) as an off-white solid. Mp: $204.5-206.3{ }^{\circ} \mathrm{C} ;{ }^{1} \mathrm{H}$ NMR $\left(400 \mathrm{MHz}, \mathrm{CDCl}_{3}\right): \delta$ $12.52\left(\mathrm{~s}, 1 \mathrm{H},\left(\mathrm{CH}_{3}\right)_{2} \mathrm{~N} . H \mathrm{Cl}\right), 7.16-7.01(\mathrm{~m}, 3 \mathrm{H}, \mathrm{ArH}), 6.99-6.88(\mathrm{~m}, 1 \mathrm{H}, \mathrm{ArH}), 3.02-2.91$ $\left(\mathrm{m}, 2 \mathrm{H}, \mathrm{CH}_{2}\left(\mathrm{CH}_{3}\right)_{2} \mathrm{~N}\right), 2.79-2.76\left(\mathrm{~m}, 6 \mathrm{H},\left(\mathrm{CH}_{3}\right)_{2} \mathrm{~N} . \mathrm{HCl}\right), 2.61-2.52(\mathrm{~m}, 1 \mathrm{H}), 2.48(\mathrm{t}, J=8.7$ $\mathrm{Hz}, 1 \mathrm{H}), 2.29-2.15(\mathrm{~m} \mathrm{2H}), 2.11-1.95(\mathrm{~m}, 2 \mathrm{H}), 1.93-1.85(\mathrm{~m}, 1 \mathrm{H}), 1.24\left(\mathrm{~s}, 6 \mathrm{H}, \mathrm{OC}\left(\mathrm{CH}_{3}\right)_{2}\right)$, $1.23\left(\mathrm{~s}, 6 \mathrm{H}, \mathrm{OC}\left(\mathrm{CH}_{3}\right)_{2}\right) ;{ }^{13} \mathrm{C} \mathrm{NMR}\left(100 \mathrm{MHz}, \mathrm{CDCl}_{3}\right): \delta 160.1(\mathrm{~d}, J=242.9 \mathrm{~Hz}), 134.8(\mathrm{~d}, J=$ $16.0 \mathrm{~Hz}), 126.7$ (d, $J=8.2 \mathrm{~Hz}), 126.6(\mathrm{~d}, J=5.5 \mathrm{~Hz}), 124.0$ (d, $J=3.2 \mathrm{~Hz}), 115.0$ (d, $J=22.3$ Hz), 83.8, 56.0, 43.0, 42.2, 41.0, 28.6 (d, $J=2.8 \mathrm{~Hz}), 28.6$ ((d, $J=1.3 \mathrm{~Hz})$,), 24.9, 24.9, 24.6; ${ }^{19} \mathrm{~F} \mathrm{NMR}\left(\mathrm{CDCl}_{3}, 376 \mathrm{MHz}\right) \delta-114.45$ (dd, $\left.J=11.0,7.0 \mathrm{~Hz}\right)$; IR $v 3399$ (m), 2975 (m), 2939 (m), 1484 (s), 1344 (s), 1371 (s), 1317 (s), 1141 (s), 1108 (m), 954 (m), 846 (m); HRMS (ESI) calcd. for $\mathrm{C}_{20} \mathrm{H}_{32} \mathrm{NO}_{2} \mathrm{BF}^{+}[\mathrm{M}+\mathrm{H}]^{+} 348.2505$; found 348.2499. Carbon attached to boron not observed due to quadrupolar relaxation. See spectra

\section{2-((1s,2r)-2-(4-Chlorophenyl)-2-(4,4,5,5-tetramethyl-1,3,2-dioxaborolan-2-}

\section{yl)cyclobutyl)-N,N-dimethylethan-1-amine hydrochloride (1e)}

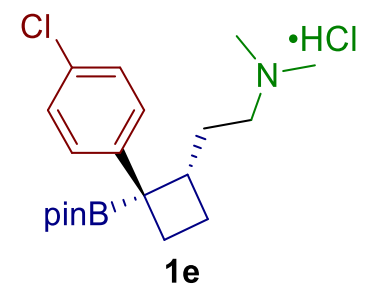

Following general procedure C, using 1-chloro-4-iodobenzene (101 mg, $0.420 \mathrm{mmol}, 1.40$ equiv), $n \mathrm{BuLi}$ (245 $\mu \mathrm{L}, 0.390 \mathrm{mmol}, 1.30$ equiv), 4,4,5,5-tetramethyl-2-(1-vinylcyclopropyl)1,3,2-dioxaborolane (4a) (55.3 mg, 0.300 mmol, 1.00 equiv), and Eschenmoser's salt (5) (111 $\mathrm{mg}, 0.600 \mathrm{mmol}, 2.00$ equiv). The crude material was then dissolved in $\mathrm{Et}_{2} \mathrm{O}$ (ca. $0.6 \mathrm{~mL}$ ) and then $2 \mathrm{~N} \mathrm{HCl}(165 \mu \mathrm{L}, 0.330 \mathrm{mmol}, 1.10$ equiv $)$ in $\mathrm{Et}_{2} \mathrm{O}$ was added. A white precipitate was formed, which was collected via a suction filtration (pentane was used for transfer of the compound) and then thoroughly dried under high vacuum to afford 1e (101 $\mathrm{mg}, 0.253 \mathrm{mmol}$, 
$84 \%,>20: 1 \mathrm{dr}$ ) as an off-white solid. Mp: $208.5-211.4{ }^{\circ} \mathrm{C} ;{ }^{1} \mathrm{H}$ NMR $\left(400 \mathrm{MHz}, \mathrm{CDCl}_{3}\right): \delta$ $12.5\left(\mathrm{~s}, 1 \mathrm{H},\left(\mathrm{CH}_{3}\right)_{2} \mathrm{~N} . H \mathrm{Cl}\right), 7.21(\mathrm{~d}, J=8.5 \mathrm{~Hz}, 2 \mathrm{H}, \operatorname{Ar} H), 6.99(\mathrm{~d}, J=8.5 \mathrm{~Hz}, 2 \mathrm{H}, \operatorname{Ar} H), 3.05$ $-2.85\left(\mathrm{~m}, 2 \mathrm{H}, \mathrm{CH}_{2}\left(\mathrm{CH}_{3}\right)_{2} \mathrm{~N}\right), 2.81-2.77\left(\mathrm{~m}, 6 \mathrm{H},\left(\mathrm{CH}_{3}\right)_{2} \mathrm{~N} . H \mathrm{Cl}\right), 2.57-2.40(\mathrm{~m}, 2 \mathrm{H}), 2.31-$ $2.13(\mathrm{~m}, 2 \mathrm{H}), 2.10-1.90(\mathrm{~m}, 2 \mathrm{H}), 1.75-1.64(\mathrm{~m}, 1 \mathrm{H}), 1.21\left(\mathrm{~s}, 6 \mathrm{H}, \mathrm{OC}\left(\mathrm{CH}_{3}\right)_{2}\right), 1.19(\mathrm{~s}, 6 \mathrm{H}$, $\left.\mathrm{OC}\left(\mathrm{CH}_{3}\right)_{2}\right) ;{ }^{13} \mathrm{C} \mathrm{NMR}\left(100 \mathrm{MHz}, \mathrm{CDCl}_{3}\right): \delta 146.3,130.7,128.3,126.7,83.9,56.2,43.1,42.5$, 42.4, 29.6, 29.3, 25.2, 24.8, 24.7; IR v 3415 (m), 2974 (m), 1489 (m), 1371 (m), 1141 (s), 1089 (w), 954 (w); HRMS (ESI) calcd. for $\mathrm{C}_{20} \mathrm{H}_{32} \mathrm{NO}_{2} \mathrm{BCl}^{+}[\mathrm{M}+\mathrm{H}]^{+}$364.2209; found 364.2202. Carbon attached to boron not observed due to quadrupolar relaxation. See spectra

\section{2-((1s,2r)-2-(3-Bromo-5-methoxyphenyl)-2-(4,4,5,5-tetramethyl-1,3,2-dioxaborolan-2-} yl)cyclobutyl)- $N, N$-dimethylethan-1-amine hydrochloride (1f)

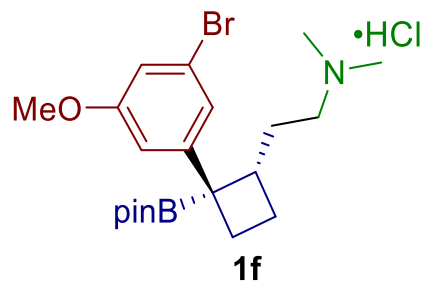

Following general procedure $\mathbf{C}$, using 1,3-dibromo-5-methoxybenzene (112 mg, $0.420 \mathrm{mmol}$, 1.40 equiv), $n \operatorname{BuLi}(245 \mu \mathrm{L}, 0.390 \quad$ mmol, 1.30 equiv), 4,4,5,5-tetramethyl-2-(1vinylcyclopropyl)-1,3,2-dioxaborolane (4a) (55.3 $\mathrm{mg}, 0.300 \mathrm{mmol}, 1.00$ equiv), and Eschenmoser's salt (5) $(111 \mathrm{mg}, 0.600 \mathrm{mmol}, 2.00$ equiv). The crude material was then dissolved in $\mathrm{Et}_{2} \mathrm{O}$ (ca. $\left.0.6 \mathrm{~mL}\right)$ and then $2 \mathrm{~N} \mathrm{HCl}\left(165 \mu \mathrm{L}, 0.330 \mathrm{mmol}, 1.10\right.$ equiv) in $\mathrm{Et}_{2} \mathrm{O}$ was added. A white precipitate was formed, which was collected via a suction filtration (pentane was used for transfer of the compound) and then thoroughly dried under high vacuum to afford 1f (114 mg, $0.240 \mathrm{mmol}, 80 \%$, > 20:1 dr) as an off-white solid. Mp: $194.3-197.5{ }^{\circ} \mathrm{C} ;{ }^{1} \mathrm{H}$ NMR $\left(400 \mathrm{MHz}, \mathrm{CDCl}_{3}\right): \delta 12.61\left(\mathrm{~s}, 1 \mathrm{H},\left(\mathrm{CH}_{3}\right)_{2} \mathrm{~N} . \mathrm{HCl}\right), 6.81(\mathrm{~s}, 1 \mathrm{H}, \mathrm{ArH}), 6.78(\mathrm{~s}, 1 \mathrm{H}, \operatorname{ArH}), 6.53$ (s, 1H, $\mathrm{ArH}$ ), $3.77\left(\mathrm{~s}, 3 \mathrm{H}, \mathrm{ArOCH}_{3}\right), 2.97\left(\mathrm{t}, J=8.2 \mathrm{~Hz}, 2 \mathrm{H}, \mathrm{CH}_{2}\left(\mathrm{CH}_{3}\right)_{2} \mathrm{~N}\right), 2.79$ (br s, 6H, $\left.\left(\mathrm{CH}_{3}\right)_{2} \mathrm{~N} . \mathrm{HCl}\right), 2.55-2.41(\mathrm{~m}, 2 \mathrm{H}), 2.29-2.14(\mathrm{~m}, 2 \mathrm{H}), 2.08-1.95(\mathrm{~m}, 2 \mathrm{H}), 1.76-1.66(\mathrm{~m}$, 1H), $1.24\left(\mathrm{~s}, 6 \mathrm{H}, \mathrm{OC}\left(\mathrm{CH}_{3}\right)_{2}\right), 1.22\left(\mathrm{~s}, 6 \mathrm{H}, \mathrm{OC}\left(\mathrm{CH}_{3}\right)_{2}\right) ;{ }^{13} \mathrm{C} \mathrm{NMR}\left(100 \mathrm{MHz}, \mathrm{CDCl}_{3}\right): \delta 160.2$, 151.1, 122.7, 121.0, 113.5, 110.9, 84.0, 56.3, 55.5, 43.4, 42.2, 42.1, 29.6, 29.4, 25.1, 24.8, 24.7; IR v 3410 (m), 2973 (m), 1594 (m), 1564 (m), 1418 (w), 1315 (s), 1141 (s), 1058 (w), 963 (w); HRMS (ESI) calcd. for $\mathrm{C}_{21} \mathrm{H}_{34} \mathrm{BBrNO}_{3}{ }^{+}[\mathrm{M}+\mathrm{H}]^{+}$438.1813; found 438.1817. Carbon attached to boron not observed due to quadrupolar relaxation. See spectra 
2-((1s,2r)-2-([1,1'-Biphenyl]-4-yl)-2-(4,4,5,5-tetramethyl-1,3,2-dioxaborolan-2yl)cyclobutyl)- $N, N$-dimethylethan-1-amine hydrochloride (1g)

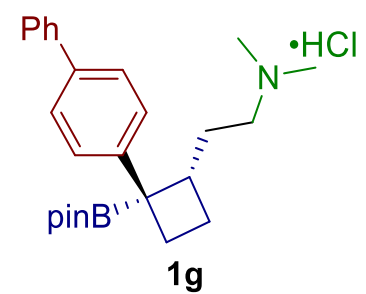

Following general procedure C, using 4-bromo-1,1'-biphenyl (98.0 mg, $0.420 \mathrm{mmol}, 1.40$ equiv), $n \mathrm{BuLi}$ (245 $\mu \mathrm{L}, 0.390 \mathrm{mmol}, 1.30$ equiv), 4,4,5,5-tetramethyl-2-(1-vinylcyclopropyl)1,3,2-dioxaborolane (4a) (55.3 mg, 0.300 mmol, 1.00 equiv), and Eschenmoser's salt (5) (111 $\mathrm{mg}, 0.600 \mathrm{mmol}, 2.00$ equiv). The crude material was then dissolved in $\mathrm{Et}_{2} \mathrm{O}$ (ca. $0.6 \mathrm{~mL}$ ) and then $2 \mathrm{~N} \mathrm{HCl}\left(165 \mu \mathrm{L}, 0.330 \mathrm{mmol}, 1.10\right.$ equiv) in $\mathrm{Et}_{2} \mathrm{O}$ was added. A white precipitate was formed, which was collected via a suction filtration (pentane was used for transfer of the compound) and then thoroughly dried under high vacuum to afford $1 \mathrm{~g}$ (127 $\mathrm{mg}, 0.288 \mathrm{mmol}$, $96 \%,>20: 1 \mathrm{dr}$ ) as an off-white solid. Mp: $210.5-213.0{ }^{\circ} \mathrm{C} ;{ }^{1} \mathrm{H}$ NMR $\left(400 \mathrm{MHz}, \mathrm{CDCl}_{3}\right): \delta$ $12.54\left(\mathrm{~s}, 1 \mathrm{H},\left(\mathrm{CH}_{3}\right)_{2} \mathrm{~N} . H \mathrm{Cl}\right), 7.59-7.55(\mathrm{~m}, 2 \mathrm{H}, \mathrm{ArH}), 7.50(\mathrm{~d}, J=8.3 \mathrm{~Hz}, 2 \mathrm{H}, \mathrm{ArH}), 7.42$ (t, $J=7.7 \mathrm{~Hz}, 2 \mathrm{H}, \mathrm{ArH}), 7.35-7.29(\mathrm{~m}, 1 \mathrm{H}, \mathrm{ArH}), 7.14(\mathrm{~d}, J=8.3 \mathrm{~Hz}, 2 \mathrm{H}, \operatorname{ArH}), 3.09-2.93$ (m, $\left.2 \mathrm{H}, \mathrm{CH}_{2}\left(\mathrm{CH}_{3}\right)_{2} \mathrm{~N}\right), 2.81-2.79\left(\mathrm{~m}, 6 \mathrm{H},\left(\mathrm{CH}_{3}\right)_{2} \mathrm{~N} . H \mathrm{Cl}\right), 2.59-2.49(\mathrm{~m}, 2 \mathrm{H}), 2.33-2.19(\mathrm{~m}$, $2 \mathrm{H}), 2.17-1.96(\mathrm{~m}, 2 \mathrm{H}), 1.79-1.70(\mathrm{~m}, 1 \mathrm{H}), 1.24\left(\mathrm{~s}, 6 \mathrm{H}, \mathrm{OC}\left(\mathrm{CH}_{3}\right)_{2}\right), 1.22\left(\mathrm{~s}, 6 \mathrm{H}, \mathrm{OC}\left(\mathrm{CH}_{3}\right)_{2}\right)$; ${ }^{13} \mathrm{C}$ NMR $\left(100 \mathrm{MHz}, \mathrm{CDCl}_{3}\right): \delta 146.8,140.9,137.9,128.7,127.0,126.9,125.8,83.8,56.4$, 43.3, 42.4, 42.2, 29.7, 29.6, 25.3, 24.8, 24.7; IR v 3418 (m), 2974 (m), 1484 (m), 1341 (m), 1141 (s), 1105 (m), 964 (w), 848 (m); HRMS (ESI) calcd. for $\mathrm{C}_{26} \mathrm{H}_{37} \mathrm{NO}_{2} \mathrm{~B}^{+}[\mathrm{M}+\mathrm{H}]^{+}$406.2912; found 406.2902. Carbon attached to boron not observed due to quadrupolar relaxation and another carbon was not resolved at $100 \mathrm{MHz}$. See spectra

$N, N$-Dimethyl-2-((1s,2r)-2-(naphthalen-2-yl)-2-(4,4,5,5-tetramethyl-1,3,2-dioxaborolan2-yl)cyclobutyl)ethan-1-amine hydrochloride (1h)

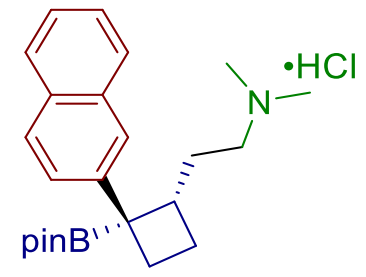

$1 \mathrm{~h}$ 
Following general procedure $\mathbf{C}$, using 2-bromonaphthalene ( $87.0 \mathrm{mg}, 0.420 \mathrm{mmol}, 1.40$ equiv), $n \operatorname{BuLi}(245 \mu \mathrm{L}, 0.390 \mathrm{mmol}, 1.30$ equiv), 4,4,5,5-tetramethyl-2-(1-vinylcyclopropyl)-1,3,2dioxaborolane (4a) (55.3 mg, $0.300 \mathrm{mmol}, 1.00$ equiv), and Eschenmoser's salt (5) (111 mg, $0.600 \mathrm{mmol}, 2.00$ equiv). The crude material was then dissolved in $\mathrm{Et}_{2} \mathrm{O}$ (ca. $0.6 \mathrm{~mL}$ ) and then $2 \mathrm{~N} \mathrm{HCl}(165 \mu \mathrm{L}, 0.330 \mathrm{mmol}, 1.10$ equiv $)$ in $\mathrm{Et}_{2} \mathrm{O}$ was added. A white precipitate was formed, which was collected via a suction filtration (pentane was used for transfer of the compound) and then thoroughly dried under high vacuum to afford $\mathbf{1 h}(117 \mathrm{mg}, 0.282 \mathrm{mmol}, 94 \%,>20: 1$ dr) as an off-white solid. Mp: $196.0-199.5{ }^{\circ} \mathrm{C} ;{ }^{1} \mathrm{H}$ NMR $\left(400 \mathrm{MHz}, \mathrm{CDCl}_{3}\right): \delta 12.59(\mathrm{~s}, 1 \mathrm{H}$, $\left.\left(\mathrm{CH}_{3}\right)_{2} \mathrm{~N} . \mathrm{HCl}\right), 7.80-7.76(\mathrm{~m}, 2 \mathrm{H}, \mathrm{ArH}), 7.74(\mathrm{~d}, J=8.5 \mathrm{~Hz}, 1 \mathrm{H}, \mathrm{ArH}), 7.50-7.36(\mathrm{~m}, 3 \mathrm{H}$, $\operatorname{Ar} H), 7.23(\mathrm{dd}, J=8.5,1.9 \mathrm{~Hz}, 1 \mathrm{H}, \mathrm{Ar} H), 3.11-3.00\left(\mathrm{~m}, 2 \mathrm{H}, \mathrm{CH}_{2}\left(\mathrm{CH}_{3}\right)_{2} \mathrm{~N}\right), 2.85-2.75(\mathrm{~m}$, $\left.6 \mathrm{H},\left(\mathrm{CH}_{3}\right)_{2} \mathrm{~N} . \mathrm{HCl}\right), 2.69-2.56(\mathrm{~m}, 2 \mathrm{H}), 2.43-2.21(\mathrm{~m}, 2 \mathrm{H}), 2.19-2.01(\mathrm{~m}, 2 \mathrm{H}), 1.80-1.72$ $(\mathrm{m}, 1 \mathrm{H}), 1.23\left(\mathrm{~s}, 6 \mathrm{H}, \mathrm{OC}\left(\mathrm{CH}_{3}\right)_{2}\right), 1.20\left(\mathrm{~s}, 6 \mathrm{H}, \mathrm{OC}\left(\mathrm{CH}_{3}\right)_{2}\right) ;{ }^{13} \mathrm{C} \mathrm{NMR}\left(100 \mathrm{MHz}, \mathrm{CDCl}_{3}\right): \delta$ $145.3,133.5,131.5,127.9,127.5,127.5,125.9,125.1,124.9,122.9,83.9,56.4,43.3,42.3$, 42.2, 29.8, 29.6, 25.3, 24.8, 24.7; IR v 3416 (w), 2974 (m), 2637 (w), 1470 (w), 1371 (w), 1314 (w), 1141 (s), 856 (m); HRMS (ESI) calcd. for $\mathrm{C}_{24} \mathrm{H}_{35} \mathrm{BNO}_{2}{ }^{+}[\mathrm{M}+\mathrm{H}]^{+}$380.2759; found 380.2758. Carbon attached to boron not observed due to quadrupolar relaxation. See spectra

\section{$N, N$-Dimethyl-2-((1s,2r)-2-(4,4,5,5-tetramethyl-1,3,2-dioxaborolan-2-yl)-2- vinylcyclobutyl)ethan-1-amine hydrochloride (1i)}

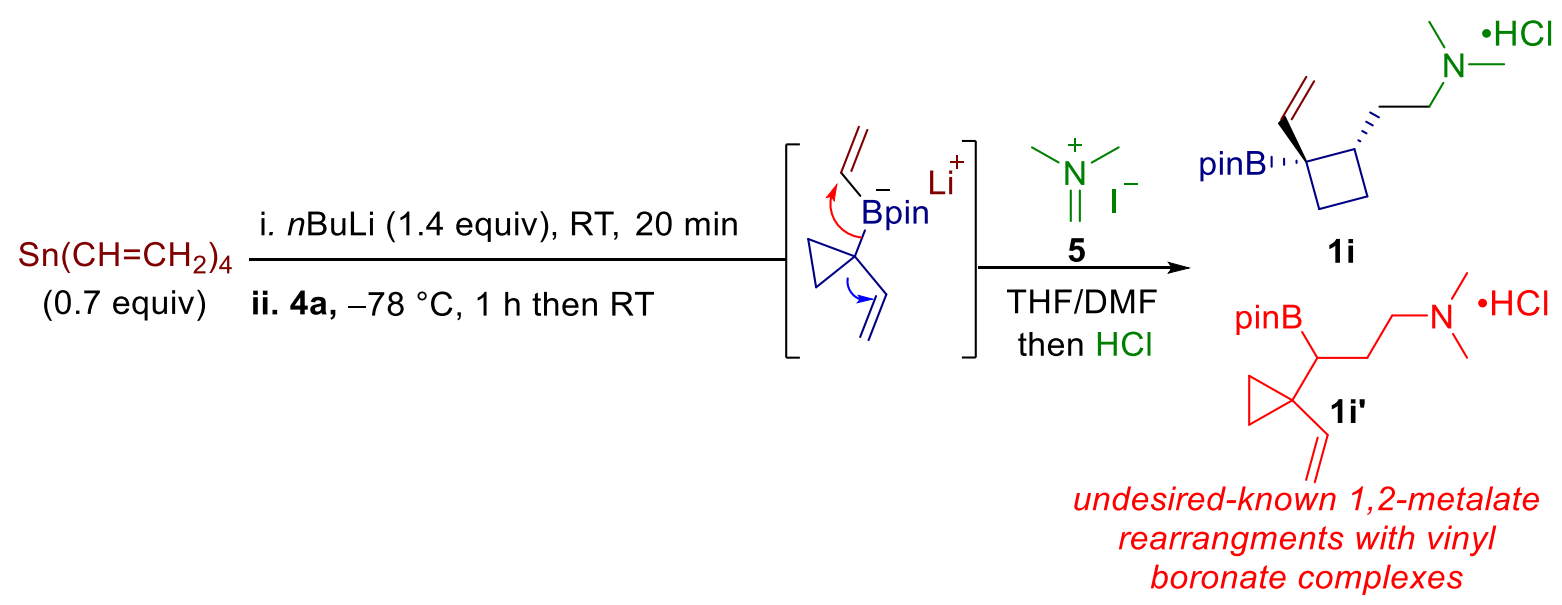

To neat tetravinyltin (48.0 mg, $0.210 \mathrm{mmol}, 0.700$ equiv) at $\mathrm{RT}$ was added $n \mathrm{BuLi}(263 \mu \mathrm{L}$, $0.420 \mathrm{mmol}, 1.40$ equiv) dropwise. The resulting solution was stirred for $30 \mathrm{~min}$ at RT, and then dissolved in THF (1 mL). To a solution of 4,4,5,5-tetramethyl-2-(1-vinylcyclopropyl)1,3,2-dioxaborolane (4a) $(55.3 \mathrm{mg}, 0.300 \mathrm{mmol}, 1.00$ equiv) in THF ( $1 \mathrm{~mL})$ under $\mathrm{N}_{2}$ at -78 ${ }^{\circ} \mathrm{C}$ was added the vinyl lithium solution dropwise. The reaction mixture was stirred at $-78{ }^{\circ} \mathrm{C}$ 
for $1 \mathrm{~h}$ and then warmed to RT and stirred for another $1 \mathrm{~h}$. The reaction mixture was cooled back to $-40{ }^{\circ} \mathrm{C}$ and DMF (2 mL) was added. Eschenmoser's salt (5) (111 mg, $0.600 \mathrm{mmol}$, 2.00 equiv) was then added as a solid at $-40{ }^{\circ} \mathrm{C}$. The reaction was allowed to stir at $-40{ }^{\circ} \mathrm{C}$ for $2 \mathrm{~h}$, then allowed to warm slowly to RT overnight. $0.5 \mathrm{~N} \mathrm{NaOH}(20 \mathrm{~mL})$ was then added, and the mixture was extracted with $\mathrm{Et}_{2} \mathrm{O}(3 \times 20 \mathrm{~mL})$. The combined organic phases were dried over $\mathrm{MgSO}_{4}$, filtered, and concentrated under reduced pressure. The crude material was then dissolved in $\mathrm{Et}_{2} \mathrm{O}(0.5 \mathrm{~mL})$ and then $2 \mathrm{~N} \mathrm{HCl}\left(165 \mu \mathrm{L}, 0.330 \mathrm{mmol}, 1.10\right.$ equiv) in $\mathrm{Et}_{2} \mathrm{O}$ was added. A precipitate was formed, which was collected via a suction filtration (pentane was used for transfer of the compound) and then thoroughly dried under high vacuum to afford $\mathbf{1 i}$ (82.0 $\mathrm{mg}, 0.260 \mathrm{mmol}, 82 \%,>20: 1 \mathrm{dr})$ as a white amorphous solid. ${ }^{1} \mathrm{H} \mathrm{NMR}\left(400 \mathrm{MHz}, \mathrm{CDCl}_{3}\right): \delta$ $12.23\left(\mathrm{~s}, 1 \mathrm{H},\left(\mathrm{CH}_{3}\right)_{2} \mathrm{~N} . H \mathrm{Cl}\right), 5.91\left(\mathrm{dd}, J=17.3,10.5 \mathrm{~Hz}, 1 \mathrm{H}, \mathrm{CCH}=\mathrm{CH}_{2}\right), 4.97-4.76(\mathrm{~m}, 2 \mathrm{H}$, $\left.\mathrm{CCH}=\mathrm{CH}_{2}\right), 3.10-2.98(\mathrm{~m}, 1 \mathrm{H}), 2.95-2.86(\mathrm{~m}, 1 \mathrm{H}), 2.86-2.67\left(\mathrm{~m}, 6 \mathrm{H},(\mathrm{CH})_{2} \mathrm{~N} . \mathrm{HCl}\right), 2.20$ - $1.96(\mathrm{~m}, 4 \mathrm{H}), 1.96-1.87(\mathrm{~m}, 1 \mathrm{H}), 1.86-1.74(\mathrm{~m}, 1 \mathrm{H}), 1.68-1.51(\mathrm{~m}, 1 \mathrm{H}), 1.26(\mathrm{~s}, 12 \mathrm{H}$, Bpin); ${ }^{13} \mathrm{C}$ NMR (100 MHz, $\left.\mathrm{CDCl}_{3}\right): \delta$ 143.9, 111.5, 83.7, 56.3, 43.6, 42.3, 42.0, 29.1, 27.5, 25.0, 24.8, 24.7; IR v 3399 (s), 2974 (s), 2938 (m), 2670 (w), 1445 (m), 1371 (s), 1313 (s), 1140 (s), 1008 (w), 966 (m); HRMS (ESI) calcd. for $\mathrm{C}_{16} \mathrm{H}_{31} \mathrm{BNO}_{2}{ }^{+}[\mathrm{M}+\mathrm{H}]^{+}$280.2445; found 280.2446. Carbon attached to boron not observed due to quadrupolar relaxation. See spectra

\section{$N, N$-Dimethyl-2-((1s,2r)-2-((E)-oct-1-en-1-yl)-2-(4,4,5,5-tetramethyl-1,3,2-dioxaborolan-}

\section{2-yl)cyclobutyl)ethan-1-amine (1j)}

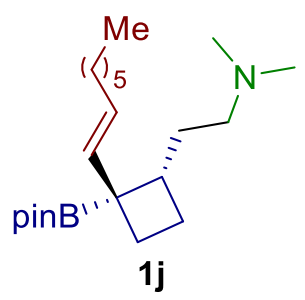

Following general procedure C, using (E)-1-iodooct-1-ene (100 mg, $0.420 \mathrm{mmol}, 1.40$ equiv), $t$ BuLi (525 $\mu \mathrm{L}, 0.840 \mathrm{mmol}, 2.80$ equiv), 4,4,5,5-tetramethyl-2-(1-vinylcyclopropyl)-1,3,2dioxaborolane (4a) (55.3 mg, $0.300 \mathrm{mmol}, 1.00$ equiv), and Eschenmoser's salt (5) (111 mg, $0.600 \mathrm{mmol}, 2.00$ equiv). The crude material was purified by flash chromatography using MeOH:DCM 1:12 as mobile phase to afford $1 \mathbf{j}(66.0 \mathrm{mg}, 0.182 \mathrm{mmol}, 61 \%,>20: 1 \mathrm{dr})$ as a colorless oil. TLC (MeOH:DCM, 1:10 v/v): $\mathrm{R}_{\mathrm{f}}=0.24, \mathrm{KMnO}_{4} ;{ }^{1} \mathrm{H}$ NMR (400 MHz, $\left.\mathrm{CDCl}_{3}\right)$ : $\delta 5.62(\mathrm{dt}, J=15.5,1.4 \mathrm{~Hz}, 1 \mathrm{H}, \mathrm{BCC} H=\mathrm{CH}), 5.25(\mathrm{dt}, J=15.5,6.8 \mathrm{~Hz}, 1 \mathrm{H}, \mathrm{BCCH}=\mathrm{CH}), 2.20$ $\left.\left(\mathrm{s}, 6 \mathrm{H}, \mathrm{CH}_{3}\right)_{2} \mathrm{~N}\right), 2.19-2.16(\mathrm{~m}, 2 \mathrm{H}), 2.15-2.04(\mathrm{~m}, 2 \mathrm{H}), 1.98(\mathrm{qt}, J=7.0,1.8 \mathrm{~Hz}, 2 \mathrm{H}), 1.91$ 
$(\mathrm{dtd}, J=10.4,8.3,2.4 \mathrm{~Hz}, 1 \mathrm{H}), 1.84-1.71(\mathrm{~m}, 3 \mathrm{H}), 1.61-1.53(\mathrm{~m}, 1 \mathrm{H}), 1.54-1.45(\mathrm{~m}, 1 \mathrm{H})$, $1.34-1.26(\mathrm{~m}, 7 \mathrm{H}), 1.25$ (s, 12H, Bpin), 0.87 (t, $\left.J=7.0 \mathrm{~Hz}, 3 \mathrm{H}, \mathrm{CH}_{2} \mathrm{CH}_{3}\right) ;{ }^{13} \mathrm{C}$ NMR (100 $\left.\mathrm{MHz}, \mathrm{CDCl}_{3}\right): \delta 136.7,126.4,83.0,58.0,45.5,44.3,32.9,32.7,31.8,29.8,28.7,27.3,27.2$, 25.1, 24.9, 24.8, 14.1; IR v 2972 (s), 2923 (s), 2761 (w), 1460 (m), 1371(m), 1143 (m), 1056 (s), 965 (w); HRMS (ESI) calcd. for $\mathrm{C}_{22} \mathrm{H}_{43} \mathrm{BNO}_{2}{ }^{+}[\mathrm{M}+\mathrm{H}]^{+}$364.3385; found 364.3389. Carbon attached to boron not observed due to quadrupolar relaxation. See spectra

\section{$N, N$-Dimethyl-2-((1s,2r)-2-methyl-2-(4,4,5,5-tetramethyl-1,3,2-dioxaborolan-2- yl)cyclobutyl)ethan-1-amine hydrochloride (1k)}

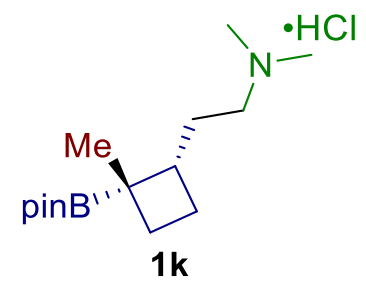

Following general procedure B, using 4,4,5,5-tetramethyl-2-(1-vinylcyclopropyl)-1,3,2dioxaborolane (4a) (55.3 mg, $0.300 \mathrm{mmol}, 1.00$ equiv), MeLi (243 $\mu \mathrm{L}, 0.390 \mathrm{mmol}, 1.61 \mathrm{M}$ in $\mathrm{Et}_{2} \mathrm{O}, 1.30$ equiv), and Eschenmoser's salt (5) $(111 \mathrm{mg}, 0.600 \mathrm{mmol}, 2.00$ equiv). The crude material was then dissolved in pentane $(0.6 \mathrm{~mL})$ and then $2 \mathrm{~N} \mathrm{HCl}(165 \mu \mathrm{L}, 0.330 \mathrm{mmol}, 1.10$ equiv) in $\mathrm{Et}_{2} \mathrm{O}$ was added. A white precipitate was formed, which was collected via a suction filtration (pentane was used for transfer of the compound) and then thoroughly dried under high vacuum to afford $1 \mathbf{k}(56.0 \mathrm{mg}, 0.185 \mathrm{mmol}, 62 \%,>20: 1 \mathrm{dr})$ as a white amorphous solid. ${ }^{1} \mathrm{H}$ NMR $\left(400 \mathrm{MHz}, \mathrm{CDCl}_{3}\right): \delta 12.38\left(\mathrm{~s}, 1 \mathrm{H},\left(\mathrm{CH}_{3}\right)_{2} \mathrm{~N} . \mathrm{HCl}\right), 3.08-2.96(\mathrm{~m}, 1 \mathrm{H}), 2.95-2.86$ $(\mathrm{m}, 1 \mathrm{H}), 2.84-2.75\left(\mathrm{~m}, 6 \mathrm{H},\left(\mathrm{CH}_{3}\right)_{2} \mathrm{~N} . H \mathrm{HCl}\right), 2.10(\mathrm{t}, J=9.3 \mathrm{~Hz}, 1 \mathrm{H}), 2.04-1.73(\mathrm{~m}, 4 \mathrm{H}), 1.63$ - 1.45 (m, 2H), 1.28 (s, 12H, Bpin), 1.10 (s, 3H, BCCH $\left.\left.{ }_{3}\right) ;{ }^{13} \mathrm{C} \mathrm{NMR} \mathrm{(100} \mathrm{MHz,} \mathrm{CDCl}_{3}\right): \delta 83.3$, 56.2, 44.1, 43.1, 42.1, 29.7, 28.8, 25.6, 25.1, 24.7, 24.5; IR v 3399 (m), 2973 (m), 2938 (m), 1454 (m), 1380 (m), 1307 (m), 1138 (s), 1014 (w), 848 (m); HRMS (ESI) calcd. for $\mathrm{C}_{15} \mathrm{H}_{31} \mathrm{NO}_{2} \mathrm{~B}^{+}[\mathrm{M}+\mathrm{H}]^{+}$268.2442; found 268.2445. Carbon attached to boron not observed due to quadrupolar relaxation. $\underline{\text { See spectra }}$ 


\section{2-((1s,2r)-2-Butyl-2-(4,4,5,5-tetramethyl-1,3,2-dioxaborolan-2-yl)cyclobutyl)- $N, N$ -}

dimethylethan-1-amine hydrochloride (11)

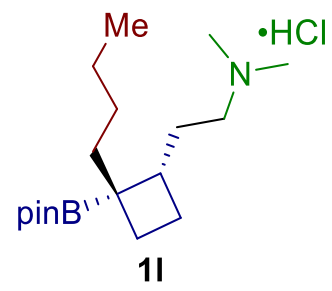

Following general procedure B, using 4,4,5,5-tetramethyl-2-(1-vinylcyclopropyl)-1,3,2dioxaborolane (4a) (55.3 mg, $0.300 \mathrm{mmol}, 1.00$ equiv), ${ }^{n} \mathrm{BuLi}(245 \mu \mathrm{L}, 0.390 \mathrm{mmol}, 1.60 \mathrm{M}$ in hexanes, 1.30 equiv), and Eschenmoser's salt (5) (111 mg, $0.600 \mathrm{mmol}, 2.00$ equiv). The crude material was then dissolved in pentane $(0.6 \mathrm{~mL})$ and then $2 \mathrm{~N} \mathrm{HCl}(165 \mu \mathrm{L}, 0.330 \mathrm{mmol}$, 1.10 equiv) in $\mathrm{Et}_{2} \mathrm{O}$ was added. A white precipitate was formed, which was collected via a suction filtration (pentane was used for transfer of the compound) and then thoroughly dried under high vacuum to afford $11(75.0 \mathrm{mg}, 0.243 \mathrm{mmol}, 81 \%,>20: 1 \mathrm{dr})$ as a white amorphous solid. ${ }^{1} \mathrm{H}$ NMR $\left(400 \mathrm{MHz}, \mathrm{CDCl}_{3}\right): \delta 12.38\left(\mathrm{~s}, 1 \mathrm{H},\left(\mathrm{CH}_{3}\right)_{2} \mathrm{~N} . \mathrm{HCl}\right), 3.12-3.02(\mathrm{~m}, 1 \mathrm{H}), 2.94-$ $2.84(\mathrm{~m}, 1 \mathrm{H}), 2.82-2.74\left(\mathrm{~m}, 6 \mathrm{H},\left(\mathrm{CH}_{3}\right)_{2} \mathrm{~N} . \mathrm{HCl}\right), 2.15-2.07(\mathrm{~m}, 1 \mathrm{H}), 2.04-1.80(\mathrm{~m}, 4 \mathrm{H})$, $1.64-1.43(\mathrm{~m}, 3 \mathrm{H}), 1.38-0.97(\mathrm{~m}, 17 \mathrm{H}), 0.88\left(\mathrm{t}, J=7.3 \mathrm{~Hz}, 3 \mathrm{H}, \mathrm{CH}_{2} \mathrm{CH}_{3}\right) ;{ }^{13} \mathrm{C} \mathrm{NMR}(100$ $\left.\mathrm{MHz} \mathrm{CDCl}_{3}\right): \delta 83.4,56.4,43.2,43.1,42.0,40.9,29.2,28.8,27.8,25.4,24.8,24.7,23.2,14.0$; IR v 3418 (w), 2957 (m), 2925 (m), 2480 (m), 1457 (w), 1385 (s), 1212 (w), 1139 (s), 957 (m); HRMS (ESI) calcd. for $\mathrm{C}_{18} \mathrm{H}_{37} \mathrm{NO}_{2} \mathrm{~B}^{+}[\mathrm{M}+\mathrm{H}]^{+}$310.2912; found 310.2904. Carbon attached to boron not observed due to quadrupolar relaxation. See spectra

\section{2-((1s,2r)-2-Isopropyl-2-(4,4,5,5-tetramethyl-1,3,2-dioxaborolan-2-yl)cyclobutyl)- $N, N$ -} dimethylethan-1-amine hydrochloride (1m)

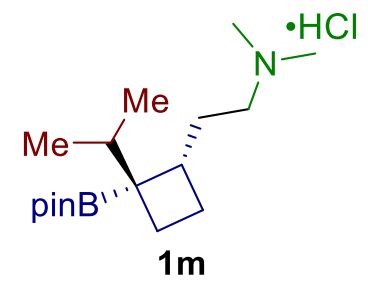

Following general procedure B, using 4,4,5,5-tetramethyl-2-(1-vinylcyclopropyl)-1,3,2dioxaborolane (4a) (55.3 mg, $0.300 \mathrm{mmol}, 1.00$ equiv), ${ }^{i} \operatorname{PrLi}(558 \mu \mathrm{L}, 0.390 \mathrm{mmol}, 0.7 \mathrm{M}$ in THF, 1.30 equiv), and Eschenmoser's salt (5) (111 mg, $0.600 \mathrm{mmol}, 2.00$ equiv). The crude material was then dissolved in pentane $(0.6 \mathrm{~mL})$ and then $2 \mathrm{~N} \mathrm{HCl}(165 \mu \mathrm{L}, 0.330 \mathrm{mmol}, 1.10$ equiv) in $\mathrm{Et}_{2} \mathrm{O}$ was added. A white precipitate was formed, which was collected via a suction 
filtration (pentane was used for transfer of the compound) and then thoroughly dried under high vacuum to afford $\mathbf{1 m}(78.0 \mathrm{mg}, 0.235 \mathrm{mmol}, 78 \%,>20: 1 \mathrm{dr})$ as an off-white solid. Mp: 184.5 - $187.3{ }^{\circ} \mathrm{C} ;{ }^{1} \mathrm{H}$ NMR $\left(400 \mathrm{MHz}, \mathrm{CDCl}_{3}\right)$ : $\delta 12.38\left(\mathrm{~s}, 1 \mathrm{H},\left(\mathrm{CH}_{3}\right)_{2} \mathrm{~N} . \mathrm{HCl}\right), 3.01-2.91(\mathrm{~m}$, $1 \mathrm{H}), 2.94-2.84(\mathrm{~m}, 1 \mathrm{H}), 2.78-2.72\left(\mathrm{~m}, 6 \mathrm{H},\left(\mathrm{CH}_{3}\right)_{2} \mathrm{~N} . H \mathrm{HCl}\right), 2.09-2.00(\mathrm{~m}, 1 \mathrm{H}), 1.98-1.82$ (m, 4H), $1.65-1.57\left(\mathrm{~m}, 1 \mathrm{H}, \mathrm{CHCH}_{3}\right), 1.56-1.36(\mathrm{~m}, 2 \mathrm{H}), 1.26$ (s, 12H, Bpin), 0.85 (d, J= $\left.6.8 \mathrm{~Hz}, 3 \mathrm{H}, \mathrm{CHCH}_{3}\right), 0.80\left(\mathrm{~d}, J=6.8 \mathrm{~Hz}, 3 \mathrm{H}, \mathrm{CHCH}_{3}\right) ;{ }^{13} \mathrm{C} \mathrm{NMR}\left(100 \mathrm{MHz}, \mathrm{CDCl}_{3}\right): \delta 83.2$, 56.4, 42.9, 42.2, 40.3, 37.8, 29.6, 26.2, 25.3, 25.0, 24.0, 19.2, 18.6; IR v $3416(\mathrm{w}), 2955$ (m), 2868 (w), 1458 (w), 1385 (m), 1305 (m), 1140 (s), 1048 (w), 955 (w); HRMS (ESI) calcd. for $\mathrm{C}_{17} \mathrm{H}_{35} \mathrm{BNO}_{2}{ }^{+}[\mathrm{M}+\mathrm{H}]^{+}$296.2755; found 296.2752. Carbon attached to boron not observed due to quadrupolar relaxation. See spectra

\section{2-((1s,2r)-2-Cyclopropyl-2-(4,4,5,5-tetramethyl-1,3,2-dioxaborolan-2-yl)cyclobutyl)-}

\section{$N, N$-dimethylethan-1-amine hydrochloride (1n)}

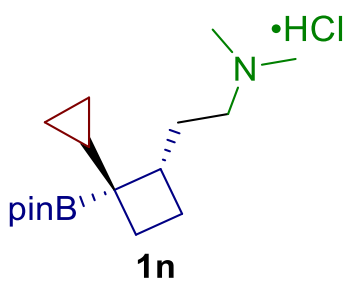

Following general procedure $\mathbf{C}$, using bromocyclopropane (44.0 mg, $0.360 \mathrm{mmol}, 1.20$ equiv), ${ }^{t} \mathrm{BuLi}$ (424 $\mu \mathrm{L}, 0.720 \mathrm{mmol}, 1.70 \mathrm{M}$ in pentane, 2.40 equiv), 4,4,5,5-tetramethyl-2-(1vinylcyclopropyl)-1,3,2-dioxaborolane (4a) $(55.3 \mathrm{mg}, 0.300 \mathrm{mmol}, 1.00$ equiv), and Eschenmoser's salt (5) $(111 \mathrm{mg}, 0.600 \mathrm{mmol}, 2.00$ equiv). The crude material was then dissolved in pentane $(0.5 \mathrm{~mL})$ and then $2 \mathrm{~N} \mathrm{HCl}\left(165 \mu \mathrm{L}, 0.330 \mathrm{mmol}, 1.10\right.$ equiv) in $\mathrm{Et}_{2} \mathrm{O}$ was added. A white precipitate was formed, which was collected via a suction filtration (pentane was used for transfer of the compound) and then thoroughly dried under high vacuum to afford 1n $(72.0 \mathrm{mg}, 0.218 \mathrm{mmol}, 73 \%, 12: 1 \mathrm{dr})$ as a white amorphous solid. ${ }^{1} \mathrm{H}$ NMR $(400 \mathrm{MHz}$, $\left.\mathrm{CDCl}_{3}\right): \delta 12.18\left(\mathrm{~s}, 1 \mathrm{H},\left(\mathrm{CH}_{3}\right)_{2} \mathrm{~N} . H \mathrm{HCl}\right), 3.16-3.05(\mathrm{~m}, 1 \mathrm{H}), 3.00-2.87(\mathrm{~m}, 1 \mathrm{H}), 2.80-2.74$ $\left(\mathrm{m}, 6 \mathrm{H},\left(\mathrm{CH}_{3}\right)_{2} \mathrm{~N} . H \mathrm{Cl}\right), 2.10-1.72(\mathrm{~m}, 5 \mathrm{H}), 1.52-1.39(\mathrm{~m}, 1 \mathrm{H}), 1.37-1.29(\mathrm{~m}, 1 \mathrm{H}), 1.26(\mathrm{~s}$, $\left.6 \mathrm{H}, \mathrm{OC}\left(\mathrm{CH}_{3}\right)_{2}\right), 1.25\left(\mathrm{~s}, 6 \mathrm{H}, \mathrm{OC}\left(\mathrm{CH}_{3}\right)_{2}\right), 0.85-0.67(\mathrm{~m}, 1 \mathrm{H}), 0.38-0.16(\mathrm{~m}, 3 \mathrm{H}), 0.16$ - $0.02(\mathrm{~m}, 1 \mathrm{H}) ;{ }^{13} \mathrm{C}$ NMR $\left(100 \mathrm{MHz}, \mathrm{CDCl}_{3}\right): \delta 83.4,56.5,43.4,41.9,41.9,29.0,25.1,24.7$, 24.5, 23.3, 17.3, 1.7, 1.0; IR v 3413 (m), 2974 (m), 2474 (w), 1469 (w), 1372 (m), 1342 (m), 1141 (s), 1013 (w), 844 (w); HRMS (ESI) calcd. for $\mathrm{C}_{17} \mathrm{H}_{33} \mathrm{BNO}_{2}{ }^{+}[\mathrm{M}+\mathrm{H}]^{+}$293.2599; found 294.2590. Carbon attached to boron not observed due to quadrupolar relaxation. See spectra 


\section{$N, N$-Dimethyl-2-((1s,2r)-1-(4,4,5,5-tetramethyl-1,3,2-dioxaborolan-2-yl)-[1,1'-}

bi(cyclobutan)]-2-yl)ethan-1-amine hydrochloride (10)

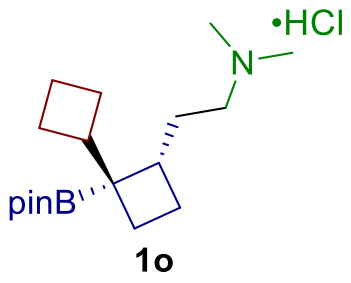

Following general procedure $\mathbf{C}$, using bromocyclobutane (49.0 mg, $0.360 \mathrm{mmol}, 1.20$ equiv), ${ }^{t} \mathrm{BuLi}(424 \mu \mathrm{L}, 0.720 \mathrm{mmol}, 1.70 \mathrm{M}$ in pentane, 2.40 equiv), 4,4,5,5-tetramethyl-2-(1vinylcyclopropyl)-1,3,2-dioxaborolane (4a) $(55.3 \mathrm{mg}, 0.300 \mathrm{mmol}, 1.00$ equiv), and Eschenmoser's salt (5) $(111 \mathrm{mg}, 0.600 \mathrm{mmol}, 2.00$ equiv). The crude material was then dissolved in pentane $(0.5 \mathrm{~mL})$ and then $2 \mathrm{~N} \mathrm{HCl}(165 \mu \mathrm{L}, 0.330 \mathrm{mmol}, 1.10$ equiv $)$ in $\mathrm{Et}_{2} \mathrm{O}$ was added. A white precipitate was formed, which was collected via a suction filtration (pentane was used for transfer of the compound) and then thoroughly dried under high vacuum to afford $10(65.0 \mathrm{mg}, 0.189 \mathrm{mmol}, 63 \%, 13: 1 \mathrm{dr})$ as a white amorphous solid. ${ }^{1} \mathrm{H}$ NMR (400 MHz, $\left.\mathrm{CDCl}_{3}\right): \delta 12.20\left(\mathrm{~s}, 1 \mathrm{H},\left(\mathrm{CH}_{3}\right)_{2} \mathrm{~N} . H \mathrm{Cl}\right), 3.23-2.96(\mathrm{~m}, 2 \mathrm{H}), 2.79-2.74\left(\mathrm{~m}, 6 \mathrm{H},\left(\mathrm{CH}_{3}\right)_{2} \mathrm{~N} . H \mathrm{Cl}\right)$, $2.49-2.33(\mathrm{~m}, 1 \mathrm{H}), 2.00-1.84(\mathrm{~m}, 7 \mathrm{H}), 1.81-1.72(\mathrm{~m}, 4 \mathrm{H}), 1.69-1.57(\mathrm{~m}, 2 \mathrm{H}), 1.25(\mathrm{~s}$, $\left.6 \mathrm{H}, \mathrm{OC}\left(\mathrm{CH}_{3}\right)_{2}\right), 1.25\left(\mathrm{~s}, 6 \mathrm{H}, \mathrm{OC}\left(\mathrm{CH}_{3}\right)_{2}\right) ;{ }^{13} \mathrm{C} \mathrm{NMR}\left(100 \mathrm{MHz}, \mathrm{CDCl}_{3}\right)$ : $\delta 83.3,56.4,43.3,42.0$, 41.3, 37.8, 29.1, 25.2, 24.8, 24.6, 24.4, 23.5, 22.2, 17.7; IR v 3384 (m), 2970 (s), $2864(\mathrm{w})$, 1638 (w), 1470 (m), 1372 (s), 1307 (s), 1141 (s), 995 (w), 854 (w); HRMS (ESI) calcd. for $\mathrm{C}_{18} \mathrm{H}_{35} \mathrm{BNO}_{2}{ }^{+}[\mathrm{M}+\mathrm{H}]^{+}$308.2755; found 308.2746. Carbon attached to boron not observed due to quadrupolar relaxation. See spectra

\section{$N, N$-Dimethyl-2-((1s,2r)-2-phenyl-2-(4,4,5,5-tetramethyl-1,3,2-dioxaborolan-2-} yl)cyclobutyl)ethan-1-amine hydrochloride (1a)

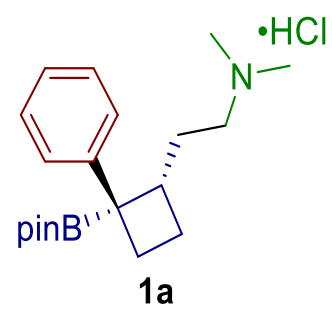

Following general procedure D, using $\mathrm{LiCl}(19.0 \mathrm{mg}, 0.450 \mathrm{mmol}, 1.50$ equiv), phenylmagnesium bromide (390 $\mu \mathrm{L}, 0.390 \mathrm{mmol}, 1 \mathrm{M}$ in THF, 1.30 equiv), 4,4,5,5tetramethyl-2-(1-vinylcyclopropyl)-1,3,2-dioxaborolane (4a) $(55.3 \mathrm{mg}, 0.300 \mathrm{mmol}, 1.00$ 
equiv), and Eschenmoser's salt (5) (111 mg, $0.600 \mathrm{mmol}, 2.00$ equiv). The crude material was then dissolved in $\mathrm{Et}_{2} \mathrm{O}$ (ca. $\left.0.6 \mathrm{~mL}\right)$ and then $2 \mathrm{~N} \mathrm{HCl}\left(165 \mu \mathrm{L}, 0.330 \mathrm{mmol}, 1.10\right.$ equiv) in $\mathrm{Et}_{2} \mathrm{O}$ was added. A white precipitate was formed, which was collected via a suction filtration (pentane was used for transfer of the compound) and then thoroughly dried under high vacuum to afford 1a (90.0 mg, $0.273 \mathrm{mmol}, 91 \%,>20: 1 \mathrm{dr}$ ) as an off-white solid. Mp: $218.2-220.5$ ${ }^{\circ} \mathrm{C} ;{ }^{1} \mathrm{H}$ NMR $\left(400 \mathrm{MHz}, \mathrm{CDCl}_{3}\right): \delta 12.56\left(\mathrm{~s}, 1 \mathrm{H},\left(\mathrm{CH}_{3}\right)_{2} \mathrm{~N} . \mathrm{HCl}\right), 7.36-7.22(\mathrm{~m}, 2 \mathrm{H}, \mathrm{ArH})$, $7.18-7.00(\mathrm{~m}, 3 \mathrm{H}, \mathrm{ArH}), 3.09-2.97\left(\mathrm{~m}, 2 \mathrm{H}, \mathrm{CH}_{2}\left(\mathrm{CH}_{3}\right)_{2} \mathrm{~N}\right), 2.82\left(\right.$ br s, $\left.6 \mathrm{H},\left(\mathrm{CH}_{3}\right)_{2} \mathrm{~N} . \mathrm{HCl}\right)$, $2.60-2.48(\mathrm{~m}, 2 \mathrm{H}), 2.30-2.22(\mathrm{~m}, 2 \mathrm{H}), 2.11-2.01(\mathrm{~m}, 2 \mathrm{H}), 1.77-1.68(\mathrm{~m}, 1 \mathrm{H}), 1.24(\mathrm{~s}$, $\left.6 \mathrm{H}, \mathrm{OC}\left(\mathrm{CH}_{3}\right)_{2}\right), 1.22\left(\mathrm{~s}, 6 \mathrm{H}, \mathrm{OC}\left(\mathrm{CH}_{3}\right)_{2}\right) ;{ }^{13} \mathrm{C} \mathrm{NMR}\left(100 \mathrm{MHz}, \mathrm{CDCl}_{3}\right): \delta 147.7,128.3,125.3$, 125.1, 83.8, 56.3, 43.2, 42.2, 42.2, 29.7, 29.5, 25.3, 24.8, 24.7; IR v 3419 (m), 2974 (m), 2937 (w), 1479 (w), 1444 (w), 1342 (s), 1312 (s), 1141 (s), 1105 (m), 954 (w); HRMS (ESI) calcd. for $\mathrm{C}_{20} \mathrm{H}_{33} \mathrm{NO}_{2} \mathrm{~B}^{+}[\mathrm{M}+\mathrm{H}]^{+} 330.2599$; found 330.2589. Carbon attached to boron not observed due to quadrupolar relaxation. See spectra

\section{2-((1s,2s)-2-Benzyl-2-(4,4,5,5-tetramethyl-1,3,2-dioxaborolan-2-yl)cyclobutyl)- $N, N$ - dimethylethan-1-amine hydrochloride (1p)}

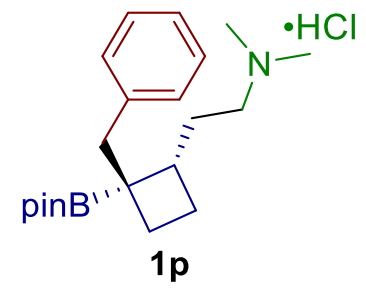

Following general procedure $\mathbf{D}$, using $\mathrm{LiCl}(19.0 \mathrm{mg}, 0.450 \mathrm{mmol}, 1.50$ equiv), benzylmagnesium chloride $(390 \mu \mathrm{L}, 0.390 \mathrm{mmol}, 1 \mathrm{M}$ in THF, 1.30 equiv), 4,4,5,5tetramethyl-2-(1-vinylcyclopropyl)-1,3,2-dioxaborolane (4a) $(55.3 \mathrm{mg}, 0.300 \mathrm{mmol}, 1.00$ equiv), and Eschenmoser's salt (5) (111 $\mathrm{mg}, 0.600 \mathrm{mmol}, 2.00$ equiv). The crude material was then dissolved in pentane $(0.6 \mathrm{~mL})$ and then $2 \mathrm{~N} \mathrm{HCl}(165 \mu \mathrm{L}, 0.330 \mathrm{mmol}, 1.10$ equiv $)$ in $\mathrm{Et}_{2} \mathrm{O}$ was added. A white precipitate was formed, which was collected via a suction filtration (pentane was used for transfer of the compound) and then thoroughly dried under high vacuum to afford $1 p(92.0 \mathrm{mg}, 0.242 \mathrm{mmol}, 81 \%,>20: 1 \mathrm{dr})$ as a light-yellow amorphous solid. ${ }^{1} \mathrm{H}$ NMR (400 MHz, $\left.\mathrm{CDCl}_{3}\right): \delta 12.07\left(\mathrm{~s}, 1 \mathrm{H},\left(\mathrm{CH}_{3}\right)_{2} \mathrm{~N} . \mathrm{HCl}\right), 7.27$ - $7.22(\mathrm{~m}, 2 \mathrm{H}, \mathrm{ArH}), 7.22-7.15(\mathrm{~m}$, $3 \mathrm{H}, \operatorname{Ar} H), 2.95(\mathrm{~d}, J=13.3 \mathrm{~Hz}, 1 \mathrm{H}), 2.72-2.60(\mathrm{~m}, 7 \mathrm{H}), 2.57-2.48(\mathrm{~m}, 1 \mathrm{H}), 2.21-2.06(\mathrm{~m}$, , 3H), $2.04-1.97(\mathrm{~m}, 1 \mathrm{H}), 1.96-1.82(\mathrm{~m}, 2 \mathrm{H}), 1.81-1.75(\mathrm{~m}, 1 \mathrm{H}), 1.63(\mathrm{p}, J=9.9 \mathrm{~Hz}, 1 \mathrm{H})$, $1.22\left(\mathrm{~s}, 6 \mathrm{H}, \mathrm{OC}\left(\mathrm{CH}_{3}\right)_{2}\right), 1.21\left(\mathrm{~s}, 6 \mathrm{H}, \mathrm{OC}\left(\mathrm{CH}_{3}\right)_{2}\right) ;{ }^{13} \mathrm{C} \mathrm{NMR}\left(100 \mathrm{MHz}, \mathrm{CDCl}_{3}\right): \delta 140.4,129.4$, 128.1, 126.1, 83.5, 56.2, 46.1, 43.5, 41.9, 41.5, 29.2, 28.7, 25.1, 24.9, 24.7; IR v 3399 (m), 
2973 (s), 2568 (w), 1478 (w), 1386 (s), 1141 (m), 1055 (w), 963 (w), 849 (w); HRMS (ESI) calcd. for $\mathrm{C}_{21} \mathrm{H}_{35} \mathrm{BNO}_{2}{ }^{+}[\mathrm{M}+\mathrm{H}]^{+}$344.2755; found 344.2745. Carbon attached to boron not observed due to quadrupolar relaxation. See spectra

\section{2-((1s,2s)-2-(2-(1,3-Dioxan-2-yl)ethyl)-2-(4,4,5,5-tetramethyl-1,3,2-dioxaborolan-2-} yl)cyclobutyl)- $N, N$-dimethylethan-1-amine (1q)

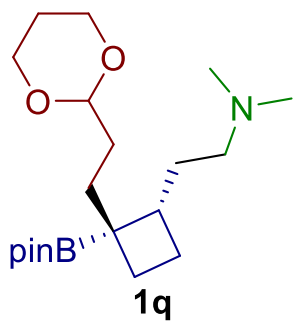

Following general procedure $\mathbf{D}$, using $\mathrm{LiCl}$ (19.0 mg, $0.450 \mathrm{mmol}, 1.50$ equiv), (2-(1,3-dioxan2-yl)ethyl)magnesium bromide (780 $\mu \mathrm{L}, 0.390 \mathrm{mmol}, 0.5 \mathrm{M}$ in THF, 1.30 equiv), 4,4,5,5tetramethyl-2-(1-vinylcyclopropyl)-1,3,2-dioxaborolane (4a) (55.3 mg, $0.300 \mathrm{mmol}, 1.00$ equiv), and Eschenmoser's salt (5) (111 mg, $0.600 \mathrm{mmol}, 2.00$ equiv). The crude material was purified by flash chromatography using MeOH:DCM 1:12 as mobile phase to afford 1q (67.0 $\mathrm{mg}, 0.182 \mathrm{mmol}, 61 \%$, >20:1 dr) as a colorless oil. TLC (MeOH:DCM, 1:10 v/v): $\mathrm{R}_{\mathrm{f}}=0.24$, $\mathrm{KMnO}_{4} ;{ }^{1} \mathrm{H} \mathrm{NMR}\left(400 \mathrm{MHz}, \mathrm{CDCl}_{3}\right.$ ): $\delta 4.45$ (t, $J=5.2 \mathrm{~Hz}, 1 \mathrm{H}, \mathrm{OCHO}$ ), 4.09 (ddt, $J=9.6$, 5.0, $\left.1.5 \mathrm{~Hz}, 2 \mathrm{H}, \mathrm{OCH}_{2}\right), 3.81-3.64\left(\mathrm{~m}, 2 \mathrm{H}, \mathrm{OCH}_{2}\right), 2.18\left(\mathrm{~s}, 6 \mathrm{H},\left(\mathrm{CH}_{3}\right)_{2} \mathrm{~N}\right), 2.17-2.11(\mathrm{~m}$, $2 \mathrm{H}), 2.08-2.00(\mathrm{~m}, 2 \mathrm{H}), 1.98-1.87(\mathrm{~m}, 2 \mathrm{H}), 1.79-1.62(\mathrm{~m}, 2 \mathrm{H}), 1.61-1.55(\mathrm{~m}, 1 \mathrm{H}), 1.52$ $-1.44(\mathrm{~m}, 2 \mathrm{H}), 1.42-1.36(\mathrm{~m}, 1 \mathrm{H}), 1.33-1.28(\mathrm{~m}, 1 \mathrm{H}), 1.27-1.20(\mathrm{~m}, 14 \mathrm{H}) ;{ }^{13} \mathrm{C} \mathrm{NMR}(100$ $\left.\mathrm{MHz}, \mathrm{CDCl}_{3}\right): \delta 102.9,83.1,66.9,58.0,45.5,43.9,34.9,33.1,32.2,29.7,27.3,25.9,25.1$, 25.0, 24.8; IR v 2925 (s), 2852 (m), 2762 (w), 1852 (w), 1461 (m), 1385 (s), 1306 (m), 1214 (m), 1142 (s), 1081 (w), $846(\mathrm{~m})$; HRMS (ESI) calcd. for $\mathrm{C}_{20} \mathrm{H}_{39} \mathrm{BNO}_{4}{ }^{+}[\mathrm{M}+\mathrm{H}]^{+}$368.2970; found 368.2985. Carbon attached to boron not observed due to quadrupolar relaxation. See spectra

1-(2-((1s,2r)-2-(3,5-Bis(trifluoromethyl)phenyl)-2-(4,4,5,5-tetramethyl-1,3,2dioxaborolan-2-yl)cyclobutyl)ethyl)pyrrolidine (1r)

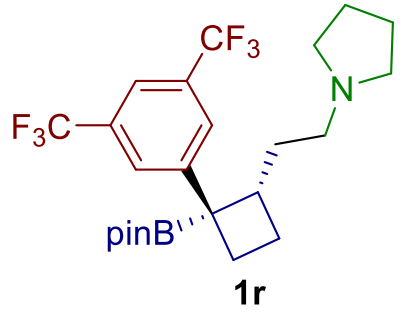


Following general procedure E, using 1-bromo-3,5-bis(trifluoromethyl)benzene (123 mg, 0.420 mmol, 1.40 equiv), $n \mathrm{BuLi}(245 \mu \mathrm{L}, 0.390 \mathrm{mmol}, 1.30$ equiv), 4,4,5,5-tetramethyl-2-(1vinylcyclopropyl)-1,3,2-dioxaborolane (4a) (55.3 $\mathrm{mg}, 0.300 \mathrm{mmol}, 1.00$ equiv), and pyrrolidine derived iminium salt. $^{2}(127 \mathrm{mg}, 0.600 \mathrm{mmol}, 2.00$ equiv). The crude material was purified by flash chromatography using MeOH:DCM 1:12 as mobile phase to afford 1r (115 $\mathrm{mg}, 0.226 \mathrm{mmol}, 75 \%$, >20:1 dr) as an off-white solid. TLC $(\mathrm{MeOH}: \mathrm{DCM}, 1: 10 \mathrm{v} / \mathrm{v}): \mathrm{R}_{\mathrm{f}}=$ 0.25, $\mathrm{KMnO}_{4} ;{ }^{1} \mathrm{H} \mathrm{NMR}\left(400 \mathrm{MHz}, \mathrm{CDCl}_{3}\right): \delta 7.59$ (s, $\left.1 \mathrm{H}, \mathrm{ArH}\right), 7.54$ (s, 2H, ArH), 2.66 - 2.57 $(\mathrm{m}, 1 \mathrm{H}), 2.57-2.30(\mathrm{~m}, 7 \mathrm{H}), 2.10-1.92(\mathrm{~m}, 3 \mathrm{H}), 1.88-1.70(\mathrm{~m}, 6 \mathrm{H}), 1.20\left(\mathrm{~s}, 6 \mathrm{H}, \mathrm{OC}\left(\mathrm{CH}_{3}\right)_{2}\right)$, $1.17\left(\mathrm{~s}, 6 \mathrm{H}, \mathrm{OC}\left(\mathrm{CH}_{3}\right)_{2}\right) ;{ }^{13} \mathrm{C} \mathrm{NMR}\left(100 \mathrm{MHz}, \mathrm{CDCl}_{3}\right): \delta 152.5,130.9(\mathrm{q}, J=32.6 \mathrm{~Hz}), 125.9$ (m), 124.1 (q, $J=272.8 \mathrm{~Hz}$ ), 118.5 (hept, $J=3.7 \mathrm{~Hz}$ ), 83.9, 54.2, 43.9, 35.0, 29.1, 25.7, 24.7, 24.6, 23.4; ${ }^{19} \mathrm{~F} \mathrm{NMR}\left(\mathrm{CDCl}_{3}, 376 \mathrm{MHz}\right) \delta$-62.1; IR v 2973 (s), 2935 (m), 2788 (w), 1373 (s), 1276 (s), 1169 (s), 1132 (s), 1065 (m), 892 (m), 850 (m); HRMS (ESI) calcd. for $\mathrm{C}_{24} \mathrm{H}_{33} \mathrm{NO}_{2} \mathrm{BF}_{6}{ }^{+}[\mathrm{M}+\mathrm{H}]^{+}$492.2507; found 492.2515. Carbon attached to boron not observed due to quadrupolar relaxation and one carbon was not resolved at $100 \mathrm{MHz}$. See spectra

\section{1-(2-((1s,2r)-2-(3,5-Bis(trifluoromethyl)phenyl)-2-(4,4,5,5-tetramethyl-1,3,2-}

\section{dioxaborolan-2-yl)cyclobutyl)ethyl)piperidine (1s)}

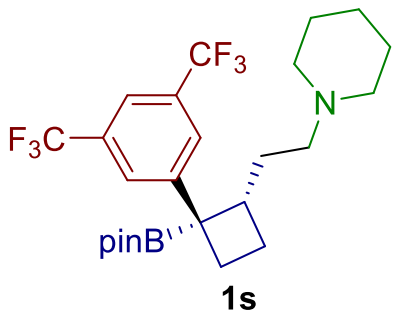

Following general procedure E, using 1-bromo-3,5-bis(trifluoromethyl)benzene (123 mg, $0.420 \mathrm{mmol}, 1.40$ equiv), $n \mathrm{BuLi}(245 \mu \mathrm{L}, 0.390 \mathrm{mmol}, 1.30$ equiv), 4,4,5,5-tetramethyl-2-(1vinylcyclopropyl)-1,3,2-dioxaborolane (4a) (55.3 mg, $0.300 \mathrm{mmol}, 1.00$ equiv), and piperidine derived iminium salt ${ }^{2}$ (135 mg, $0.600 \mathrm{mmol}, 2.00$ equiv). The crude material was purified by flash chromatography using MeOH:DCM 1:10 as mobile phase to afford 1s (116 mg, 0.230 mmol, 77\%, > 20:1 dr) as an off-white solid. TLC (MeOH:DCM, 1:10 v/v): $\mathrm{R}_{\mathrm{f}}=0.24, \mathrm{KMnO}_{4}$; ${ }^{1} \mathrm{H} \mathrm{NMR}\left(400 \mathrm{MHz}, \mathrm{CDCl}_{3}\right): \delta 7.60(\mathrm{~s}, 1 \mathrm{H}, \mathrm{ArH}), 7.52$ (s, 2H, $\left.\mathrm{ArH}\right), 2.63-2.50(\mathrm{~m}, 2 \mathrm{H}), 2.37$ (br s, 4H), $2.32-2.19(\mathrm{~m}, 2 \mathrm{H}), 2.10-1.92(\mathrm{~m}, 3 \mathrm{H}), 1.88-1.70(\mathrm{~m}, 2 \mathrm{H}), 1.59$ (p, J= 5.6 Hz, $4 \mathrm{H}), 1.46-1.41(\mathrm{~m}, 2 \mathrm{H}), 1.21\left(\mathrm{~s}, 6 \mathrm{H}, \mathrm{OC}\left(\mathrm{CH}_{3}\right)_{2}\right), 1.18\left(\mathrm{~s}, 6 \mathrm{H}, \mathrm{OC}\left(\mathrm{CH}_{3}\right)_{2}\right) ;{ }^{13} \mathrm{C} \mathrm{NMR}(100$ $\mathrm{MHz}_{\mathrm{CDCl}}$ ): $\delta 152.4,130.9(\mathrm{q}, J=32.7 \mathrm{~Hz}), 125.8(\mathrm{~m}), 123.7$ (q, $\left.J=272.6 \mathrm{~Hz}\right), 118.5$ (hept, $J=3.6 \mathrm{~Hz}), 83.9,57.2,54.7,44.2,32.7,29.0,26.0,25.7,24.8,24.6,24.5 ;{ }^{19} \mathrm{~F} \mathrm{NMR}\left(\mathrm{CDCl}_{3}\right.$, 
$376 \mathrm{MHz}$ ) $\delta$-62.6; IR v $2977(\mathrm{w}), 2936$ (m), $2860(\mathrm{w}), 1445$ (w), $1373(\mathrm{~m}), 1275(\mathrm{~s}), 1158(\mathrm{~m})$, 1126 (s), 1038 (w), 892 (m), 849 (m); HRMS (ESI) calcd. for $\mathrm{C}_{25} \mathrm{H}_{35} \mathrm{NO}_{2} \mathrm{BF}_{6}{ }^{+}[\mathrm{M}+\mathrm{H}]^{+}$ 506.2660; found 506.2653. Carbon attached to boron not observed due to quadrupolar relaxation. See spectra

\section{2-((1s,2r)-1-(3,5-Bis(trifluoromethyl)phenyl)-2-(cyclohepta-2,4,6-trien-1-} ylmethyl)cyclobutyl)-4,4,5,5-tetramethyl-1,3,2-dioxaborolane (1t)

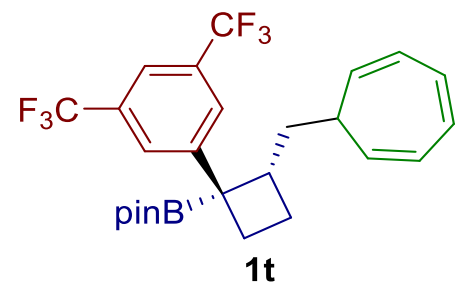

Following general procedure E, using 1-bromo-3,5-bis(trifluoromethyl)benzene (123 mg, 0.420 mmol, 1.40 equiv), $n \mathrm{BuLi}(245 \mu \mathrm{L}, 0.390 \mathrm{mmol}, 1.30$ equiv), 4,4,5,5-tetramethyl-2-(1vinylcyclopropyl)-1,3,2-dioxaborolane (4a) (55.3 mg, $0.300 \mathrm{mmol}, 1.00$ equiv), and tropylium tetrafluoroborate (107 mg, $0.600 \mathrm{mmol}, 2.00$ equiv). The crude material was purified by flash chromatography using DCM:pentane 1:10 as mobile phase to afford $1 \mathrm{t}$ (124 $\mathrm{mg}, 0.249 \mathrm{mmol}$, $83 \%, 20: 1 \mathrm{dr}$ ) as a colorless oil. TLC (DCM:pentane, 1:5 v/v): $\mathrm{R}_{\mathrm{f}}=0.22, \mathrm{KMnO}_{4} ;{ }^{1} \mathrm{H} \mathrm{NMR}$ (400 MHz, $\left.\mathrm{CDCl}_{3}\right): \delta 7.61(\mathrm{~s}, 1 \mathrm{H}, \mathrm{ArH}), 7.48(\mathrm{~s}, 2 \mathrm{H}, \mathrm{Ar} H), 6.74-6.53(\mathrm{~m}, 2 \mathrm{H}$, trop- $H), 6.31$ $-6.01(\mathrm{~m}, 2 \mathrm{H}$, trop- $H), 5.14-5.26(\mathrm{~m}, 2 \mathrm{H}$, trop- $H), 2.81(\mathrm{qd}, J=9.1,6.2 \mathrm{~Hz}, 1 \mathrm{H}), 2.56(\mathrm{t}, J=$ $9.0 \mathrm{~Hz}, 1 \mathrm{H}), 2.24-2.17(\mathrm{~m}, 1 \mathrm{H}), 2.13-1.95(\mathrm{~m}, 3 \mathrm{H}), 1.95-1.83(\mathrm{~m}, 1 \mathrm{H}), 1.72-1.61(\mathrm{~m}$, 1H), 1.21 (s, 6H, OC( $\left.\left.\mathrm{CH}_{3}\right)_{2}\right), 1.19\left(\mathrm{~s}, 6 \mathrm{H}, \mathrm{OC}\left(\mathrm{CH}_{3}\right)_{2}\right) ;{ }^{13} \mathrm{C} \mathrm{NMR}\left(100 \mathrm{MHz}, \mathrm{CDCl}_{3}\right): \delta$ 152.4, 131.0, 130.9 (q, $J=32.7 \mathrm{~Hz}), 126.0,126.0,125.9,125.1,124.9,123.7$ (q, $J=272.6 \mathrm{~Hz}), 118.5$ (hept, $J=3.6 \mathrm{~Hz}), 83.9,43.8,39.1,37.3,29.2,25.9,24.7,24.7 ;{ }^{19} \mathrm{~F} \mathrm{NMR}\left(\mathrm{CDCl}_{3}, 376 \mathrm{MHz}\right)$ $\delta$-62.7; IR v 2979 (w), 1704 (w), 1625 (w), 1470 (w), 1373 (s), 1275 (s), 1167 (s), 1126 (s), 1008 (w), 849 (w); HRMS (ESI) calcd. for $\mathrm{C}_{26} \mathrm{H}_{33} \mathrm{BF}_{6} \mathrm{NO}_{2}{ }^{+}\left[\mathrm{M}+\mathrm{NH}_{4}\right]^{+}$516.2507; found 516.2507. Carbon attached to boron not observed due to quadrupolar relaxation and one carbon was not resolved at $100 \mathrm{MHz}$. See spectra 
2-((1s,2r)-2-(Benzo[d][1,3]dithiol-2-ylmethyl)-1-(3,5-

bis(trifluoromethyl)phenyl)cyclobutyl)-4,4,5,5-tetramethyl-1,3,2-dioxaborolane (1u)

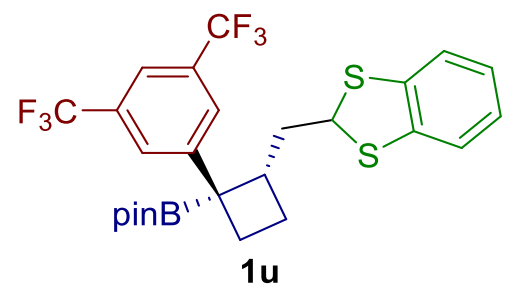

Following general procedure E, using 1-bromo-3,5-bis(trifluoromethyl)benzene (123 mg, 0.420 mmol, 1.40 equiv), $n \operatorname{BuLi}(245 \mu \mathrm{L}, 0.390 \mathrm{mmol}, 1.30$ equiv), 4,4,5,5-tetramethyl-2-(1vinylcyclopropyl)-1,3,2-dioxaborolane (4a) (55.3 $\mathrm{mg}, 0.300 \mathrm{mmol}, 1.00$ equiv), and 1,3benzodithiolylium tetrafluoroborate ( $144 \mathrm{mg}, 0.600 \mathrm{mmol}, 2.00$ equiv). The crude material was purified by flash chromatography using DCM:pentane 1:10 as mobile phase to afford $\mathbf{1 u}(88.0$ $\mathrm{mg}, 0.157 \mathrm{mmol}, 52 \%,>20: 1 \mathrm{dr}$ ) as a colorless oil. TLC (DCM:pentane, 1:5 v/v): $\mathrm{R}_{\mathrm{f}}=0.33$, $\mathrm{KMnO}_{4} ;{ }^{1} \mathrm{H}$ NMR (400 MHz, $\left.\mathrm{CDCl}_{3}\right): \delta 7.62(\mathrm{~s}, 1 \mathrm{H}, \mathrm{ArH}), 7.52(\mathrm{~s}, 2 \mathrm{H}, \mathrm{ArH}), 7.25-7.21(\mathrm{~m}$, 2H, ArH), $7.06-7.01(\mathrm{~m}, 2 \mathrm{H}, \mathrm{ArH}), 4.87$ (dd, $J=7.9,6.3 \mathrm{~Hz}, 1 \mathrm{H}, \mathrm{SCHS}), 2.88$ (qd, $J=9.4$, $4.9 \mathrm{~Hz}, 1 \mathrm{H}), 2.61-2.51(\mathrm{~m}, 1 \mathrm{H}), 2.44-2.37(\mathrm{~m}, 1 \mathrm{H}), 2.30-2.22(\mathrm{~m}, 1 \mathrm{H}), 2.14-2.07(\mathrm{~m}$, 1H), $2.03-1.96(\mathrm{~m}, 1 \mathrm{H}), 1.93-1.83(\mathrm{~m}, 1 \mathrm{H}), 1.21\left(\mathrm{~s}, 6 \mathrm{H}, \mathrm{OC}\left(\mathrm{CH}_{3}\right)_{2}\right), 1.19\left(\mathrm{~s}, 6 \mathrm{H}, \mathrm{OC}\left(\mathrm{CH}_{3}\right)_{2}\right)$; ${ }^{13} \mathrm{C}$ NMR $\left(100 \mathrm{MHz}, \mathrm{CDCl}_{3}\right): \delta 151.5,137.4,136.9,131.1(\mathrm{q}, J=32.9 \mathrm{~Hz}), 125.89-125.73$ (m), 125.5, 123.6 (q, $J=272.6 \mathrm{~Hz}$ ), 122.6, 122.6, 118.8 (hept, $J=3.6 \mathrm{~Hz}$ ), 84.1, 52.6, 44.7, 42.4, 29.7, 25.7, 24.7, 24.6; ${ }^{19} \mathrm{~F} \mathrm{NMR}\left(\mathrm{CDCl}_{3}, 376 \mathrm{MHz}\right) \delta$-62.6; IR v $2978(\mathrm{w}), 2934(\mathrm{w})$, 1616 (w), 1467 (w), 1444 (w), 1374 (m), 1277 (s), 1175 (m), 1134 (s), 849 (w); HRMS (APCI) calcd. for $\mathrm{C}_{26} \mathrm{H}_{28} \mathrm{BF}_{6} \mathrm{O}_{2} \mathrm{~S}_{2}{ }^{+}[\mathrm{M}+\mathrm{H}]^{+}$561.1522; found 561.1492. Carbon attached to boron not observed due to quadrupolar relaxation. See spectra

\section{2-((1s,2r)-1-(3,5-Bis(trifluoromethyl)phenyl)-2-((E)-styryl)cyclobutyl)-4,4,5,5-} tetramethyl-1,3,2-dioxaborolane (1v)

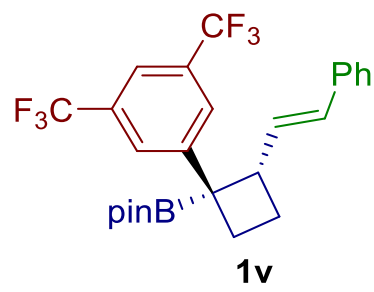

Following general procedure E, using 1-bromo-3,5-bis(trifluoromethyl)benzene (123 mg, 0.420 mmol, 1.40 equiv), $n \mathrm{BuLi}(245 \mu \mathrm{L}, 0.390 \mathrm{mmol}, 1.30$ equiv), 4,4,5,5-tetramethyl-2-(1- 
vinylcyclopropyl)-1,3,2-dioxaborolane (4a) (55.3 mg, $0.300 \mathrm{mmol}, 1.00$ equiv), benzaldehyde dimethyl acetal (69.0 mg, $0.450 \mathrm{mmol}, 1.50$ equiv), and TESOTf (157 mg, $0.450 \mathrm{mmol}, 1.50$ equiv). The crude material was purified by flash chromatography using DCM:pentane 1:10 as mobile phase to afford $1 \mathbf{v}(83.0 \mathrm{mg}, 0.167 \mathrm{mmol}, 56 \%, 14: 1 \mathrm{dr})$ as a colorless oil. TLC DCM:pentane, 1:5 v/v): $\mathrm{R}_{\mathrm{f}}=0.21, \mathrm{KMnO}_{4} ;{ }^{1} \mathrm{H} \mathrm{NMR}\left(400 \mathrm{MHz}, \mathrm{CDCl}_{3}\right): \delta 7.64(\mathrm{~s}, 1 \mathrm{H}, \mathrm{Ar} H)$, 7.58 (s, 2H, ArH), $7.43-7.38$ (m, 2H, ArH), $7.35-7.29$ (m, 2H, ArH), $7.26-7.20(\mathrm{~m}, 1 \mathrm{H}$, $\operatorname{Ar} H), 6.62(\mathrm{dd}, J=15.8,7.7 \mathrm{~Hz}, 1 \mathrm{H}, \mathrm{CH}=\mathrm{CH}), 6.44(\mathrm{~d}, J=15.8 \mathrm{~Hz}, 1 \mathrm{H}, \mathrm{CH}=\mathrm{CH}), 3.32-3.23$ $(\mathrm{m}, 1 \mathrm{H}), 2.62-2.46(\mathrm{~m}, 1 \mathrm{H}), 2.37-2.25(\mathrm{~m}, 1 \mathrm{H}), 2.22-2.03(\mathrm{~m}, 2 \mathrm{H}), 1.18\left(\mathrm{~s}, 6 \mathrm{H}, \mathrm{OC}\left(\mathrm{CH}_{3}\right)_{2}\right)$, $1.17\left(\mathrm{~s}, 6 \mathrm{H}, \mathrm{OC}\left(\mathrm{CH}_{3}\right)_{2}\right) ;{ }^{13} \mathrm{C} \mathrm{NMR}\left(100 \mathrm{MHz}, \mathrm{CDCl}_{3}\right): \delta 151.9,137.4,132.6,131.0(\mathrm{q}, J=32.8$ $\mathrm{Hz}), 129.7,128.6,127.2,126.2,125.8-125.6(\mathrm{~m}), 123.6$ (q, $J=272.6 \mathrm{~Hz}), 118.7$ (hept, $J=$ 3.6 Hz), 84.0, 49.4, 28.2, 25.0, 24.8, 24.6; ${ }^{19} \mathrm{~F} \mathrm{NMR}\left(\mathrm{CDCl}_{3}, 376 \mathrm{MHz}\right) \delta$-62.7; IR v $2925(\mathrm{w})$, 1701 (m), 1619 (w), 1382 (m), 1275 (s), 1128 (s), 901 (m), 843 (w); HRMS (APCI) calcd. for $\mathrm{C}_{26} \mathrm{H}_{28} \mathrm{BF}_{6} \mathrm{O}_{2}{ }^{+}[\mathrm{M}+\mathrm{H}]^{+}$497.2081; found 497.2075. Carbon attached to boron not observed due to quadrupolar relaxation. See spectra

\section{2-((1s,2s)-1-(3,5-Bis(trifluoromethyl)phenyl)-2-((methylthio)methyl)cyclobutyl)-4,4,5,5- tetramethyl-1,3,2-dioxaborolane (1w)}

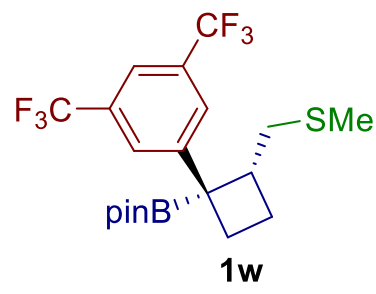

Following general procedure E, using 1-bromo-3,5-bis(trifluoromethyl)benzene (123 mg, 0.420 mmol, 1.40 equiv), $n \mathrm{BuLi}(245 \mu \mathrm{L}, 0.390 \mathrm{mmol}, 1.30$ equiv), 4,4,5,5-tetramethyl-2-(1vinylcyclopropyl)-1,3,2-dioxaborolane (4a) $(55.3 \mathrm{mg}, 0.300 \mathrm{mmol}, 1.00$ equiv), and dimethyl(methylthio)sulfonium tetrafluoroborate (118 mg, $0.600 \mathrm{mmol}, 2.00$ equiv). The crude material was purified by flash chromatography using DCM:pentane 1:10 as mobile phase to afford $1 \mathbf{w}(83.0 \mathrm{mg}, 0.183 \mathrm{mmol}, 61 \%$, >20:1 dr) as a colorless oil. TLC (DCM:pentane, 1:5 $\mathrm{v} / \mathrm{v}): \mathrm{R}_{\mathrm{f}}=0.32, \mathrm{KMnO}_{4} ;{ }^{1} \mathrm{H} \mathrm{NMR}\left(400 \mathrm{MHz}, \mathrm{CDCl}_{3}\right): \delta 7.62(\mathrm{~s}, 3 \mathrm{H}, \mathrm{ArH}), 3.07-2.88(\mathrm{~m}$, $1 \mathrm{H}), 2.83-2.65(\mathrm{~m}, 2 \mathrm{H}), 2.61-2.41(\mathrm{~m}, 1 \mathrm{H}), 2.21-2.13(\mathrm{~m}, 1 \mathrm{H}), 2.12\left(\mathrm{~s}, 3 \mathrm{H}, \mathrm{SCH}_{3}\right), 2.12-$ $2.02(\mathrm{~m}, 1 \mathrm{H}), 2.01-1.83(\mathrm{~m}, 1 \mathrm{H}), 1.21\left(\mathrm{~s}, 6 \mathrm{H}, \mathrm{OC}\left(\mathrm{CH}_{3}\right)_{2}\right), 1.19\left(\mathrm{~s}, 6 \mathrm{H}, \mathrm{OC}\left(\mathrm{CH}_{3}\right)_{2}\right) ;{ }^{13} \mathrm{C} \mathrm{NMR}$ $\left(100 \mathrm{MHz}, \mathrm{CDCl}_{3}\right): \delta 151.5,131.0(\mathrm{q}, J=32.8 \mathrm{~Hz}), 126.2-126.1(\mathrm{~m}), 123.6(\mathrm{q}, J=272.7 \mathrm{~Hz})$, 118.7 (hept, $J=3.6 \mathrm{~Hz}), 84.0,45.1,38.9,28.3,25.6,24.7,24.6,15.7 ;{ }^{19} \mathrm{~F} \mathrm{NMR}\left(\mathrm{CDCl}_{3}, 376\right.$ 
MHz) $\delta$-62.8; IR v 2978 (m), 1616 (w), 1511445 (w), 1373 (s), 1276 (s), 1168 (s), 1131 (s), 955 (w); HRMS (APCI) calcd. for $\mathrm{C}_{20} \mathrm{H}_{26} \mathrm{BF}_{6} \mathrm{O}_{2} \mathrm{~S}^{+}[\mathrm{M}+\mathrm{H}]^{+}$455.1573; found 454.1579. Carbon attached to boron not observed due to quadrupolar relaxation. See spectra

\section{2-((1r,2r)-1-(3,5-Bis(trifluoromethyl)phenyl)-2-(fluoromethyl)cyclobutyl)-4,4,5,5-} tetramethyl-1,3,2-dioxaborolane (1x)

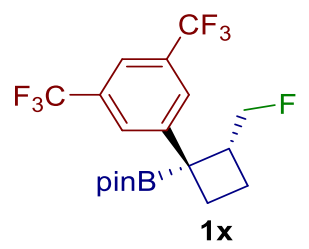

To a stirred solution of 1-bromo-3,5-bis(trifluoromethyl)benzene (123 mg, $0.420 \mathrm{mmol}, 1.40$ equiv) in THF ( $1 \mathrm{~mL})$ under $\mathrm{N}_{2}$ at $-78^{\circ} \mathrm{C}$ was added $n \mathrm{BuLi}(0.390 \mathrm{mmol}, 1.30$ equiv) dropwise. The solution was then stirred for $1 \mathrm{~h}$ at $-78{ }^{\circ} \mathrm{C}$, at which point a solution of 4,4,5,5-tetramethyl2-(1-vinylcyclopropyl)-1,3,2-dioxaborolane (4a) (55.3 mg, $0.300 \mathrm{mmol}, 1.00$ equiv) in THF (1 $\mathrm{mL}$ ) was added dropwise. The reaction mixture was stirred at $-78^{\circ} \mathrm{C}$ for $1 \mathrm{~h}$ and then warmed to RT and stirred for another $1 \mathrm{~h}$. The reaction mixture was cooled back to $-78{ }^{\circ} \mathrm{C}$ and $\mathrm{CH}_{3} \mathrm{CN}$ $(0.2 \mathrm{~mL})$ was added and the reaction mixture was warmed up to $0{ }^{\circ} \mathrm{C}$ and stirred for $5 \mathrm{~min}$. The volatiles were then removed in vacuo (using a Schlenk line) at $0{ }^{\circ} \mathrm{C}$ followed by re-establishing $\mathrm{N}_{2}$ atmosphere and the addition of $\mathrm{CH}_{3} \mathrm{CN}(2 \mathrm{~mL})$. In a separate flask, to a stirred suspension of Selectfluor II (213 mg, $0.600 \mathrm{mmol}, 2.00$ equiv) in $\mathrm{CH}_{3} \mathrm{CN}$ (3 mL), under $\mathrm{N}_{2}$ at $-40{ }^{\circ} \mathrm{C}$, was added the pre-prepared solution of ate complex in $\mathrm{CH}_{3} \mathrm{CN}$ dropwise by syringe. The reaction mixture was stirred at $-40{ }^{\circ} \mathrm{C}$ for $16 \mathrm{~h}$. The reaction was quenched by addition of sat. aq. $\mathrm{NaHCO} 3$ $(15 \mathrm{~mL})$ and allowed to warm to RT. The aqueous phase was extracted with $\mathrm{Et}_{2} \mathrm{O}(3 \times 15 \mathrm{~mL})$, the organic layers were combined, washed with brine, dried over $\mathrm{MgSO}_{4}$, filtered and concentrated in vасио. The crude material was purified by flash chromatography using DCM:pentane 1:10 as mobile phase to afford $1 \times(32.0 \mathrm{mg}, 0.075 \mathrm{mmol}, 25 \%,>20: 1 \mathrm{dr})$ as a colorless oil. TLC (DCM:pentane, 1:5 v/v): $\mathrm{R}_{\mathrm{f}}=0.21, \mathrm{KMnO}_{4} ;{ }^{1} \mathrm{H} \mathrm{NMR}\left(400 \mathrm{MHz}, \mathrm{CDCl}_{3}\right): \delta 7.63(\mathrm{~s}, 1 \mathrm{H}, \mathrm{ArH})$, $7.57(\mathrm{~s}, 2 \mathrm{H}, \mathrm{Ar} H), 4.77(\mathrm{dd}, J=9.6,7.2 \mathrm{~Hz}, 0.5 \mathrm{H}), 4.72-4.63(\mathrm{~m}, 1 \mathrm{H}), 4.57$ (dd, $J=9.5,5.3$ $\mathrm{Hz}, 0.5 \mathrm{H}), 2.99-2.83(\mathrm{~m}, 1 \mathrm{H}), 2.63-2.55(\mathrm{~m}, 1 \mathrm{H}), 2.21-2.13(\mathrm{~m}, 1 \mathrm{H}), 2.08-1.95(\mathrm{~m}, 2 \mathrm{H})$, $1.19\left(\mathrm{~s}, 6 \mathrm{H}, \mathrm{OC}\left(\mathrm{CH}_{3}\right)_{2}\right), 1.17$ (s, $\left.6 \mathrm{H}, \mathrm{OC}\left(\mathrm{CH}_{3}\right)_{2}\right) ;{ }^{13} \mathrm{C} \mathrm{NMR}\left(100 \mathrm{MHz}, \mathrm{CDCl}_{3}\right): \delta 151.3,131.0$ (q, $J=32.8 \mathrm{~Hz}), 126.1-126.0(\mathrm{~m}), 123.6(\mathrm{q}, J=272.5 \mathrm{~Hz}), 118.8$ (hept, $J=3.6 \mathrm{~Hz}), 85.3$ (d, $J=166.2 \mathrm{~Hz}), 84.1,45.2(\mathrm{~d}, J=18.8 \mathrm{~Hz}), 28.6,24.5,24.5,20.5(\mathrm{~d}, J=9.3 \mathrm{~Hz}) ;{ }^{19} \mathrm{~F}$ NMR $\left(\mathrm{CDCl}_{3}, 376 \mathrm{MHz}\right) \delta-62.6,-216.6(\mathrm{td}, J=47.5,18.8 \mathrm{~Hz}) ; \mathrm{IR} v 2980(\mathrm{w}), 1617(\mathrm{w}), 1457(\mathrm{w})$, 
1374 (s), 1322 (m), 1276 (s), 1169 (m), 1130 (s), 894 (w). Carbon attached to boron not observed due to quadrupolar relaxation. See spectra

\section{2-((1s,2s)-1-(3,5-Bis(trifluoromethyl)phenyl)-2-methylcyclobutyl)-4,4,5,5-tetramethyl-}

\section{1,3,2-dioxaborolane $(1 \mathrm{y})$}

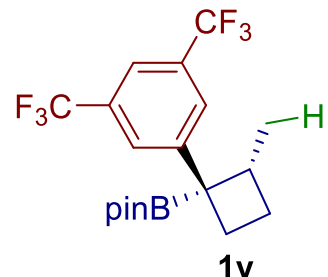

$1 y$

Following general procedure E, using 1-bromo-3,5-bis(trifluoromethyl)benzene (123 mg, 0.420 mmol, 1.40 equiv), $n \mathrm{BuLi}(245 \mu \mathrm{L}, 0.390 \mathrm{mmol}, 1.30$ equiv), 4,4,5,5-tetramethyl-2-(1vinylcyclopropyl)-1,3,2-dioxaborolane (4a) (55.3 $\mathrm{mg}, 0.300 \mathrm{mmol}, 1.00$ equiv), and $\mathrm{HBF}_{4} . \mathrm{OEt}_{2}(98.0 \mathrm{mg}, 83 \mu \mathrm{L}, 0.600 \mathrm{mmol}, 2.00$ equiv). The aqueous layer was extracted with pentane instead of $\mathrm{Et}_{2} \mathrm{O}$. The crude material was purified by flash chromatography using DCM:pentane 1:10 as mobile phase to afford 1y (105 mg, $0.257 \mathrm{mmol}, 86 \%,>20: 1 \mathrm{dr}$ ) as a colorless oil. TLC (DCM:pentane, 1:5 v/v): $\mathrm{R}_{\mathrm{f}}=0.18, \mathrm{KMnO}_{4} ;{ }^{1} \mathrm{H} \mathrm{NMR}\left(400 \mathrm{MHz}, \mathrm{CDCl}_{3}\right)$ : $\delta 7.61(\mathrm{~s}, 1 \mathrm{H}, \operatorname{Ar} H), 7.51$ (s, 2H, ArH), $2.67-2.59$ (m, 1H), $2.56-2.49$ (m, 1H), $2.13-1.94$ $(\mathrm{m}, 2 \mathrm{H}), 1.88-1.75(\mathrm{~m}, 1 \mathrm{H}), 1.32\left(\mathrm{~d}, J=7.0 \mathrm{~Hz}, 3 \mathrm{H}, \mathrm{CHCH}_{3}\right), 1.22\left(\mathrm{~s}, 6 \mathrm{H}, \mathrm{OC}\left(\mathrm{CH}_{3}\right)_{2}\right), 1.19$ $\left(\mathrm{s}, 6 \mathrm{H}, \mathrm{OC}\left(\mathrm{CH}_{3}\right)_{2}\right) ;{ }^{13} \mathrm{C} \mathrm{NMR}\left(100 \mathrm{MHz}, \mathrm{CDCl}_{3}\right): \delta 152.6,130.9(\mathrm{q}, J=32.7 \mathrm{~Hz}), 125.6-125.7$ (m), 123.5 (q, $J=272.7 \mathrm{~Hz}), 118.5$ (hept, $J=4.0 \mathrm{~Hz}$ ), 83.9, 41.5, 28.5, 27.2, 24.8, 24.6, 20.4; ${ }^{19} \mathrm{~F} \mathrm{NMR}\left(\mathrm{CDCl}_{3}, 376 \mathrm{MHz}\right) \delta$-62.6; IR v 2979 (m), 2339 (w), 1703 (w), 1474 (m), 1374 (m), 1277 (s), 1173 (s), 1135 (s), 981 (w); $\mathrm{HRMS}$ (APCI) calcd. for $\mathrm{C}_{19} \mathrm{H}_{24} \mathrm{BF}_{6} \mathrm{O}_{2}{ }^{+}[\mathrm{M}+\mathrm{H}]^{+}$ 409.1768; found 409.1754. Carbon attached to boron not observed due to quadrupolar relaxation. See spectra

$N, N$-Dimethyl-2-((1r,2s)-2-phenyl-2-(4,4,5,5-tetramethyl-1,3,2-dioxaborolan-2-yl)cyclob utyl)propan-1-amine (1z)

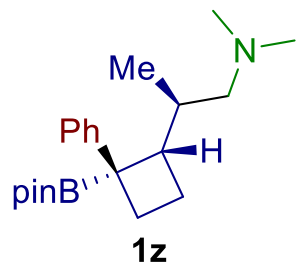


Following general procedure $\mathbf{F}$, using (E)-4,4,5,5-tetramethyl-2-(1-(prop-1-en-1yl)cyclopropyl)-1,3,2-dioxaborolane (4b) $(63.0 \mathrm{mg}, 0.300 \mathrm{mmol}, 1.00$ equiv), PhLi (206 $\mu \mathrm{L}$, $0.390 \mathrm{mmol}, 1.90 \mathrm{M}$ in dibutyl ether, 1.30 equiv), and Eschenmoser's salt (5) (111 mg, 0.600 mmol, 2.00 equiv). The crude material was purified by a quick flash chromatography using MeOH:DCM 1:20 as mobile phase to afford 1z $(59.0 \mathrm{mg}, 0.171 \mathrm{mmol}, 57 \%,>20: 1 \mathrm{dr})$ as a white solid. Mp: $57.0-62.0{ }^{\circ} \mathrm{C}$; TLC (MeOH:DCM, 1:10 v/v): $\mathrm{R}_{\mathrm{f}}=0.22, \mathrm{KMnO}_{4} ;{ }^{1} \mathrm{H} \mathrm{NMR}$ (400 MHz, $\left.\mathrm{CDCl}_{3}\right): 7.28$ - $7.21(\mathrm{~m}, 2 \mathrm{H}, \mathrm{ArH}), 7.15$ - 7.06 (m, 3H, $\left.\mathrm{ArH}\right), 2.54-2.38(\mathrm{~m}, 2 \mathrm{H})$, $2.45\left(\mathrm{~s}, 6 \mathrm{H},\left(\mathrm{CH}_{3}\right)_{2} \mathrm{~N}\right), 2.37-2.31\left(\mathrm{~m}, 2 \mathrm{H}, \mathrm{CH}_{2}\left(\mathrm{CH}_{3}\right)_{2} \mathrm{~N}\right), 2.10-1.96(\mathrm{~m}, 2 \mathrm{H}), 1.94-1.75(\mathrm{~m}$, $2 \mathrm{H}), 1.23\left(\mathrm{~s}, 6 \mathrm{H}, \mathrm{OC}\left(\mathrm{CH}_{3}\right)_{2}\right), 1.21\left(\mathrm{~s}, 6 \mathrm{H}, \mathrm{OC}\left(\mathrm{CH}_{3}\right)_{2}\right), 1.19\left(\mathrm{~d}, \mathrm{~J}=6.5 \mathrm{~Hz}, 3 \mathrm{H}, \mathrm{CHCH}_{3}\right) ;{ }^{13} \mathrm{C}$ NMR (100 MHz, $\left.\mathrm{CDCl}_{3}\right): \delta 149.5,128.0,126.2,124.8,83.6,63.7,48.1,45.2,36.8,30.5,24.9$, 24.8, 24.7, 17.8; IR $v 2974$ (m), 1462 (w), 1344 (m), 1309 (m), 1142 (s), 846 (m), 699 (s); HRMS (ESI) calcd. for $\mathrm{C}_{21} \mathrm{H}_{35} \mathrm{BNO}_{2}{ }^{+}[\mathrm{M}+\mathrm{H}]^{+}$344.2755; found: 344.2743. Carbon attached to boron not observed due to quadrupolar relaxation. $\underline{\text { See spectra }}$

(r)- $N, N$-Dimethyl-2-((1s,2r)-2-phenyl-2-(4,4,5,5-tetramethyl-1,3,2-dioxaborolan-2yl)cyclobutyl)hexan-1-amine (1aa)

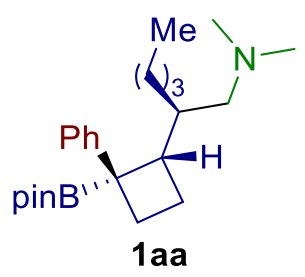

Following general procedure E, using (E)-2-(1-(Hex-1-en-1-yl)cyclopropyl)-4,4,5,5tetramethyl-1,3,2-dioxaborolane (4c) (75.0 mg, 0.300 mmol, 1.00 equiv), PhLi (206 $\mu \mathrm{L}, 0.390$ mmol, 1.90 $\mathrm{M}$ in dibutyl ether, 1.30 equiv), and Eschenmoser's salt (5) (111 mg, $0.600 \mathrm{mmol}$, 2.00 equiv). The crude material was purified by a quick flash chromatography using MeOH:DCM 1:15 as mobile phase to afford 1aa (96.5 mg, $0.250 \mathrm{mmol}, 84 \%,>20: 1 \mathrm{dr}$ ) as a colorless liquid. TLC (MeOH:DCM, 1:10 v/v): $\mathrm{R}_{\mathrm{f}}=0.26, \mathrm{KMnO}_{4} ;{ }^{1} \mathrm{H}$ NMR $(400 \mathrm{MHz}$, $\left.\mathrm{CDCl}_{3}\right): \delta 7.27-7.20(\mathrm{~m}, 2 \mathrm{H}, \mathrm{ArH}), 7.20-7.15$ (m, 2H, $\left.\mathrm{ArH}\right), 7.11-7.06$ (m, 1H), 2.59 (q, $J$ $=10.0,9.4 \mathrm{~Hz}, 1 \mathrm{H}), 2.48-2.38(\mathrm{~m}, 1 \mathrm{H}), 2.20\left(\mathrm{~s}, 6 \mathrm{H},\left(\mathrm{CH}_{3}\right)_{2} \mathrm{~N}\right), 2.07-2.00(\mathrm{~m}, 1 \mathrm{H}), 1.99-$ $1.93(\mathrm{~m}, 1 \mathrm{H}), 1.90-1.80(\mathrm{~m}, 3 \mathrm{H}), 1.61-1.49(\mathrm{~m}, 1 \mathrm{H}), 1.39-1.12(\mathrm{~m}, 18 \mathrm{H}), 0.84(\mathrm{t}, J=6.9$ $\left.\mathrm{Hz}, 3 \mathrm{H}, \mathrm{CH}_{2} \mathrm{CH}_{3}\right) ;{ }^{13} \mathrm{C} \mathrm{NMR}\left(100 \mathrm{MHz}, \mathrm{CDCl}_{3}\right): \delta 149.9,127.8,126.3,124.4,83.1,62.1,47.6$, 46.4, 41.3, 30.8, 30.7, 28.4, 25.1, 24.7, 24.6, 23.4, 14.3; IR $v 2954$ (m), 2931 (s), 2857 (w), 1443 (m), 1378 (m), 1307 (s), 1143 (s), 1109 (m), 873 (w), 846 (s); HRMS (ESI) calcd. for 
$\mathrm{C}_{24} \mathrm{H}_{41} \mathrm{BNO}_{2}{ }^{+}[\mathrm{M}+\mathrm{H}]^{+}$386.3225; found 386.3218. Carbon attached to boron not observed due to quadrupolar relaxation. See spectra

(r)-N,N-Dimethyl-4-phenyl-2-((1s,2r)-2-phenyl-2-(4,4,5,5-tetramethyl-1,3,2dioxaborolan-2-yl)cyclobutyl)butan-1-amine (1ab)

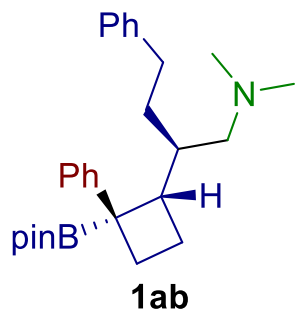

Following general procedure F, using (E)-4,4,5,5-tetramethyl-2-(1-(4-phenylbut-1-en-1yl)cyclopropyl)-1,3,2-dioxaborolane (4e) (89.5 mg, $0.300 \mathrm{mmol}, 1.00$ equiv), PhLi (206 $\mu \mathrm{L}$, $0.390 \mathrm{mmol}, 1.90 \mathrm{M}$ in dibutyl ether, 1.30 equiv), and Eschenmoser's salt (5) (111 mg, 0.600 mmol, 2.00 equiv). The crude material was purified by a quick flash chromatography using MeOH:DCM 1:12 as mobile phase to afford 1ab $(70.0 \mathrm{mg}, 0.161 \mathrm{mmol}, 54 \%,>20: 1 \mathrm{dr})$ as a white solid. Mp: $87.0-92.5{ }^{\circ} \mathrm{C}$; TLC (MeOH:DCM, 1:10 v/v): $\mathrm{R}_{\mathrm{f}}=0.28, \mathrm{KMnO}_{4} ;{ }^{1} \mathrm{H}$ NMR $\left(400 \mathrm{MHz}, \mathrm{CDCl}_{3}\right): \delta 7.25$ - $7.19(\mathrm{~m}, 6 \mathrm{H}, \mathrm{ArH}), 7.15-7.07(\mathrm{~m}, 4 \mathrm{H}, \mathrm{ArH}), 2.72-2.62(\mathrm{~m}$, $2 \mathrm{H}), 2.61-2.52(\mathrm{~m}, 1 \mathrm{H}), 2.51-2.39(\mathrm{~m}, 1 \mathrm{H}), 2.33-2.28(\mathrm{~m}, 1 \mathrm{H}), 2.32-2.21(\mathrm{~m}, 7 \mathrm{H}), 2.14$ $(\mathrm{dd}, \mathrm{J}=12.3,4.2 \mathrm{~Hz}, 1 \mathrm{H}), 2.06-1.95(\mathrm{~m}, 2 \mathrm{H}), 1.95-1.81(\mathrm{~m}, 2 \mathrm{H}), 1.63(\mathrm{tdd}, \mathrm{J}=12.2,6.6$, $4.8 \mathrm{~Hz}, 1 \mathrm{H}), 1.26\left(\mathrm{~s}, 6 \mathrm{H}, \mathrm{OC}\left(\mathrm{CH}_{3}\right)_{2}\right), 1.25\left(\mathrm{~s}, 6 \mathrm{H}, \mathrm{OC}\left(\mathrm{CH}_{3}\right)_{2}\right) ;{ }^{13} \mathrm{C} \mathrm{NMR}\left(100 \mathrm{MHz}, \mathrm{CDCl}_{3}\right): \delta$ 149.5, 143.6, 128.6, 128.2, 128.1, 126.6, 125.5, 124.8, 83.4, 62.2, 48.0, 46.4, 41.5, 33.4, 32.7, 30.8, 25.2, 24.9, 24.8; IR v 2974 (m), 2935 (m), 1308 (m), 1143 (s), 1032 (w), 847 (w), 698 (s); HRMS (ESI) calcd. for $\mathrm{C}_{28} \mathrm{H}_{41} \mathrm{BNO}_{2}{ }^{+}[\mathrm{M}+\mathrm{H}]^{+}$434.3229; found: 434.3223. Carbon attached to boron not observed due to quadrupolar relaxation. $\underline{\text { See spectra }}$

(s)-4-(Dimethylamino)-3-((1s,2r)-2-phenyl-2-(4,4,5,5-tetramethyl-1,3,2-dioxaborolan-2yl)cyclobutyl)butan-1-ole (1ac)

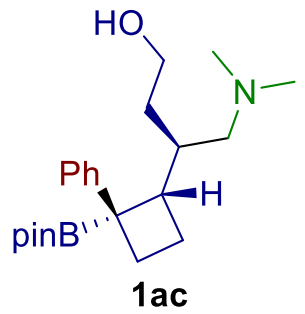


Following general procedure $\mathbf{E}$, using $(E)$-tert-butyldimethyl((4-(1-(4,4,5,5-tetramethyl-1,3,2dioxaborolan-2-yl)cyclopropyl)but-3-en-1-yl)oxy)silane (4d) (106 mg, 0.300 mmol, 1.00 equiv), PhLi (206 $\mu \mathrm{L}, 0.390 \mathrm{mmol}, 1.90 \mathrm{M}$ in dibutyl ether, 1.30 equiv), and Eschenmoser's salt (5) (111 mg, $0.600 \mathrm{mmol}, 2.00$ equiv). The crude material was purified by a quick flash chromatography using MeOH:DCM 1:10 as mobile phase to afford 1ac (103 mg, $0.276 \mathrm{mmol}$, $92 \%,>20: 1 \mathrm{dr})$ as a white amorphous solid. TLC $(\mathrm{MeOH}: \mathrm{DCM}, 1: 10 \mathrm{v} / \mathrm{v}): \mathrm{R}_{\mathrm{f}}=0.22, \mathrm{KMnO}_{4}$; ${ }^{1} \mathrm{H}$ NMR (400 MHz, $\mathrm{CDCl}_{3}$ ): $\delta 7.28-7.22$ (m, 2H, $\left.\mathrm{ArH}\right), 7.17-7.06$ (m, 3H, ArH), 3.68 (ddd, $J=11.6,4.7,2.7 \mathrm{~Hz}, 1 \mathrm{H}), 3.49-3.43(\mathrm{~m}, 1 \mathrm{H}), 2.60-2.40(\mathrm{~m}, 2 \mathrm{H}), 2.27\left(\mathrm{~s}, 6 \mathrm{H},\left(\mathrm{CH}_{3}\right)_{2} \mathrm{~N}\right)$, $2.25-2.14(\mathrm{~m}, 1 \mathrm{H}), 2.11-1.97(\mathrm{~m}, 3 \mathrm{H}), 1.94-1.82(\mathrm{~m}, 1 \mathrm{H}), 1.82-1.70(\mathrm{~m}, 2 \mathrm{H}), 1.41-1.27$ $(\mathrm{m}, 1 \mathrm{H}), 1.25\left(\mathrm{~s}, 6 \mathrm{H}, \mathrm{OC}\left(\mathrm{CH}_{3}\right)_{2}\right), 1.24\left(\mathrm{~s}, 6 \mathrm{H}, \mathrm{OC}\left(\mathrm{CH}_{3}\right)_{2}\right) ;{ }^{13} \mathrm{C} \mathrm{NMR}\left(100 \mathrm{MHz}, \mathrm{CDCl}_{3}\right): \delta$ 149.2, 128.0, 126.1, 124.7, 83.4, 63.2, 62.2, 46.2, 45.2, 44.6, 37.9, 30.8, 25.4, 25.1, 24.6; IR v 2975 (s), 1464 (m), 1443 (m), 1379 (m), 1308 (s), 1237 (m), 1142 (s), 1076 (s), 1024 (m), 846 (s); HRMS (ESI) calcd. for $\mathrm{C}_{22} \mathrm{H}_{37} \mathrm{BNO}_{3}{ }^{+}[\mathrm{M}+\mathrm{H}]^{+}$374.2865; found 374.2860. Carbon attached to boron not observed due to quadrupolar relaxation. $\underline{\text { See spectra }}$

\section{(r)-2-Cyclopropyl- $N, N$-dimethyl-2-((1s,2r)-2-phenyl-2-(4,4,5,5-tetramethyl-1,3,2-}

\section{dioxaborolan-2-yl)cyclobutyl)ethan-1-amine (1ad)}

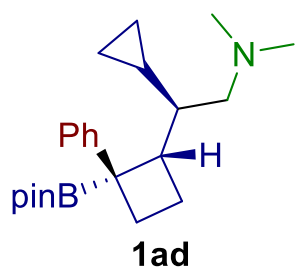

Following general procedure F, using (E)-2-(1-(2-cyclopropylvinyl)cyclopropyl)-4,4,5,5tetramethyl-1,3,2-dioxaborolane (4f) (70.5 mg, $0.300 \mathrm{mmol}, 1.00$ equiv), $\mathrm{PhLi}$ (206 $\mu \mathrm{L}, 0.390$ mmol, 1.90 $\mathrm{M}$ in dibutyl ether, 1.30 equiv), and Eschenmoser's salt (5) (111 mg, $0.600 \mathrm{mmol}$, 2.00 equiv). The crude material was purified by a quick flash chromatography using MeOH:DCM 1:15 as mobile phase to afford 1ad (89.5 mg, $0.242 \mathrm{mmol}, 81 \%,>20: 1 \mathrm{dr}$ ) as a colorless liquid. TLC (MeOH:DCM, 1:10 v/v): $\mathrm{R}_{\mathrm{f}}=0.24, \mathrm{KMnO}_{4} ;{ }^{1} \mathrm{H}$ NMR $(400 \mathrm{MHz}$, $\left.\mathrm{CDCl}_{3}\right): \delta 7.33-7.28(\mathrm{~m}, 2 \mathrm{H}, \mathrm{ArH}), 7.26-7.20(\mathrm{~m}, 2 \mathrm{H}, \mathrm{ArH}), 7.11-7.05(\mathrm{~m}, 1 \mathrm{H}, \mathrm{ArH}), 2.68$ $(\mathrm{td}, J=10.3,8.5 \mathrm{~Hz}, 1 \mathrm{H}), 2.48-2.37(\mathrm{~m}, 1 \mathrm{H}), 2.20\left(\mathrm{~s}, 6 \mathrm{H},\left(\mathrm{CH}_{3}\right)_{2} \mathrm{~N}\right), 2.15-2.05(\mathrm{~m}, 2 \mathrm{H})$, $2.04-1.91(\mathrm{~m}, 2 \mathrm{H}), 1.88-1.81(\mathrm{~m}, 1 \mathrm{H}), 1.64-1.57(\mathrm{~m}, 1 \mathrm{H}), 1.24\left(\mathrm{~s}, 6 \mathrm{H}, \mathrm{OC}\left(\mathrm{CH}_{3}\right)_{2}\right), 1.23$ $\left(\mathrm{s}, 6 \mathrm{H}, \mathrm{OC}\left(\mathrm{CH}_{3}\right)_{2}\right), 0.71-0.80(\mathrm{~m}, 1 \mathrm{H}), 0.55-0.39(\mathrm{~m}, 2 \mathrm{H}), 0.33(\mathrm{tdd}, J=8.7,5.6,4.1 \mathrm{~Hz}$, $1 \mathrm{H}), 0.19(\mathrm{dtd}, J=9.4,5.4,4.0 \mathrm{~Hz}, 1 \mathrm{H}) ;{ }^{13} \mathrm{C} \mathrm{NMR}\left(100 \mathrm{MHz}, \mathrm{CDCl}_{3}\right): \delta 150.1,127.6,126.5$, 124.3, 83.1, 62.0, 48.4, 46.4, 43.1, 31.5, 25.1, 24.8, 24.4, 14.3, 3.9, 2.9; IR v 3075 (w), 2974 
(s), 2940 (s), 1459 (s), 1370 (s), 1143 (s), 1020 (m), 848 (w); HRMS (ESI) calcd. for $\mathrm{C}_{23} \mathrm{H}_{37} \mathrm{BNO}_{2}{ }^{+}[\mathrm{M}+\mathrm{H}]^{+} 370.2916$; found 370.2918. Carbon attached to boron not observed due to quadrupolar relaxation. See spectra

(s)- $N, N$-dimethyl-2-phenyl-2-((1s,2r)-2-phenyl-2-(4,4,5,5-tetramethyl-1,3,2dioxaborolan-2-yl)cyclobutyl)ethan-1-amine (1ae)

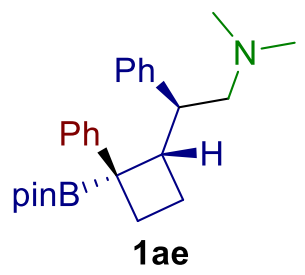

Following general procedure F, using (E)-4,4,5,5-tetramethyl-2-(1-styrylcyclopropyl)-1,3,2dioxaborolane (4g) (81.0 mg, $0.300 \mathrm{mmol}, 1.00$ equiv), PhLi (206 $\mu \mathrm{L}, 0.390 \mathrm{mmol}, 1.90 \mathrm{M}$ in dibutyl ether, 1.30 equiv), and Eschenmoser's salt (5) (111 mg, $0.600 \mathrm{mmol}, 2.00$ equiv). The crude material was purified by a quick flash chromatography using MeOH:DCM 1:12 as mobile phase to afford 1ae $(66.0 \mathrm{mg}, 0.163 \mathrm{mmol}, 54 \%,>20: 1 \mathrm{dr})$ as a white solid. Mp: 98.5 $103{ }^{\circ} \mathrm{C} ; \mathrm{TLC}(\mathrm{MeOH}: \mathrm{DCM}, 1: 10 \mathrm{v} / \mathrm{v}): \mathrm{R}_{\mathrm{f}}=0.22, \mathrm{KMnO}_{4} ;{ }^{1} \mathrm{H} \mathrm{NMR}\left(400 \mathrm{MHz}, \mathrm{CDCl}_{3}\right): \delta 7.26$ -7.17 (m, 5H, ArH), $6.91-6.85$ (m, 3H, ArH), $6.30-6.24$ (m, 2H, ArH), 3.48 (td, $J=10.8$, $3.7 \mathrm{~Hz}, 1 \mathrm{H}), 2.66(\mathrm{t}, \mathrm{J}=11.6 \mathrm{~Hz}, 1 \mathrm{H}), 2.54(\mathrm{td}, J=10.3,7.6 \mathrm{~Hz}, 1 \mathrm{H}), 2.49-2.40(\mathrm{~m}, 1 \mathrm{H}), 2.34$ $-2.25(\mathrm{~m}, 1 \mathrm{H}), 2.19\left(\mathrm{~s}, 6 \mathrm{H},\left(\mathrm{CH}_{3}\right)_{2} \mathrm{~N}\right), 2.16-1.95(\mathrm{~m}, 3 \mathrm{H}), 1.28$ (s, 12H, Bpin); ${ }^{13} \mathrm{C}$ NMR (100 $\left.\mathrm{MHz}, \mathrm{CDCl}_{3}\right): \delta 147.5,143.4,128.8,128.6,127.5,126.7,126.5,124.4,83.4,62.6,52.3,46.9$, 45.5, 28.4, 25.0, 24.9; IR v 2974 (w), 1348 (m), 1143 (s), 847 (w), 698 (s); HRMS (ESI) calcd. for $\mathrm{C}_{26} \mathrm{H}_{37} \mathrm{BNO}_{2}{ }^{+}[\mathrm{M}+\mathrm{H}]^{+}$406.2912; found: 406.2900. Carbon attached to boron not observed due to quadrupolar relaxation and another carbon was not resolved at $100 \mathrm{MHz}$. See spectra

$N, N$-dimethyl-1-((1r,4r,5s)-1-phenyl-1-(4,4,5,5-tetramethyl-1,3,2-dioxaborolan-2yl)spiro[3.5]nonan-5-yl)methanamine (1af)

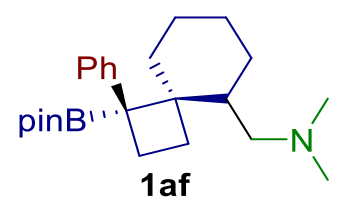

Following general procedure F, using 2-(1-(cyclohex-1-en-1-yl)cyclopropyl)-4,4,5,5tetramethyl-1,3,2-dioxaborolane (4i) (74.5 mg, $0.300 \mathrm{mmol}, 1.00$ equiv), $\mathrm{PhLi}$ (206 $\mu \mathrm{L}, 0.390$ mmol, 1.90 M in dibutyl ether, 1.30 equiv), and Eschenmoser's salt (5) (111 mg, $0.600 \mathrm{mmol}$, 
2.00 equiv). The crude material was purified by a quick flash chromatography using MeOH:DCM 1:15 as mobile phase to afford 1af $(88.0 \mathrm{mg}, 0.230 \mathrm{mmol}, 77 \%,>20: 1 \mathrm{dr})$ as a colorless liquid. TLC (MeOH:DCM, 1:10 v/v): $\mathrm{R}_{\mathrm{f}}=0.25, \mathrm{KMnO}_{4} ;{ }^{1} \mathrm{H}$ NMR $(400 \mathrm{MHz}$, $\left.\mathrm{CDCl}_{3}\right): \delta 7.51-7.43(\mathrm{~m}, 2 \mathrm{H}, \mathrm{ArH}), 7.24(\mathrm{t}, J=7.7 \mathrm{~Hz}, 2 \mathrm{H}, \mathrm{ArH}), 7.16-7.08(\mathrm{~m}, 1 \mathrm{H}, \mathrm{ArH})$, $2.52-2.41(\mathrm{~m}, 2 \mathrm{H}), 2.29-2.19(\mathrm{~m}, 7 \mathrm{H}), 2.18-2.02$ (m, 2H), $1.97-1.73(\mathrm{~m}, 2 \mathrm{H}), 1.57$ (dt, $J$ $=13.9,5.0 \mathrm{~Hz}, 1 \mathrm{H}), 1.49-1.27(\mathrm{~m}, 4 \mathrm{H}), 1.21(\mathrm{~s}, 6 \mathrm{H}), 1.19(\mathrm{~s}, 6 \mathrm{H}), 1.10-1.02(\mathrm{~m}, 1 \mathrm{H}), 0.99$ $-0.91(\mathrm{~m}, 1 \mathrm{H}), 0.68-0.56(\mathrm{~m}, 1 \mathrm{H}) ;{ }^{13} \mathrm{C} \mathrm{NMR}\left(100 \mathrm{MHz}, \mathrm{CDCl}_{3}\right): \delta 142.9,129.3,127.2,124.9$, 83.0, 58.6, 49.4, 45.9, 40.6, 31.8, 29.0, 25.1, 24.8, 24.3, 22.8, 21.3, 21.1; IR v 2974 (m), 2928 (s), 2854 (w), 1444 (m), 1377 (m), 1337 (s), 1299 (s), 1142 (s), 1127 (m), 964 (m), 851 (m); HRMS (ESI) calcd. for $\mathrm{C}_{24} \mathrm{H}_{39} \mathrm{BNO}_{2}{ }^{+}[\mathrm{M}+\mathrm{H}]^{+}$384.3068; found 384.3602. Carbon attached to boron not observed due to quadrupolar relaxation. $\underline{\text { See spectra }}$

\section{3-Cyclopropylidene- $N, N$-dimethylbutan-1-amine (2b)}

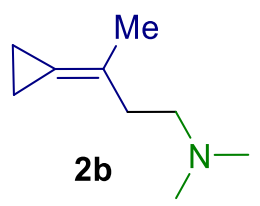

Following general procedure $\mathbf{F}$, using 4,4,5,5-tetramethyl-2-(1-(prop-1-en-2-yl)cyclopropyl)1,3,2-dioxaborolane (4h) (62.5 mg, 0.300 mmol, 1.00 equiv), PhLi (206 $\mu \mathrm{L}, 0.390 \mathrm{mmol}, 1.90$ $\mathrm{M}$ in dibutyl ether, 1.30 equiv), and Eschenmoser's salt (5) (111 mg, $0.600 \mathrm{mmol}, 2.00$ equiv). The solvent was removed at RT (product is volatile). The crude material was purified by a quick flash chromatography using MeOH:DCM 1:15 as mobile phase to afford $2 \mathbf{b}$ (26.5 mg, $0.190 \mathrm{mmol}, 63 \%)$ as a white amorphous solid. TLC (MeOH:DCM, 1:10 v/v): $\mathrm{R}_{\mathrm{f}}=0.22$, $\mathrm{KMnO}_{4} ;{ }^{1} \mathrm{H} \mathrm{NMR}\left(400 \mathrm{MHz}, \mathrm{CDCl}_{3}\right): \delta 3.26-3.13\left(\mathrm{~m}, 2 \mathrm{H}, \mathrm{CH}_{2}\left(\mathrm{CH}_{3}\right)_{2} \mathrm{~N}\right), 2.80(\mathrm{~s}, 6 \mathrm{H}$, $\left.\left(\mathrm{CH}_{3}\right)_{2} \mathrm{~N}\right), 2.71-2.65\left(\mathrm{~m}, 2 \mathrm{H}, \mathrm{CH}_{2} \mathrm{CH}_{2}\left(\mathrm{CH}_{3}\right)_{2} \mathrm{~N}\right), 1.84(\mathrm{p}, J=1.7 \mathrm{~Hz}, 3 \mathrm{H}), 1.12-1.06(\mathrm{~m}, 2 \mathrm{H})$, $1.02-0.94$ (m, 2H); $\left.{ }^{13} \mathrm{C} \mathrm{NMR} \mathrm{(100} \mathrm{MHz,} \mathrm{CDCl}_{3}\right): \delta$ 118.8, 118.6, 55.9, 42.8, 31.1, 21.1, 3.5, 1.6; IR v 2973 (w), 2659 (w), 2205 (w), 1477 (m), 1445 (w), 1188 (w), 958 (m), 922 (s); HRMS (ESI) calcd. for $\mathrm{C}_{9} \mathrm{H}_{18} \mathrm{~N}^{+}[\mathrm{M}+\mathrm{H}]^{+} 140.1433$; found 140.1430. Carbon attached to boron not observed due to quadrupolar relaxation. See spectra 
5. Stereoselective synthesis of $( \pm)$-grandisol

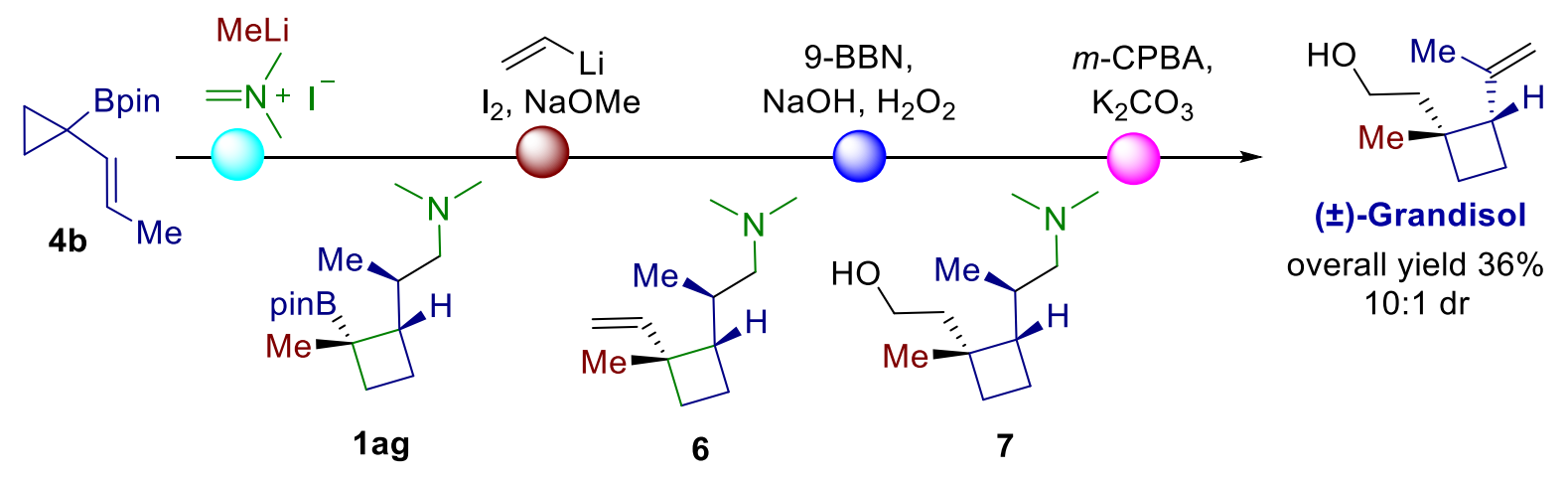

(r)- $N, N$-Dimethyl-2-((1s,2r)-2-methyl-2-(4,4,5,5-tetramethyl-1,3,2-dioxaborolan-2-

yl)cyclobutyl)propan-1-amine (1ag)

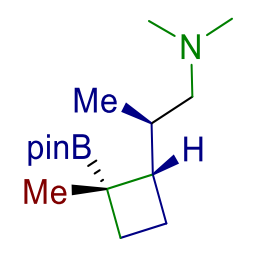

$1 \mathrm{ag}$

Following general procedure B, using (E)-4,4,5,5-tetramethyl-2-(1-(prop-1-en-1yl)cyclopropyl)-1,3,2-dioxaborolane (4b) (208 mg, 1.00 mmol, 1.00 equiv), MeLi (808 $\mu \mathrm{L}$, $1.30 \mathrm{mmol}, 1.61 \mathrm{M}$ in $\mathrm{Et}_{2} \mathrm{O}, 1.30$ equiv), and Eschenmoser's salt (5) (370 mg, $2.00 \mathrm{mmol}, 2.00$ equiv). The crude material was used in the next step without further purification. ${ }^{1} \mathrm{H}$ NMR (400 $\left.\mathrm{MHz}, \mathrm{CDCl}_{3}\right): \delta 2.18\left(\mathrm{~s}, 6 \mathrm{H},\left(\mathrm{CH}_{3}\right)_{2} \mathrm{~N}\right), 2.01-1.96(\mathrm{~m}, 1 \mathrm{H}), 1.96-1.86(\mathrm{~m}, 3 \mathrm{H}), 1.84-1.65$ (m, 2H), $1.56(\mathrm{td}, J=10.6,8.1 \mathrm{~Hz}, 1 \mathrm{H}), 1.41$ (td, $J=10.4,8.6 \mathrm{~Hz}, 1 \mathrm{H}), 1.27$ (s, 12H, Bpin), 1.15 (s, 3H, $\left.\mathrm{CH}_{2} \mathrm{CCH}_{3}\right), 0.88$ (d, $\left.\left.J=6.4 \mathrm{~Hz}, 3 \mathrm{H}, \mathrm{CHCH}_{3}\right) ;{ }^{13} \mathrm{C} \mathrm{NMR} \mathrm{(100} \mathrm{MHz,} \mathrm{CDCl}_{3}\right): \delta$ 82.8, 64.6, 52.7, 46.0, 36.7, 29.4, 26.7, 24.9, 24.8, 23.9, 16.4; IR v 2927 (s), 2857 (m), 2761 (w), 1462 (m), 1377 (s), 1303 (s), 1139 (s), 1032 (m), 847 (m); HRMS (ESI) calcd. for $\mathrm{C}_{16} \mathrm{H}_{33} \mathrm{BNO}_{2}{ }^{+}[\mathrm{M}+\mathrm{H}]^{+}$282.2601; found 282.2596. Carbon attached to boron not observed due to quadrupolar relaxation. See spectra 


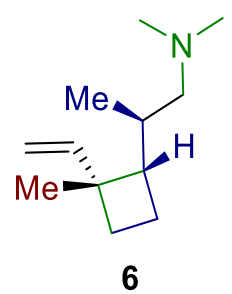

To neat tetravinyltin (227 mg, $1.00 \mathrm{mmol}, 1.00$ equiv) at RT was added $n \mathrm{BuLi}(1.25 \mathrm{~mL}, 2.00$ mmol, 2.00 equiv $1.60 \mathrm{M}$ in hexanes) dropwise over $10 \mathrm{~min}$. The resulting solution was stirred for $5 \mathrm{~min}$ at room temperature and then allowed to settle for $1 \mathrm{~h}$. The supernatant was removed carefully and the white solid was washed with dry hexane $(2 \times 2 \mathrm{~mL})$, every time adding and removing the solvent by syringe under $\mathrm{N}_{2}$. Attention being paid not to remove any of the white precipitate. The solid vinyl lithium was then dissolved in THF $(2 \mathrm{~mL})$. To a solution of (r)$N, N$-dimethyl-2-((1s,2r)-2-methyl-2-(4,4,5,5-tetramethyl-1,3,2-dioxaborolan-2-

yl)cyclobutyl)propan-1-amine (1ag) (from the previous step) in anhydrous THF (10 $\mathrm{mL}$ ) at $-78{ }^{\circ} \mathrm{C}$ was added dropwise the vinyl lithium solution. The reaction mixture was stirred at $-78{ }^{\circ} \mathrm{C}$ for $1 \mathrm{~h}$ and then warmed to RT and stirred for another $1 \mathrm{~h}$. The reaction mixture was then cooled down to $-78^{\circ} \mathrm{C}$ and a solution of iodine (508 mg, $2.00 \mathrm{mmol}, 2.00$ equiv) in $\mathrm{MeOH}$ $(10 \mathrm{~mL})$ was added dropwise to the reaction mixture. 30 min later, a solution of $\mathrm{MeONa}(12$ $\mathrm{ml}, 6.00 \mathrm{mmol}, 6.00$ equiv, $0.5 \mathrm{M}$ in $\mathrm{MeOH}$ ) was added. The reaction mixture was stirred at $-78{ }^{\circ} \mathrm{C}$ for $30 \mathrm{~min}$, then allowed to warm to RT and stirred for an additional $1 \mathrm{~h}$, then diluted with DCM (40 mL) and washed with a $20 \%$ aq. $\mathrm{Na}_{2} \mathrm{~S}_{2} \mathrm{O}_{3}$ solution $(20 \mathrm{~mL})$ and water $(20 \mathrm{~mL})$. The phases were separated, and the aqueous layer was extracted with $\mathrm{Et}_{2} \mathrm{O}(2 \times 20 \mathrm{~mL})$; the combined organic layers were washed with brine $(20 \mathrm{~mL})$, dried over $\mathrm{MgSO}_{4}$, filtered and concentrated in vacuo at RT (compound is highly volatile). The crude material was used for the next step without further purification. ${ }^{1} \mathrm{H}$ NMR (400 $\left.\mathrm{MHz}, \mathrm{CDCl}_{3}\right): \delta 6.16(\mathrm{dd}, J=17.3$, $\left.10.8 \mathrm{~Hz}, 1 \mathrm{H}, \mathrm{CH}=\mathrm{CH}_{2}\right), 5.21-4.96\left(\mathrm{~m}, 2 \mathrm{H}, \mathrm{CH}=\mathrm{CH}_{2}\right), 2.41\left(\mathrm{~s}, 6 \mathrm{H},\left(\mathrm{CH}_{3}\right)_{2} \mathrm{~N}\right), 2.27-2.15(\mathrm{~m}$, 2H), $2.07-1.95(\mathrm{~m}, 1 \mathrm{H}), 1.94-1.78(\mathrm{~m}, 2 \mathrm{H}), 1.76-1.68(\mathrm{~m}, 2 \mathrm{H}), 1.61$ (p, J=9.6 Hz, 1H), 1.21 (s, 3H), 0.93 (d, J=6.0 Hz, 3H); IR v 2924 (s), 2855 (m), 1673 (w), 1460 (m), 1366 (s), 1235 (w), 1142 (s), 1053 (w); HRMS (ESI) calcd. for $\mathrm{C}_{12} \mathrm{H}_{24} \mathrm{~N}^{+}[\mathrm{M}+\mathrm{H}]^{+}$182.1903; found 182.1907. See spectra 


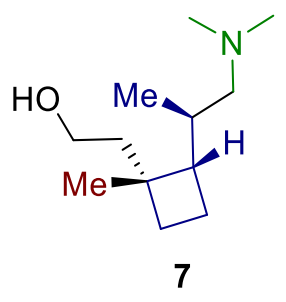

A round-bottom flask was charged with crude 6 under $\mathrm{N}_{2}$ atmosphere. Anhydrous tetrahydrofuran $(10 \mathrm{~mL})$ was added and the resulting solution was cooled to $0{ }^{\circ} \mathrm{C}$. A solution of 9-borabicyclo[3.3.1]nonane (10.0 mL, $1.00 \mathrm{mmol}, 5.00$ equiv, $0.5 \mathrm{M}$ in THF) was added drop-wise at $0{ }^{\circ} \mathrm{C}$. The reaction mixture was then allowed to warm to room temperature and stirred for $16 \mathrm{~h}$. At that time, the reaction solution was cooled to $0{ }^{\circ} \mathrm{C}$, and $2 \mathrm{M}$ aqueous $\mathrm{NaOH}$ (5.00 mL, $10.0 \mathrm{mmol}, 10.0$ equiv) was added slowly, followed by $30 \%$ (v/v) aqueous hydrogen peroxide (4.12 mL, $40.0 \mathrm{mmol}, 40.0$ equiv). The reaction mixture was then allowed to warm to RT while stirring for $4 \mathrm{~h}$. The mixture was then diluted with water and extracted with ethyl acetate $(3 \times 15 \mathrm{~mL})$. The combined organic layers were washed with water $(20 \mathrm{~mL}), 10 \%$ aq. $\mathrm{Na}_{2} \mathrm{~S}_{2} \mathrm{O}_{3}(20 \mathrm{~mL})$, and brine $(20 \mathrm{~mL})$. The organic layer was then dried over $\mathrm{MgSO}_{4}$, filtered, and concentrated under vacuo. The crude material was used in the next step without further purification. ${ }^{1} \mathrm{H}$ NMR (400 MHz, $\left.\mathrm{CDCl}_{3}\right): \delta 3.79-3.64\left(\mathrm{~m}, 2 \mathrm{H}, \mathrm{CH}_{2} \mathrm{OH}\right), 2.16(\mathrm{~s}, 6 \mathrm{H}$, $\left.\left(\mathrm{CH}_{3}\right)_{2} \mathrm{~N}\right), 1.96-1.86(\mathrm{~m}, 2 \mathrm{H}), 1.76-1.48(\mathrm{~m}, 8 \mathrm{H}), 1.06\left(\mathrm{~s}, 3 \mathrm{H}, \mathrm{CH}_{2} \mathrm{CCH}_{3}\right), 0.85(\mathrm{~d}, J=5.9$ $\left.\mathrm{Hz}, 3 \mathrm{H}, \mathrm{CHCH}_{3}\right) ;{ }^{13} \mathrm{C} \mathrm{NMR}\left(100 \mathrm{MHz}, \mathrm{CDCl}_{3}\right): \delta 64.2,60.1,52.1,46.0,40.1,36.4,32.4,29.7$, 29.7, 28.6, 21.9, 17.7; IR v 3323 (m), 2949 (m), 2863 (s), 2814 (m), 1458 (m), 1378 (m), 1150 (m), 1050 (s), 1035 (s), 846 (m); HRMS (ESI) calcd. for $\mathrm{C}_{12} \mathrm{H}_{26} \mathrm{NO}^{+}[\mathrm{M}+\mathrm{H}]^{+}$200.2009; found 200.2007. Carbon attached to boron not observed due to quadrupolar relaxation. See spectra

\section{( \pm )-Grandisol}

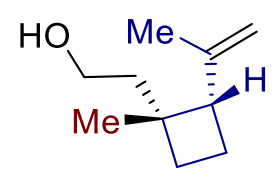

(士)-Grandisol

Following a modified reported procedure, ${ }^{3}$ a Schlenk tube was charged with crude $7, \mathrm{~K}_{2} \mathrm{CO}_{3}$ (207 mg, $1.50 \mathrm{mmol}, 1.50$ equiv), DMF (10 mL) and a magnetic stir bar under $\mathrm{N}_{2}$ atmosphere. $m$-CPBA (207 mg, $1.20 \mathrm{mmol}, 1.20$ equiv, 77\%) was added, and the reaction mixture was 
stirred vigorously for $20 \mathrm{~min}$ at $\mathrm{RT}$, then heated to $120^{\circ} \mathrm{C}$ for $12 \mathrm{~h}$. The reaction was allowed to cool to RT and then was diluted with water $(30 \mathrm{~mL})$. The solution was extracted with $\mathrm{Et}_{2} \mathrm{O}$ $(2 \times 25 \mathrm{~mL})$. The combined organic layers were then washed with brine $(25 \mathrm{~mL})$, dried over $\mathrm{MgSO}_{4}$, and concentrated under vacuum. The crude material was purified by flash column chromatography on silica gel using $\mathrm{Et}_{2} \mathrm{O}$ :pentane 1:20 as mobile phase to afford ( \pm )-grandisol as a colorless oil $(55.0 \mathrm{mg}, 0.357 \mathrm{mmol}, 36 \%$ yield over 4 steps). TLC (Et $2 \mathrm{O}$ :pentane, 1:20 $\mathrm{v} / \mathrm{v}): \mathrm{R}_{\mathrm{f}}=0.24, \mathrm{KMnO}_{4} ;{ }^{1} \mathrm{H} \mathrm{NMR}\left(400 \mathrm{MHz}, \mathrm{CDCl}_{3}\right): \delta 4.83(\mathrm{q}, J=1.4 \mathrm{~Hz}, 1 \mathrm{H}), 4.64$ (br s, 1H), $3.75-3.58(\mathrm{~m}, 2 \mathrm{H}), 2.54(\mathrm{t}, J=9.1 \mathrm{~Hz}, 1 \mathrm{H}), 2.04-1.90(\mathrm{~m}, 1 \mathrm{H}), 1.85-1.71(\mathrm{~m}, 2 \mathrm{H})$, $1.70-1.56(\mathrm{~m}, 6 \mathrm{H}), 1.48-1.38(\mathrm{~m}, 1 \mathrm{H}), 1.17(\mathrm{~s}, 3 \mathrm{H}) ;{ }^{13} \mathrm{C} \mathrm{NMR}\left(100 \mathrm{MHz}, \mathrm{CDCl}_{3}\right): \delta 145.2$, 109.7, 59.9, 52.4, 41.3, 36.9, 29.3, 28.3, 23.2, 19.1; IR v 3322 (m), 3079 (s), 2947 (s), 2866 (m), 1645 (m), 1453 (m), 1440 (m), 1238 (w), 1052 (s), 1000 (m), 883 (s); HRMS (APCI) calcd. for $\mathrm{C}_{10} \mathrm{H}_{19} \mathrm{O}^{+}[\mathrm{M}+\mathrm{H}]^{+}$155.1430; found 155.1430. Carbon attached to boron not observed due to quadrupolar relaxation. See spectra 


\section{DFT Calculations}

Calculations were performed using the Gaussian094 suite of programmes. Geometries were optimized with the DFT method using M06-2X functional as implemented in Gaussian, using 6-311G(d,p) as basis set. All calculations were carried out using the PCM model of solvation with tetrahydrofuran as the solvent. ${ }^{5}$ All geometry optimizations were full, with no restrictions. All stationary points located in the potential energy hypersurface were characterized as minima (no imaginary frequencies) or as transition states (one and only one imaginary frequency) by vibrational analysis. The analysis also provided zero-point vibrational energy corrections and thermal corrections to various thermodynamic properties. The transition state was further confirmed by IRC calculations. Full Cartesian coordinates for the optimised geometries are reported below.

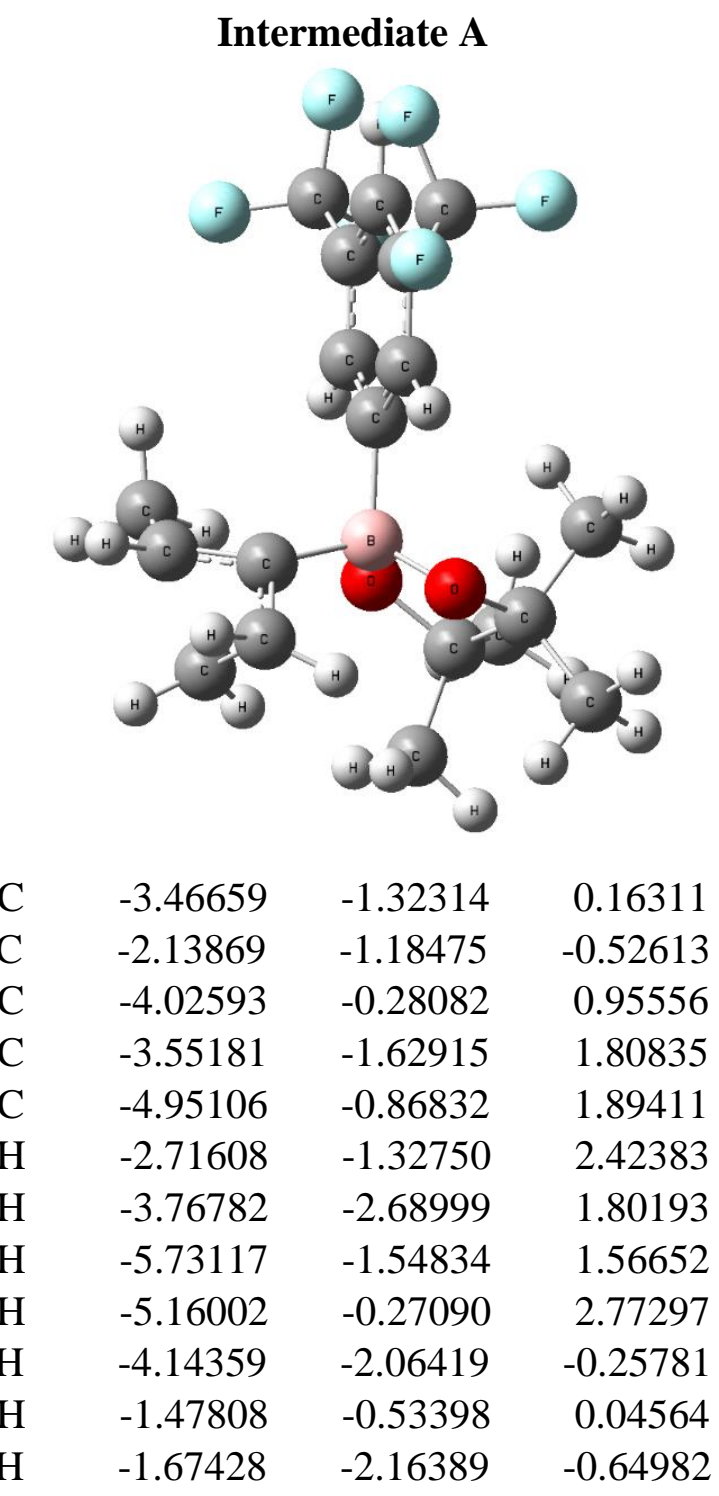




\begin{tabular}{|c|c|c|c|}
\hline $\mathrm{C}$ & -2.99281 & 2.54798 & 3.11775 \\
\hline $\mathrm{C}$ & -1.72259 & 1.64788 & 2.96724 \\
\hline $\mathrm{O}$ & -3.97820 & 1.79673 & 2.41399 \\
\hline $\mathrm{O}$ & -1.88562 & 1.07037 & 1.66919 \\
\hline B & -3.29415 & 1.18209 & 1.28023 \\
\hline $\mathrm{H}$ & -2.28843 & -0.75608 & -1.52043 \\
\hline $\mathrm{C}$ & -0.40191 & 2.40533 & 3.0080 \\
\hline $\mathrm{H}$ & -0.29432 & 2.95061 & 3.95005 \\
\hline $\mathrm{H}$ & 0.42670 & 1.69782 & 2.92728 \\
\hline $\mathrm{H}$ & -0.32994 & 3.11140 & 2.18092 \\
\hline $\mathrm{C}$ & -1.69828 & 0.52875 & 4.01114 \\
\hline $\mathrm{H}$ & -0.95179 & -0.21263 & 3.71520 \\
\hline $\mathrm{H}$ & -1.43225 & 0.90819 & 5.00068 \\
\hline $\mathrm{H}$ & -2.67305 & 0.04069 & 4.08631 \\
\hline $\mathrm{C}$ & -2.81292 & 3.91370 & 2.44516 \\
\hline $\mathrm{H}$ & -3.79204 & 4.39107 & 2.36255 \\
\hline $\mathrm{H}$ & -2.15718 & 4.56109 & 3.03246 \\
\hline $\mathrm{H}$ & -2.39645 & 3.81389 & 1.44131 \\
\hline $\mathrm{C}$ & -3.45766 & 2.75292 & 4.55272 \\
\hline $\mathrm{H}$ & -2.67059 & 3.21764 & 5.15322 \\
\hline $\mathrm{H}$ & -4.32718 & 3.41399 & 4.56046 \\
\hline $\mathrm{H}$ & -3.74357 & 1.80856 & 5.01633 \\
\hline $\mathrm{C}$ & -3.49328 & 1.98392 & -0.13217 \\
\hline $\mathrm{C}$ & -4.73189 & 2.52961 & -0.4845 \\
\hline $\mathrm{C}$ & -2.44706 & 2.13601 & -1.04164 \\
\hline $\mathrm{C}$ & -4.91299 & 3.19007 & -1.69519 \\
\hline $\mathrm{H}$ & -5.56673 & 2.44473 & 0.20502 \\
\hline $\mathrm{C}$ & -2.63413 & 2.78629 & -2.2596 \\
\hline $\mathrm{H}$ & -1.46434 & 1.75028 & -0.7857 \\
\hline $\mathrm{C}$ & -3.86711 & 3.32080 & -2.6028 \\
\hline $\mathrm{H}$ & -4.01243 & 3.82428 & -3.5497 \\
\hline $\mathrm{C}$ & -1.47830 & 2.86059 & -3.2090 \\
\hline $\mathrm{C}$ & -6.23674 & 3.82109 & -2.00263 \\
\hline $\mathrm{F}$ & -1.14299 & 1.64247 & -3.67376 \\
\hline $\mathrm{F}$ & -1.73077 & 3.62754 & -4.27767 \\
\hline $\mathrm{F}$ & -0.37687 & 3.35302 & -2.61979 \\
\hline $\mathrm{F}$ & -6.33254 & 5.05879 & -1.48511 \\
\hline $\mathrm{F}$ & -6.44954 & 3.94116 & -3.3217 \\
\hline $\mathrm{F}$ & -7.26197 & 3.11814 & -1.4989 \\
\hline
\end{tabular}


TS mig1 $_{\text {(Transition state for migration from Int. A) }}$

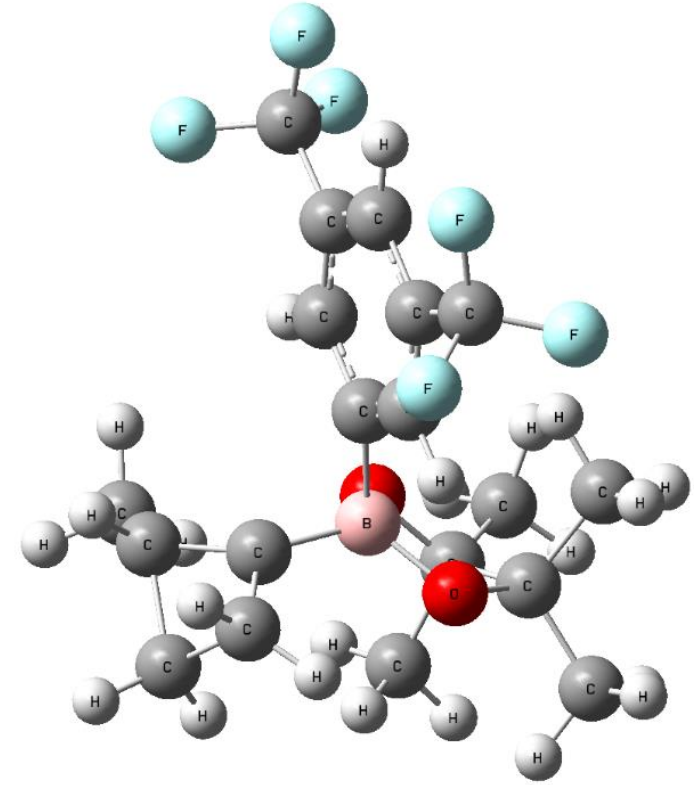

$\begin{array}{llll}\text { C } & -3.37021 & -1.38259 & 0.29548\end{array}$

$\begin{array}{llll}\text { C } & -1.87741 & -1.57159 & 0.19540\end{array}$

$\begin{array}{llll}\text { C } & -3.94130 & -0.17393 & 0.87548\end{array}$

$\begin{array}{llll}\text { C } & -4.18910 & -1.83759 & 1.60061\end{array}$

$\begin{array}{llll}\text { C } & -5.23105 & -0.67436 & 1.34215\end{array}$

$\begin{array}{llll}\mathrm{H} & -3.63471 & -1.76600 & 2.53027\end{array}$

$\mathrm{H} \quad-4.62807 \quad-2.82018 \quad 1.45107$

$\begin{array}{llll}\mathrm{H} & -5.94407 & -1.02787 & 0.59987\end{array}$

$\mathrm{H} \quad-5.68055 \quad-0.15325 \quad 2.18112$

$\mathrm{H} \quad-3.93436 \quad-1.80685 \quad-0.53710$

$\mathrm{H} \quad-1.37650 \quad-1.10198 \quad 1.04117$

$\begin{array}{llll}\mathrm{H} & -1.63396 & -2.63507 & 0.16461\end{array}$

$\begin{array}{llll}\text { C } & -2.88104 & 2.56357 & 3.15248\end{array}$

$\begin{array}{llll}\text { C } & -1.57673 & 1.77324 & 2.81422\end{array}$

$\begin{array}{llll}\text { O } & -3.88745 & 1.75988 & 2.53152\end{array}$

$\begin{array}{llll}\text { O } & -1.83811 & 1.29912 & 1.49066\end{array}$

B $\quad-3.27066 \quad 1.17011 \quad 1.36810$

$\begin{array}{llll}\mathrm{H} & -1.49985 & -1.11370 & -0.72161\end{array}$

$\begin{array}{llll}\text { C } & -0.31246 & 2.61798 & 2.79872\end{array}$

$\mathrm{H} \quad-0.14821 \quad 3.08642 \quad 3.77311$

$\begin{array}{llll}\mathrm{H} & 0.54841 & 1.98474 & 2.57307\end{array}$

$\begin{array}{llll}\mathrm{H} & -0.37450 & 3.39587 & 2.03784\end{array}$

$\begin{array}{llll}\text { C } & -1.39127 & 0.56159 & 3.73029\end{array}$

$\mathrm{H} \quad-0.60157 \quad-0.07161 \quad 3.31982$

$\begin{array}{llll}\mathrm{H} & -1.10641 & 0.85637 & 4.74306\end{array}$

$\begin{array}{llll}\mathrm{H} & -2.31386 & -0.02232 & 3.78522\end{array}$

$\begin{array}{llll}\text { C } & -2.89065 & 3.95054 & 2.50492\end{array}$

$\begin{array}{llll}\mathrm{H} & -3.90218 & 4.35779 & 2.56913\end{array}$

$\mathrm{H} \quad-2.20615 \quad 4.63595 \quad 3.01040$

$\mathrm{H} \quad-2.61061 \quad 3.88896 \quad 1.45066$ 


$\begin{array}{rrrr}\text { C } & -3.18695 & 2.67620 & 4.63777 \\ \text { H } & -2.37215 & 3.18047 & 5.16469 \\ \text { H } & -4.09880 & 3.26061 & 4.77889 \\ \text { H } & -3.33867 & 1.69184 & 5.08070 \\ \text { C } & -3.80619 & 1.82059 & -0.10271 \\ \text { C } & -5.06240 & 2.41405 & -0.21741 \\ \text { C } & -2.93923 & 1.88701 & -1.19319 \\ \text { C } & -5.42748 & 3.08286 & -1.38165 \\ \text { H } & -5.75330 & 2.36904 & 0.62014 \\ \text { C } & -3.31284 & 2.55192 & -2.35685 \\ \text { H } & -1.95169 & 1.44108 & -1.11569 \\ \text { C } & -4.55865 & 3.15790 & -2.46319 \\ \text { H } & -4.84626 & 3.67536 & -3.36877 \\ \text { C } & -2.34235 & 2.59869 & -3.49893 \\ \text { C } & -6.77298 & 3.74197 & -1.43863 \\ \text { F } & -2.03693 & 1.36612 & -3.93827 \\ \text { F } & -2.81484 & 3.28409 & -4.54729 \\ \text { F } & -1.18139 & 3.17034 & -3.14300 \\ \text { F } & -6.85176 & 4.78503 & -0.59628 \\ \text { F } & -7.06928 & 4.20187 & -2.66046 \\ \text { F } & -7.75357 & 2.89570 & -1.08450 \\ & \text { G }=-1511.780199 \text { Hartree }\end{array}$

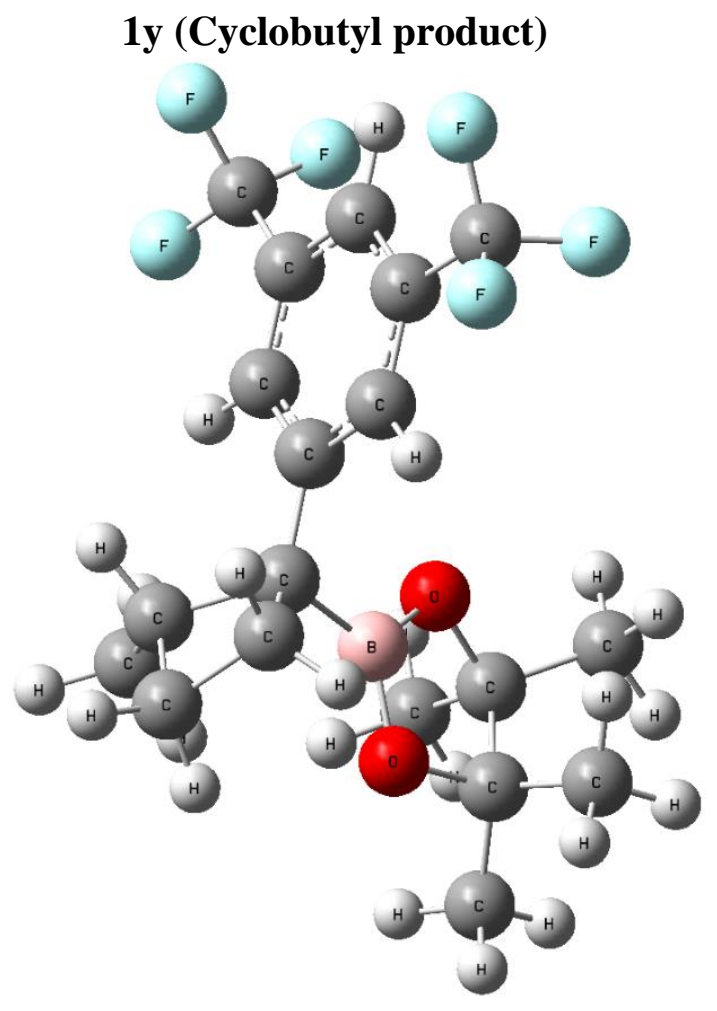

$\begin{array}{lrrr}\mathrm{C} & -3.79246 & -1.25383 & 0.68274 \\ \mathrm{C} & -2.68793 & -1.88123 & 1.51563 \\ \mathrm{C} & -3.77478 & 0.29000 & 0.37940\end{array}$




\begin{tabular}{|c|c|c|c|}
\hline $\mathrm{C}$ & -5.17464 & -1.06026 & 1.34256 \\
\hline $\mathrm{C}$ & -5.31755 & 0.28145 & 0.59564 \\
\hline $\mathrm{H}$ & -5.06646 & -0.90133 & 2.41743 \\
\hline $\mathrm{H}$ & -5.93020 & -1.82396 & 1.15990 \\
\hline $\mathrm{H}$ & -5.83785 & 0.15564 & -0.35694 \\
\hline $\mathrm{H}$ & -5.75441 & 1.11790 & 1.14175 \\
\hline $\mathrm{H}$ & -3.90254 & -1.78197 & -0.2702 \\
\hline $\mathrm{H}$ & -2.63647 & -1.40392 & 2.49872 \\
\hline $\mathrm{H}$ & -2.88529 & -2.94428 & 1.67402 \\
\hline $\mathrm{C}$ & -2.64549 & 2.16442 & 3.47410 \\
\hline $\mathrm{C}$ & -1.31968 & 2.07645 & 2.6485 \\
\hline $\mathrm{O}$ & -3.49255 & 1.19678 & $2.7967^{\prime}$ \\
\hline $\mathrm{O}$ & -1.80177 & 1.67018 & 1.3405 \\
\hline B & -3.01427 & 1.06743 & 1.52046 \\
\hline $\mathrm{H}$ & -1.70781 & -1.78780 & 1.03932 \\
\hline $\mathrm{C}$ & -0.56840 & 3.38843 & $2.5085^{\circ}$ \\
\hline $\mathrm{H}$ & -0.27296 & 3.76334 & 3.49172 \\
\hline $\mathrm{H}$ & 0.33516 & 3.22619 & 1.91838 \\
\hline $\mathrm{H}$ & -1.17514 & 4.14396 & 2.0107 \\
\hline $\mathrm{C}$ & -0.38432 & 0.97584 & 3.1372 \\
\hline $\mathrm{H}$ & 0.40746 & 0.83711 & 2.3992 \\
\hline $\mathrm{H}$ & 0.07099 & 1.24150 & 4.09317 \\
\hline $\mathrm{H}$ & -0.91608 & 0.02961 & 3.25309 \\
\hline $\mathrm{C}$ & -3.33758 & 3.51693 & 3.3407 \\
\hline $\mathrm{H}$ & -4.33764 & 3.44244 & 3.77102 \\
\hline $\mathrm{H}$ & -2.78716 & 4.29813 & 3.8685 \\
\hline $\mathrm{H}$ & -3.43379 & 3.80357 & 2.2909 \\
\hline $\mathrm{C}$ & -2.51281 & 1.77060 & 4.9335 \\
\hline $\mathrm{H}$ & -1.80791 & 2.43386 & 5.44130 \\
\hline $\mathrm{H}$ & -3.48354 & 1.86222 & 5.4239 \\
\hline $\mathrm{H}$ & -2.16715 & 0.74261 & 5.0385 \\
\hline $\mathrm{C}$ & -3.27247 & 0.71798 & -0.96942 \\
\hline $\mathrm{C}$ & -3.89891 & 1.75229 & -1.6620 \\
\hline $\mathrm{C}$ & -2.15587 & 0.11210 & -1.5509 \\
\hline $\mathrm{C}$ & -3.42417 & 2.16411 & -2.9032 \\
\hline $\mathrm{H}$ & -4.77211 & 2.23448 & -1.2360 \\
\hline $\mathrm{C}$ & -1.69267 & 0.53019 & -2.7899 \\
\hline $\mathrm{H}$ & -1.64975 & -0.69576 & -1.0355 \\
\hline $\mathrm{C}$ & -2.31889 & 1.56098 & -3.4826 \\
\hline $\mathrm{H}$ & -1.96109 & 1.87630 & -4.4553 \\
\hline $\mathrm{C}$ & -0.48508 & -0.10733 & -3.4112 \\
\hline $\mathrm{C}$ & -4.08564 & 3.31320 & -3.6050 \\
\hline $\mathrm{F}$ & -0.72290 & -0.49624 & -4.6736 \\
\hline $\mathrm{F}$ & 0.55038 & 0.74636 & -3.4668 \\
\hline $\mathrm{F}$ & -0.06856 & -1.18493 & -2.7363 \\
\hline $\mathrm{F}$ & -3.53194 & 4.48828 & -3.2622 \\
\hline
\end{tabular}




$$
\begin{array}{llrr}
F & -3.98416 & 3.21524 & -4.93758 \\
F & -5.38916 & 3.40208 & -3.30820 \\
\multicolumn{4}{c}{\text { G }=-1511.869589 \text { Hartree }}
\end{array}
$$

TS $_{\gamma} 1$ (Transition state for $\gamma$-addition from Int. A)

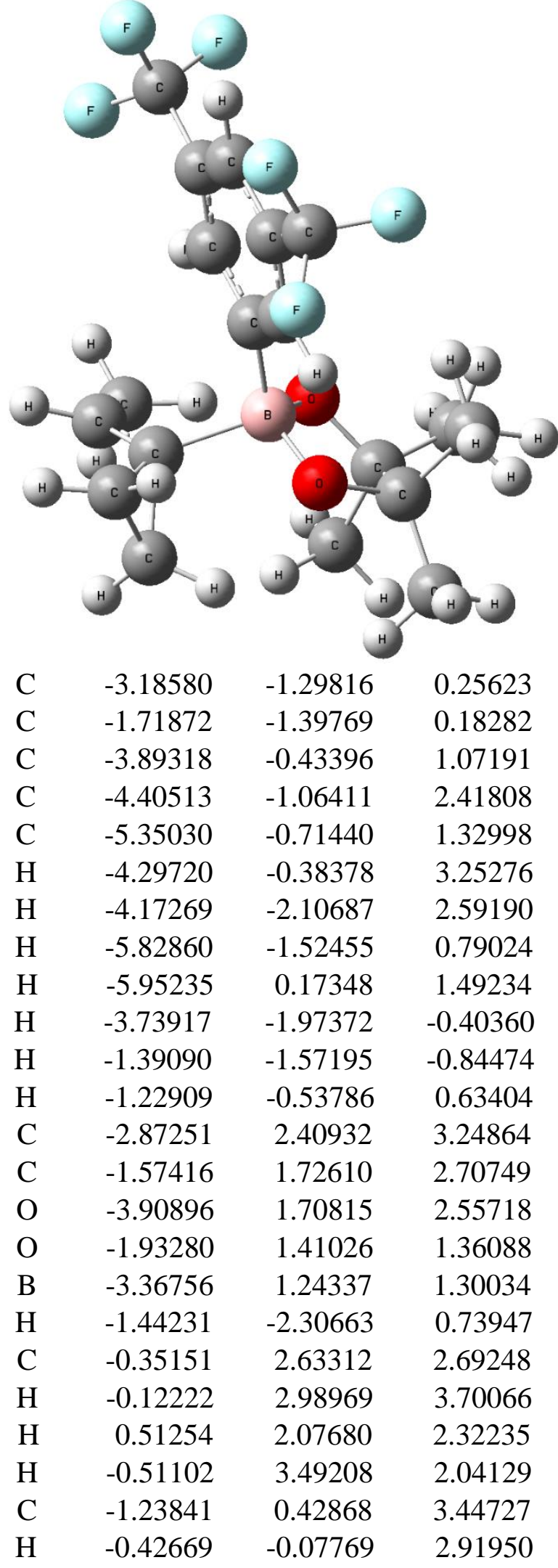




\begin{tabular}{lrrr} 
H & -0.90995 & 0.62271 & 4.47091 \\
H & -2.09810 & -0.24335 & 3.47757 \\
C & -2.95333 & 3.88499 & 2.85127 \\
H & -3.95807 & 4.25025 & 3.07516 \\
H & -2.23221 & 4.49373 & 3.40156 \\
H & -2.77430 & 4.00600 & 1.78026 \\
C & -3.08506 & 2.26012 & 4.74740 \\
H & -2.25524 & 2.70529 & 5.30358 \\
H & -4.00506 & 2.77199 & 5.03764 \\
H & -3.17164 & 1.21006 & 5.02907 \\
C & -4.02236 & 1.96246 & -0.00445 \\
C & -5.07868 & 2.86406 & 0.12226 \\
C & -3.54210 & 1.71297 & -1.29097 \\
C & -5.63614 & 3.48280 & -0.99521 \\
H & -5.47196 & 3.07772 & 1.11132 \\
C & -4.09214 & 2.33840 & -2.40397 \\
H & -2.72498 & 1.00900 & -1.43477 \\
C & -5.14957 & 3.23145 & -2.26995 \\
H & -5.58906 & 3.70645 & -3.13740 \\
C & -3.50542 & 2.07361 & -3.75701 \\
C & -6.74519 & 4.47191 & -0.80316 \\
F & -3.15429 & 0.78592 & -3.90867 \\
F & -4.35468 & 2.37149 & -4.75022 \\
F & -2.39254 & 2.79637 & -3.97078 \\
F & -6.27919 & 5.67978 & -0.43928 \\
F & -7.46451 & 4.65855 & -1.91927 \\
F & -7.59918 & 4.09055 & 0.15895 \\
& G =-1511.772758 Hartree \\
\hline & & &
\end{tabular}




\section{2c (Products from $\gamma$-addition)}

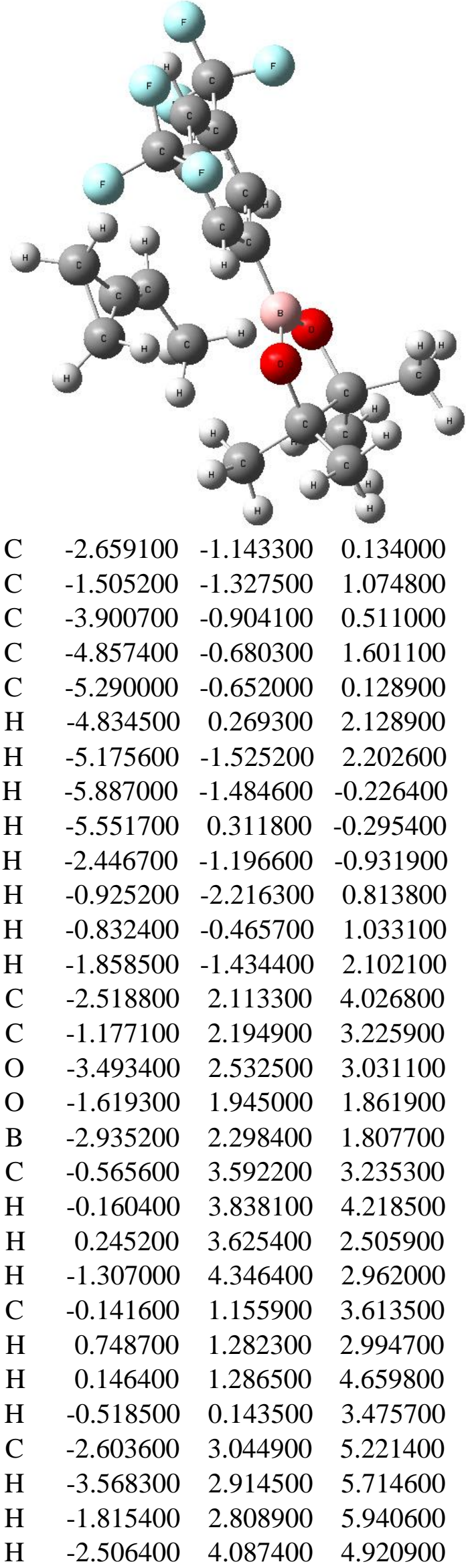




$\begin{array}{cccc}\mathrm{C} & -2.884600 & 0.688000 & 4.424100 \\ \mathrm{H} & -2.225900 & 0.316500 & 5.211300 \\ \mathrm{H} & -3.911800 & 0.677500 & 4.793200 \\ \mathrm{H} & -2.821800 & 0.018900 & 3.563000 \\ \mathrm{C} & -3.735400 & 2.451400 & 0.478500 \\ \mathrm{C} & -5.051700 & 2.914200 & 0.487900 \\ \mathrm{C} & -3.157300 & 2.108400 & -0.744000 \\ \mathrm{C} & -5.767700 & 3.030400 & -0.699400 \\ \mathrm{H} & -5.520300 & 3.181400 & 1.429800 \\ \mathrm{C} & -3.883500 & 2.216200 & -1.923500 \\ \mathrm{H} & -2.136400 & 1.740700 & -0.767600 \\ \mathrm{C} & -5.193400 & 2.679300 & -1.914200 \\ \mathrm{H} & -5.755600 & 2.760200 & -2.835500 \\ \mathrm{C} & -3.225600 & 1.801400 & -3.205800 \\ \mathrm{C} & -7.202900 & 3.459700 & -0.639700 \\ \mathrm{~F} & -2.096200 & 2.490500 & -3.429200 \\ \mathrm{~F} & -2.879500 & 0.503600 & -3.181900 \\ \mathrm{~F} & -4.016500 & 1.979500 & -4.269400 \\ \mathrm{~F} & -7.987500 & 2.472700 & -0.173200 \\ \mathrm{~F} & -7.378400 & 4.505700 & 0.179400 \\ \mathrm{~F} & -7.683300 & 3.805200 & -1.840000 \\ & \mathrm{G}=-1511.833992 \text { Hartree }\end{array}$

\section{Intermediate B}

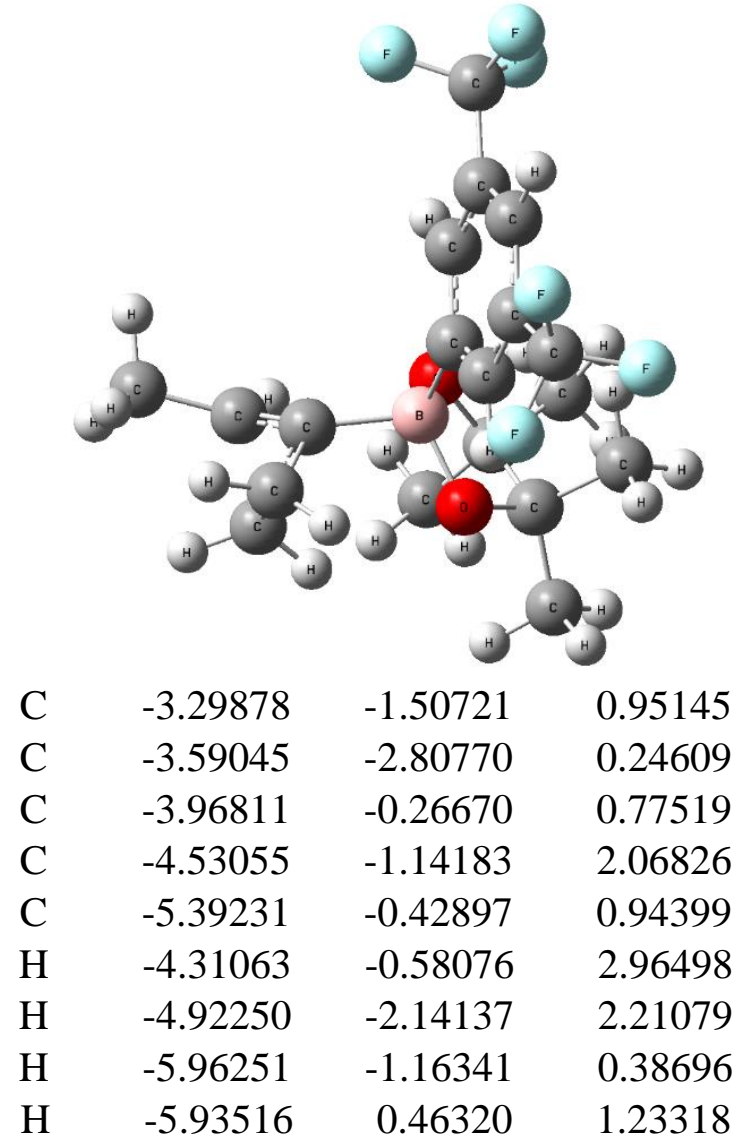




\begin{tabular}{|c|c|c|c|}
\hline $\mathrm{H}$ & -2.28206 & -1.38752 & 1.31901 \\
\hline $\mathrm{H}$ & -4.62318 & -2.87616 & -0.09610 \\
\hline $\mathrm{H}$ & -2.93647 & -2.87084 & -0.62554 \\
\hline $\mathrm{C}$ & -2.78154 & 2.39191 & 3.03892 \\
\hline $\mathrm{C}$ & -1.53959 & 1.48918 & 2.72159 \\
\hline $\mathrm{O}$ & -3.83339 & 1.73917 & 2.32920 \\
\hline $\mathrm{O}$ & -1.80570 & 1.02292 & 1.40045 \\
\hline B & -3.23071 & 1.17958 & 1.1203 \\
\hline $\mathrm{H}$ & -3.37030 & -3.65622 & 0.8946 \\
\hline $\mathrm{C}$ & -0.21043 & 2.23502 & 2.7281 \\
\hline $\mathrm{H}$ & -0.02466 & 2.69131 & 3.7048 \\
\hline $\mathrm{H}$ & 0.60069 & 1.53380 & 2.5191 \\
\hline $\mathrm{H}$ & -0.19393 & 3.01362 & 1.96576 \\
\hline $\mathrm{C}$ & -1.43604 & 0.28302 & 3.65885 \\
\hline $\mathrm{H}$ & -0.68451 & -0.40307 & 3.26103 \\
\hline $\mathrm{H}$ & -1.13518 & 0.57667 & 4.66739 \\
\hline $\mathrm{H}$ & -2.38471 & -0.25200 & 3.72757 \\
\hline $\mathrm{C}$ & -2.61576 & 3.81171 & 2.48767 \\
\hline $\mathrm{H}$ & -3.58279 & 4.31748 & 2.53887 \\
\hline $\mathrm{H}$ & -1.89075 & 4.38599 & 3.0692 \\
\hline $\mathrm{H}$ & -2.29358 & 3.79535 & 1.44474 \\
\hline $\mathrm{C}$ & -3.15019 & 2.45717 & 4.51394 \\
\hline $\mathrm{H}$ & -2.31770 & 2.84766 & 5.10590 \\
\hline $\mathrm{H}$ & -4.00568 & 3.12324 & 4.64611 \\
\hline $\mathrm{H}$ & -3.41997 & 1.47156 & 4.89432 \\
\hline $\mathrm{C}$ & -3.49277 & 2.05485 & -0.23390 \\
\hline $\mathrm{C}$ & -4.63269 & 2.85521 & -0.36202 \\
\hline $\mathrm{C}$ & -2.60104 & 2.03442 & -1.30600 \\
\hline $\mathrm{C}$ & -4.86884 & 3.59627 & -1.51411 \\
\hline $\mathrm{H}$ & -5.33595 & 2.91228 & 0.4633 \\
\hline $\mathrm{C}$ & -2.84216 & 2.77560 & -2.46195 \\
\hline $\mathrm{H}$ & -1.69380 & 1.44138 & -1.2312 \\
\hline $\mathrm{C}$ & -3.97752 & 3.56330 & -2.58142 \\
\hline $\mathrm{H}$ & -4.16594 & 4.13682 & -3.4797 \\
\hline $\mathrm{C}$ & -1.85977 & 2.68468 & -3.58958 \\
\hline $\mathrm{C}$ & -6.06301 & 4.49826 & -1.58510 \\
\hline $\mathrm{F}$ & -1.85512 & 1.46106 & -4.14792 \\
\hline $\mathrm{F}$ & -2.11795 & 3.55837 & -4.57227 \\
\hline $\mathrm{F}$ & -0.60257 & 2.91361 & -3.1777 \\
\hline $\mathrm{F}$ & -5.79704 & 5.71968 & -1.08902 \\
\hline $\mathrm{F}$ & -6.48661 & 4.68281 & -2.8448 \\
\hline $\mathrm{F}$ & -7.10151 & 4.02074 & -0.8835 \\
\hline \multicolumn{4}{|c|}{$\mathrm{G}=-1511.781741$ Hartree } \\
\hline
\end{tabular}




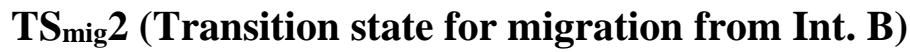

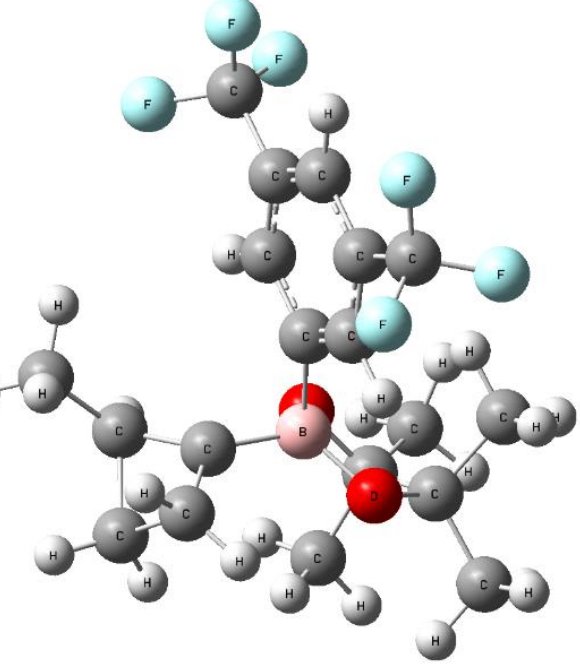

$\begin{array}{rrrr}\text { C } & -3.21179 & -1.28496 & 0.33195 \\ \mathrm{C} & -3.61754 & -1.94211 & -0.97506 \\ \mathrm{C} & -3.93757 & -0.15681 & 0.90294 \\ \mathrm{C} & -4.04809 & -1.84515 & 1.57719 \\ \mathrm{C} & -5.19064 & -0.75412 & 1.32886 \\ \mathrm{H} & -3.55510 & -1.78930 & 2.53985 \\ \mathrm{H} & -4.42216 & -2.84081 & 1.34953 \\ \mathrm{H} & -5.88015 & -1.15472 & 0.59105 \\ \mathrm{H} & -5.68030 & -0.28598 & 2.17707 \\ \mathrm{H} & -2.13310 & -1.20527 & 0.45816 \\ \mathrm{H} & -4.70133 & -2.00549 & -1.08281 \\ \mathrm{H} & -3.22473 & -1.35766 & -1.80998 \\ \mathrm{C} & -2.89054 & 2.57968 & 3.19258 \\ \mathrm{C} & -1.61051 & 1.73815 & 2.88255 \\ \mathrm{O} & -3.91533 & 1.80521 & 2.56189 \\ \mathrm{O} & -1.86373 & 1.27044 & 1.55449 \\ \mathrm{~B} & -3.29886 & 1.19520 & 1.41049 \\ \mathrm{H} & -3.20253 & -2.94931 & -1.03493 \\ \mathrm{C} & -0.31360 & 2.53188 & 2.89051 \\ \mathrm{H} & -0.15007 & 2.99713 & 3.86654 \\ \mathrm{H} & 0.52561 & 1.86372 & 2.68470 \\ \mathrm{H} & -0.32879 & 3.30890 & 2.12613 \\ \mathrm{C} & -1.49466 & 0.52487 & 3.80747 \\ \mathrm{H} & -0.73930 & -0.15287 & 3.40413 \\ \mathrm{H} & -1.20018 & 0.81150 & 4.81987 \\ \mathrm{H} & -2.44846 & -0.00615 & 3.86169 \\ \mathrm{C} & -2.83603 & 3.95944 & 2.53292 \\ \mathrm{H} & -3.83162 & 4.40693 & 2.57601 \\ \mathrm{H} & -2.13434 & 4.62143 & 3.04580 \\ \mathrm{H} & -2.53888 & 3.87879 & 1.48483 \\ \mathrm{C} & -3.21714 & 2.71839 & 4.67112 \\ \mathrm{H} & -2.39704 & 3.20536 & 5.20598 \\ & & & \end{array}$




$\begin{array}{lllr}\text { H } & -4.11375 & 3.33091 & 4.78959 \\ \text { H } & -3.40435 & 1.74422 & 5.12259 \\ \text { C } & -3.81255 & 1.83706 & -0.06583 \\ \text { C } & -5.02024 & 2.52885 & -0.15105 \\ \text { C } & -3.01688 & 1.75143 & -1.20819 \\ \text { C } & -5.40270 & 3.14502 & -1.33833 \\ \text { H } & -5.65500 & 2.59762 & 0.72812 \\ \text { C } & -3.41175 & 2.35725 & -2.39670 \\ \text { H } & -2.07472 & 1.21138 & -1.16454 \\ \text { C } & -4.60655 & 3.06271 & -2.47402 \\ \text { H } & -4.91407 & 3.53029 & -3.40015 \\ \text { C } & -2.51342 & 2.24824 & -3.59195 \\ \text { C } & -6.68797 & 3.91695 & -1.36323 \\ \text { F } & -2.11068 & 0.98189 & -3.79193 \\ \text { F } & -3.10712 & 2.65768 & -4.71978 \\ \text { F } & -1.39837 & 2.98138 & -3.44636 \\ \text { F } & -6.62168 & 5.01155 & -0.58811 \\ \text { F } & -7.01818 & 4.32720 & -2.59419 \\ \text { F } & -7.71468 & 3.18665 & -0.89941 \\ & \text { G }=-1511.778204 \text { Hartree }\end{array}$

Intermediate $\mathbf{C}$

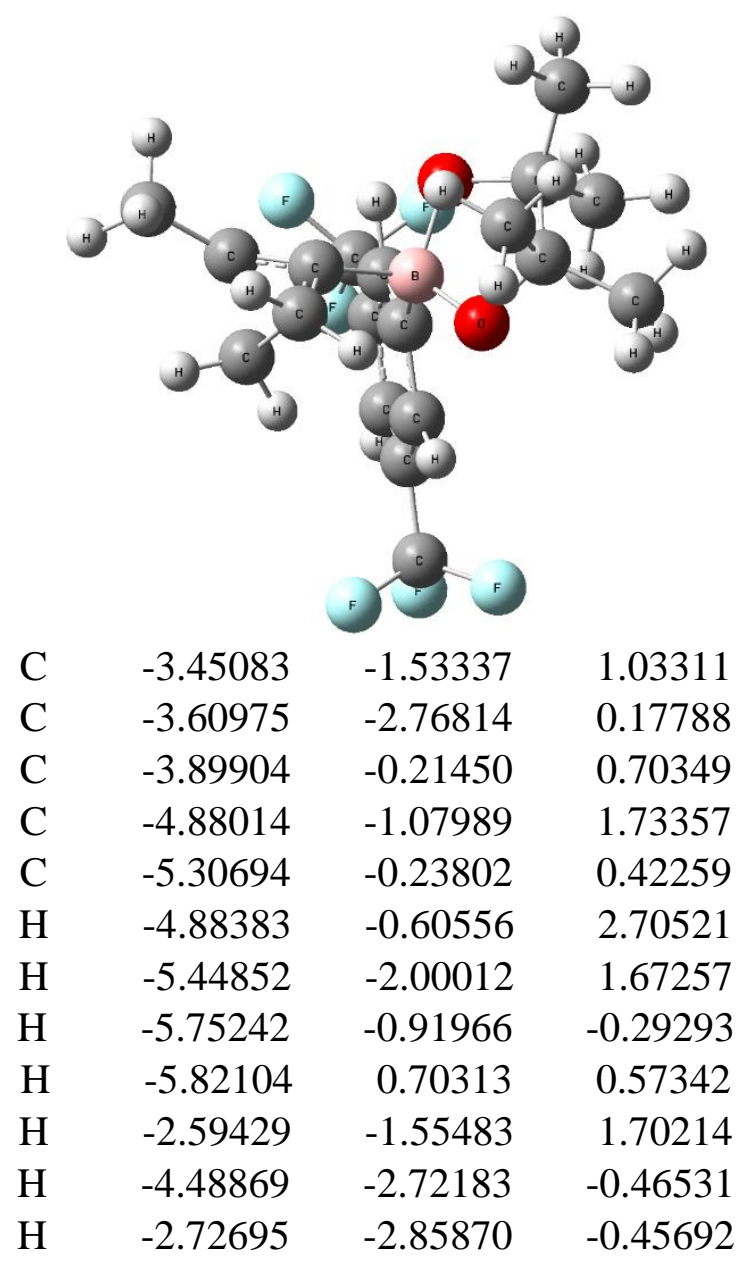




\begin{tabular}{|c|c|c|c|}
\hline $\mathrm{C}$ & -2.22986 & 2.84526 & -0.03849 \\
\hline $\mathrm{C}$ & -3.75113 & 3.14485 & 0.15642 \\
\hline $\mathrm{O}$ & -2.15517 & 1.44876 & 0.24230 \\
\hline $\mathrm{O}$ & -4.09363 & 2.30170 & 1.25696 \\
\hline B & -3.16308 & 1.17552 & 1.27059 \\
\hline $\mathrm{H}$ & -3.67242 & -3.66049 & 0.80156 \\
\hline $\mathrm{C}$ & -4.06559 & 4.58847 & 0.52121 \\
\hline $\mathrm{H}$ & -3.70127 & 5.27296 & -0.25025 \\
\hline $\mathrm{H}$ & -5.14686 & 4.71720 & 0.60983 \\
\hline $\mathrm{H}$ & -3.60925 & 4.85559 & 1.47413 \\
\hline $\mathrm{C}$ & -4.56807 & 2.73317 & -1.07200 \\
\hline $\mathrm{H}$ & -5.62986 & 2.75593 & -0.81463 \\
\hline $\mathrm{H}$ & -4.40452 & 3.41651 & -1.90873 \\
\hline $\mathrm{H}$ & -4.30663 & 1.72261 & -1.39450 \\
\hline $\mathrm{C}$ & -1.36768 & 3.60120 & 0.97748 \\
\hline $\mathrm{H}$ & -0.35847 & 3.18374 & 0.95625 \\
\hline $\mathrm{H}$ & -1.30916 & 4.66653 & 0.74128 \\
\hline $\mathrm{H}$ & -1.76566 & 3.48634 & 1.98807 \\
\hline $\mathrm{C}$ & -1.71019 & 3.09701 & -1.44614 \\
\hline $\mathrm{H}$ & -1.87299 & 4.13805 & -1.73917 \\
\hline $\mathrm{H}$ & -0.63704 & 2.89616 & -1.48021 \\
\hline $\mathrm{H}$ & -2.20311 & 2.44575 & -2.16813 \\
\hline $\mathrm{C}$ & -2.55009 & 0.93670 & 2.75710 \\
\hline $\mathrm{C}$ & -1.25662 & 0.43895 & 2.9352 \\
\hline $\mathrm{C}$ & -3.27637 & 1.24826 & 3.9092 \\
\hline $\mathrm{C}$ & -0.72387 & 0.24229 & 4.20639 \\
\hline $\mathrm{H}$ & -0.65293 & 0.21533 & 2.05966 \\
\hline $\mathrm{C}$ & -2.74325 & 1.04763 & 5.1798 \\
\hline $\mathrm{H}$ & -4.26978 & 1.67785 & 3.8080 \\
\hline $\mathrm{C}$ & -1.46101 & 0.54012 & 5.34508 \\
\hline $\mathrm{H}$ & -1.04504 & 0.38702 & 6.33204 \\
\hline $\mathrm{C}$ & -3.59013 & 1.37428 & 6.3718 \\
\hline $\mathrm{C}$ & 0.68067 & -0.26588 & 4.3264 \\
\hline $\mathrm{F}$ & -4.64905 & 0.54951 & 6.46872 \\
\hline $\mathrm{F}$ & -2.91715 & 1.27890 & $7.5260^{\circ}$ \\
\hline $\mathrm{F}$ & -4.08951 & 2.61863 & $6.3067 \S$ \\
\hline $\mathrm{F}$ & 1.58096 & 0.65780 & 3.95022 \\
\hline $\mathrm{F}$ & 0.99292 & -0.62618 & $5.5788^{\circ}$ \\
\hline $\mathrm{F}$ & 0.89073 & -1.33757 & 3.5439 \\
\hline
\end{tabular}


TSmig3 (Transition state for migration from Int. C)

\begin{tabular}{|c|c|c|c|}
\hline $\mathrm{C}$ & -4.86296 & -1.16635 & 1.76083 \\
\hline $\mathrm{C}$ & -4.46241 & -2.45730 & 2.48288 \\
\hline $\mathrm{C}$ & -3.75537 & -0.31176 & 1.26115 \\
\hline $\mathrm{C}$ & -5.12298 & -1.39189 & 0.23230 \\
\hline $\mathrm{C}$ & -3.61144 & -1.03329 & -0.01629 \\
\hline $\mathrm{H}$ & -5.79310 & -0.64670 & -0.19040 \\
\hline $\mathrm{H}$ & -5.41425 & -2.40371 & -0.04685 \\
\hline $\mathrm{H}$ & -3.02699 & -1.94955 & 0.08971 \\
\hline $\mathrm{H}$ & -3.27588 & -0.46559 & -0.88105 \\
\hline $\mathrm{H}$ & -5.60262 & -0.62132 & 2.34795 \\
\hline $\mathrm{H}$ & -3.70236 & -3.01198 & 1.93137 \\
\hline $\mathrm{H}$ & -4.07912 & -2.22993 & 3.47761 \\
\hline $\mathrm{C}$ & -2.42606 & 2.27630 & 3.39177 \\
\hline $\mathrm{C}$ & -1.36391 & 1.85523 & 2.32552 \\
\hline $\mathrm{O}$ & -3.54903 & 1.46832 & 3.03822 \\
\hline $\mathrm{O}$ & -2.16025 & 1.70879 & 1.14930 \\
\hline B & -3.45326 & 1.22803 & 1.60413 \\
\hline $\mathrm{H}$ & -5.34711 & -3.08712 & 2.58137 \\
\hline $\mathrm{C}$ & -0.28076 & 2.89020 & 2.06693 \\
\hline $\mathrm{H}$ & 0.26165 & 3.12224 & 2.98761 \\
\hline $\mathrm{H}$ & 0.43363 & 2.50069 & 1.33802 \\
\hline $\mathrm{H}$ & -0.71062 & 3.80914 & 1.66833 \\
\hline $\mathrm{C}$ & -0.73457 & 0.50039 & 2.65918 \\
\hline $\mathrm{H}$ & -0.18911 & 0.14346 & 1.78298 \\
\hline $\mathrm{H}$ & -0.03904 & 0.56848 & 3.49881 \\
\hline $\mathrm{H}$ & -1.50588 & -0.23414 & 2.91347 \\
\hline $\mathrm{C}$ & -2.81985 & 3.74734 & 3.24393 \\
\hline $\mathrm{H}$ & -3.71505 & 3.93035 & 3.84225 \\
\hline $\mathrm{H}$ & -2.02807 & 4.41686 & 3.58840 \\
\hline $\mathrm{H}$ & -3.04815 & 3.97617 & 2.20022 \\
\hline $\mathrm{C}$ & -2.02939 & 1.97704 & 4.82930 \\
\hline $\mathrm{H}$ & -1.10084 & 2.49201 & 5.09145 \\
\hline $\mathrm{H}$ & -2.81413 & 2.32443 & 5.50512 \\
\hline $\mathrm{H}$ & -1.89512 & 0.90591 & 4.98331 \\
\hline
\end{tabular}




\begin{tabular}{cccr} 
C & -4.68938 & 1.87189 & 0.72419 \\
C & -4.52187 & 2.22439 & -0.61576 \\
C & -5.93107 & 2.10758 & 1.31503 \\
C & -5.56449 & 2.79694 & -1.33780 \\
H & -3.55819 & 2.06837 & -1.09265 \\
C & -6.96924 & 2.68255 & 0.58783 \\
H & -6.07619 & 1.86117 & 2.36332 \\
C & -6.79995 & 3.02970 & -0.74584 \\
H & -7.60915 & 3.47441 & -1.30961 \\
C & -8.28291 & 2.90954 & 1.27429 \\
C & -5.32198 & 3.17734 & -2.76783 \\
F & -9.17597 & 3.52268 & 0.48769 \\
F & -8.83846 & 1.75226 & 1.67233 \\
F & -8.14366 & 3.66078 & 2.37722 \\
F & -4.83216 & 2.14789 & -3.47798 \\
F & -6.43624 & 3.58827 & -3.38607 \\
F & -4.42399 & 4.16988 & -2.87243 \\
\multicolumn{4}{c}{ G $=-1511.769232$ Hartree }
\end{tabular}

\section{Intermediate D}

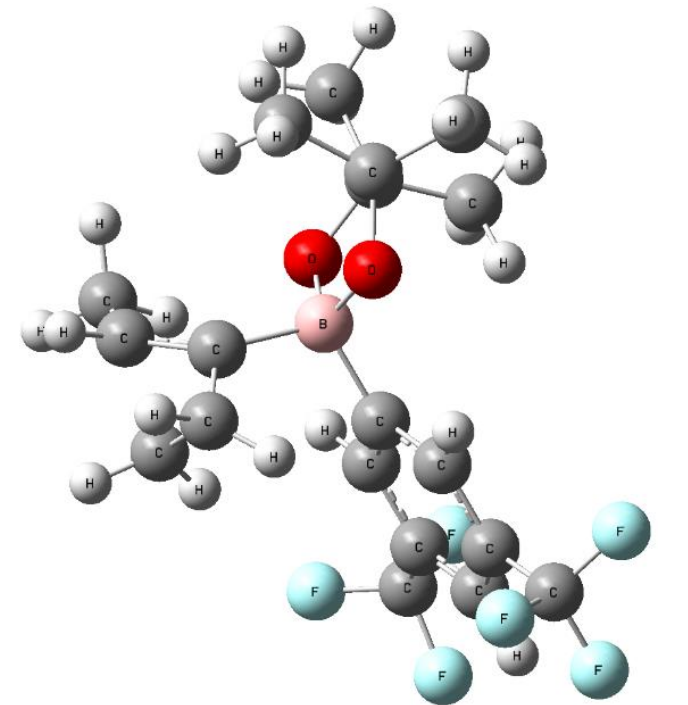

$\begin{array}{llll}\text { C } & -3.66363 & -1.10690 & -0.03669\end{array}$

$\begin{array}{llll}\text { C } & -2.31841 & -1.21462 & -0.69338\end{array}$

$\begin{array}{llll}\text { C } & -4.01491 & -0.08623 & 0.88908\end{array}$

$\begin{array}{llll}\text { C } & -3.91160 & -1.62585 & 1.56151\end{array}$

$\begin{array}{llll}\text { C } & -5.09563 & -0.60249 & 1.70915\end{array}$

$\begin{array}{llll}\mathrm{H} & -3.08394 & -1.60863 & 2.25802\end{array}$

$\begin{array}{llll}\mathrm{H} & -4.31293 & -2.61109 & 1.35994\end{array}$

$\mathrm{H} \quad-5.98656 \quad-1.01690 \quad 1.24806$

$\mathrm{H} \quad-5.24670 \quad-0.12481 \quad 2.67001$

$\begin{array}{llll}\mathrm{H} & -4.47363 & -1.61792 & -0.55597\end{array}$

$\mathrm{H} \quad-1.53628 \quad-0.79378 \quad-0.06696$

$\begin{array}{llll}\mathrm{H} & -2.10331 & -2.25886 & -0.92479\end{array}$

$\begin{array}{llll}\text { C } & -2.01182 & 2.81464 & 0.14874\end{array}$ 


\begin{tabular}{|c|c|c|c|}
\hline C & -3.54209 & 3.11902 & 0.17075 \\
\hline $\mathrm{O}$ & -1.97349 & 1.44056 & 0.52463 \\
\hline $\mathrm{O}$ & -3.98792 & 2.36303 & 1.29475 \\
\hline B & -3.09338 & 1.20830 & 1.43165 \\
\hline $\mathrm{H}$ & -2.33308 & -0.65495 & -1.63180 \\
\hline $\mathrm{C}$ & -3.89326 & 4.58360 & 0.38947 \\
\hline $\mathrm{H}$ & -3.45841 & 5.20901 & -0.39529 \\
\hline $\mathrm{H}$ & -4.97828 & 4.70798 & 0.36326 \\
\hline $\mathrm{H}$ & -3.53264 & 4.93026 & 1.35803 \\
\hline $\mathrm{C}$ & -4.24393 & 2.59688 & -1.0861 \\
\hline $\mathrm{H}$ & -5.32247 & 2.60987 & -0.9148 \\
\hline $\mathrm{H}$ & -4.02245 & 3.21255 & -1.96132 \\
\hline $\mathrm{H}$ & -3.93804 & 1.56929 & -1.30143 \\
\hline $\mathrm{C}$ & -1.25468 & 3.63514 & 1.19630 \\
\hline $\mathrm{H}$ & -0.25057 & 3.21868 & 1.30356 \\
\hline $\mathrm{H}$ & -1.16854 & 4.68498 & 0.90546 \\
\hline $\mathrm{H}$ & -1.75595 & 3.57688 & 2.16544 \\
\hline $\mathrm{C}$ & -1.35492 & 2.97182 & -1.21472 \\
\hline $\mathrm{H}$ & -1.47756 & 3.99035 & -1.5936 \\
\hline $\mathrm{H}$ & -0.28537 & 2.76617 & -1.1302 \\
\hline $\mathrm{H}$ & -1.78116 & 2.27274 & -1.93509 \\
\hline $\mathrm{C}$ & -2.65249 & 0.97544 & 2.97684 \\
\hline $\mathrm{C}$ & -1.46160 & 0.31555 & 3.29290 \\
\hline $\mathrm{C}$ & -3.42461 & 1.44162 & 4.04261 \\
\hline $\mathrm{C}$ & -1.07328 & 0.11208 & 4.61356 \\
\hline $\mathrm{H}$ & -0.82150 & -0.03761 & 2.4882 \\
\hline $\mathrm{C}$ & -3.03513 & 1.23546 & 5.3641 \\
\hline $\mathrm{H}$ & -4.33804 & 1.99148 & 3.8320 \\
\hline $\mathrm{C}$ & -1.85681 & 0.56713 & 5.66697 \\
\hline $\mathrm{H}$ & -1.55450 & 0.40730 & $6.6933^{\prime}$ \\
\hline $\mathrm{C}$ & -3.92353 & 1.74577 & 6.45761 \\
\hline $\mathrm{C}$ & 0.23038 & -0.57565 & 4.88270 \\
\hline $\mathrm{F}$ & -5.13400 & 1.16043 & 6.42706 \\
\hline $\mathrm{F}$ & -3.41746 & 1.53017 & 7.67882 \\
\hline $\mathrm{F}$ & -4.14247 & 3.06597 & 6.3479 \\
\hline $\mathrm{F}$ & 1.28027 & 0.20762 & 4.58312 \\
\hline $\mathrm{F}$ & 0.36774 & -0.93531 & 6.16614 \\
\hline $\mathrm{F}$ & 0.36855 & -1.68895 & 4.1433 \\
\hline \multicolumn{4}{|c|}{$G=-1511.781346$ Hartree } \\
\hline
\end{tabular}


TSmig4 (Transition state for migration from Int. D)

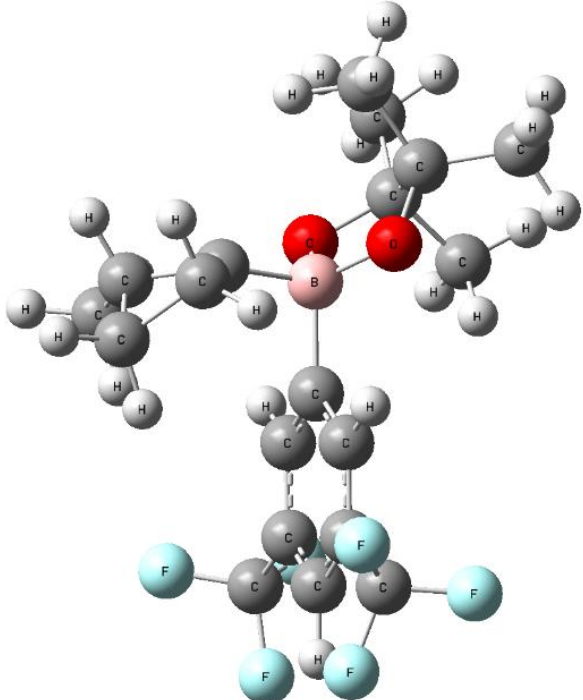

$\begin{array}{lrrr}\text { C } & -4.71584 & -1.29630 & 1.63380 \\ \mathrm{C} & -5.83290 & -0.92821 & 2.58888 \\ \mathrm{C} & -3.68365 & -0.32127 & 1.21793 \\ \mathrm{C} & -4.90650 & -1.47817 & 0.07978 \\ \mathrm{C} & -3.43214 & -0.99315 & -0.07960 \\ \mathrm{H} & -5.61366 & -0.74946 & -0.31425 \\ \mathrm{H} & -5.13602 & -2.48388 & -0.26347 \\ \mathrm{H} & -2.76101 & -1.84719 & 0.03979 \\ \mathrm{H} & -3.10951 & -0.37408 & -0.91294 \\ \mathrm{H} & -4.18678 & -2.20250 & 1.95526 \\ \mathrm{H} & -6.53575 & -0.24454 & 2.11094 \\ \mathrm{H} & -6.38004 & -1.82002 & 2.89522 \\ \mathrm{C} & -2.46669 & 2.27025 & 3.42757 \\ \mathrm{C} & -1.38032 & 1.90735 & 2.36461 \\ \mathrm{O} & -3.55567 & 1.42588 & 3.05036 \\ \mathrm{O} & -2.15983 & 1.74639 & 1.17970 \\ \mathrm{~B} & -3.44581 & 1.22240 & 1.61078 \\ \mathrm{H} & -5.42313 & -0.43830 & 3.47364 \\ \mathrm{C} & -0.33860 & 2.98970 & 2.12922 \\ \mathrm{H} & 0.19122 & 3.22742 & 3.05577 \\ \mathrm{H} & 0.39279 & 2.64059 & 1.39671 \\ \mathrm{H} & -0.80307 & 3.89734 & 1.74402 \\ \mathrm{C} & -0.69756 & 0.57541 & 2.68460 \\ \mathrm{H} & -0.12890 & 0.25529 & 1.80890 \\ \mathrm{H} & -0.01366 & 0.65977 & 3.53227 \\ \mathrm{H} & -1.43991 & -0.19385 & 2.91938 \\ \mathrm{C} & -2.91387 & 3.72820 & 3.30199 \\ \mathrm{H} & -3.81843 & 3.86862 & 3.89788 \\ \mathrm{H} & -2.14907 & 4.42002 & 3.66319 \\ \mathrm{H} & -3.14437 & 3.96718 & 2.26108 \\ \mathrm{C} & -2.07176 & 1.96133 & 4.86351 \\ \mathrm{H} & -1.16702 & 2.50839 & 5.14347\end{array}$




$\begin{array}{lllr}\text { H } & -2.87610 & 2.26505 & 5.53731 \\ \mathrm{H} & -1.89565 & 0.89400 & 5.00028 \\ \mathrm{C} & -4.68723 & 1.86374 & 0.73690 \\ \mathrm{C} & -4.57806 & 2.11999 & -0.63259 \\ \mathrm{C} & -5.88166 & 2.20421 & 1.37098 \\ \mathrm{C} & -5.63450 & 2.68105 & -1.34121 \\ \mathrm{H} & -3.65154 & 1.89322 & -1.15091 \\ \mathrm{C} & -6.93800 & 2.76183 & 0.65547 \\ \mathrm{H} & -5.97386 & 2.04256 & 2.44093 \\ \mathrm{C} & -6.83037 & 3.00048 & -0.70705 \\ \mathrm{H} & -7.65673 & 3.42417 & -1.26291 \\ \mathrm{C} & -8.21179 & 3.06237 & 1.38609 \\ \mathrm{C} & -5.46407 & 2.98354 & -2.80003 \\ \mathrm{~F} & -9.11171 & 3.68823 & 0.61794 \\ \mathrm{~F} & -8.79546 & 1.93693 & 1.83691 \\ \mathrm{~F} & -8.00084 & 3.83430 & 2.46236 \\ \mathrm{~F} & -6.63737 & 3.03997 & -3.44537 \\ \mathrm{~F} & -4.85528 & 4.16412 & -2.99908 \\ \mathrm{~F} & -4.71407 & 2.05894 & -3.41823 \\ & \mathrm{G}=-1511.771631 \text { Hartree }\end{array}$


6. NMR spectra

${ }^{1} \mathbf{H}-\mathrm{NMR}\left(400 \mathrm{MHz}, \mathrm{CDCl}_{3}\right)$ of compound $4 \mathbf{a}$ see procedure

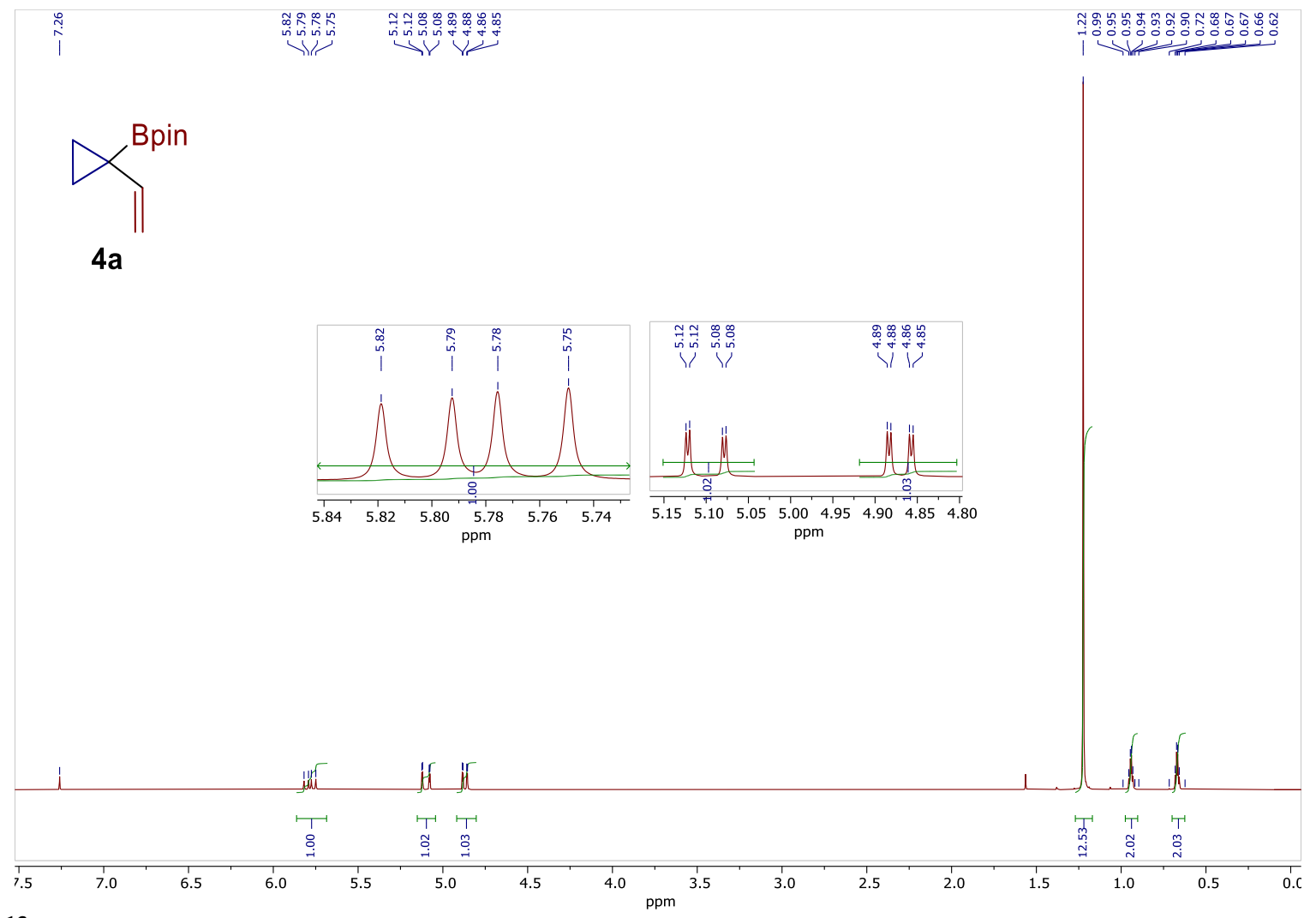

${ }^{13} \mathbf{C}$-NMR (100 MHz, $\left.\mathrm{CDCl}_{3}\right)$ of compound $\mathbf{4 a}$

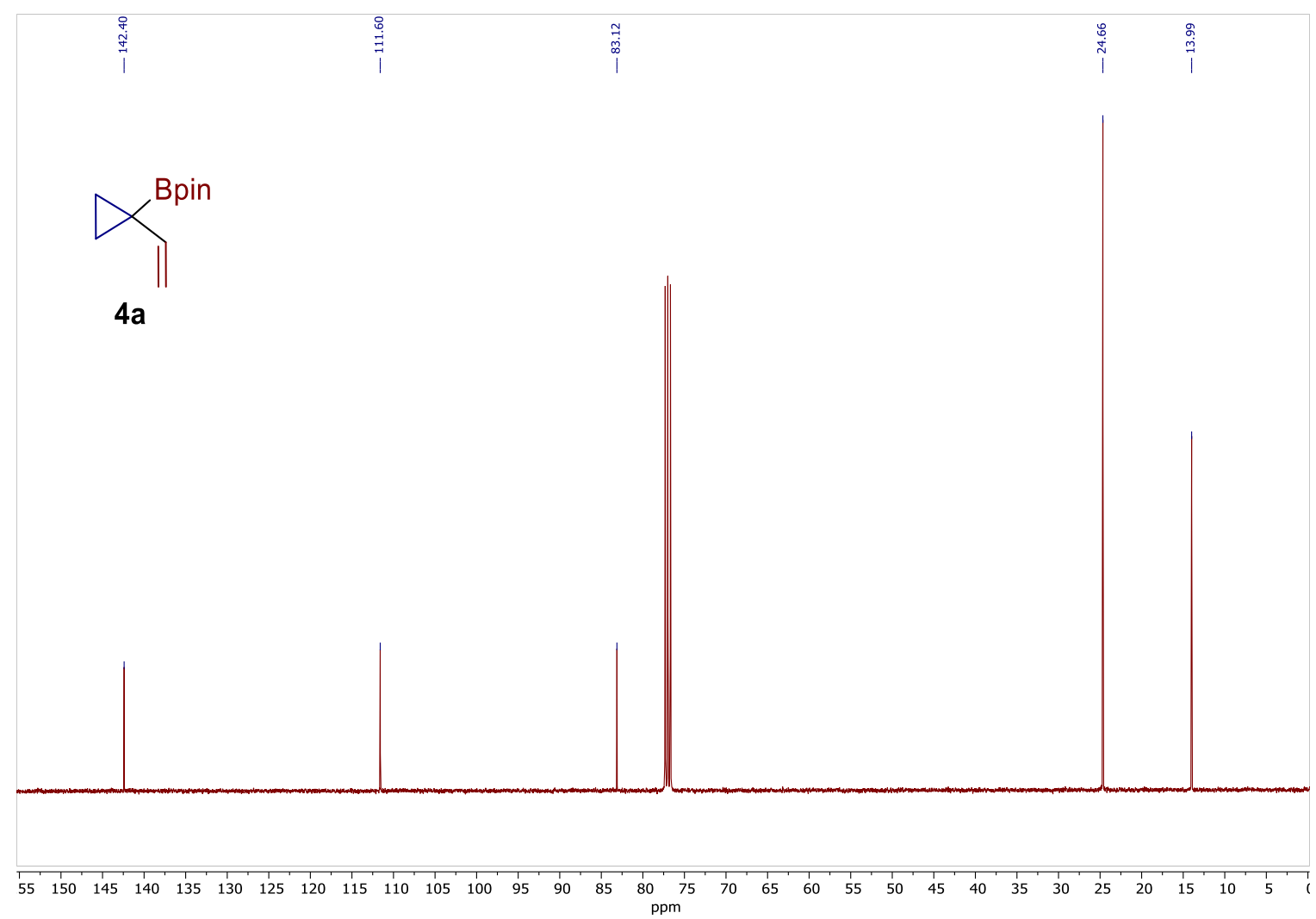


${ }^{1} \mathbf{H}-\mathrm{NMR}\left(400 \mathrm{MHz}, \mathrm{CDCl}_{3}\right)$ of compound $\mathbf{4 b}$ see procedure

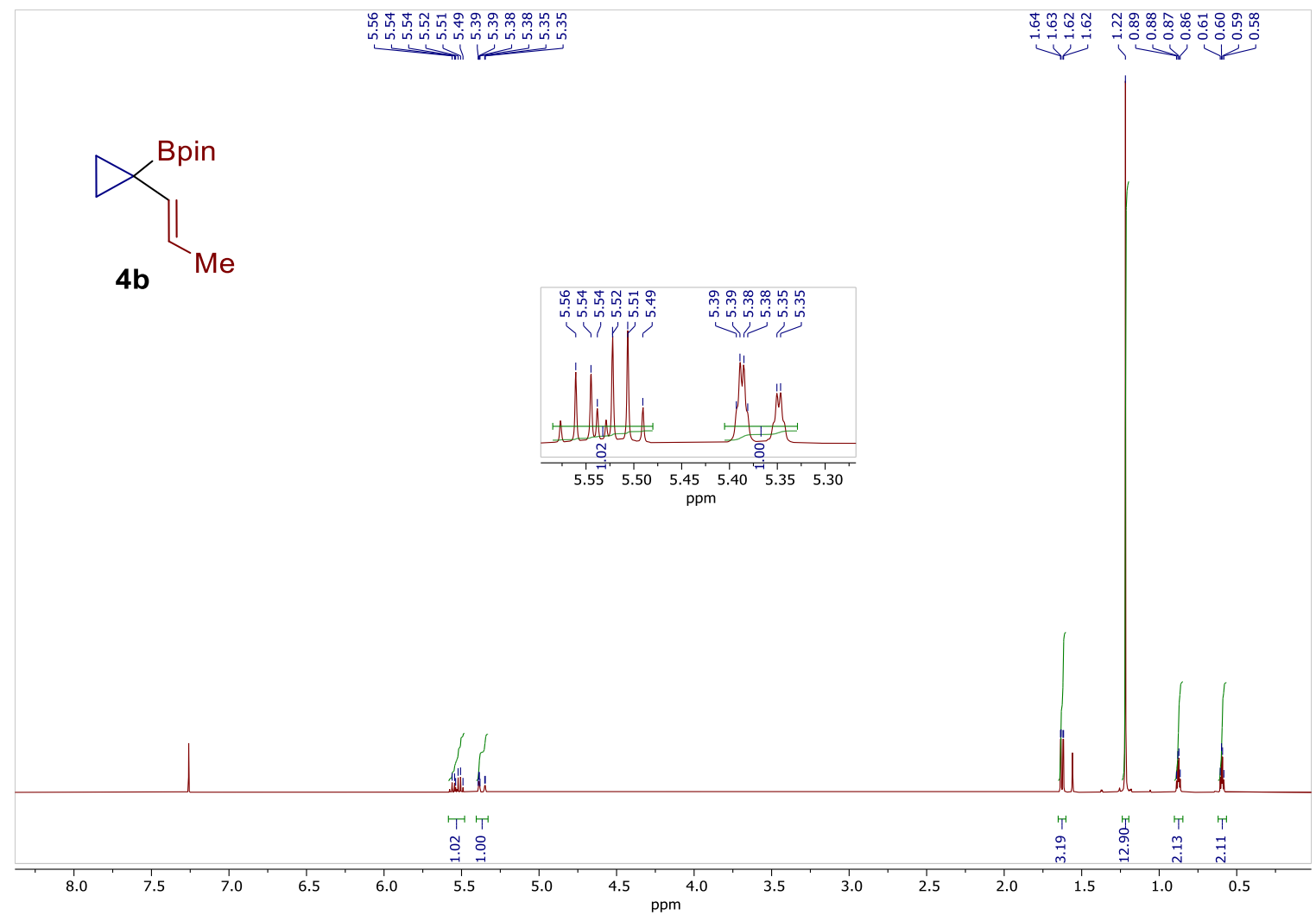

${ }^{13} \mathbf{C}$-NMR $\left(100 \mathrm{MHz}, \mathrm{CDCl}_{3}\right)$ of compound $\mathbf{4 b}$

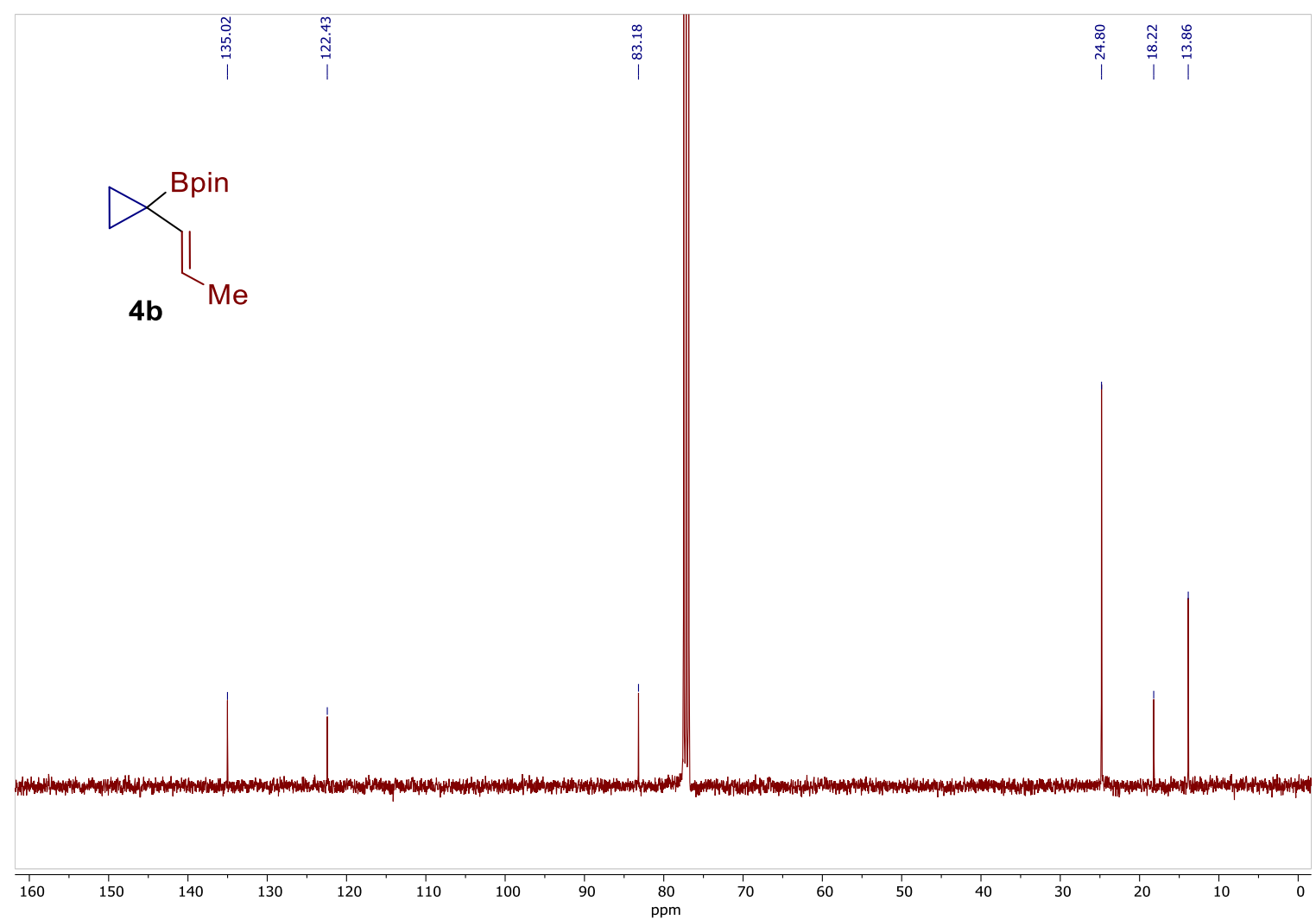


${ }^{1} \mathbf{H}-\mathrm{NMR}\left(400 \mathrm{MHz}, \mathrm{CDCl}_{3}\right)$ of compound $\mathbf{4 c}$ see procedure

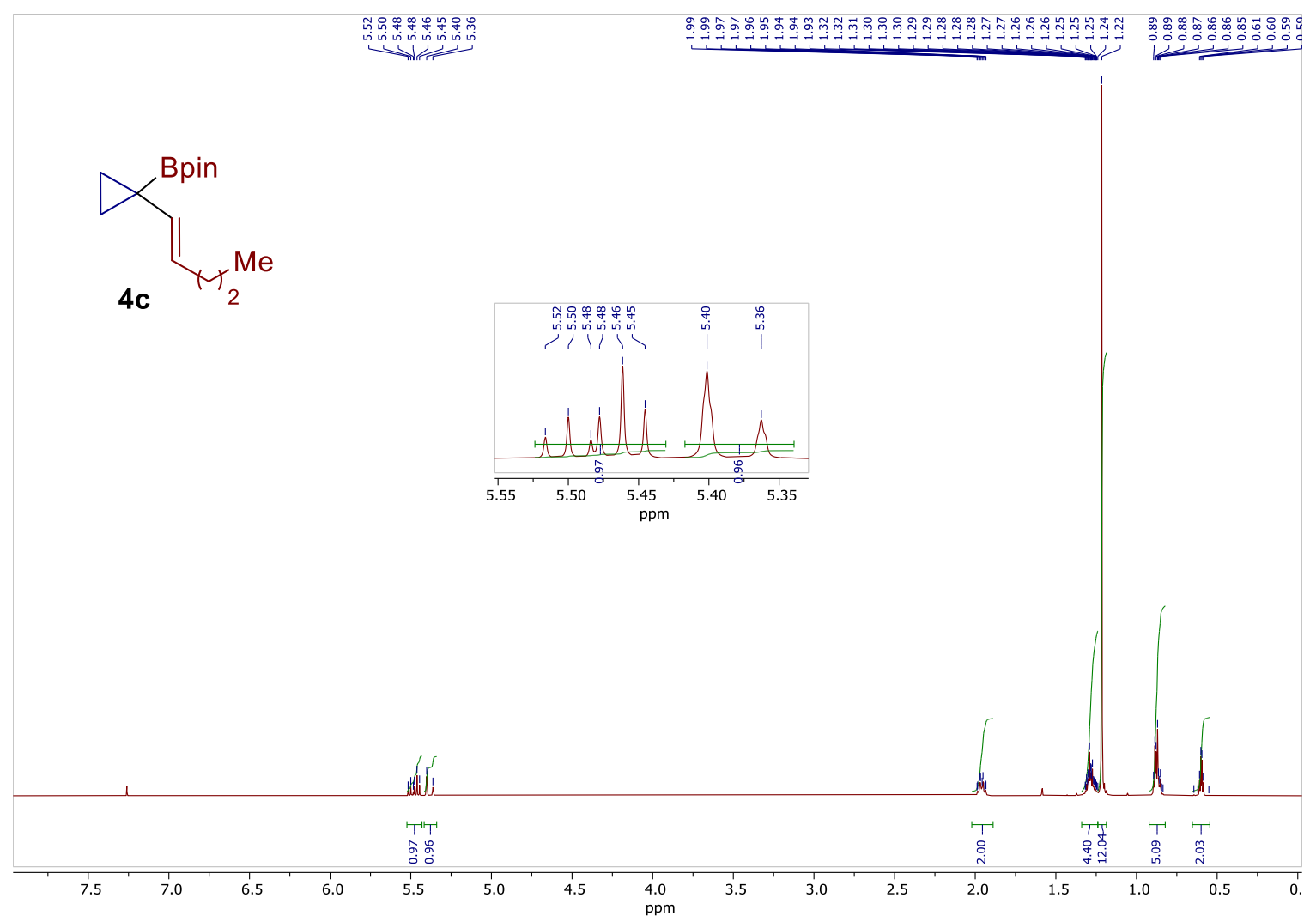

${ }^{13} \mathrm{C}-\mathrm{NMR}\left(100 \mathrm{MHz}, \mathrm{CDCl}_{3}\right)$ of compound $\mathbf{4 c}$

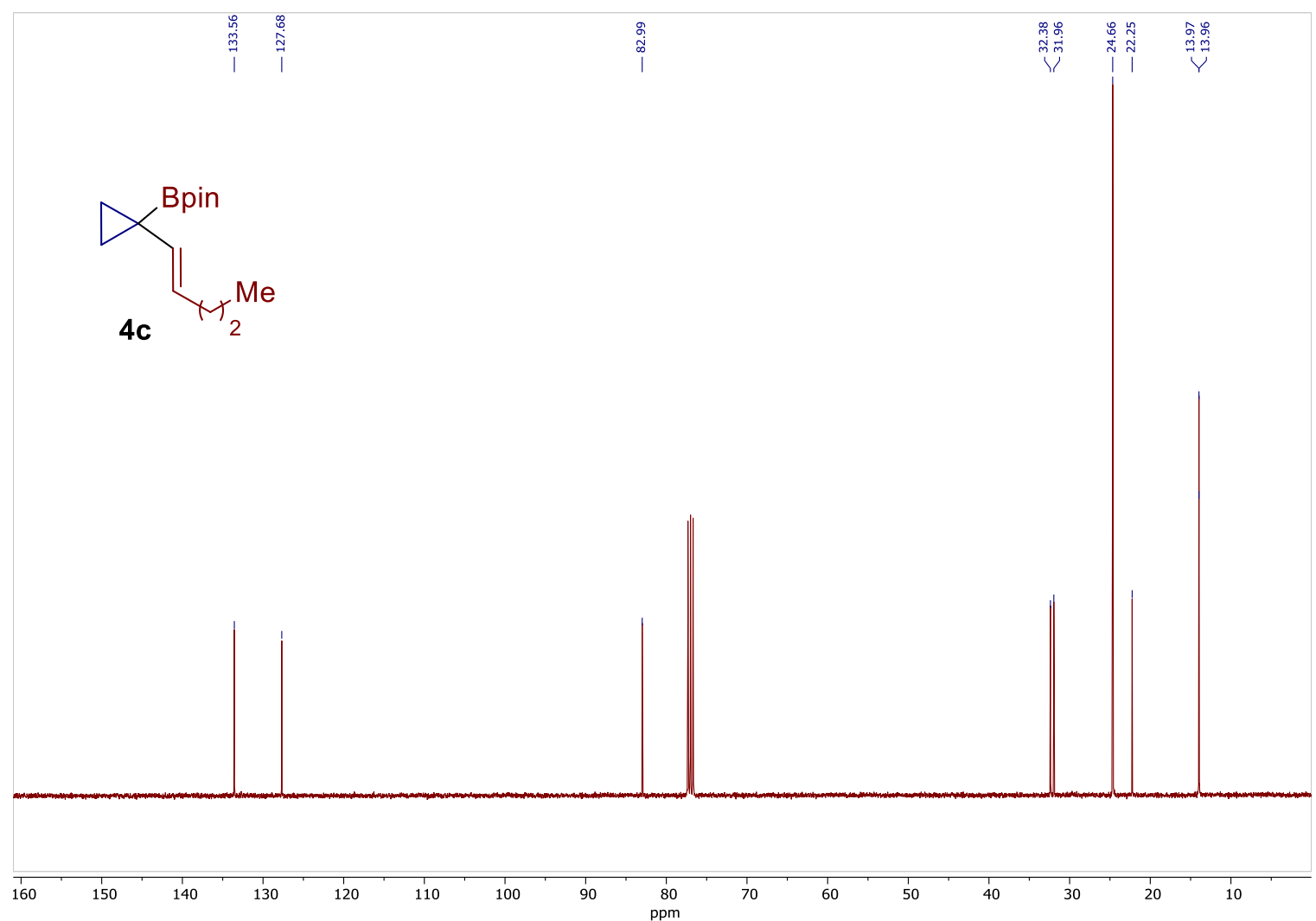


${ }^{1} \mathbf{H}-\mathrm{NMR}\left(400 \mathrm{MHz}, \mathrm{CDCl}_{3}\right)$ of compound $\mathbf{4 d}$ see procedure

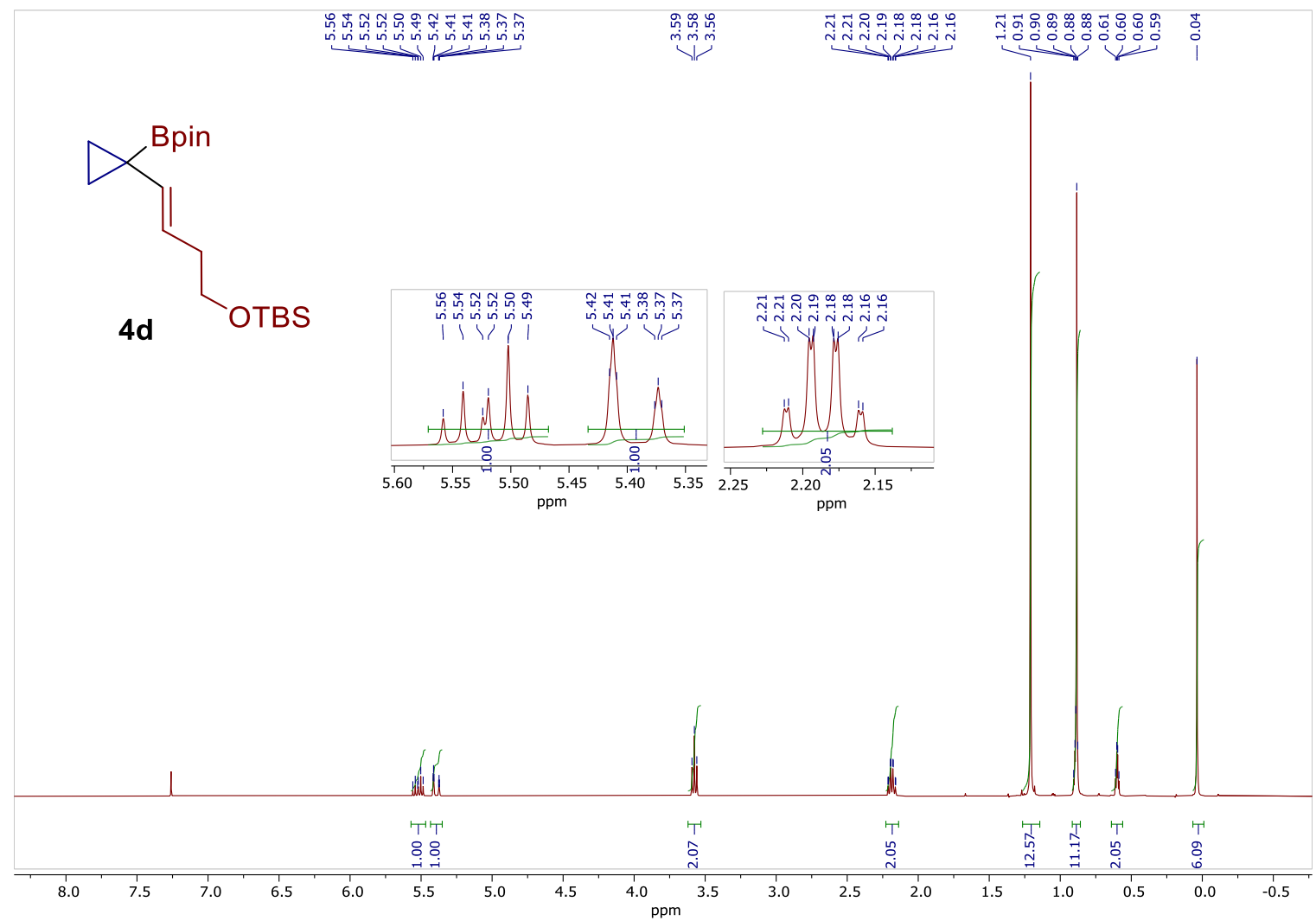

${ }^{13} \mathbf{C}$-NMR (100 MHz, $\left.\mathrm{CDCl}_{3}\right)$ of compound $\mathbf{4 d}$

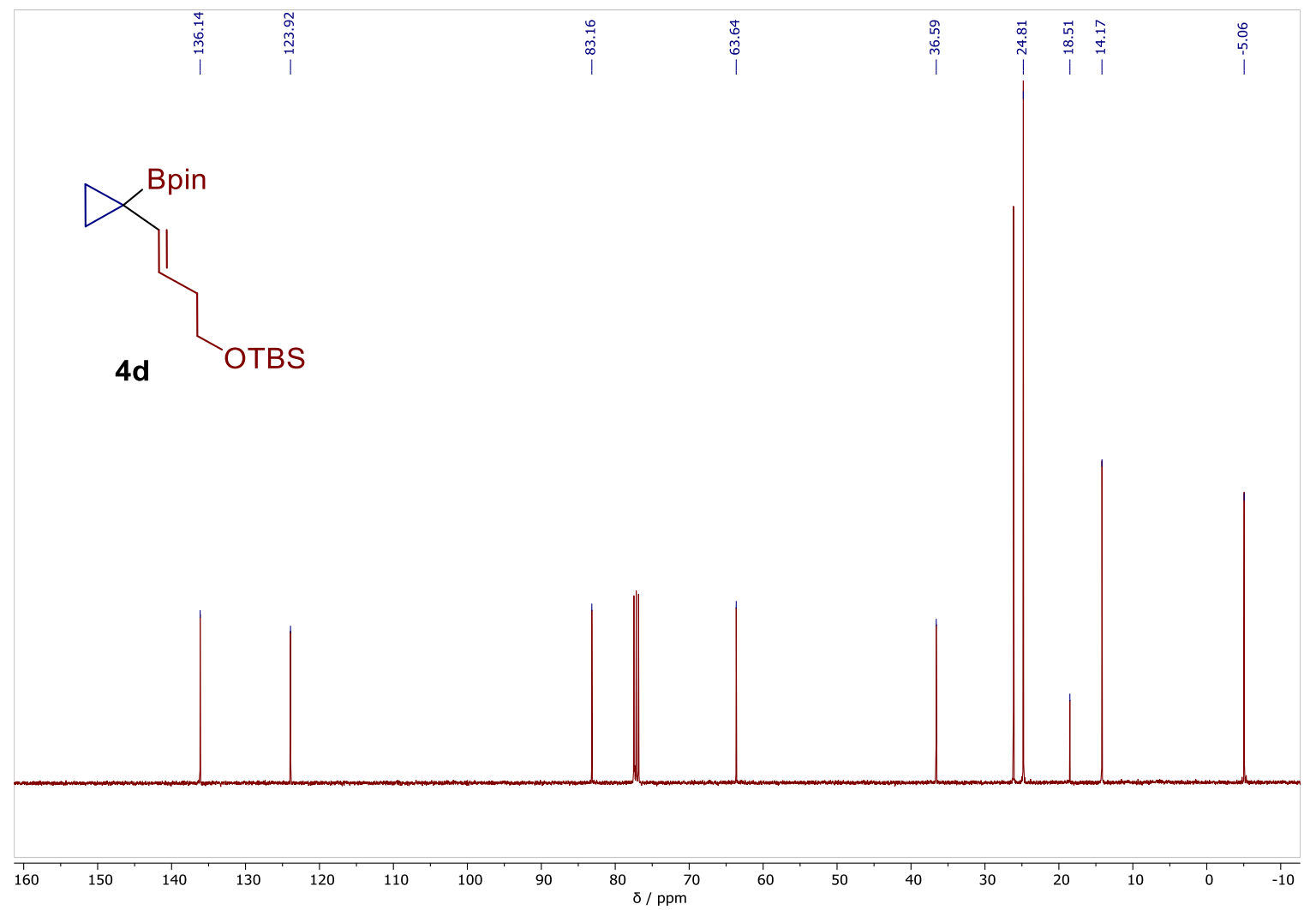


${ }^{1} \mathbf{H}-\mathrm{NMR}\left(400 \mathrm{MHz}, \mathrm{CDCl}_{3}\right)$ of compound $4 \mathbf{e}$ see procedure

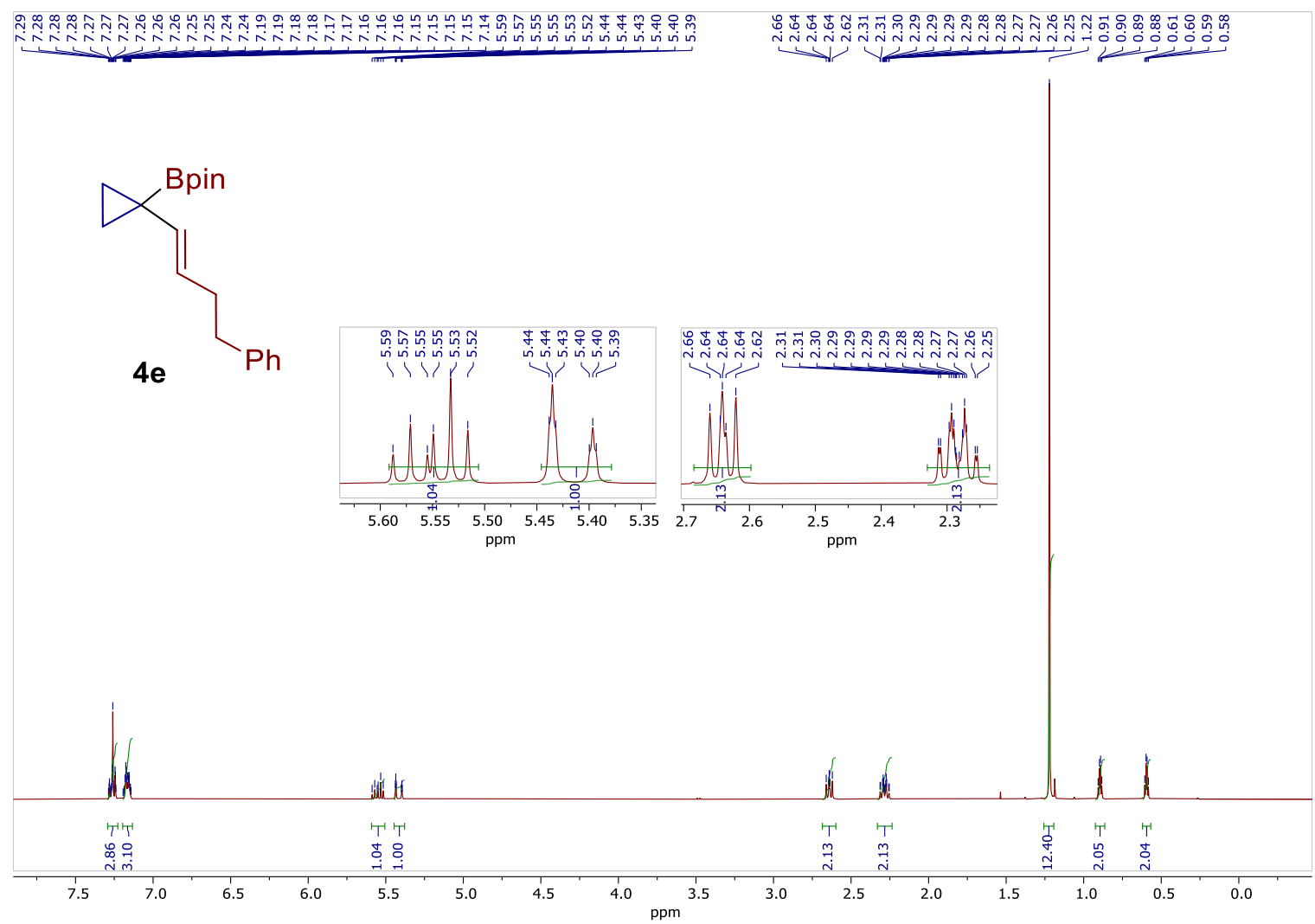

${ }^{13} \mathbf{C}$-NMR (100 MHz, $\left.\mathrm{CDCl}_{3}\right)$ of compound $\mathbf{4 e}$

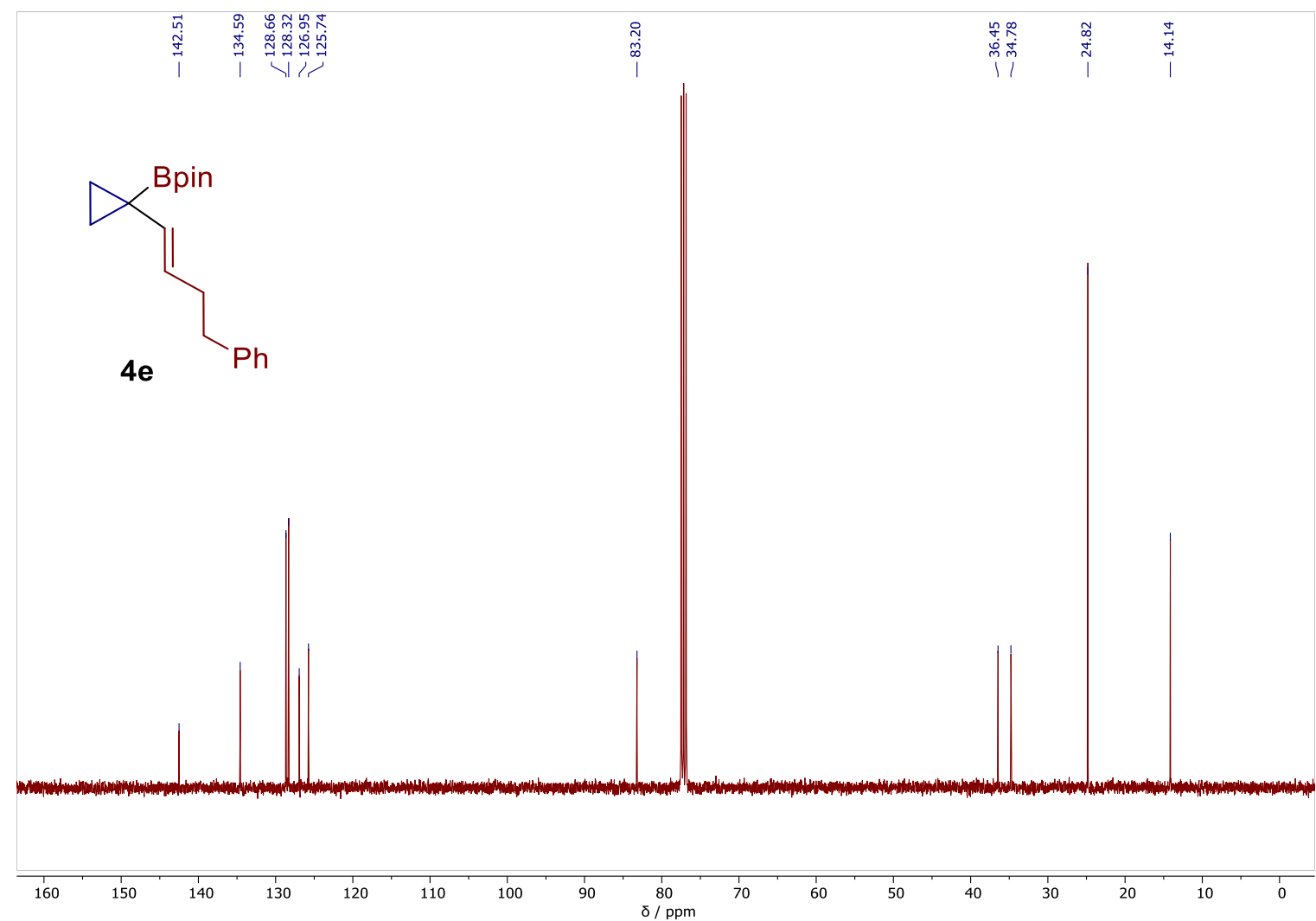


${ }^{1} \mathbf{H}-\mathrm{NMR}\left(400 \mathrm{MHz}, \mathrm{CDCl}_{3}\right)$ of compound $\mathbf{4 f}$ see procedure

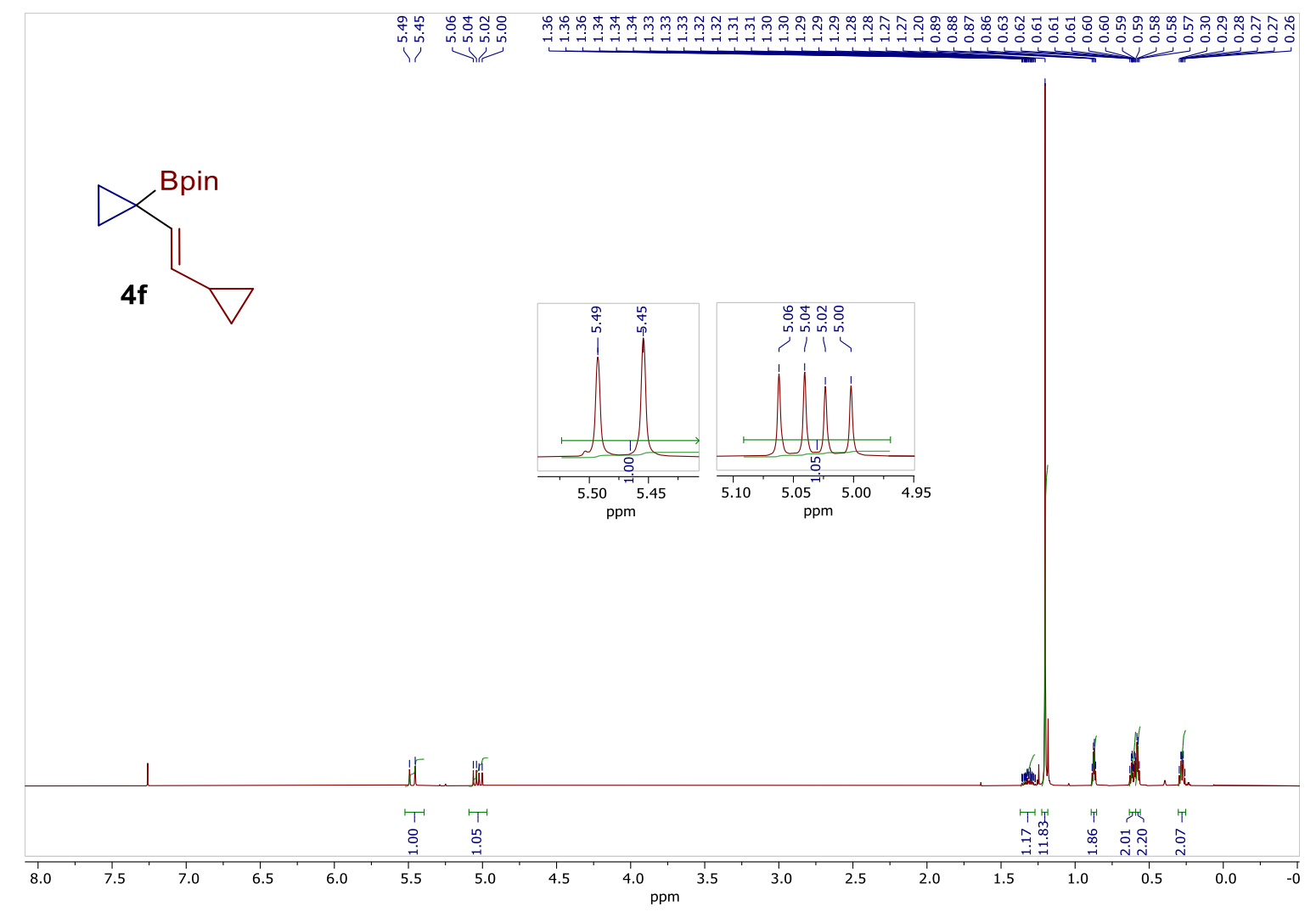

${ }^{13} \mathbf{C}$-NMR $\left(100 \mathrm{MHz}, \mathrm{CDCl}_{3}\right)$ of compound $\mathbf{4 f}$

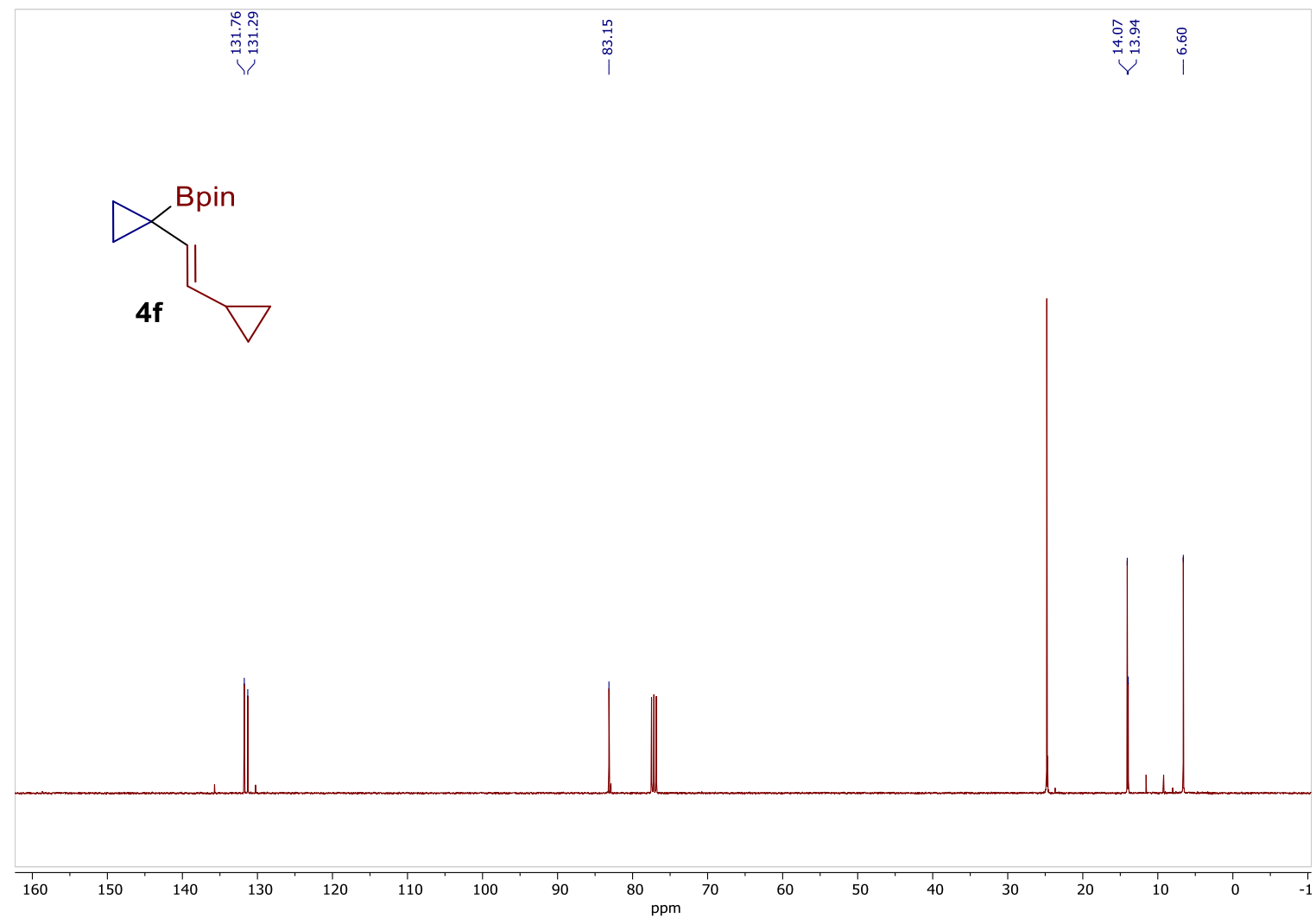


${ }^{\mathbf{1}} \mathbf{H}-\mathrm{NMR}\left(400 \mathrm{MHz}, \mathrm{CDCl}_{3}\right)$ of compound $\mathbf{4 g}$ see procedure

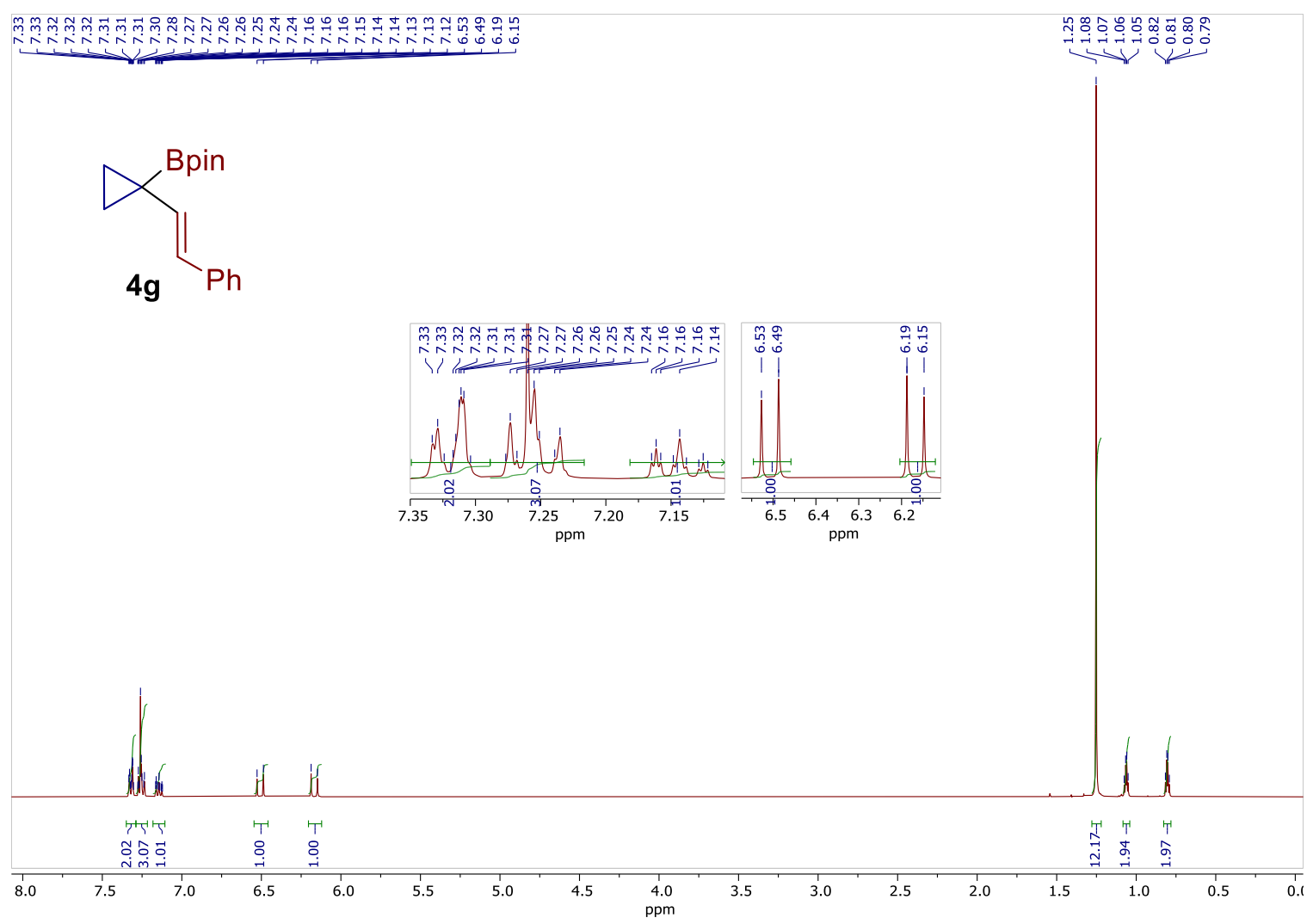

${ }^{13} \mathbf{C}-\mathbf{N M R}\left(100 \mathrm{MHz}, \mathrm{CDCl}_{3}\right)$ of compound $\mathbf{4 g}$

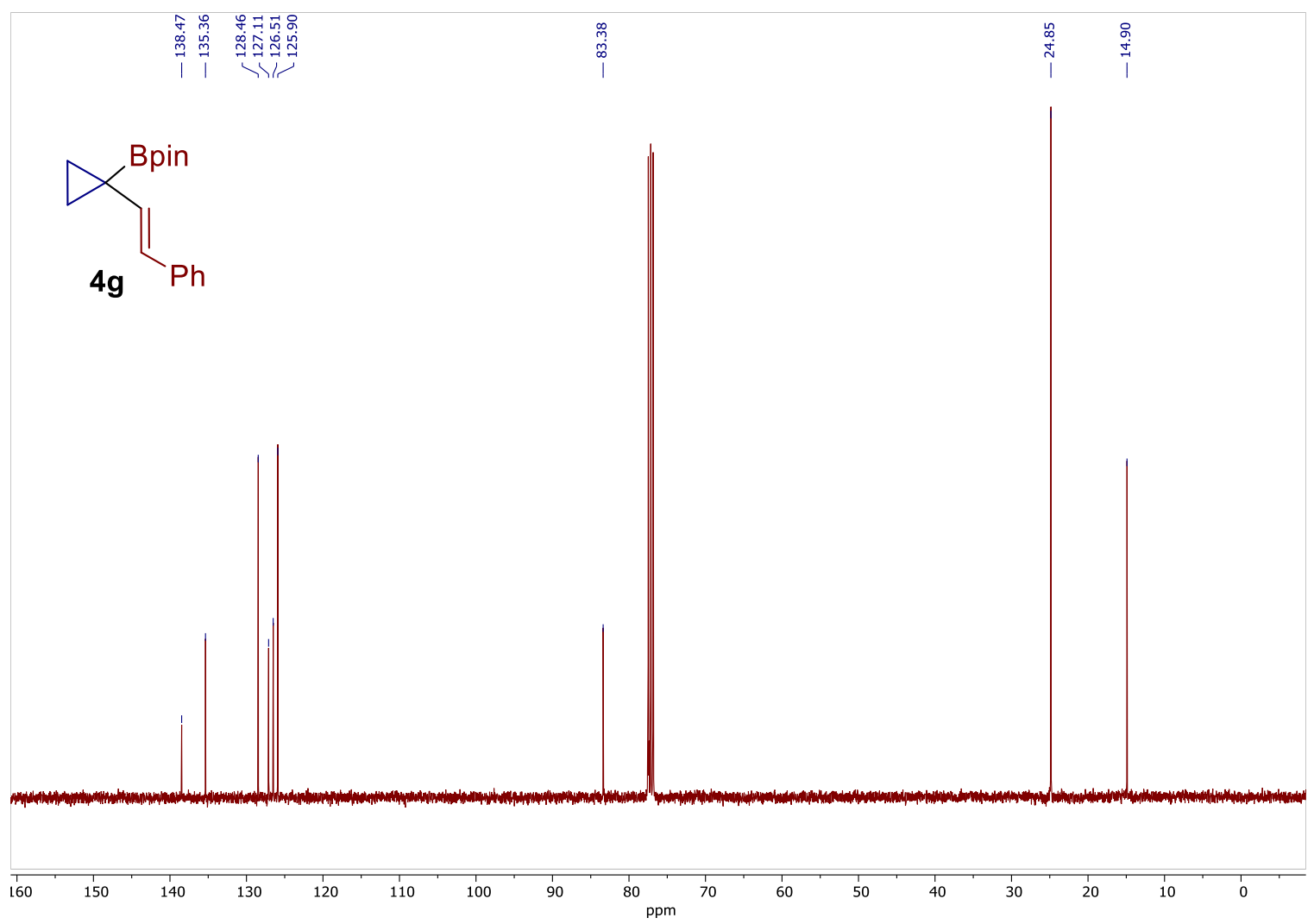

S67 
${ }^{1} \mathbf{H}-\mathrm{NMR}\left(400 \mathrm{MHz}, \mathrm{CDCl}_{3}\right)$ of compound $\mathbf{4 h}$ see procedure

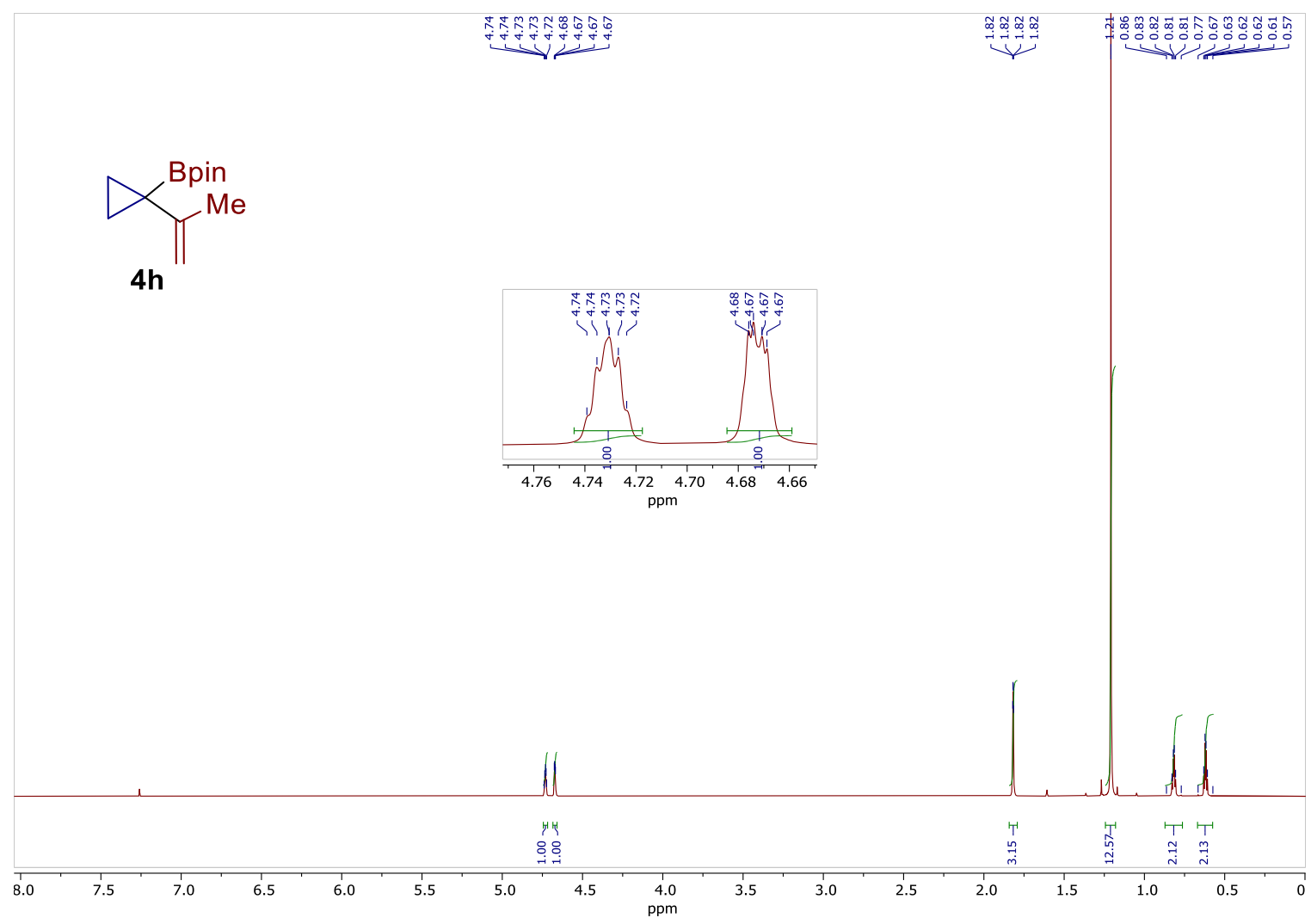

${ }^{13} \mathbf{C}$-NMR (100 MHz, $\left.\mathrm{CDCl}_{3}\right)$ of compound $\mathbf{4 h}$

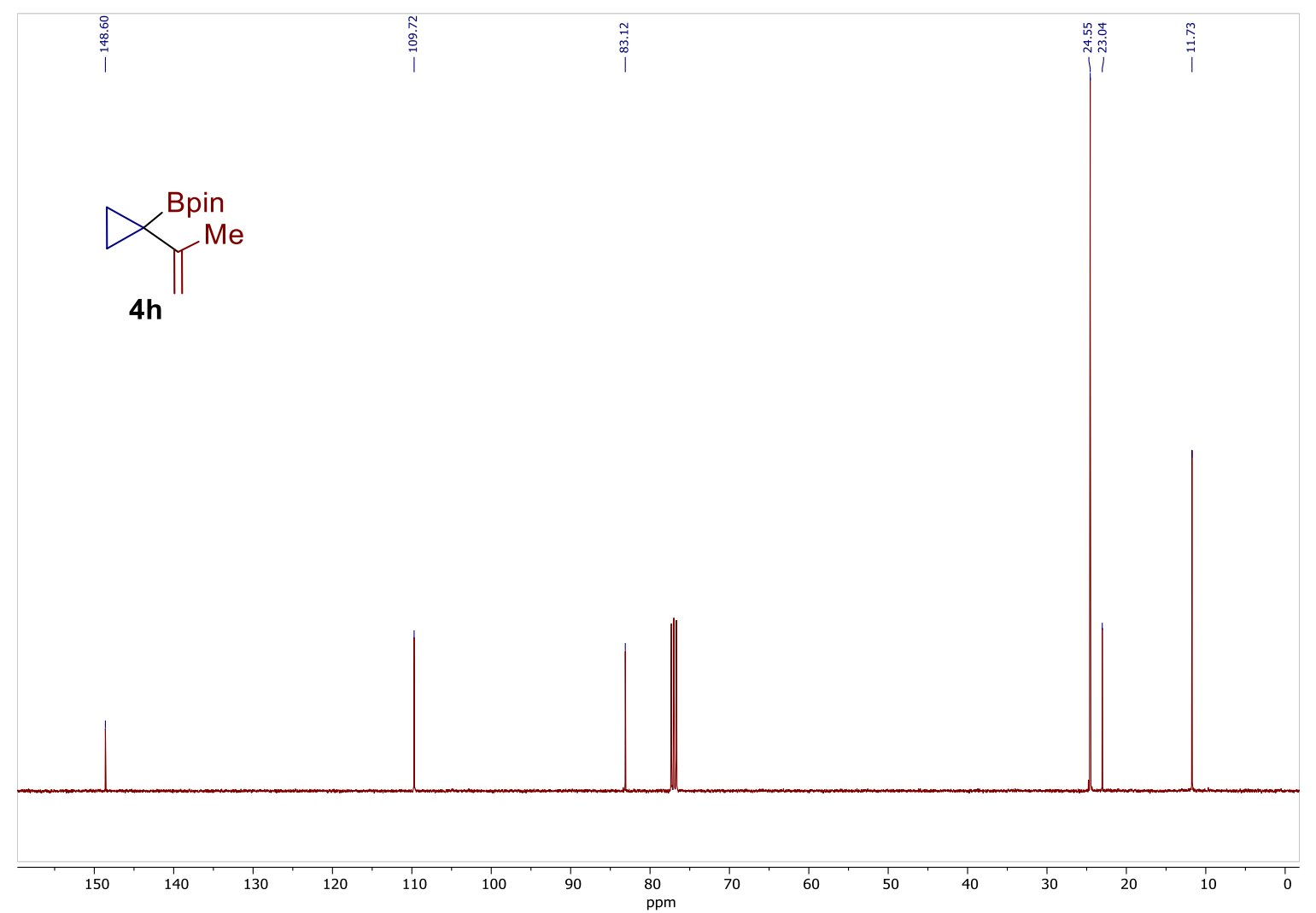


${ }^{1} \mathbf{H}-\mathbf{N M R}\left(400 \mathrm{MHz}, \mathrm{CDCl}_{3}\right)$ of compound $4 \mathbf{i}$ see procedure

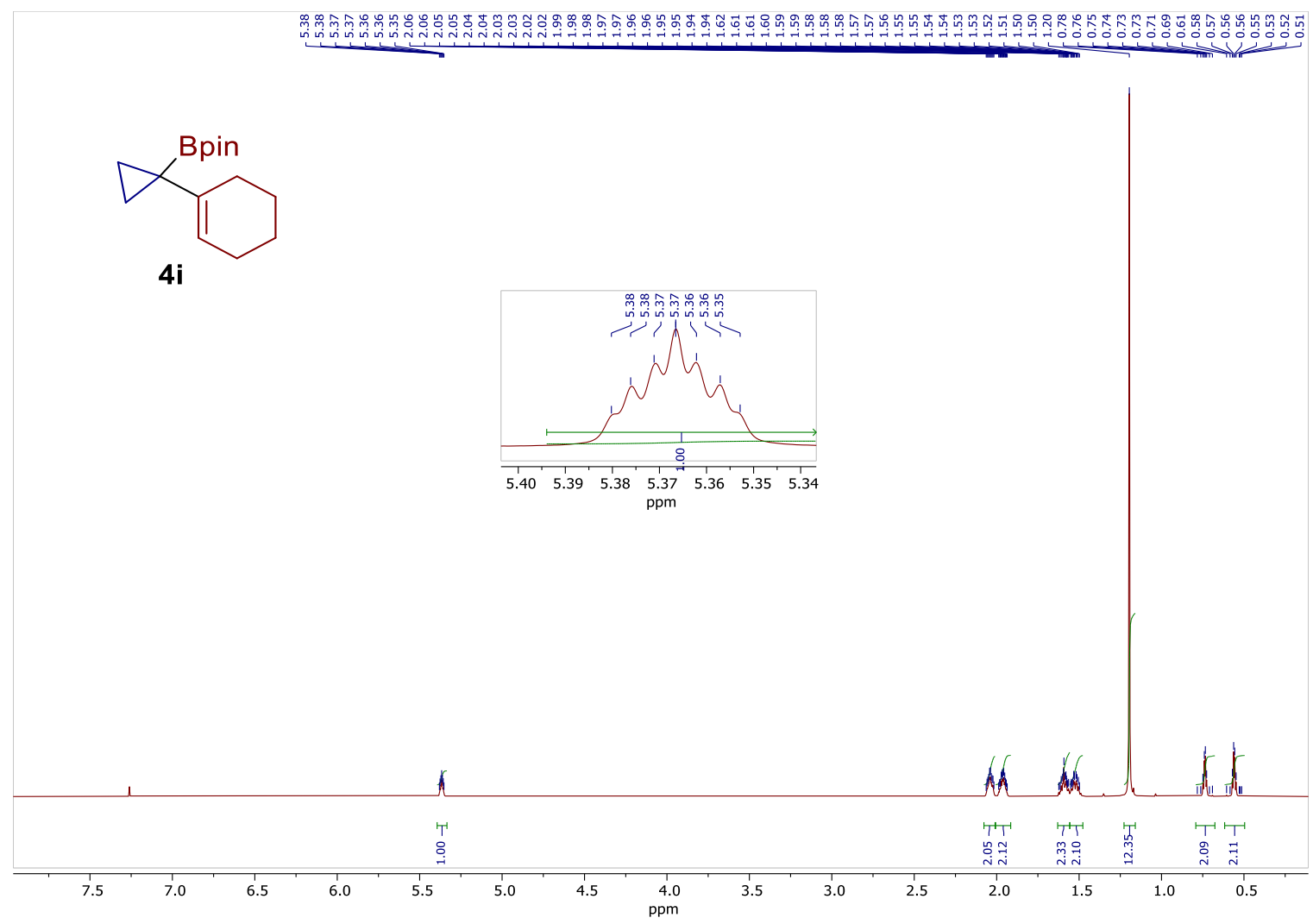

${ }^{13} \mathbf{C}-\mathbf{N M R}\left(100 \mathrm{MHz}, \mathrm{CDCl}_{3}\right)$ of compound $\mathbf{4 i}$

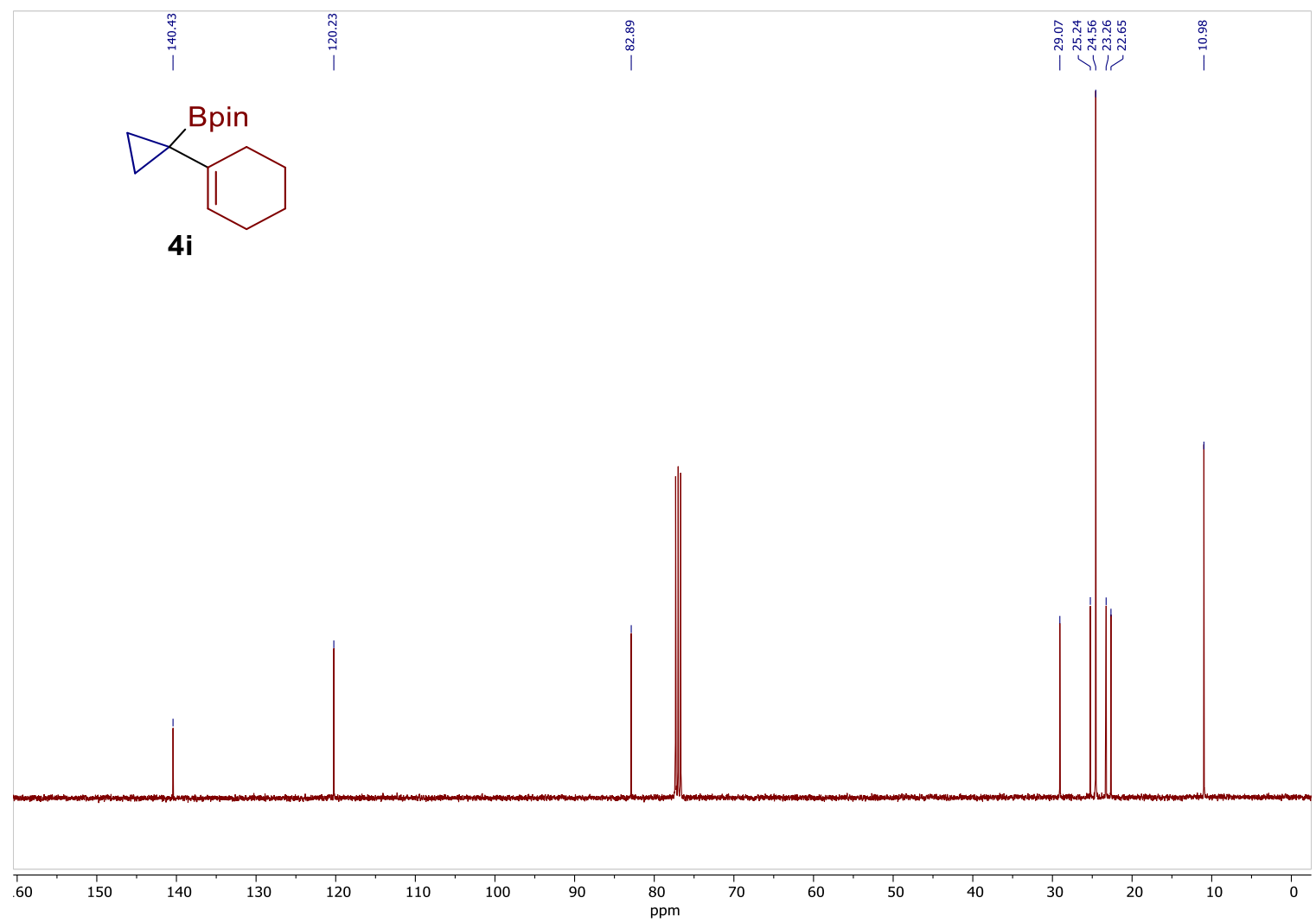


1H-NMR (400 MHz, CDCl3) of compound 1a see procedure

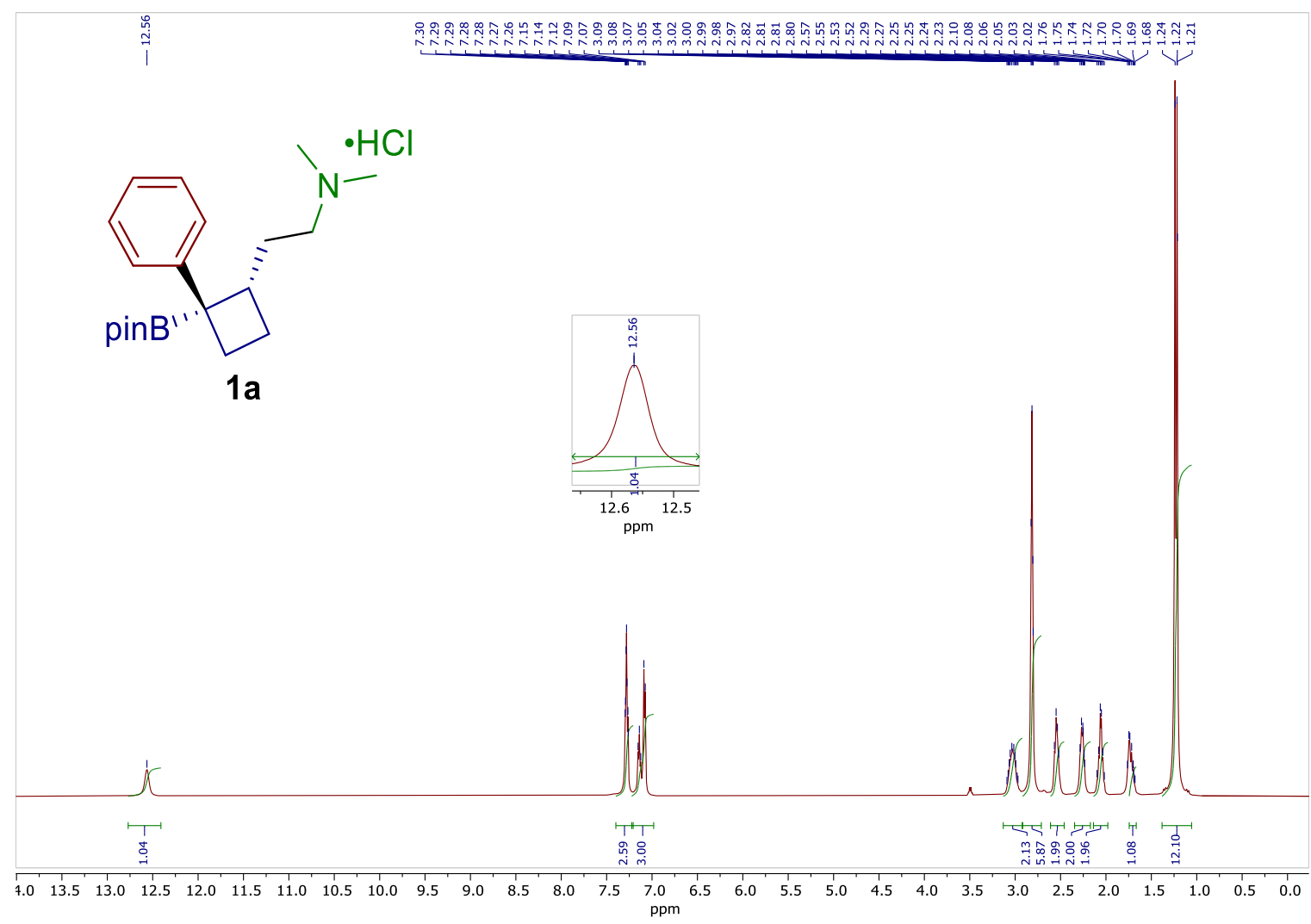

${ }^{13} \mathbf{C}-\mathbf{N M R}\left(100 \mathrm{MHz}, \mathrm{CDCl}_{3}\right)$ of compound $\mathbf{1 a}$

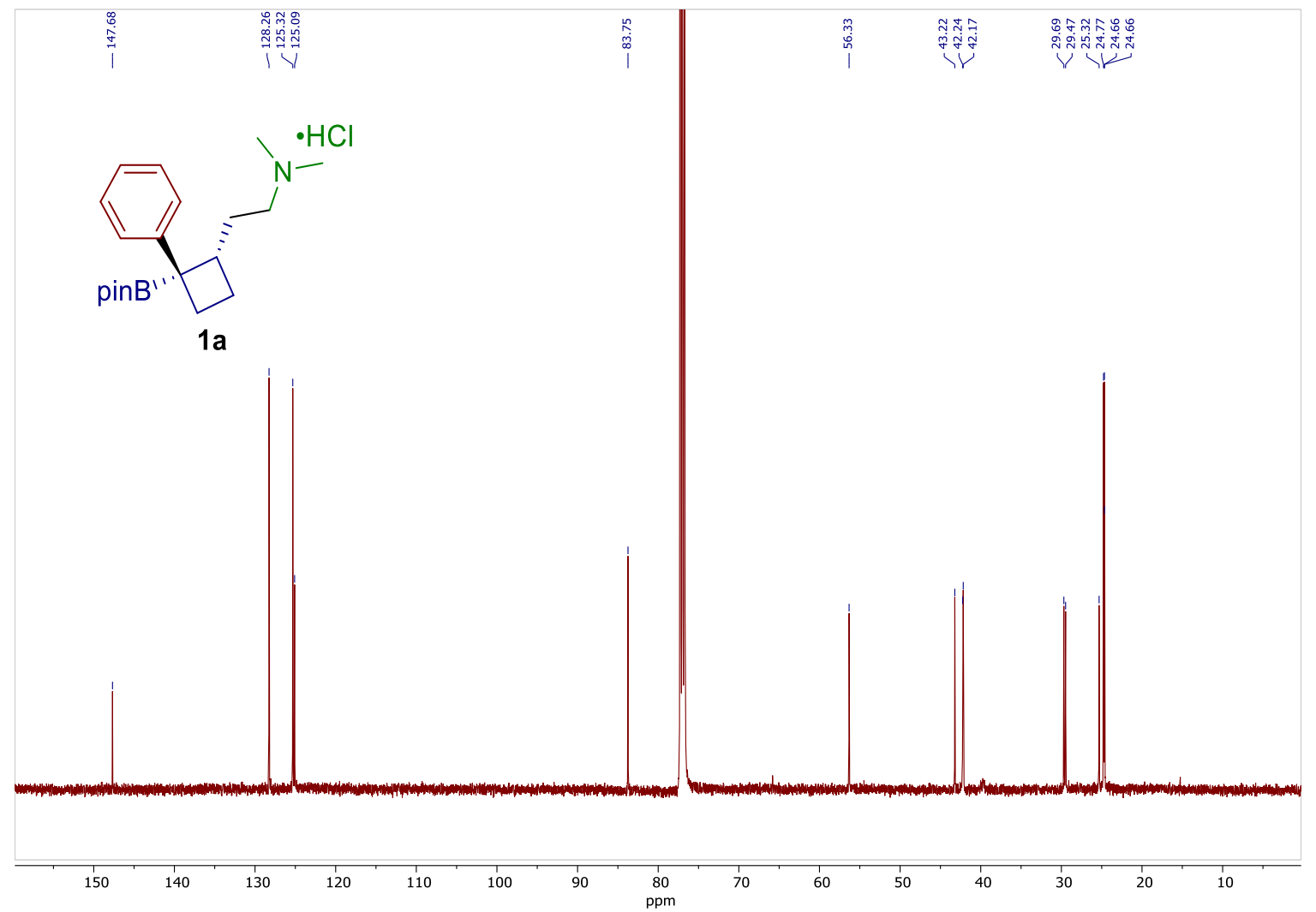


${ }^{\mathbf{1}} \mathbf{H}-\mathbf{N M R}(400 \mathrm{MHz}, \mathrm{CDCl} 3)$ of compound $\mathbf{1 b}$ see procedure

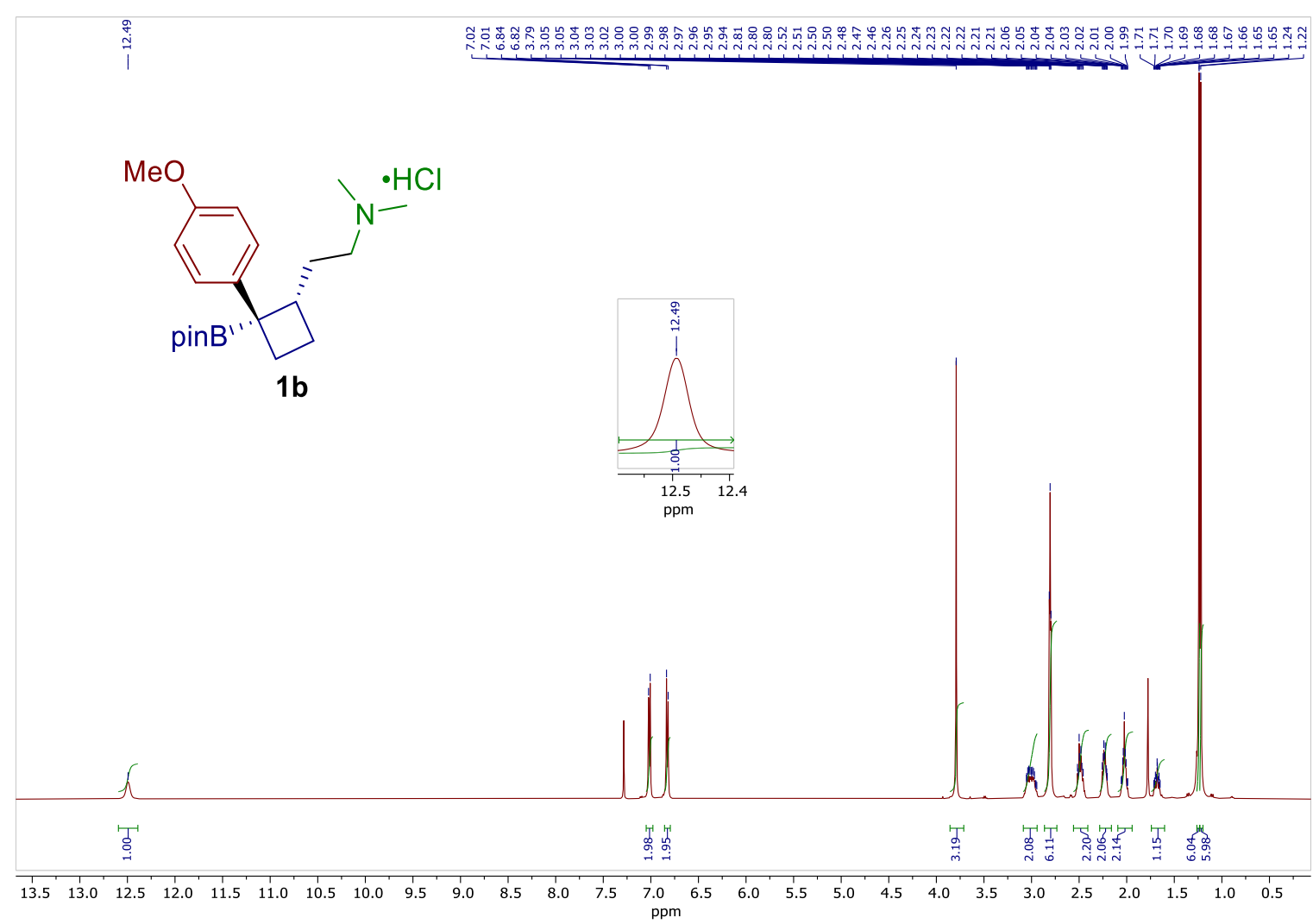

${ }^{13} \mathbf{C}$-NMR $\left(100 \mathrm{MHz}, \mathrm{CDCl}_{3}\right)$ of compound $\mathbf{1 b}$

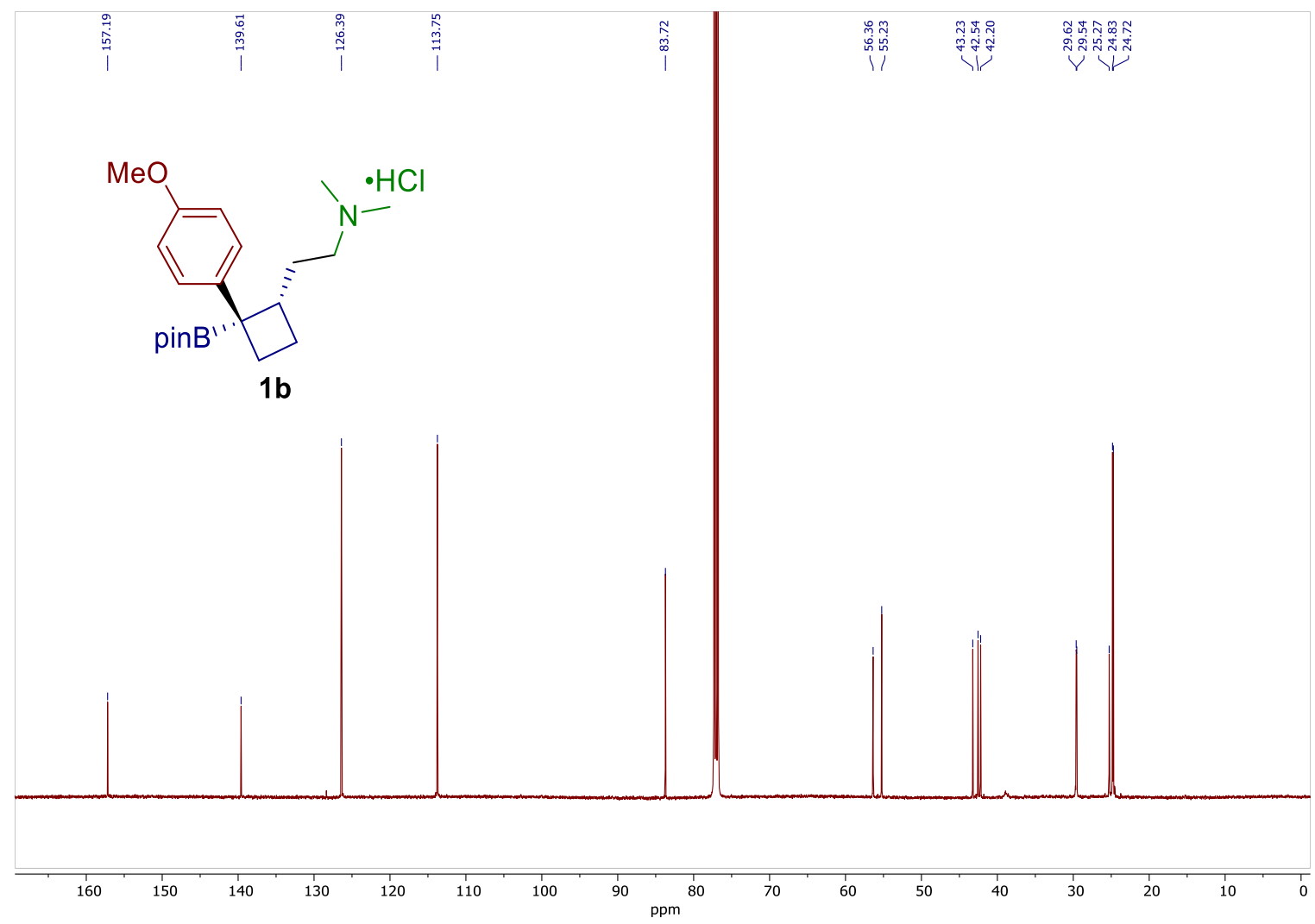


1H-NMR (400 MHz, $\left.\mathrm{CDCl}_{3}\right)$ of compound 1c see procedure

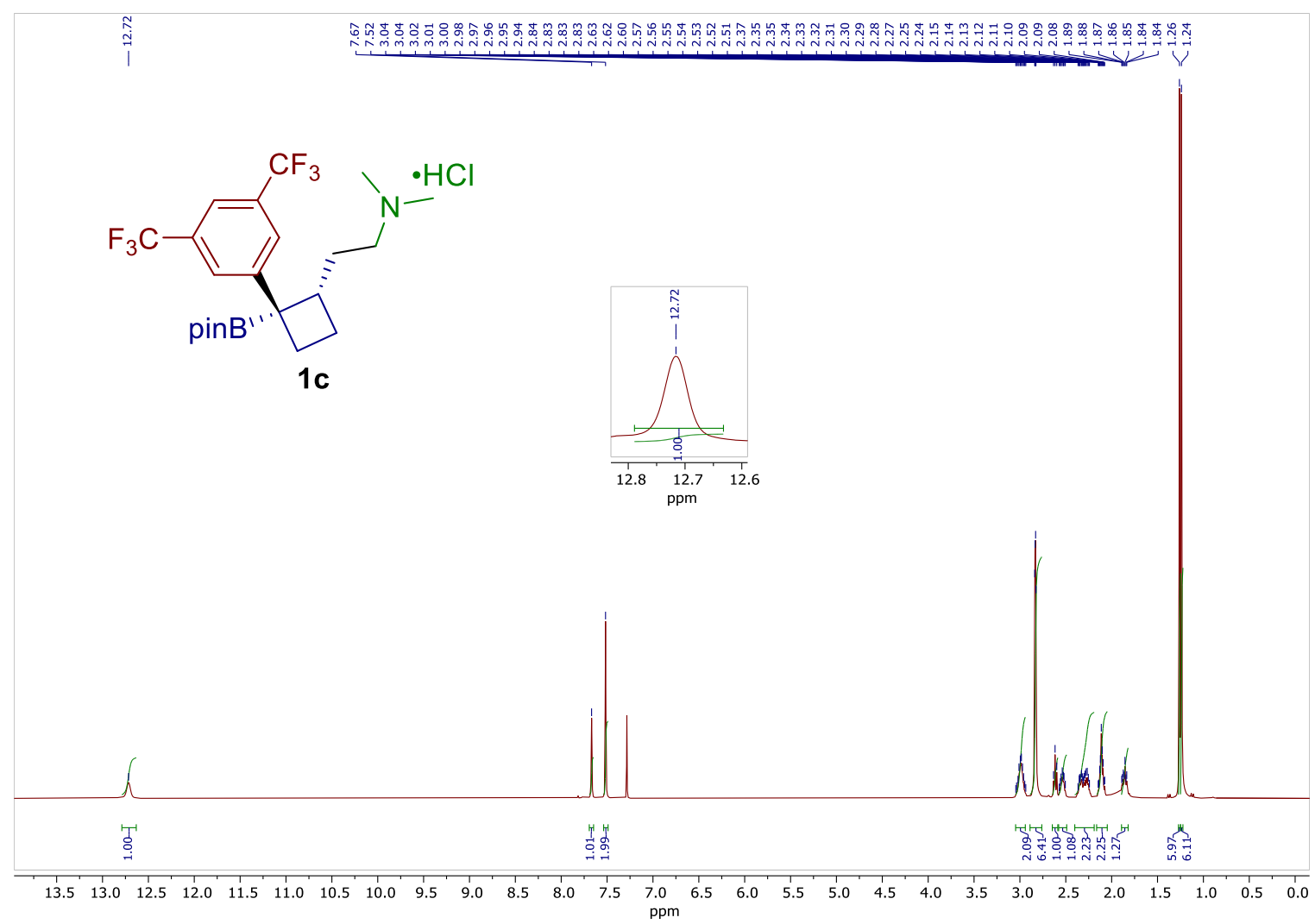

${ }^{13} \mathbf{C}-\mathbf{N M R}\left(100 \mathrm{MHz}, \mathrm{CDCl}_{3}\right)$ of compound 1c

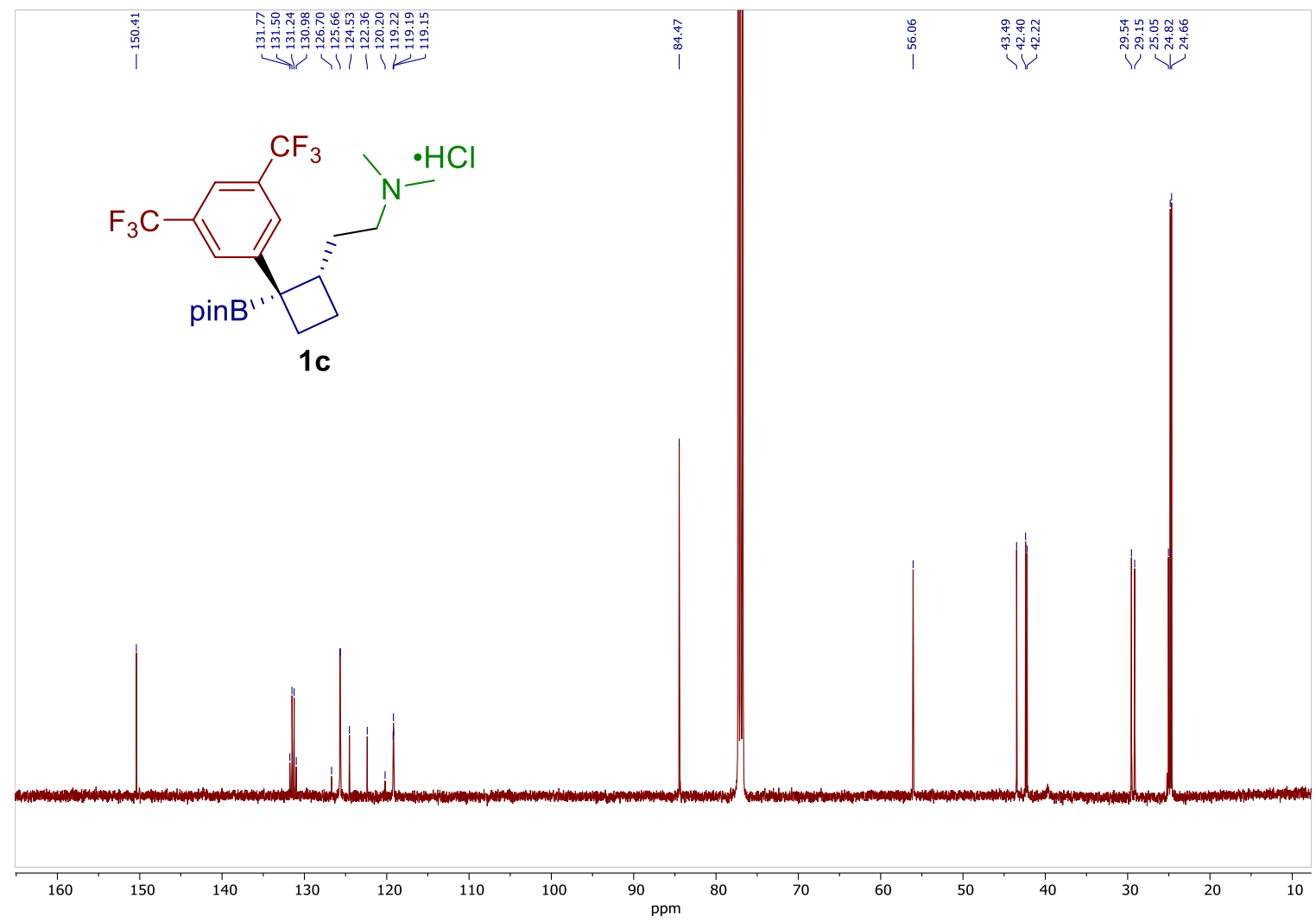


${ }^{19} \mathbf{F}-\mathbf{N M R}\left(376 \mathrm{MHz}, \mathrm{CDCl}_{3}\right)$ of compound 1c

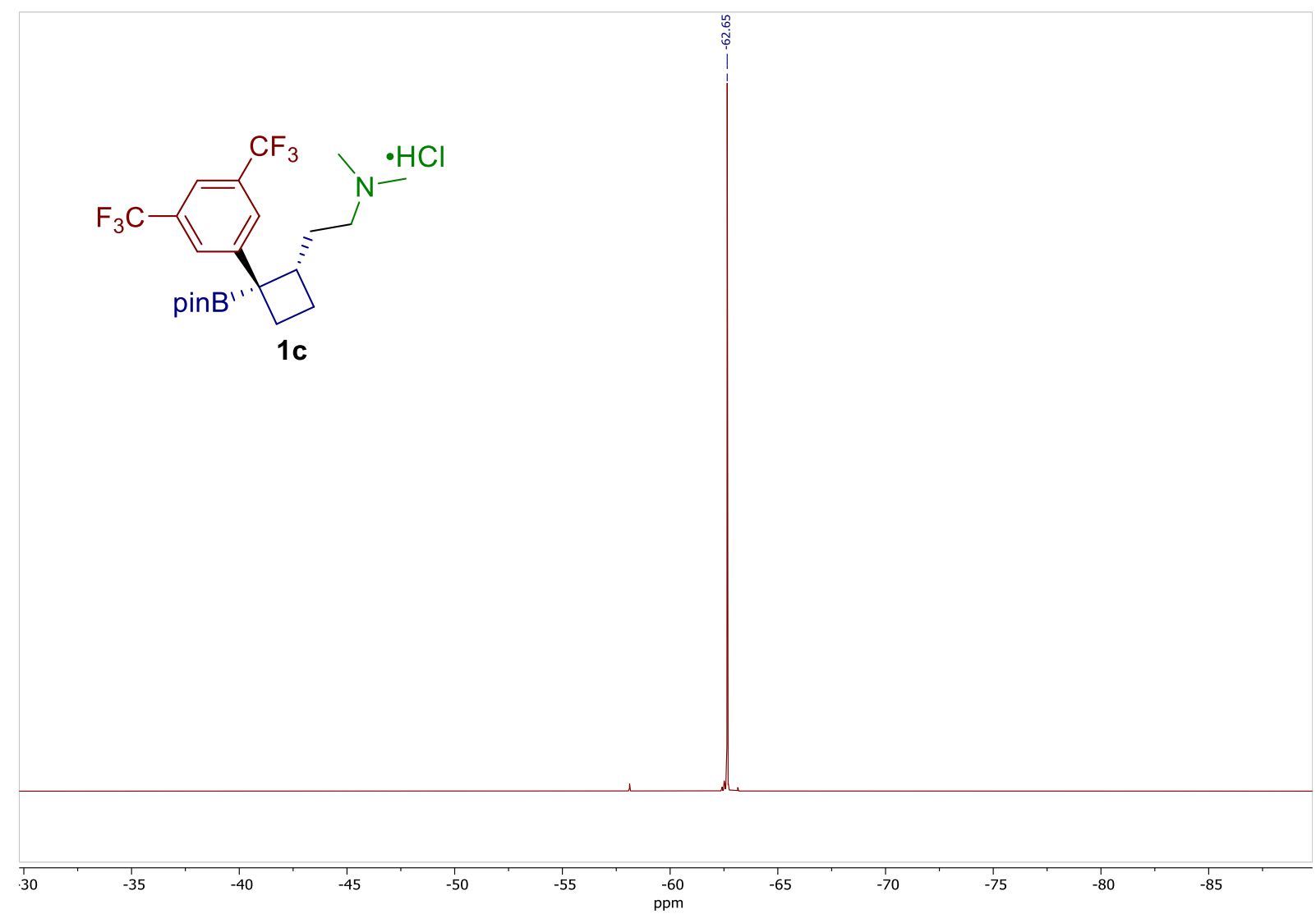


${ }^{1} \mathbf{H}-\mathbf{N M R}\left(400 \mathrm{MHz}, \mathrm{CDCl}_{3}\right)$ of compound $\mathbf{1 d}$ see procedure

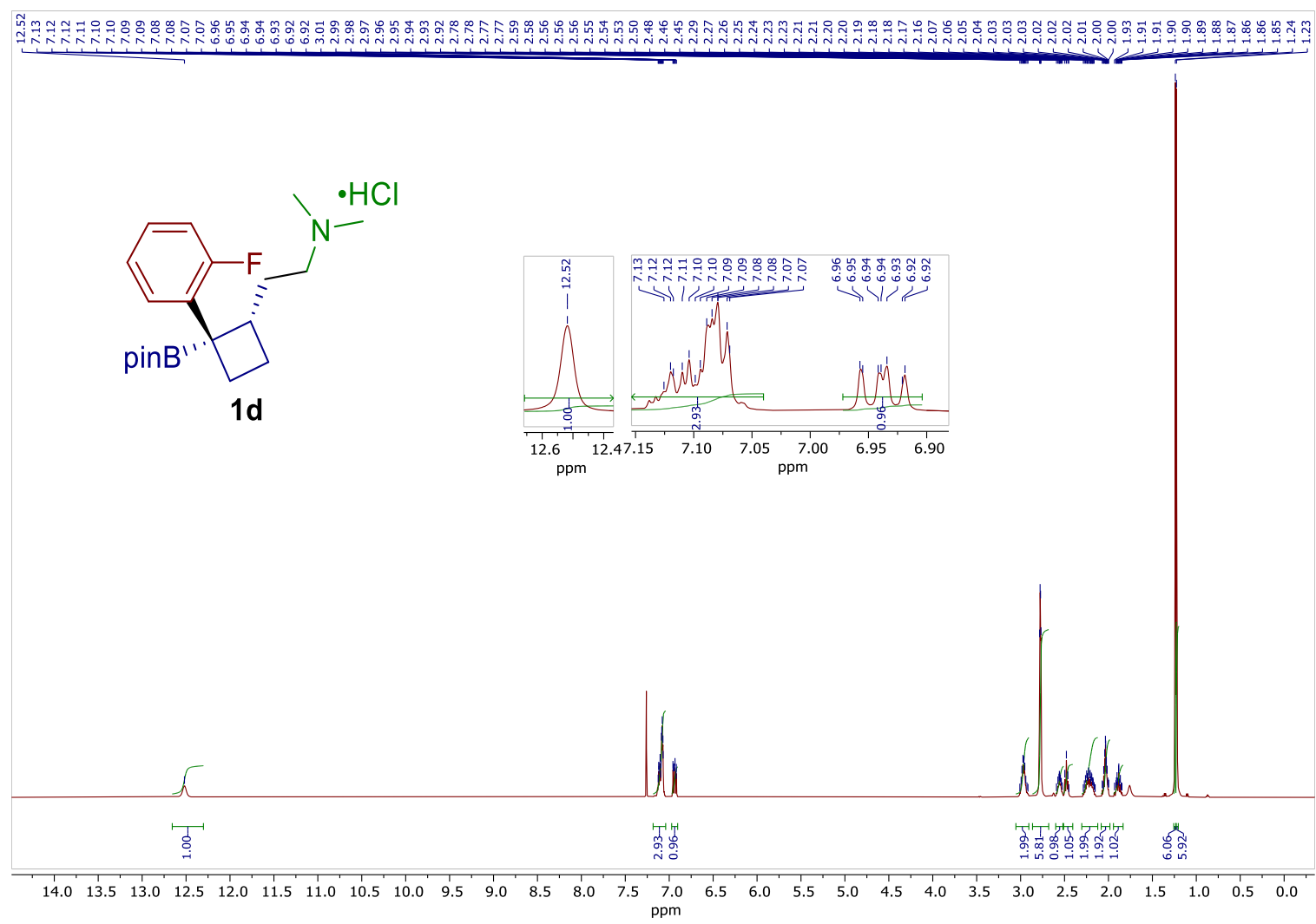

${ }^{13} \mathbf{C}$-NMR (100 MHz, $\left.\mathrm{CDCl}_{3}\right)$ of compound 1d

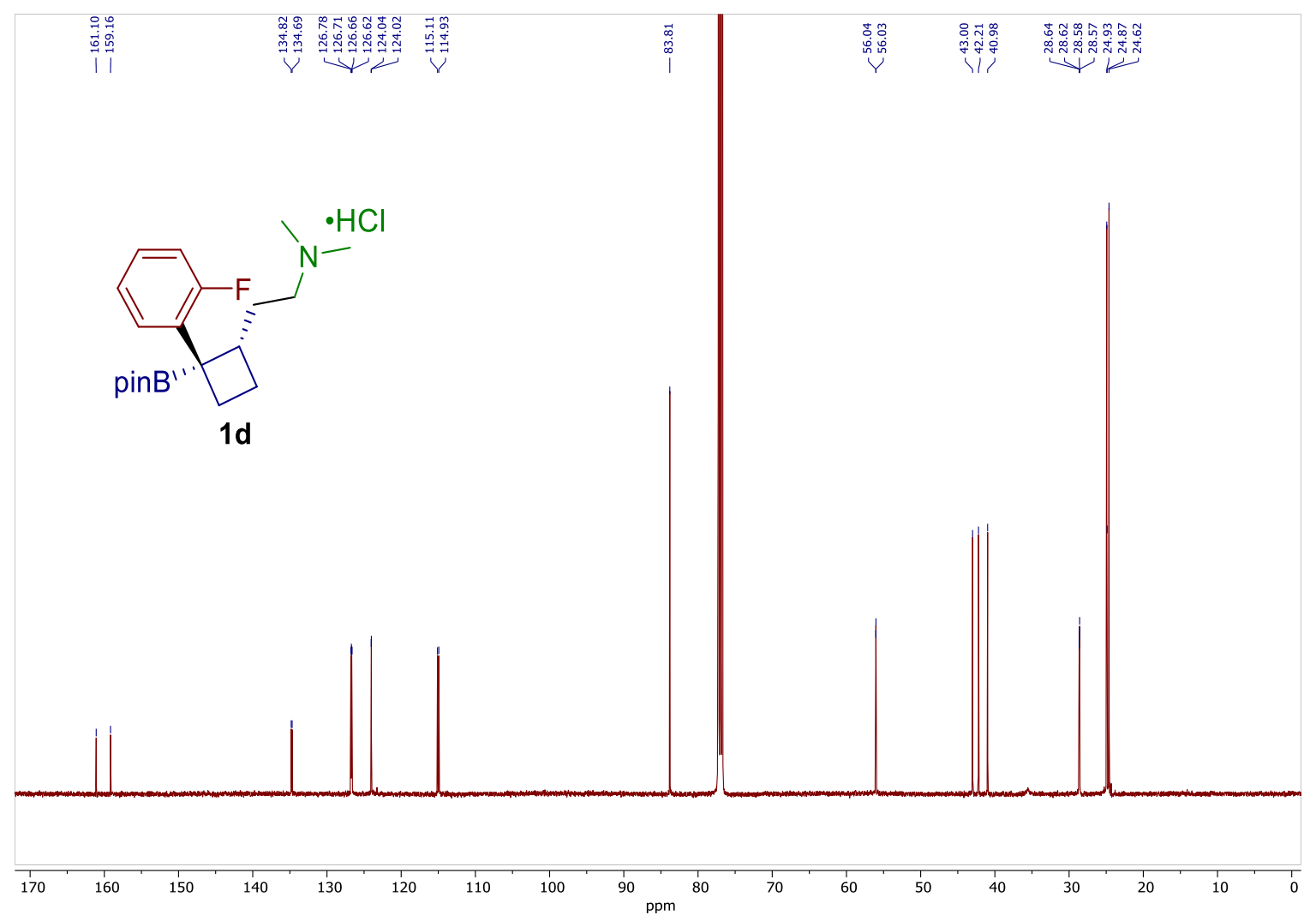


${ }^{19} \mathbf{F}$-NMR $\left(376 \mathrm{MHz}, \mathrm{CDCl}_{3}\right)$ of compound 1d

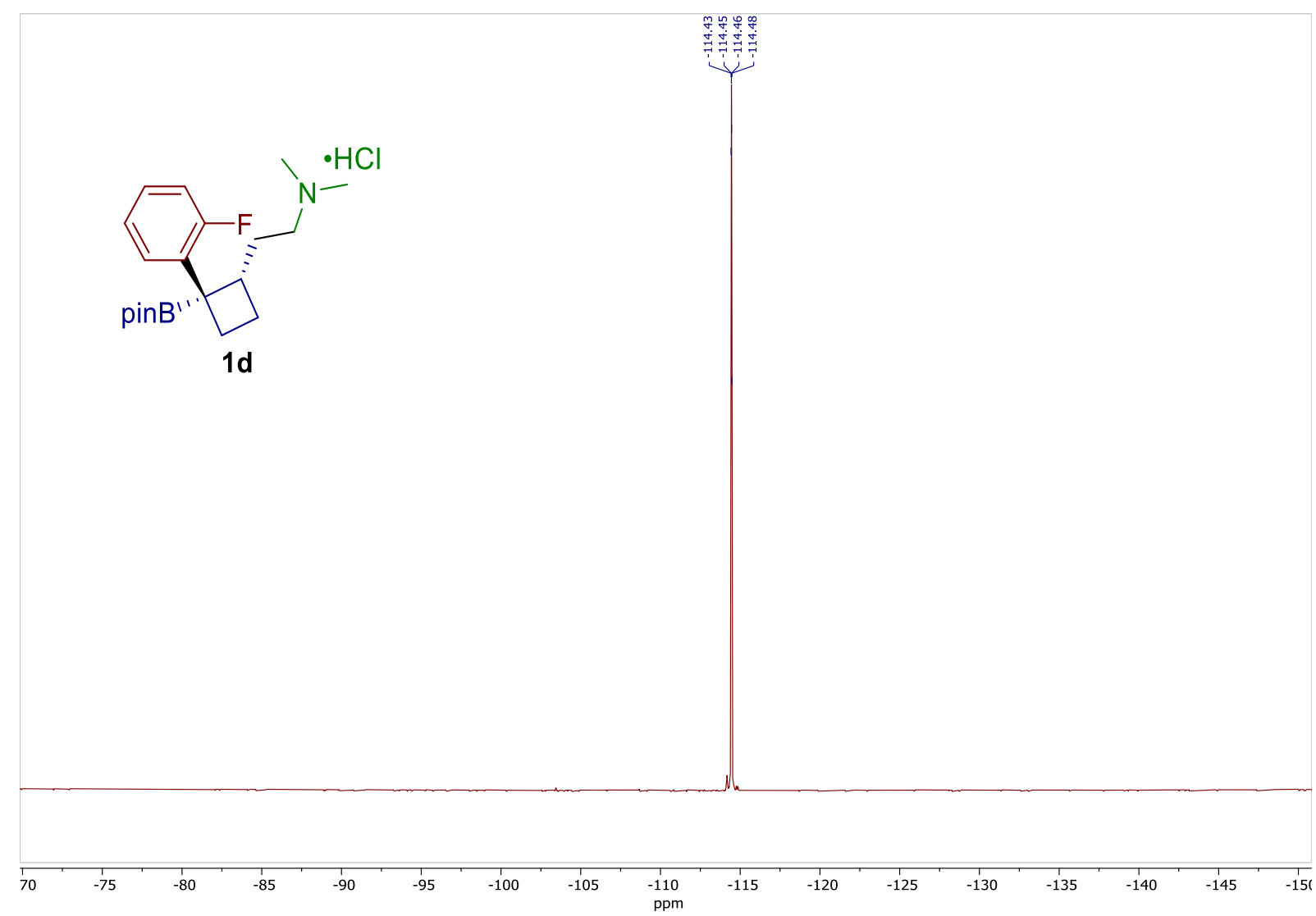


${ }^{1} \mathbf{H}-\mathbf{N M R}\left(400 \mathrm{MHz}, \mathrm{CDCl}_{3}\right)$ of compound $\mathbf{1 e}$ see procedure

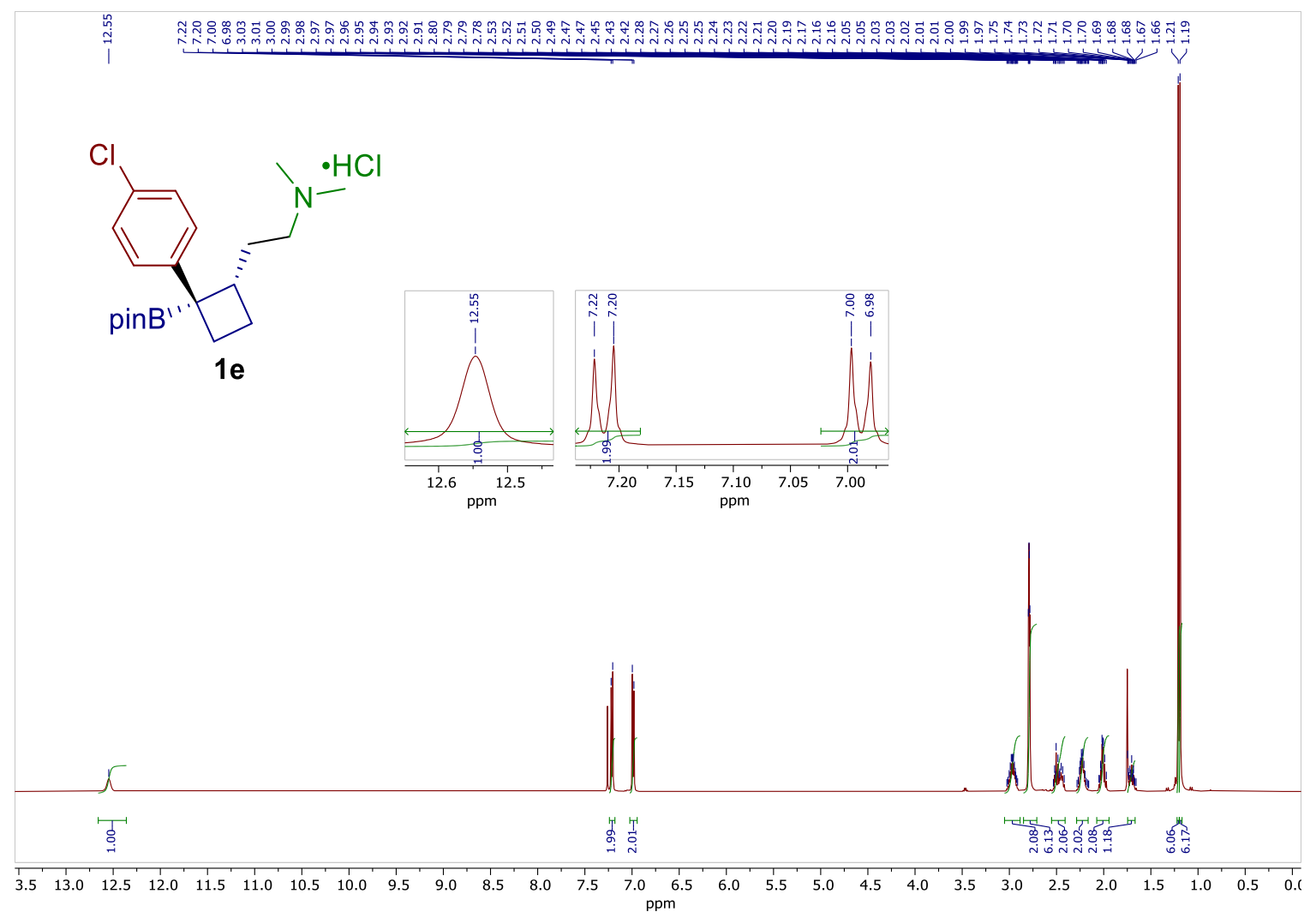

${ }^{13} \mathbf{C}-\mathbf{N M R}\left(100 \mathrm{MHz}, \mathrm{CDCl}_{3}\right)$ of compound $\mathbf{1 e}$

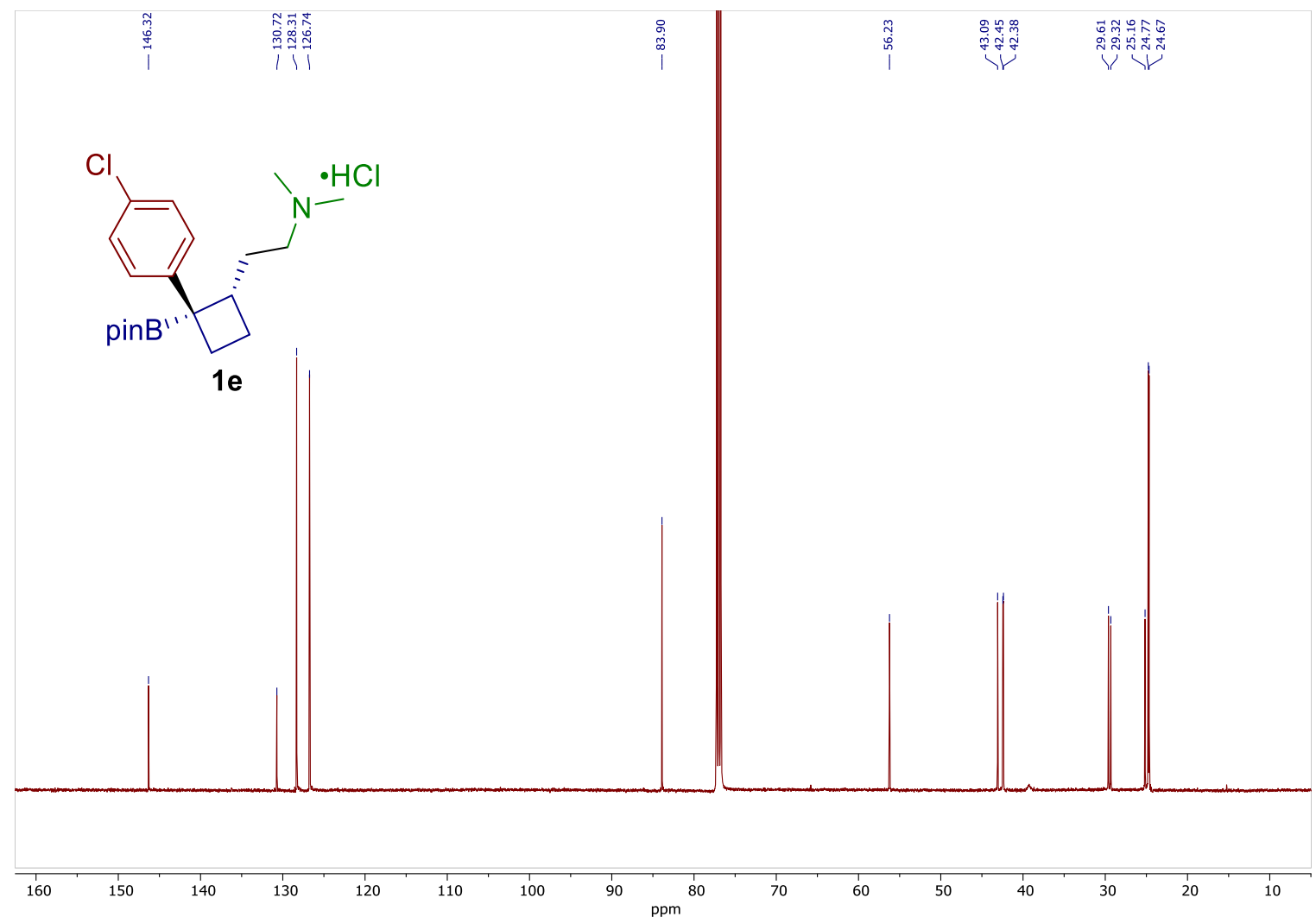


${ }^{\mathbf{1}} \mathbf{H}-\mathbf{N M R}\left(400 \mathrm{MHz}, \mathrm{CDCl}_{3}\right)$ of compound $\mathbf{1 f}$ see procedure

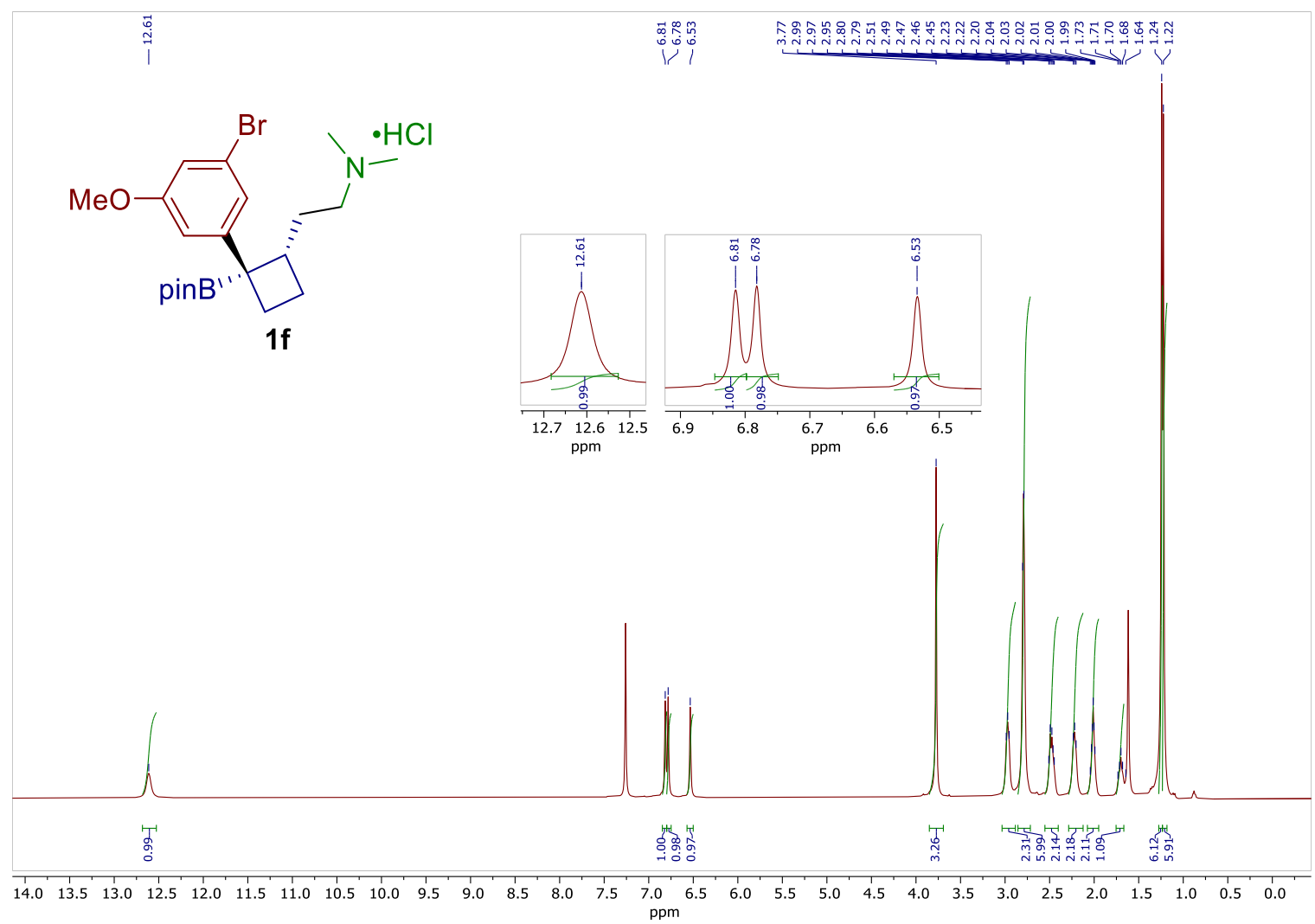

${ }^{13} \mathbf{C}$-NMR $\left(100 \mathrm{MHz}, \mathrm{CDCl}_{3}\right)$ of compound $\mathbf{1 f}$

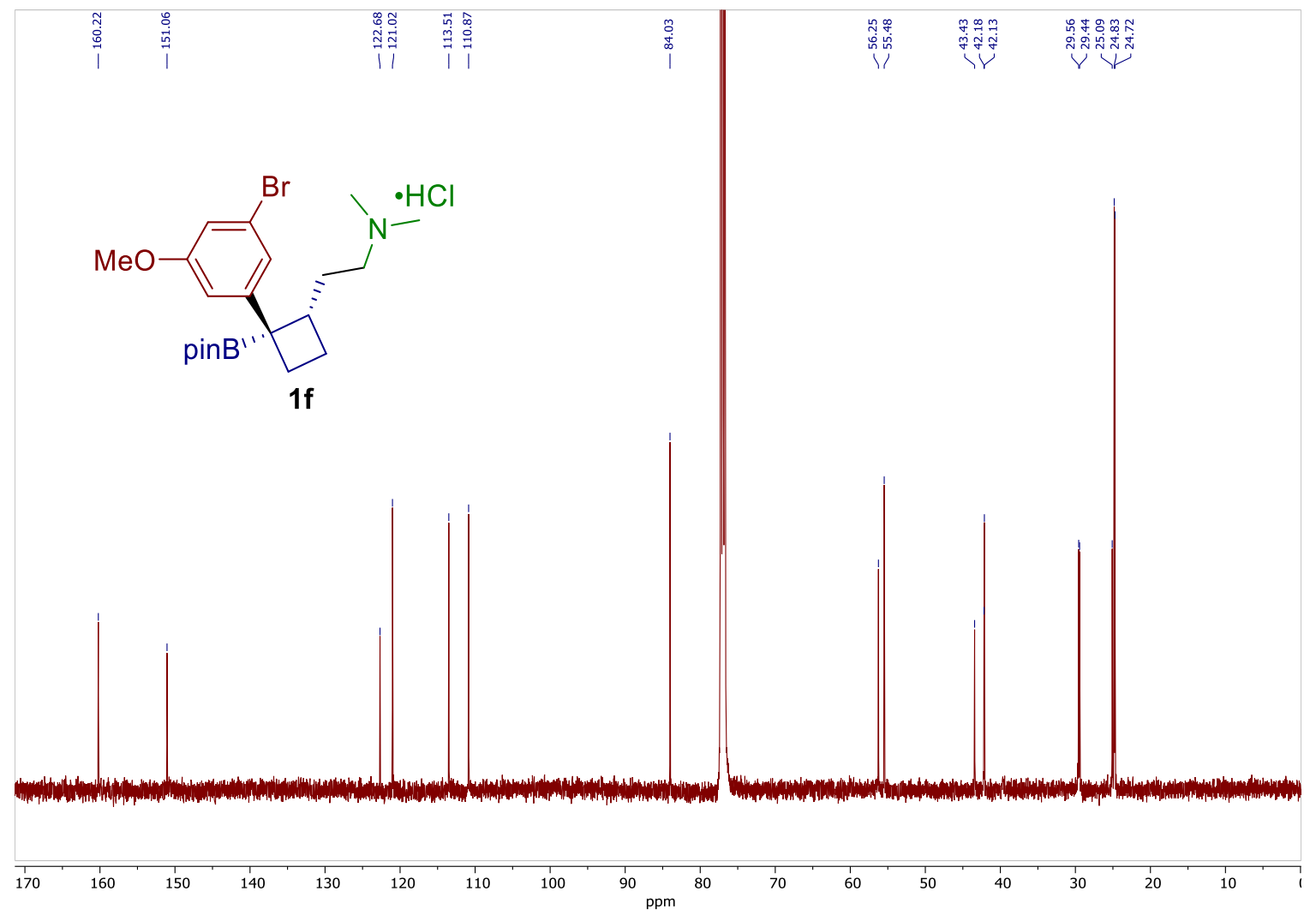


${ }^{\mathbf{1}} \mathbf{H}-\mathbf{N M R}\left(400 \mathrm{MHz}, \mathrm{CDCl}_{3}\right)$ of compound $\mathbf{1 g}$ see procedure

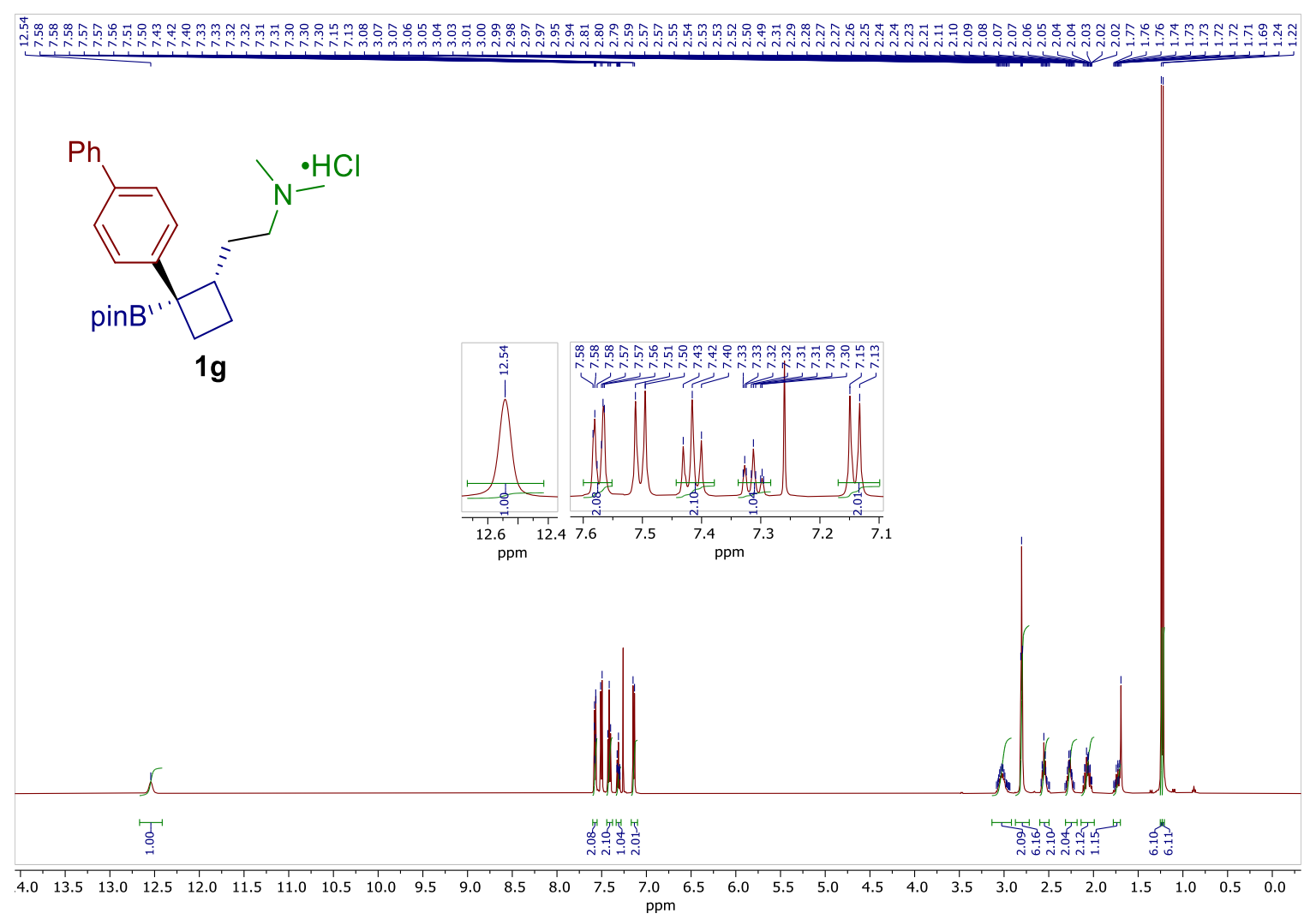

${ }^{13} \mathbf{C}-\mathbf{N M R}\left(100 \mathrm{MHz}, \mathrm{CDCl}_{3}\right)$ of compound $\mathbf{1 g}$

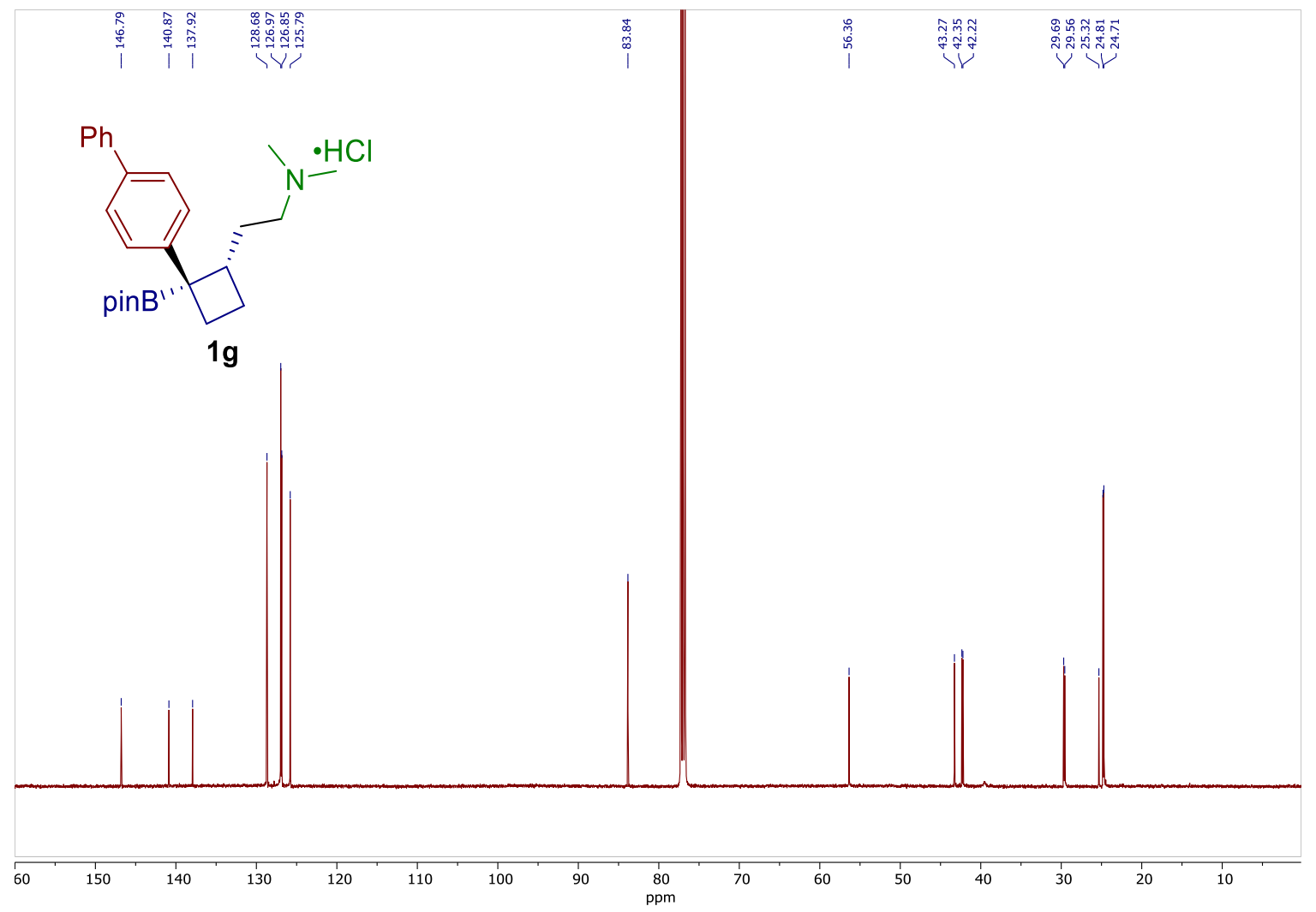


${ }^{\mathbf{1}} \mathbf{H}-\mathbf{N M R}\left(400 \mathrm{MHz}, \mathrm{CDCl}_{3}\right)$ of compound $\mathbf{1 h}$ see procedure

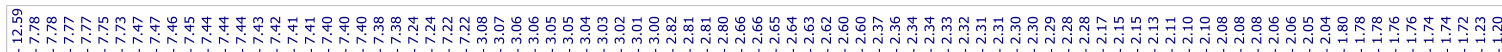

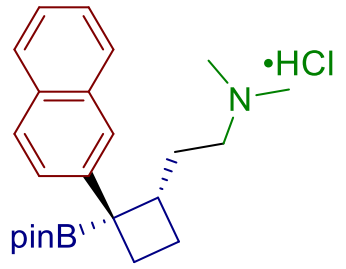

$1 \mathrm{~h}$

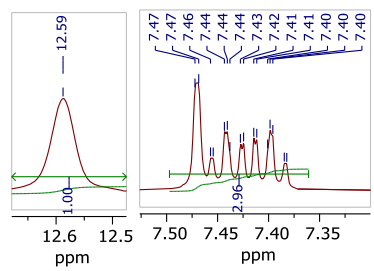

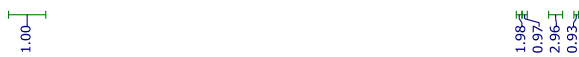

$\begin{array}{llllllllllllllllllllllllllllllllllll}4.5 & 14.0 & 13.5 & 13.0 & 12.5 & 12.0 & 11.5 & 11.0 & 10.5 & 10.0 & 9.5 & 9.0 & 8.5 & 8.0 & 7.5 & 7.0 & 6.5 & 6.0 & 5.5 & 5.0 & 4.5 & 4.0 & 3.5 & 3.0 & 2.5 & 2.0 & 1.5 & 1.0 & 0.5 & 0.0\end{array}$

${ }^{13} \mathbf{C}-\mathrm{NMR}\left(100 \mathrm{MHz}, \mathrm{CDCl}_{3}\right)$ of compound $\mathbf{1 h}$

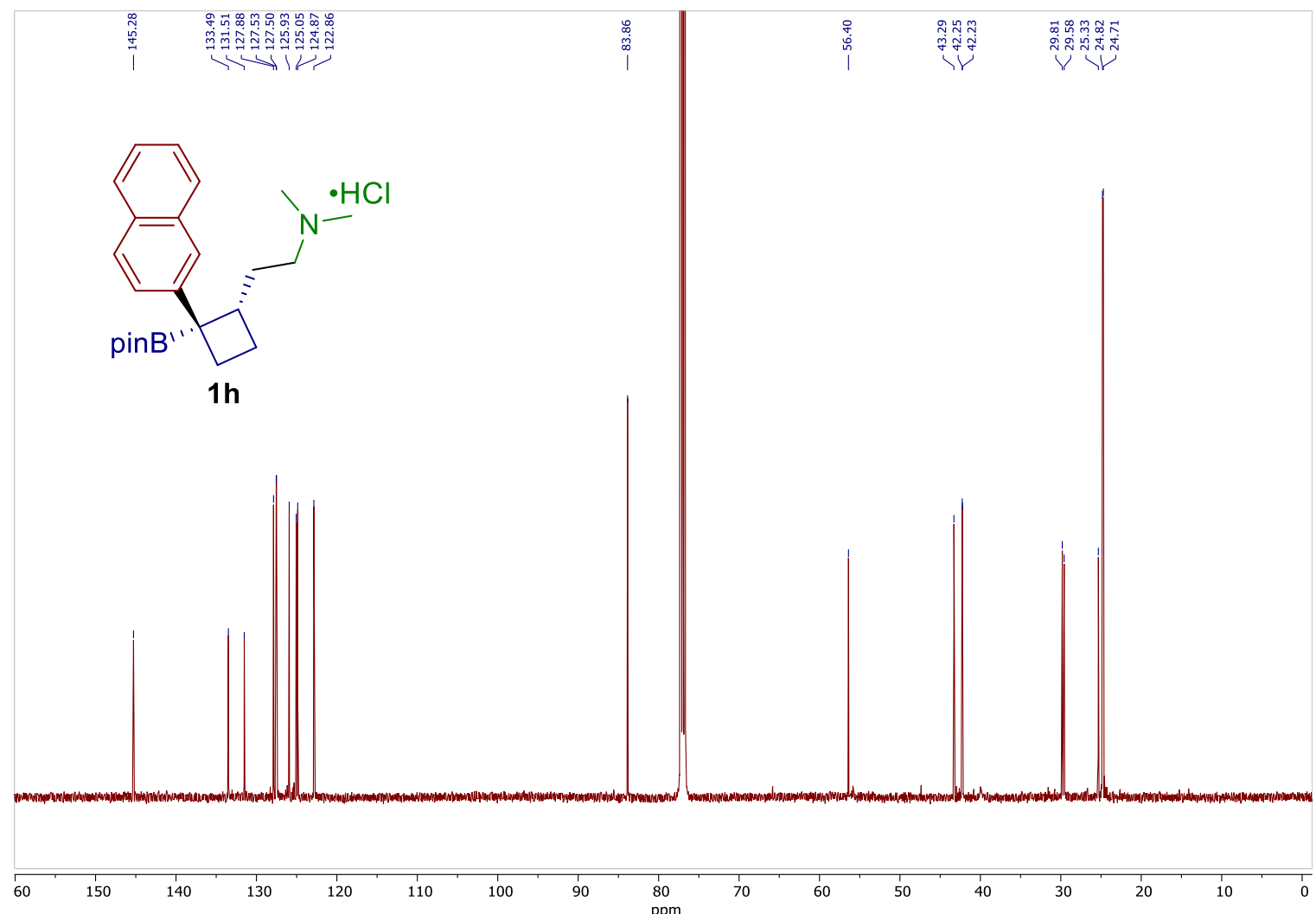


${ }^{1} \mathbf{H}-\mathbf{N M R}\left(400 \mathrm{MHz}, \mathrm{CDCl}_{3}\right)$ of compound $\mathbf{1 i}$ see procedure

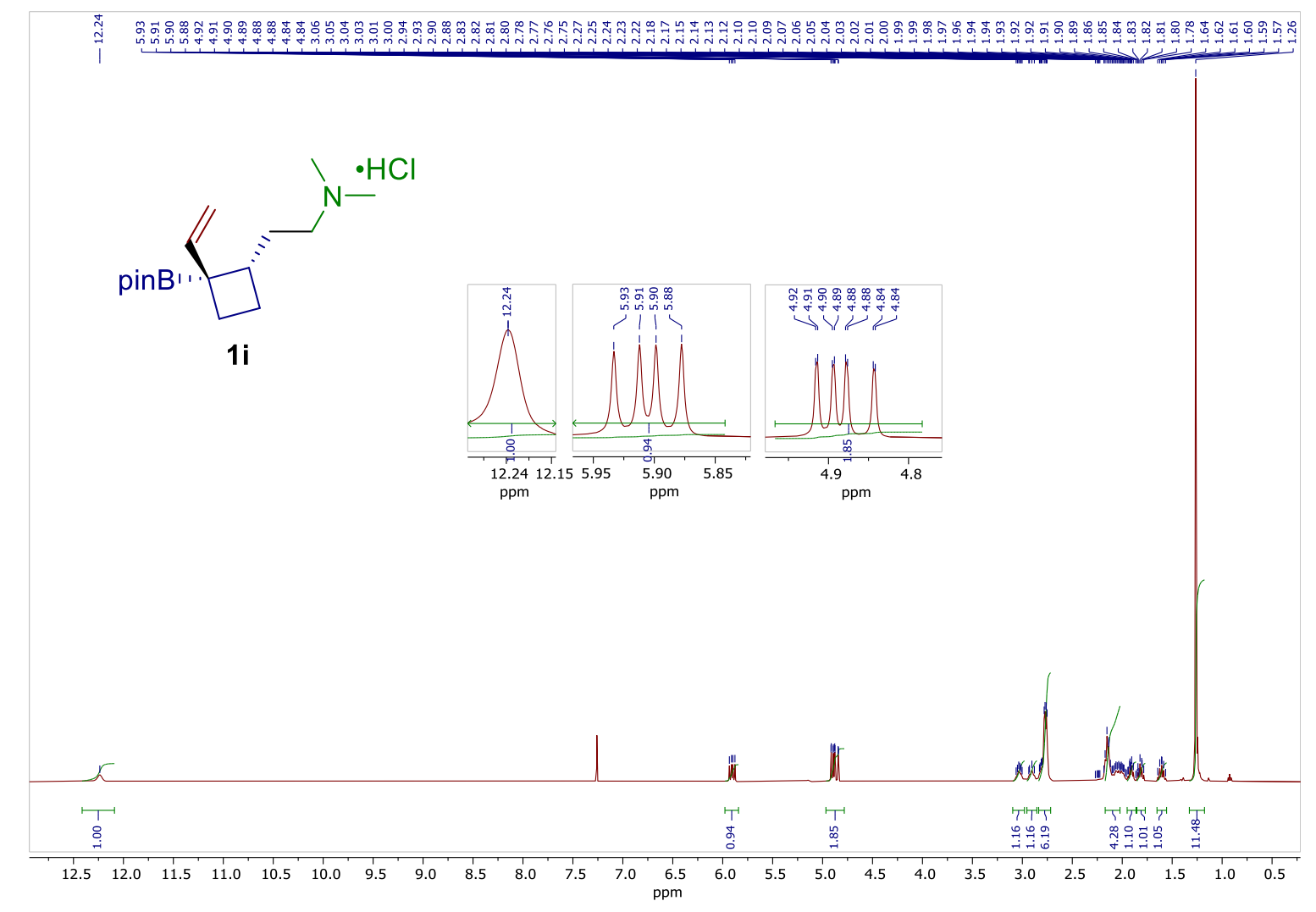

${ }^{13} \mathbf{C}-\mathbf{N M R}\left(100 \mathrm{MHz}, \mathrm{CDCl}_{3}\right)$ of compound $\mathbf{1 i}$

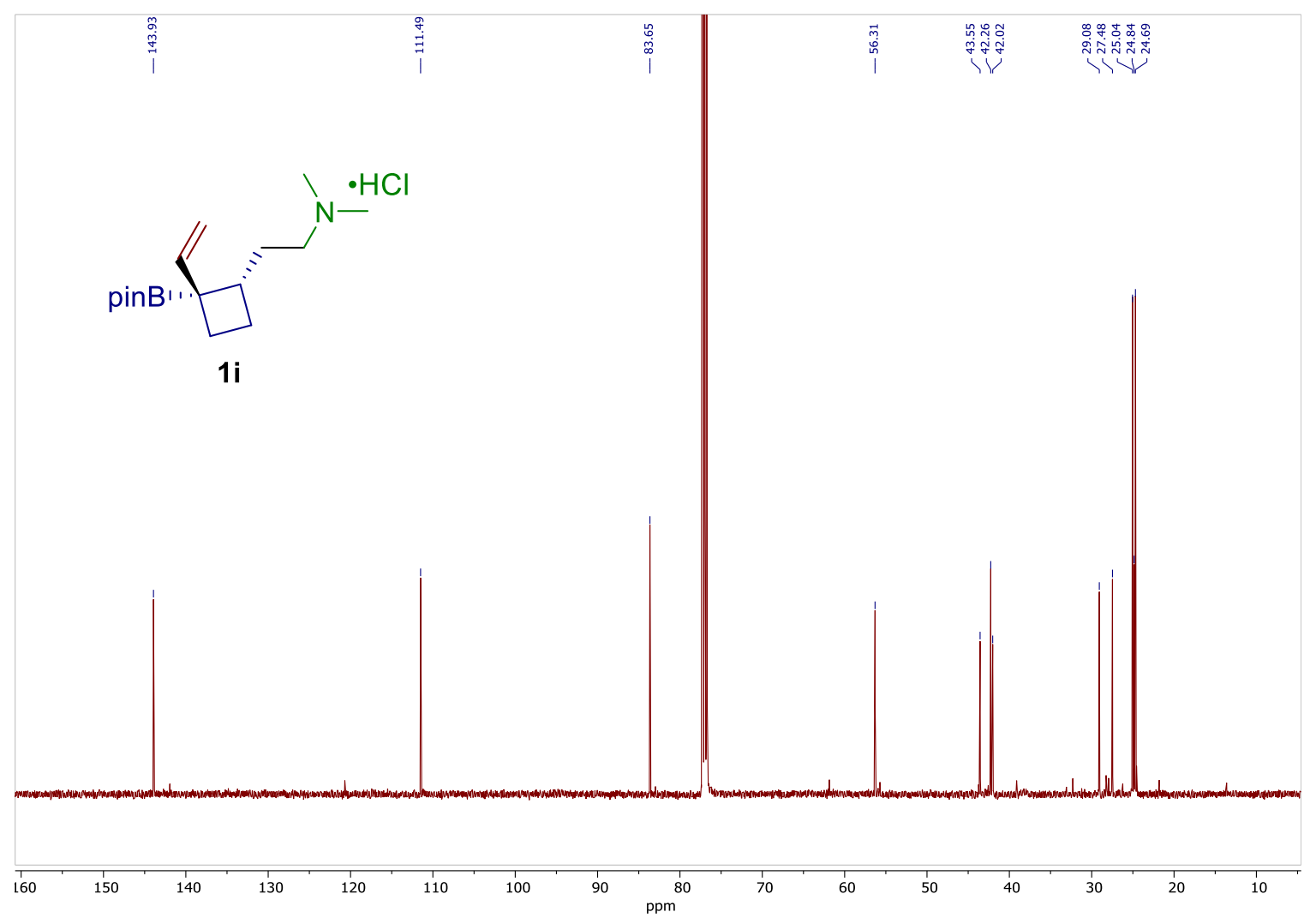


${ }^{\mathbf{1}} \mathbf{H}-\mathbf{N M R}\left(400 \mathrm{MHz}, \mathrm{CDCl}_{3}\right)$ of compound $\mathbf{1} \mathbf{j}$ see procedure<smiles>C/C=C/[C@@]1([PbH])CC[C@@H]1CCN(C)C</smiles>

1j
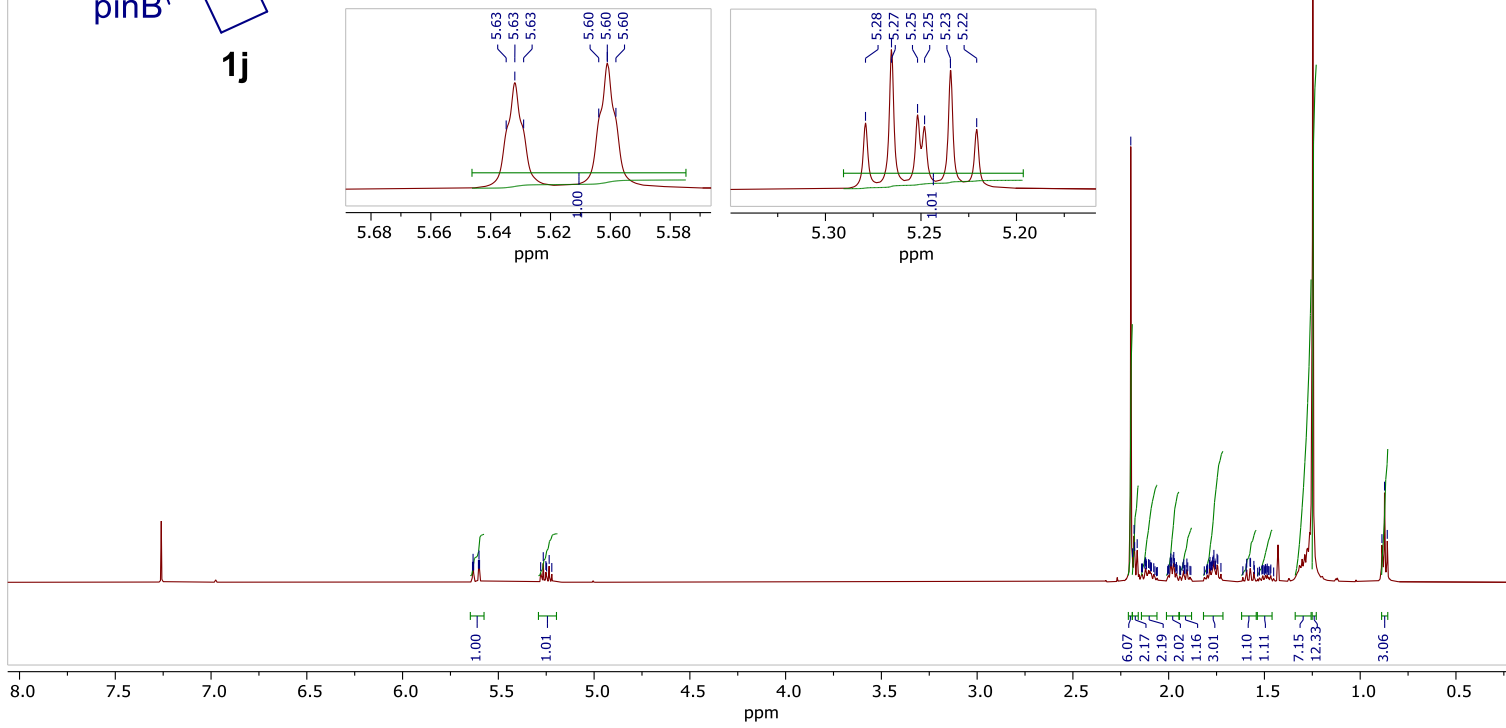

${ }^{13} \mathbf{C}$-NMR $\left(100 \mathrm{MHz}, \mathrm{CDCl}_{3}\right)$ of compound $\mathbf{1 j}$

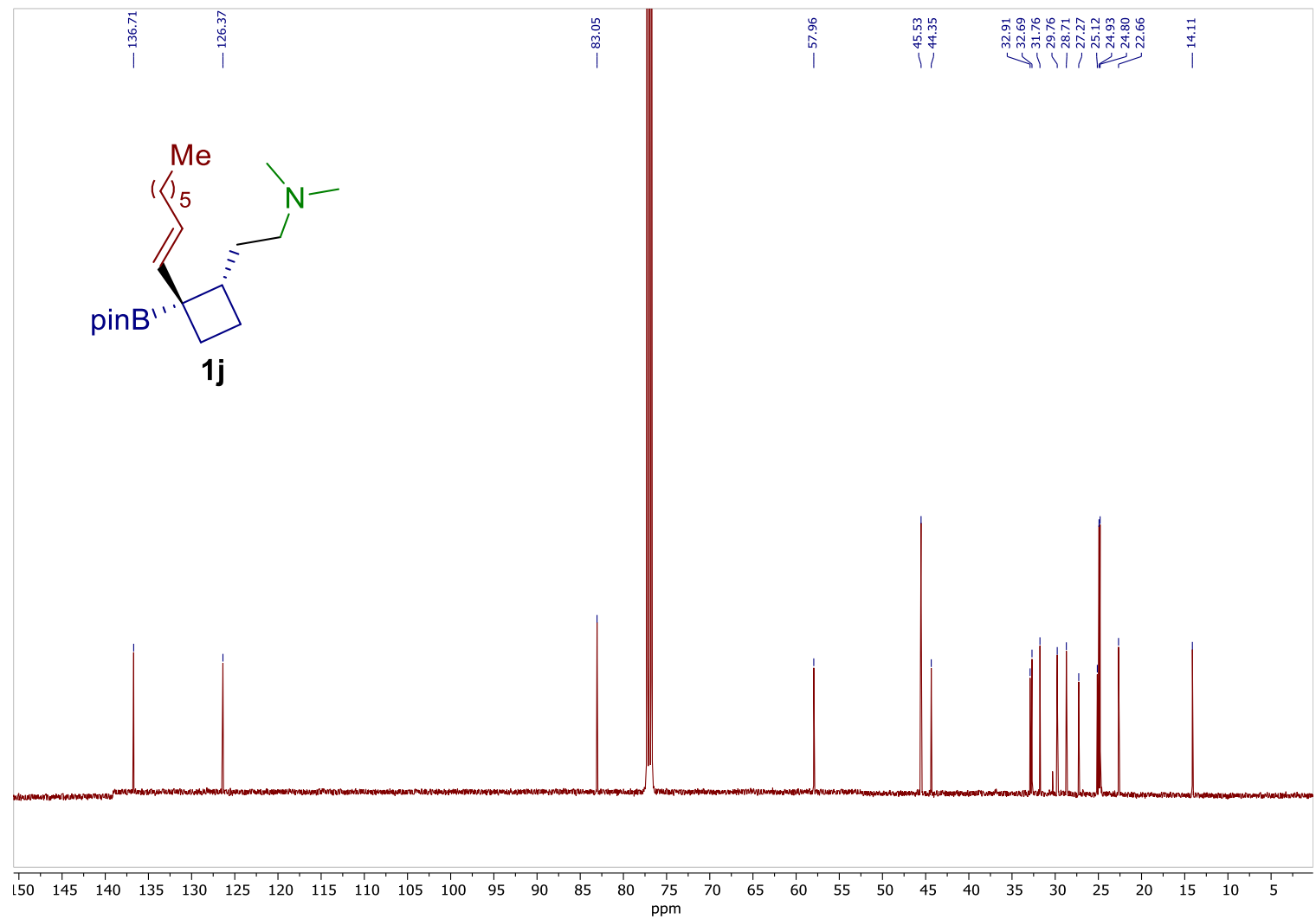


${ }^{\mathbf{1}} \mathbf{H}-\mathbf{N M R}\left(400 \mathrm{MHz}, \mathrm{CDCl}_{3}\right)$ of compound $\mathbf{1 k}$ see procedure

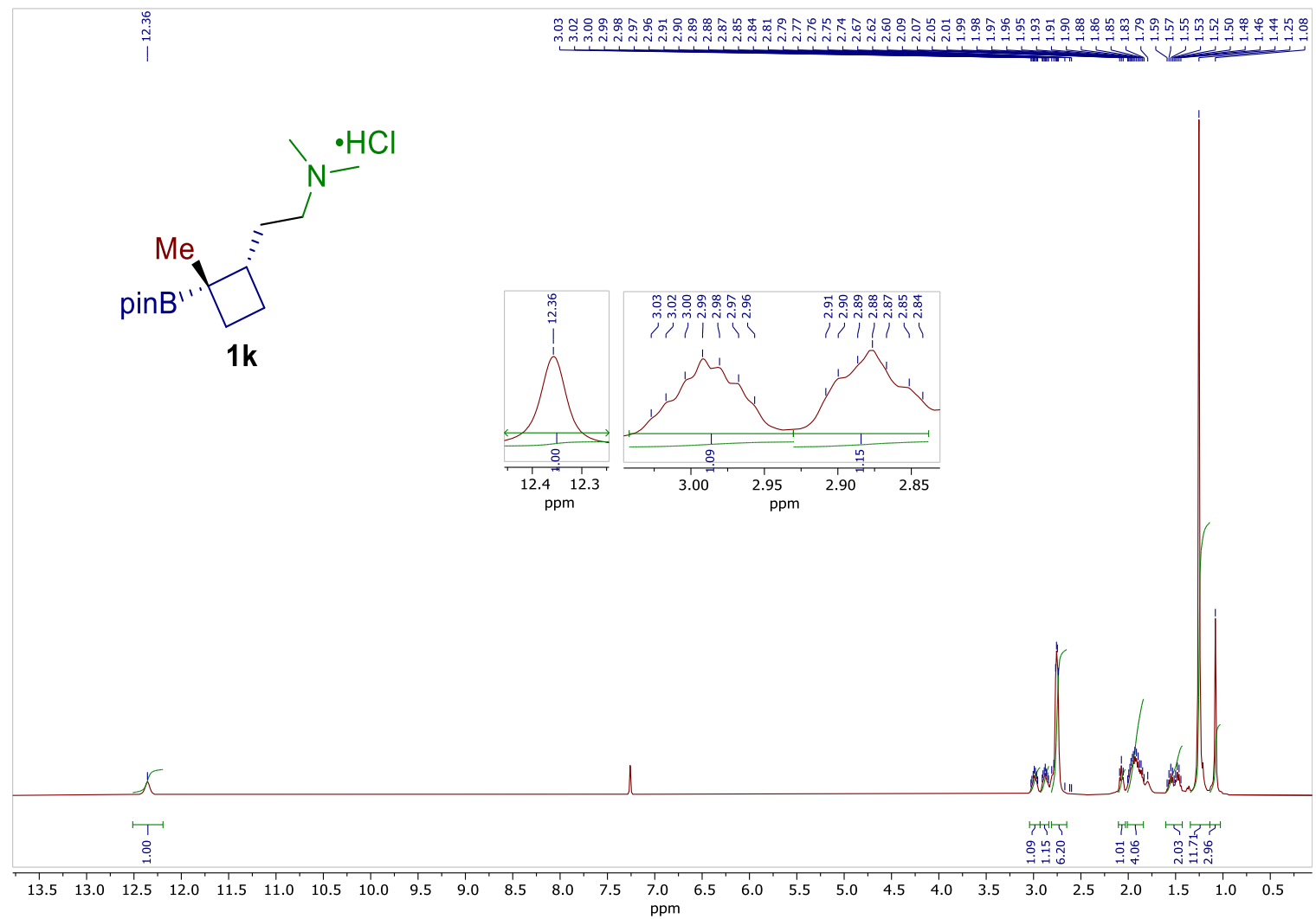

${ }^{13} \mathbf{C}$-NMR (100 MHz, $\left.\mathrm{CDCl}_{3}\right)$ of compound $\mathbf{1 k}$

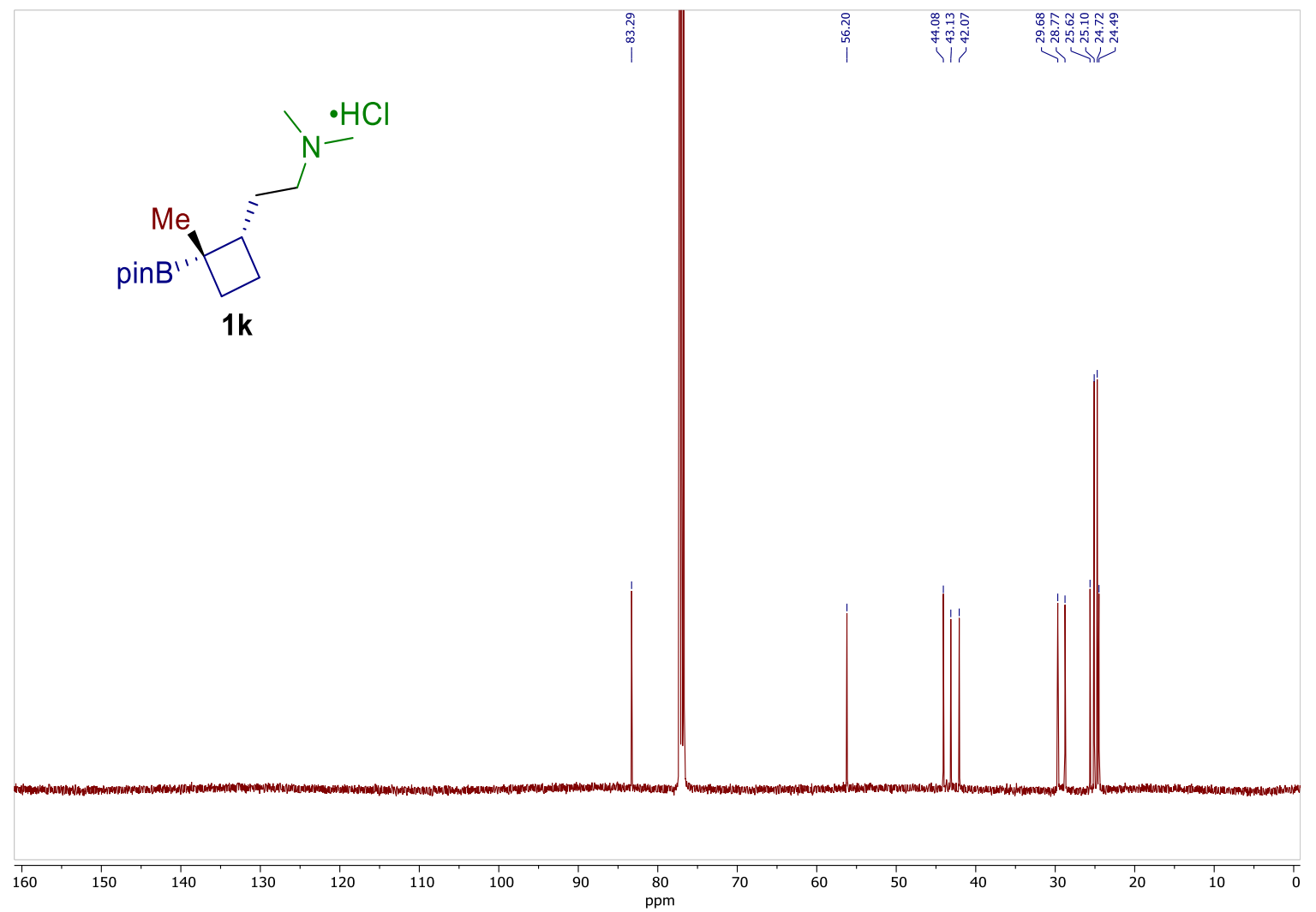


${ }^{1} \mathbf{H}-\mathbf{N M R}\left(400 \mathrm{MHz}, \mathrm{CDCl}_{3}\right)$ of compound $\mathbf{1 1}$ see procedure

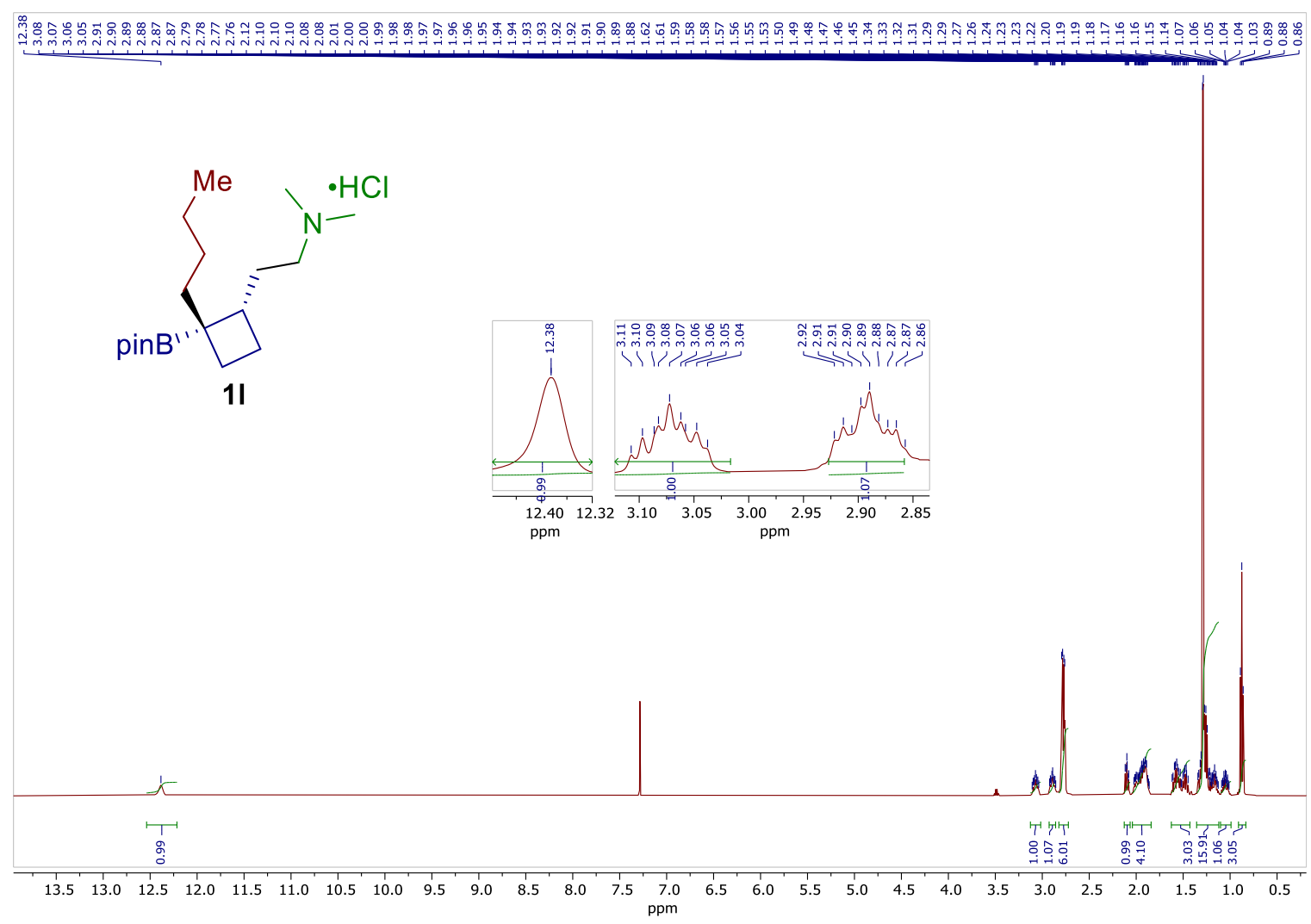

${ }^{13} \mathbf{C}-\mathbf{N M R}\left(100 \mathrm{MHz}, \mathrm{CDCl}_{3}\right)$ of compound $\mathbf{1 l}$

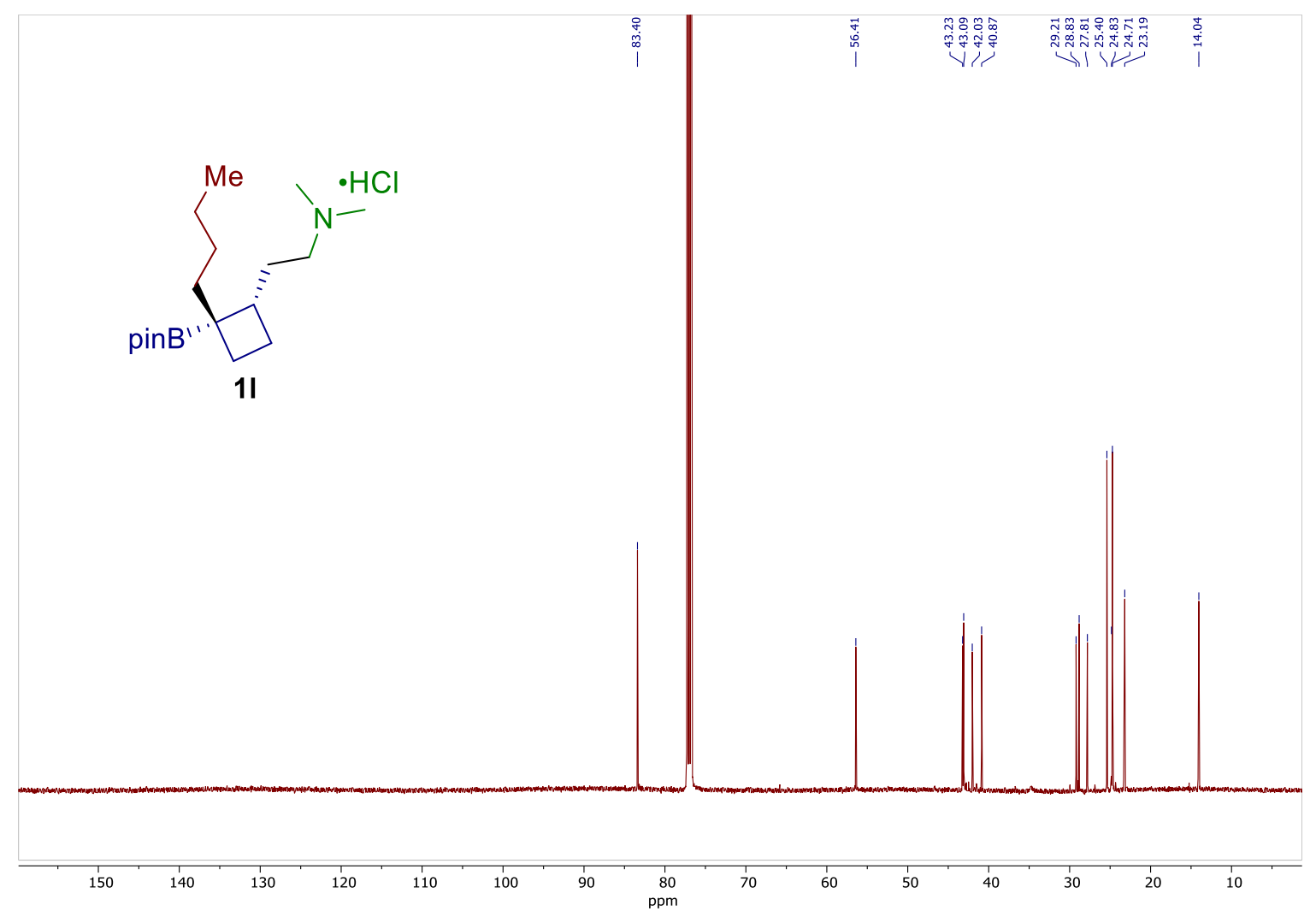


${ }^{\mathbf{1}} \mathbf{H}$-NMR $\left(400 \mathrm{MHz}, \mathrm{CDCl}_{3}\right)$ of compound $\mathbf{1 m}$ see procedure

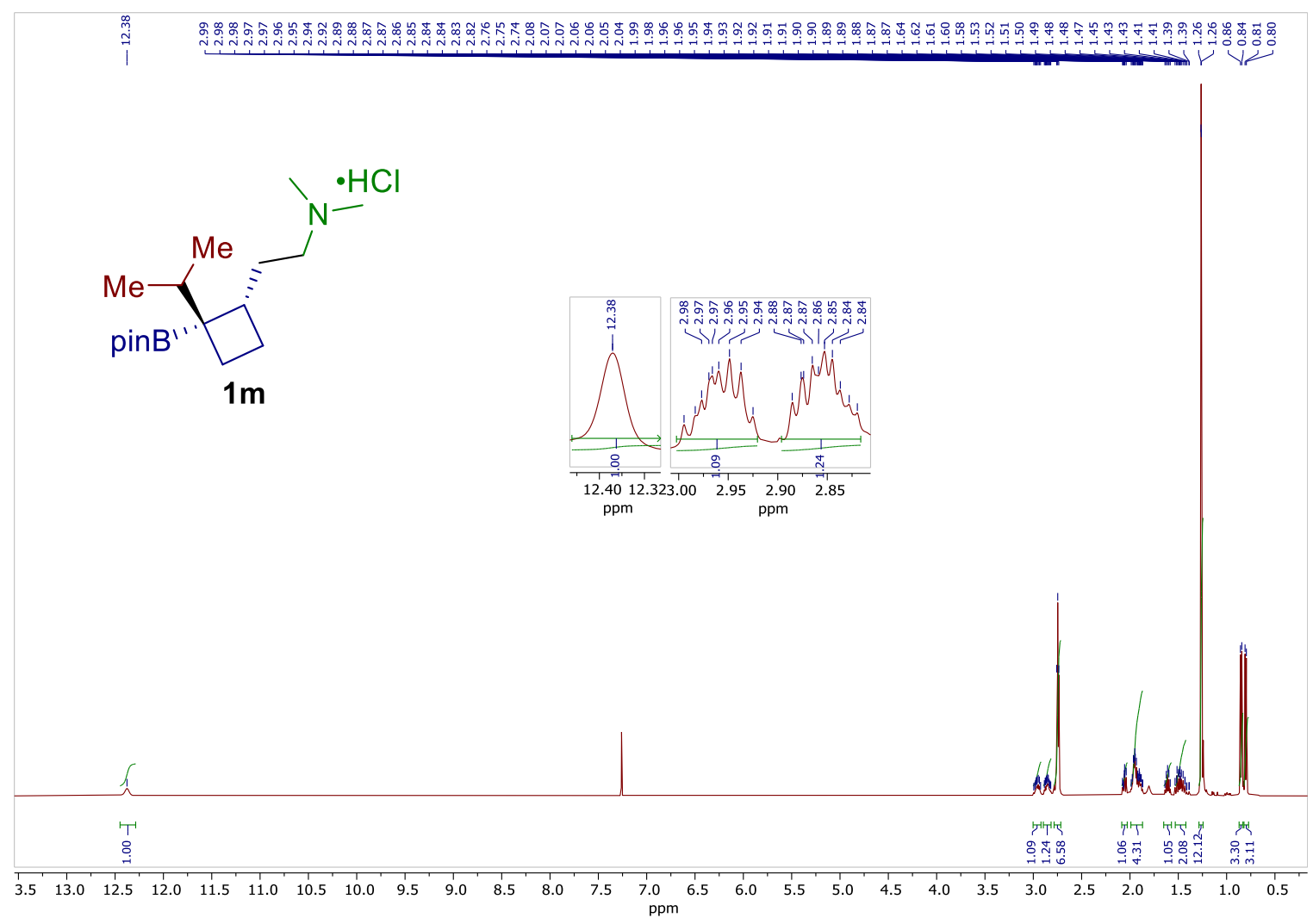

${ }^{13} \mathbf{C}$-NMR (100 MHz, $\left.\mathrm{CDCl}_{3}\right)$ of compound $\mathbf{1 m}$

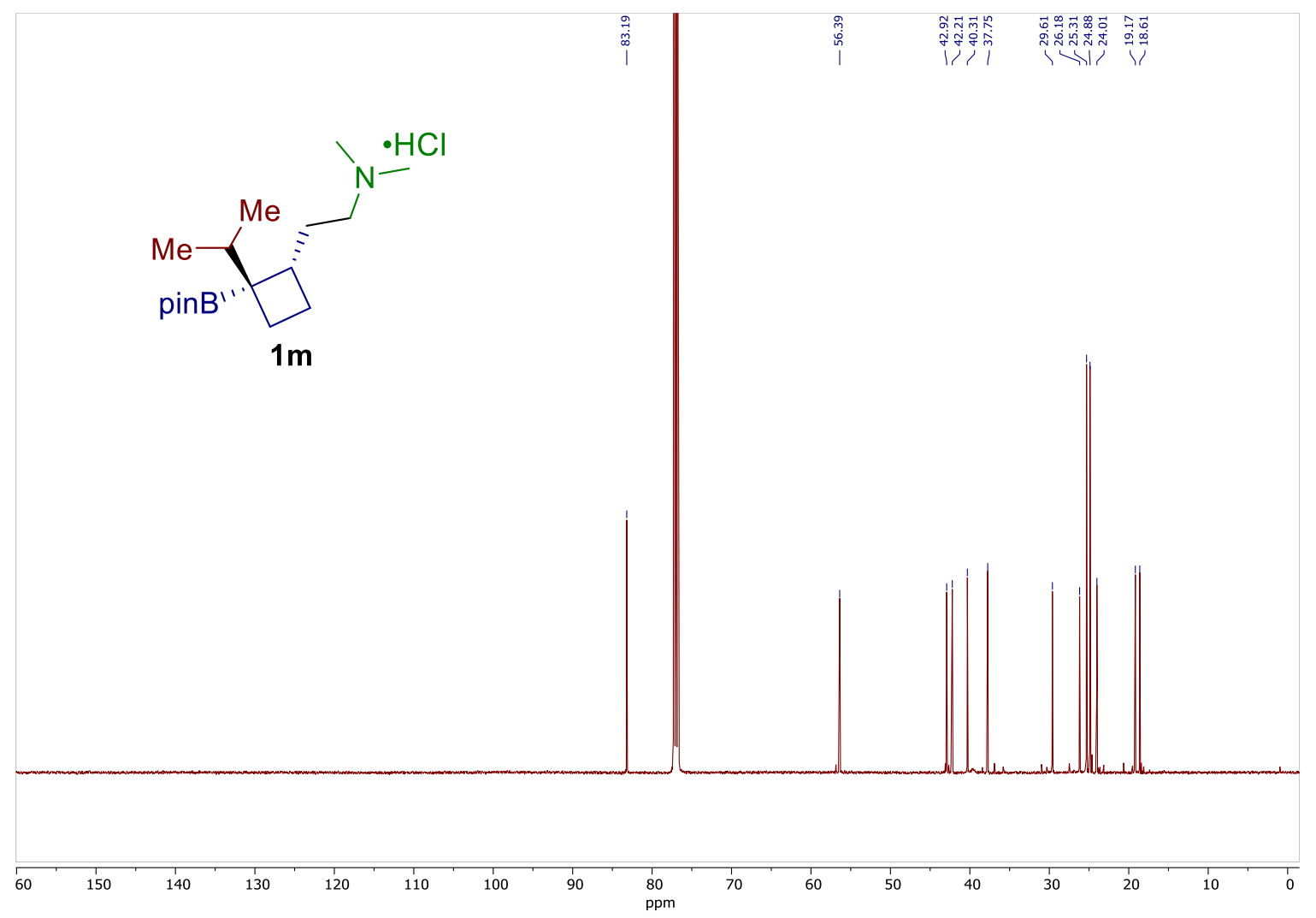


${ }^{\mathbf{1}} \mathbf{H}-\mathbf{N M R}\left(400 \mathrm{MHz}, \mathrm{CDCl}_{3}\right)$ of compound $\mathbf{1 n}$ see procedure

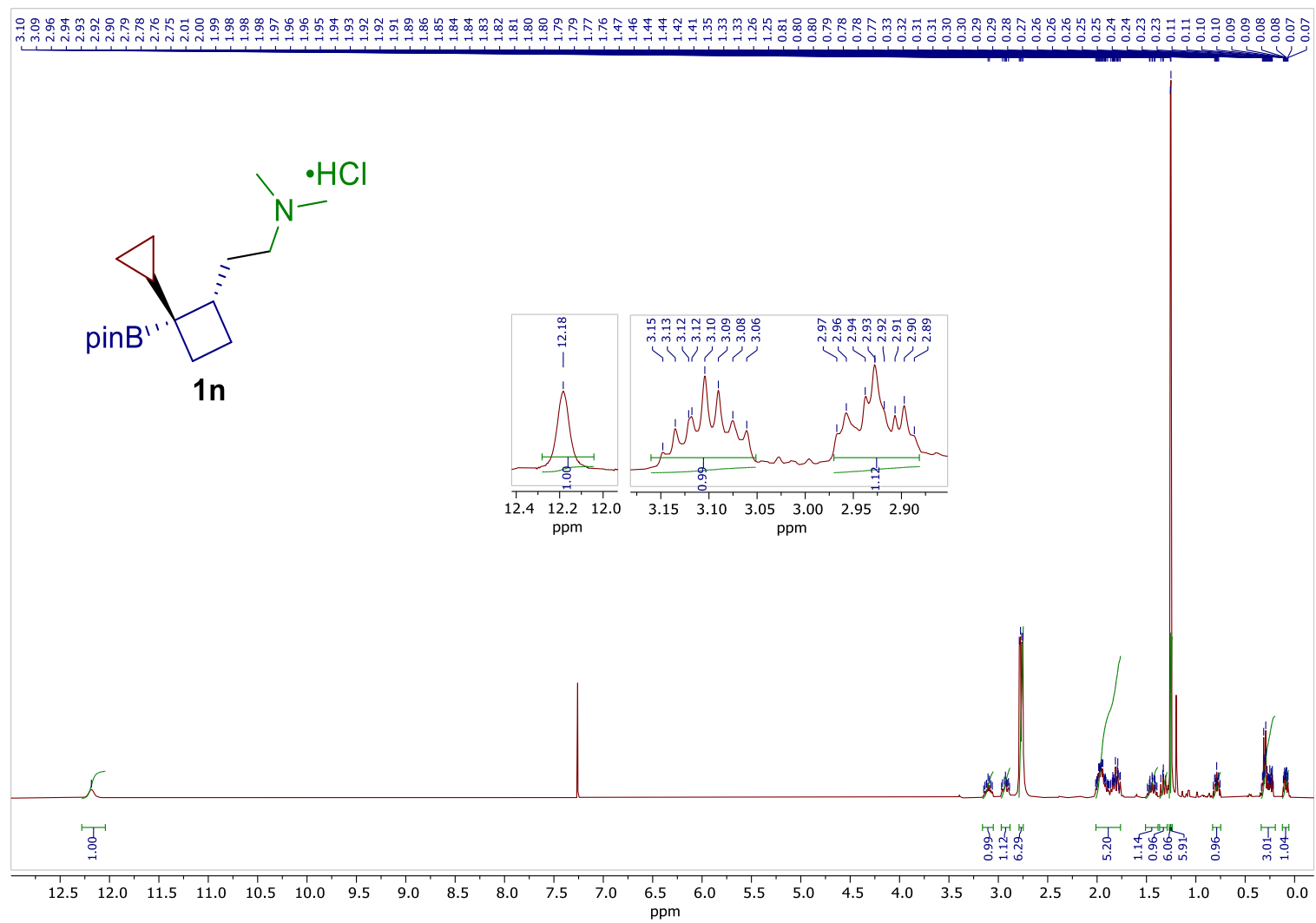

${ }^{13} \mathbf{C}$-NMR $\left(100 \mathrm{MHz}, \mathrm{CDCl}_{3}\right)$ of compound $\mathbf{1 n}$

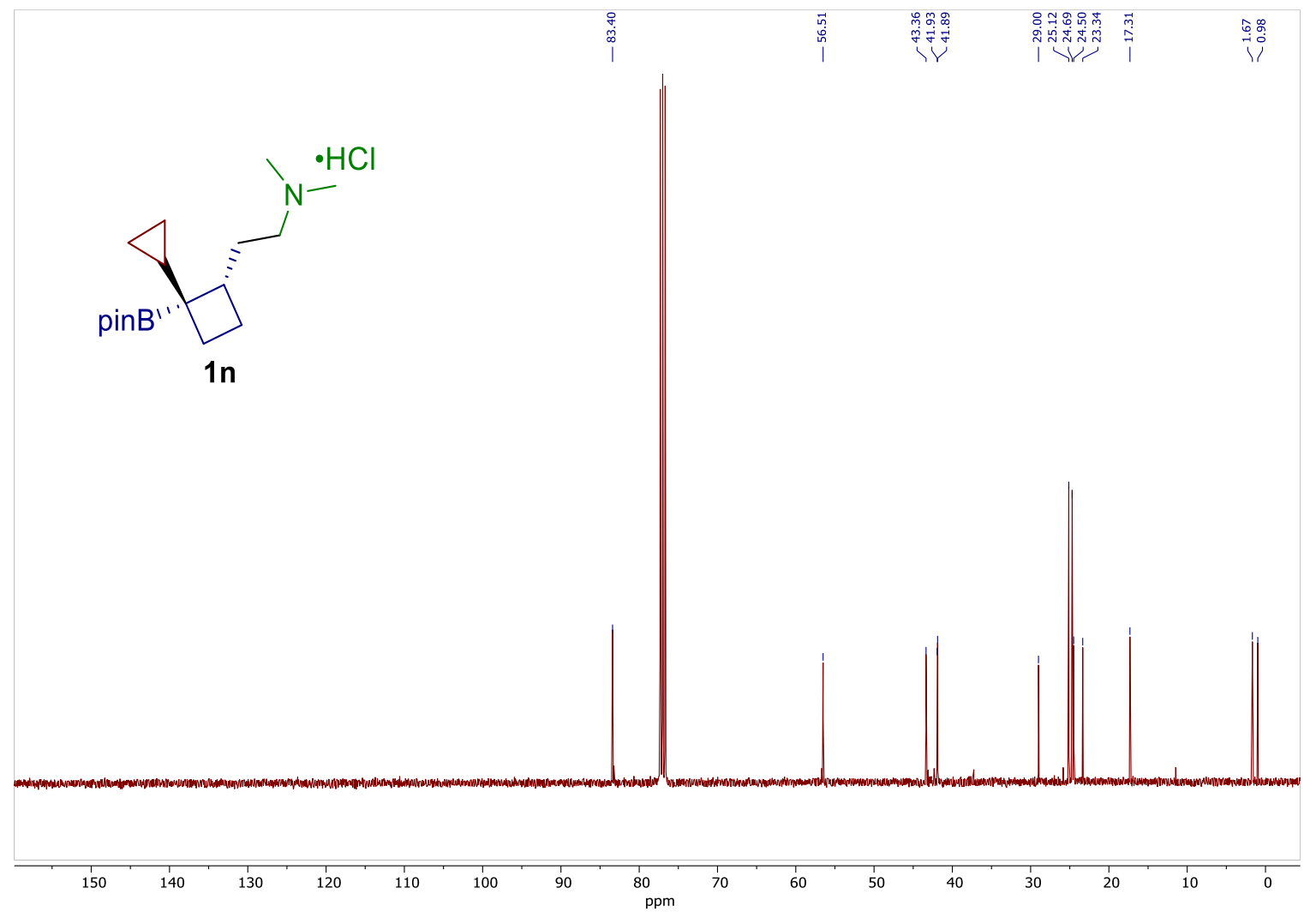


${ }^{1} \mathbf{H}-\mathbf{N M R}\left(400 \mathrm{MHz}, \mathrm{CDCl}_{3}\right)$ of compound 10 see procedure

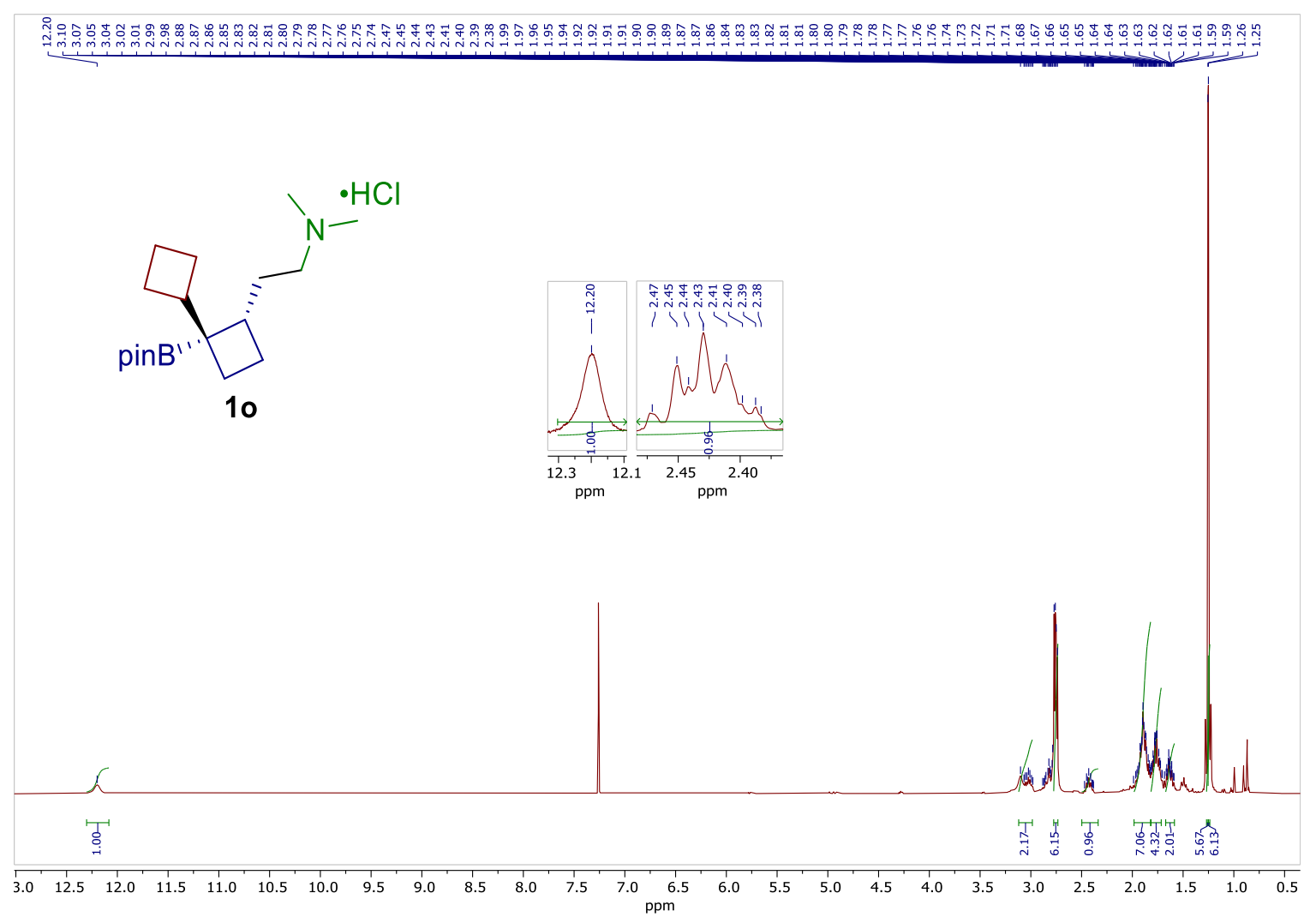

${ }^{13} \mathbf{C}-\mathbf{N M R}\left(100 \mathrm{MHz}, \mathrm{CDCl}_{3}\right)$ of compound $\mathbf{1 o}$

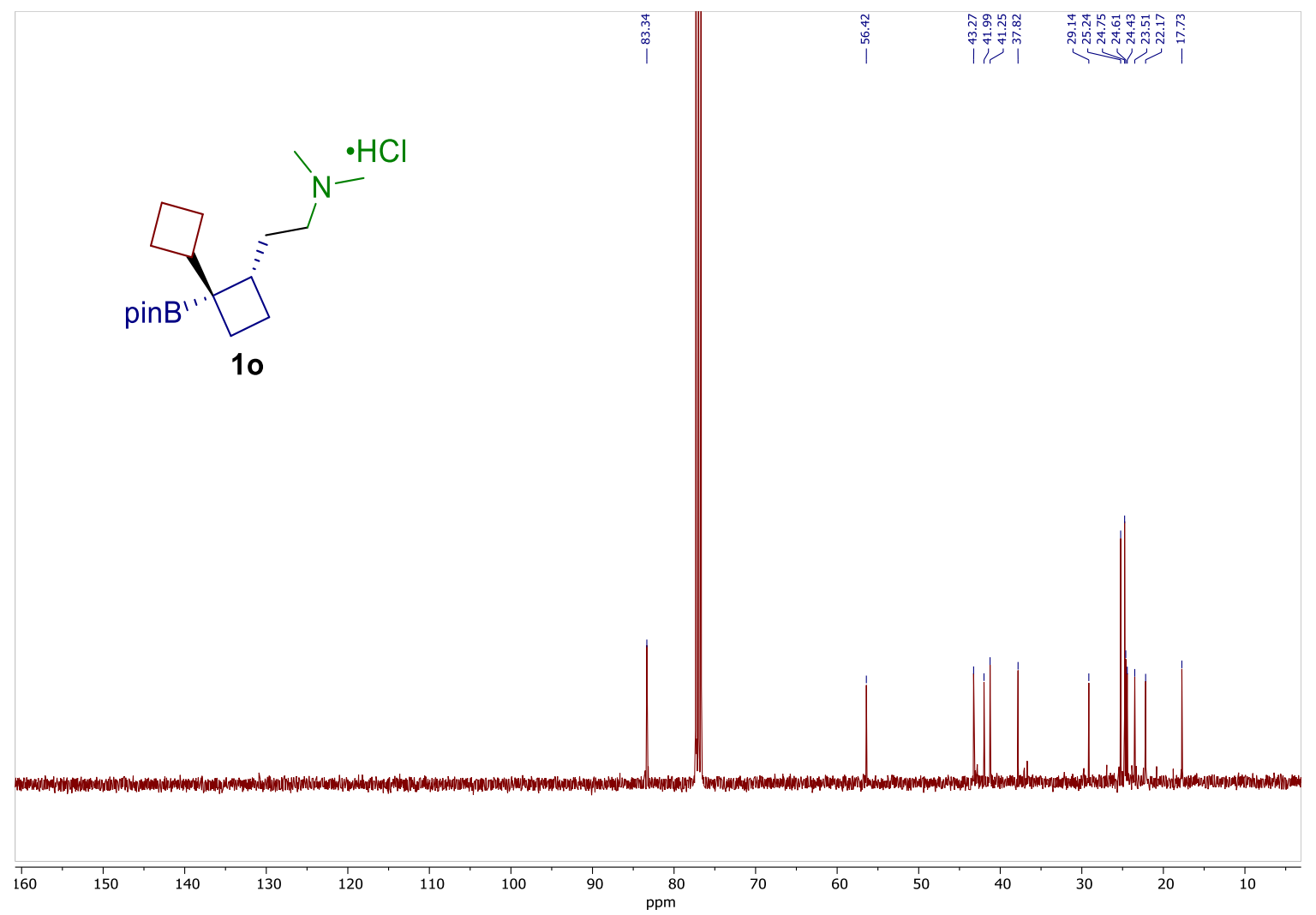


${ }^{\mathbf{1}} \mathbf{H}-\mathbf{N M R}\left(400 \mathrm{MHz}, \mathrm{CDCl}_{3}\right)$ of compound $\mathbf{1 p}$ see procedure

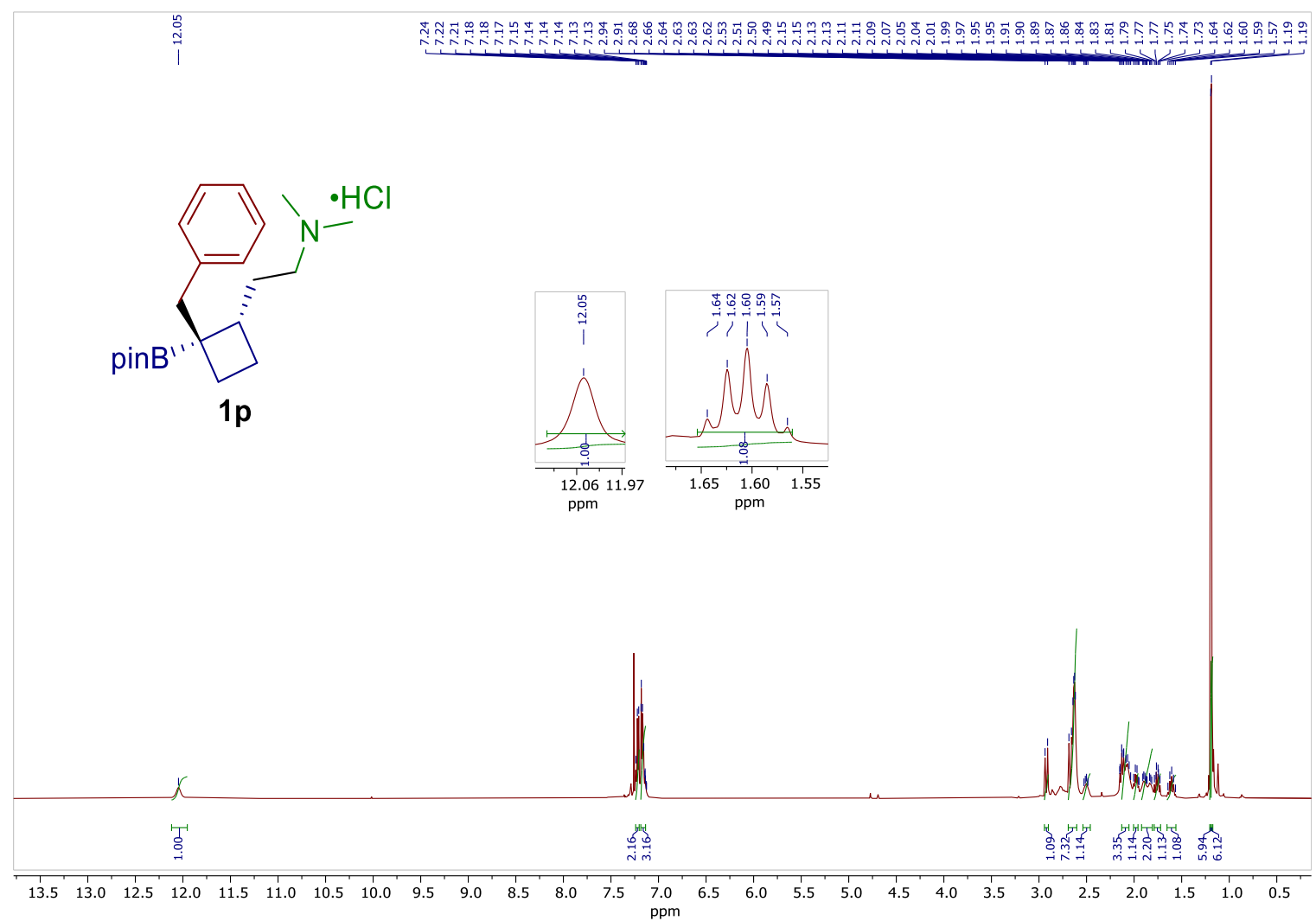

${ }^{13} \mathbf{C}$-NMR (100 MHz, $\left.\mathrm{CDCl}_{3}\right)$ of compound 1p

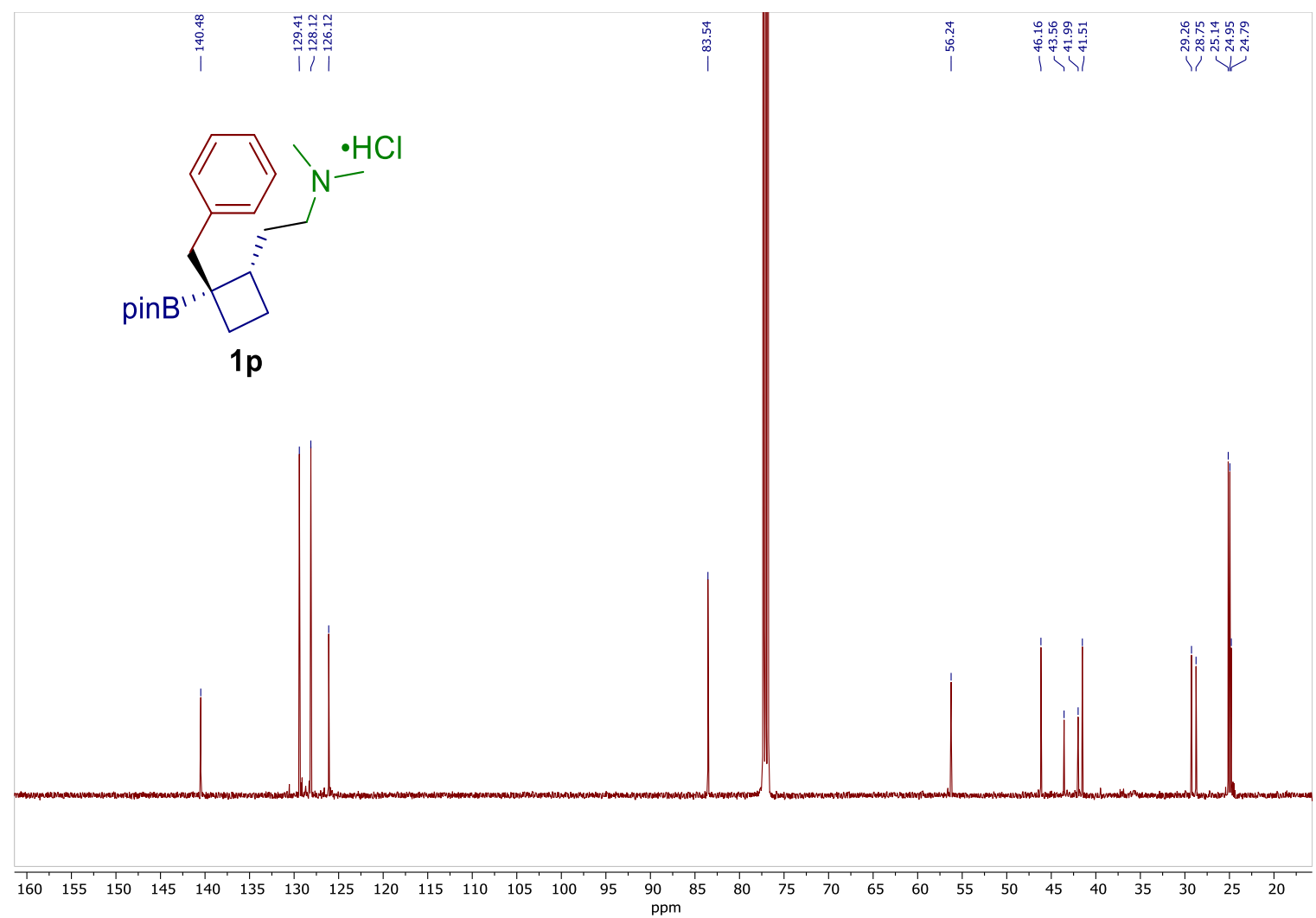


${ }^{1} \mathbf{H}-\mathrm{NMR}\left(400 \mathrm{MHz}, \mathrm{CDCl}_{3}\right)$ of compound $\mathbf{1 q}$ see procedure

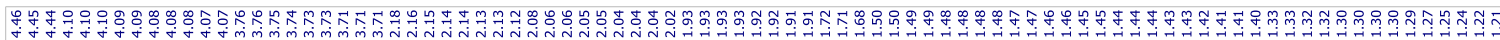
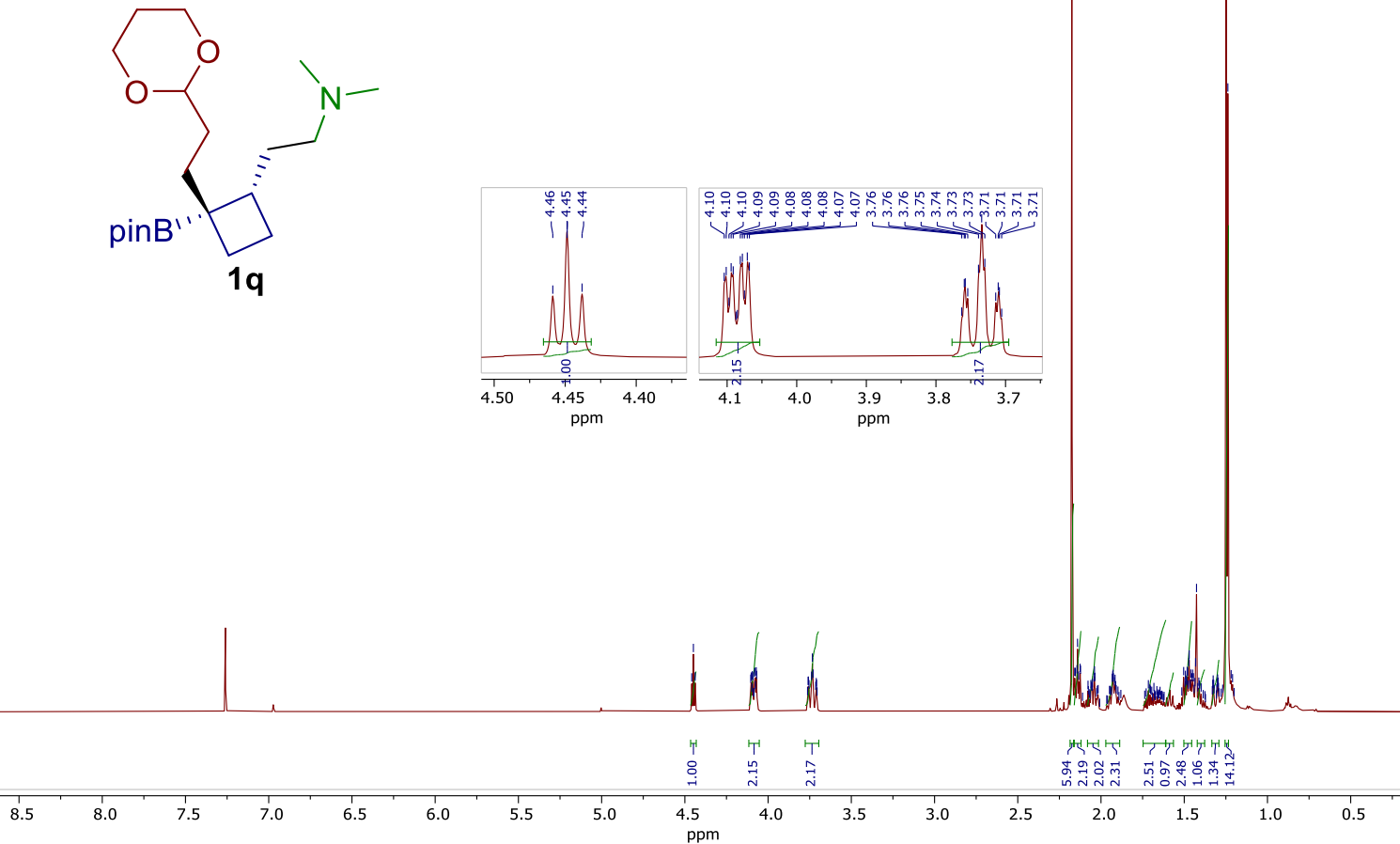

${ }^{13} \mathbf{C}-\mathbf{N M R}\left(100 \mathrm{MHz}, \mathrm{CDCl}_{3}\right)$ of compound $\mathbf{1 q}$

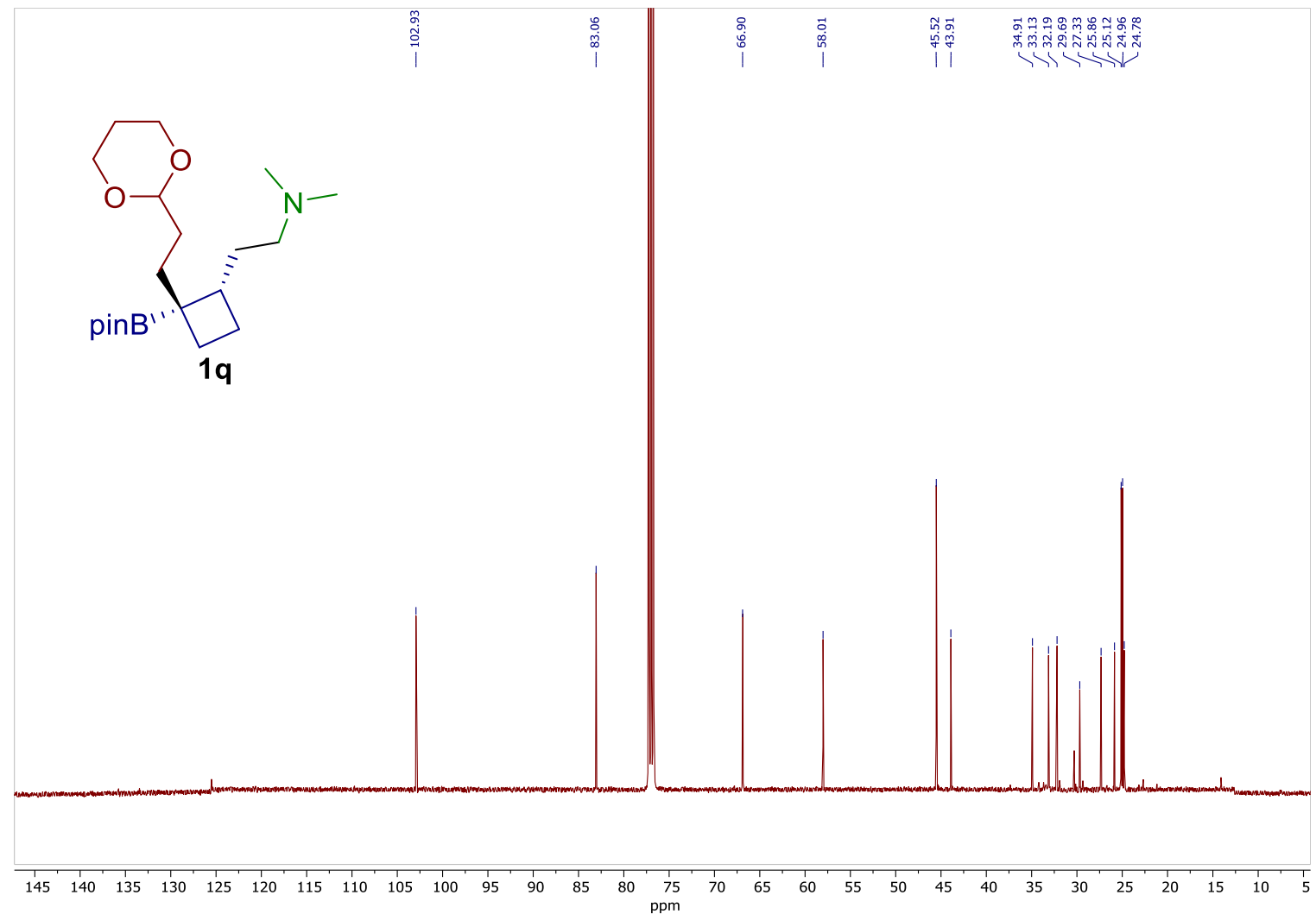


${ }^{1} \mathbf{H}-\mathbf{N M R}\left(400 \mathrm{MHz}, \mathrm{CDCl}_{3}\right)$ of compound $\mathbf{1 r}$ see procedure

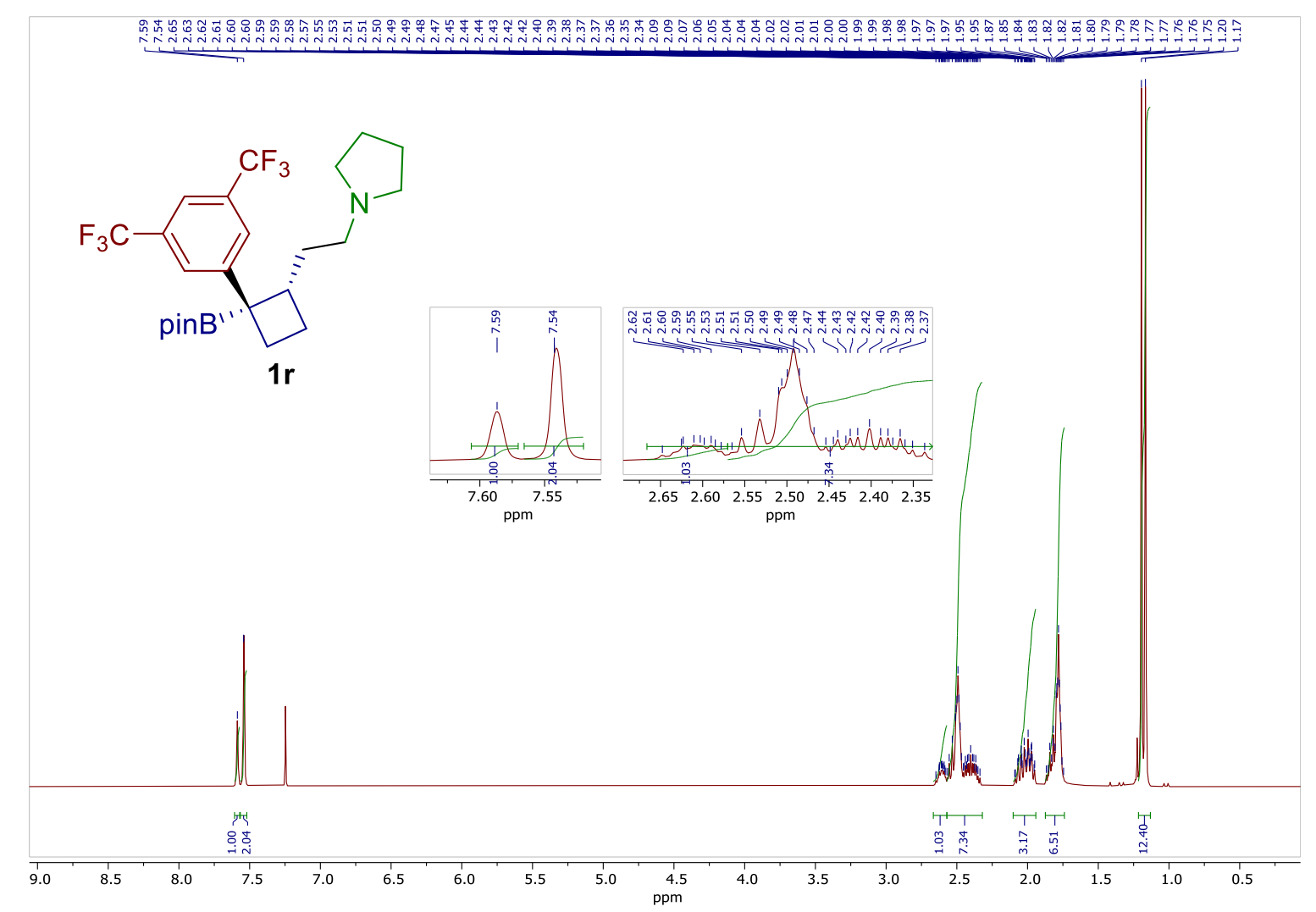

${ }^{13} \mathbf{C}-\mathbf{N M R}\left(100 \mathrm{MHz}, \mathrm{CDCl}_{3}\right)$ of compound $\mathbf{1 r}$

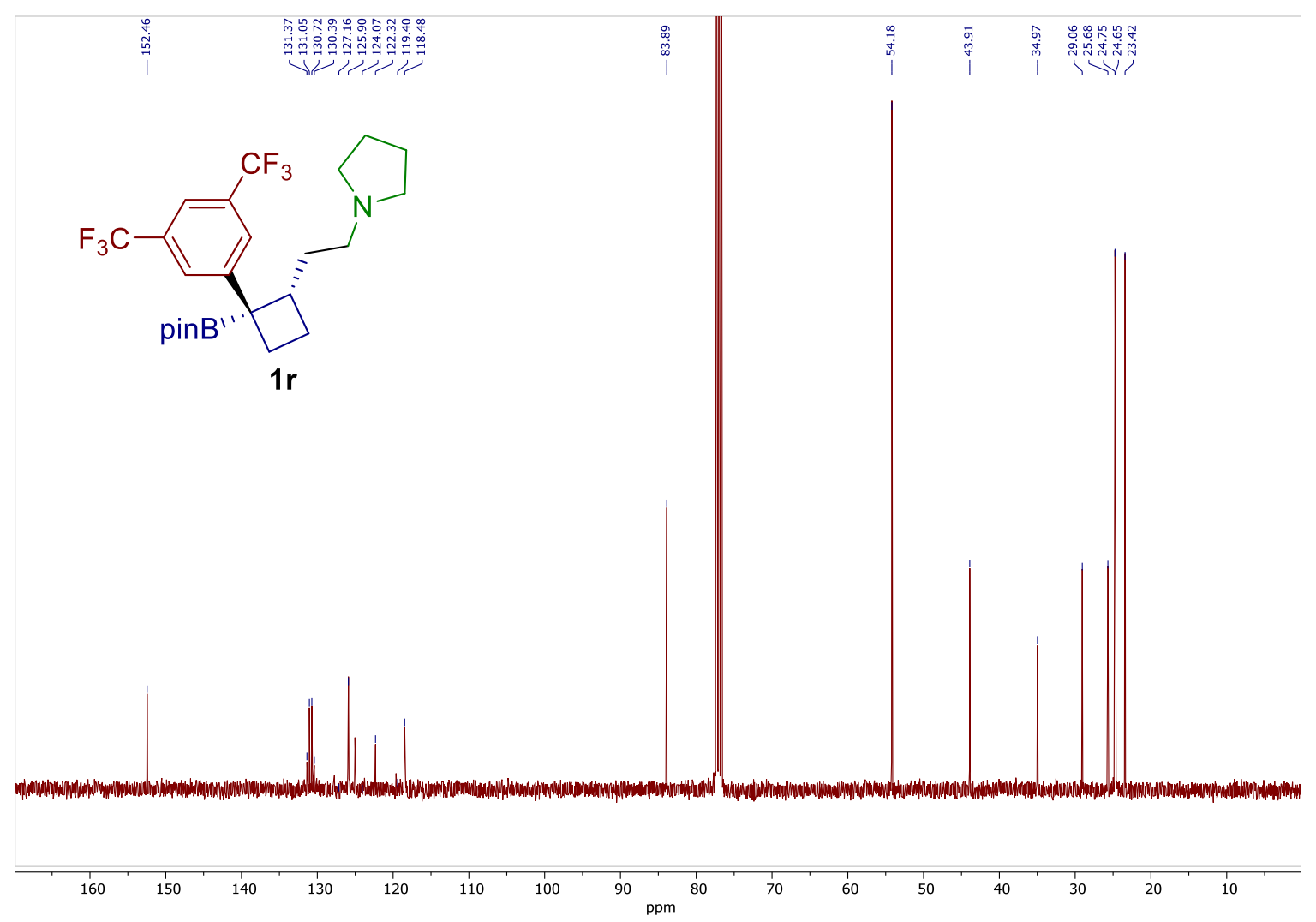


${ }^{19} \mathbf{F}-\mathbf{N M R}\left(376 \mathrm{MHz}, \mathrm{CDCl}_{3}\right)$ of compound 1r

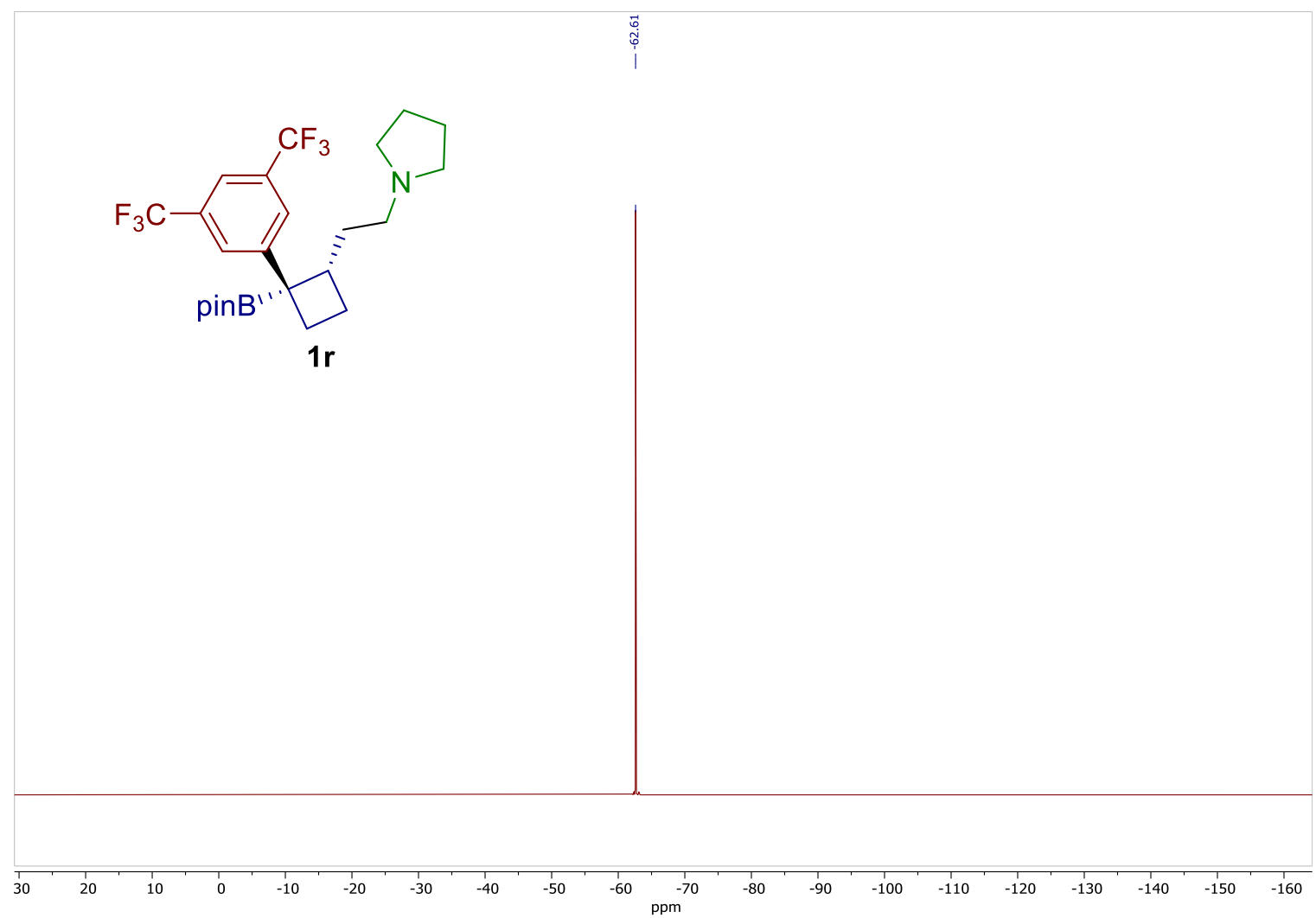


${ }^{1} \mathbf{H}-\mathbf{N M R}\left(400 \mathrm{MHz}, \mathrm{CDCl}_{3}\right)$ of compound $\mathbf{1 s}$ see procedure

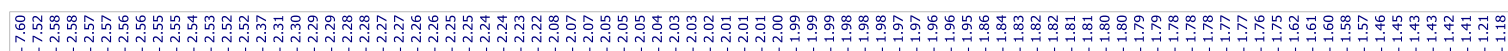<smiles></smiles>
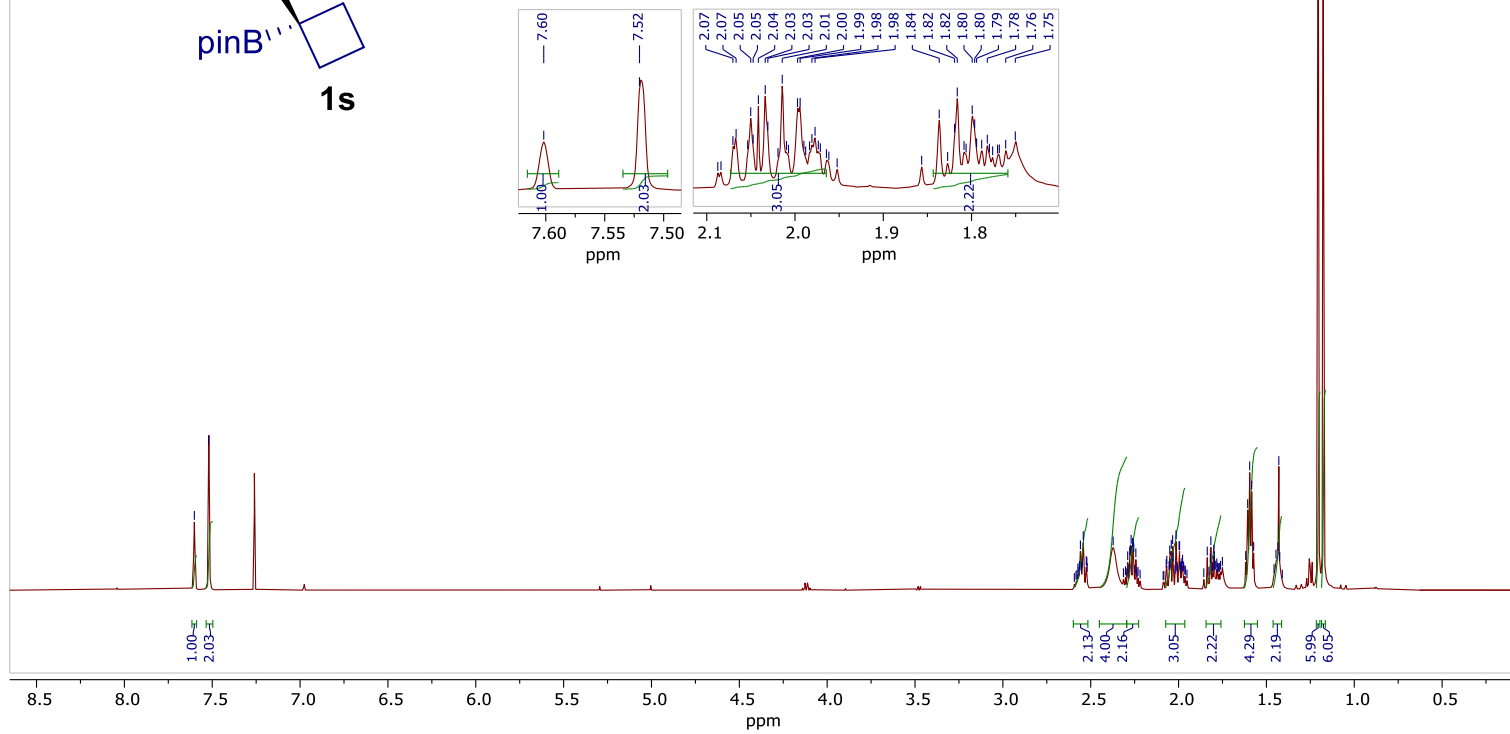

${ }^{13} \mathbf{C}-\mathbf{N M R}\left(100 \mathrm{MHz}, \mathrm{CDCl}_{3}\right)$ of compound $\mathbf{1 s}$

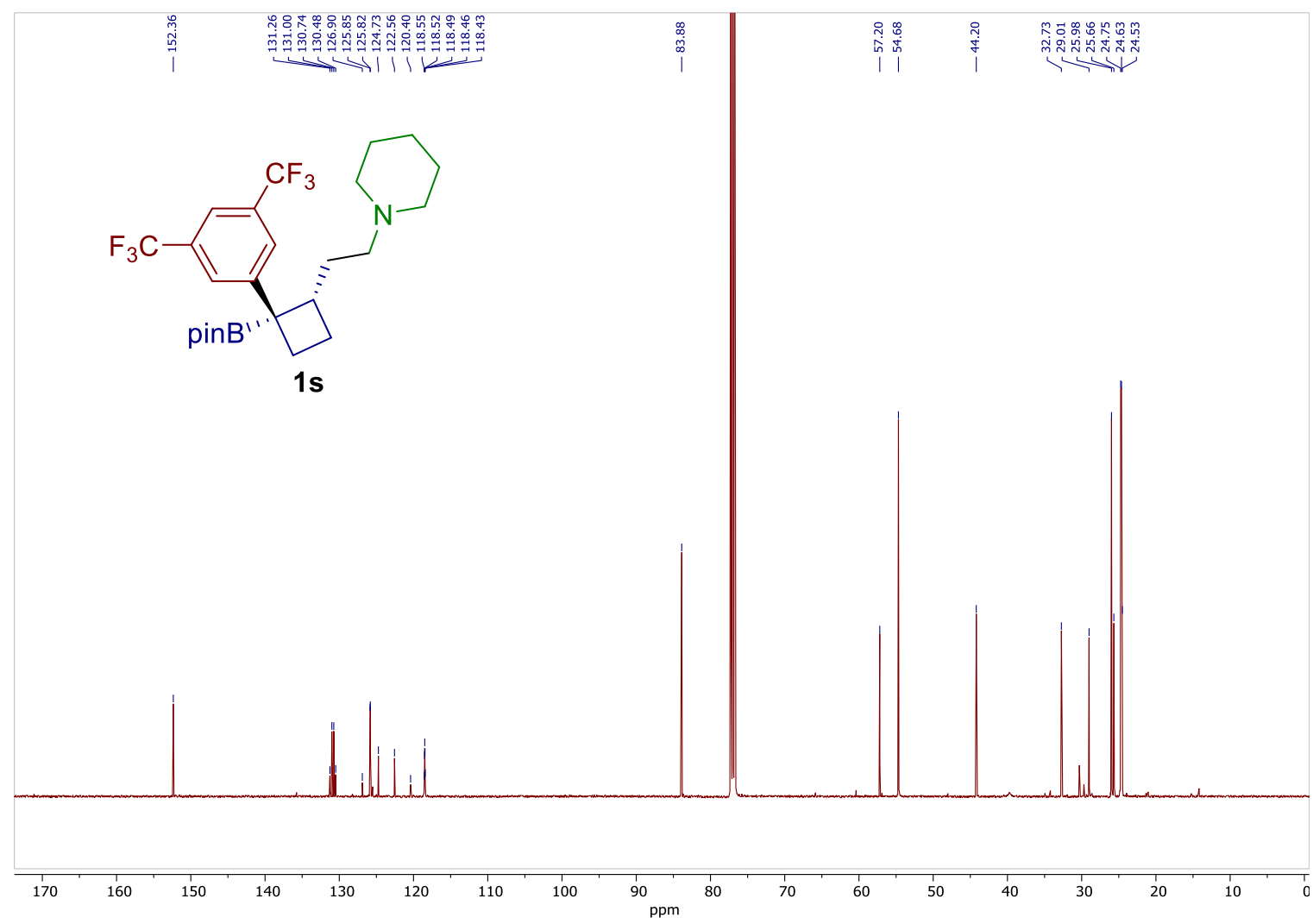


${ }^{19} \mathbf{F}-\mathbf{N M R}\left(376 \mathrm{MHz}, \mathrm{CDCl}_{3}\right)$ of compound $\mathbf{1 s}$

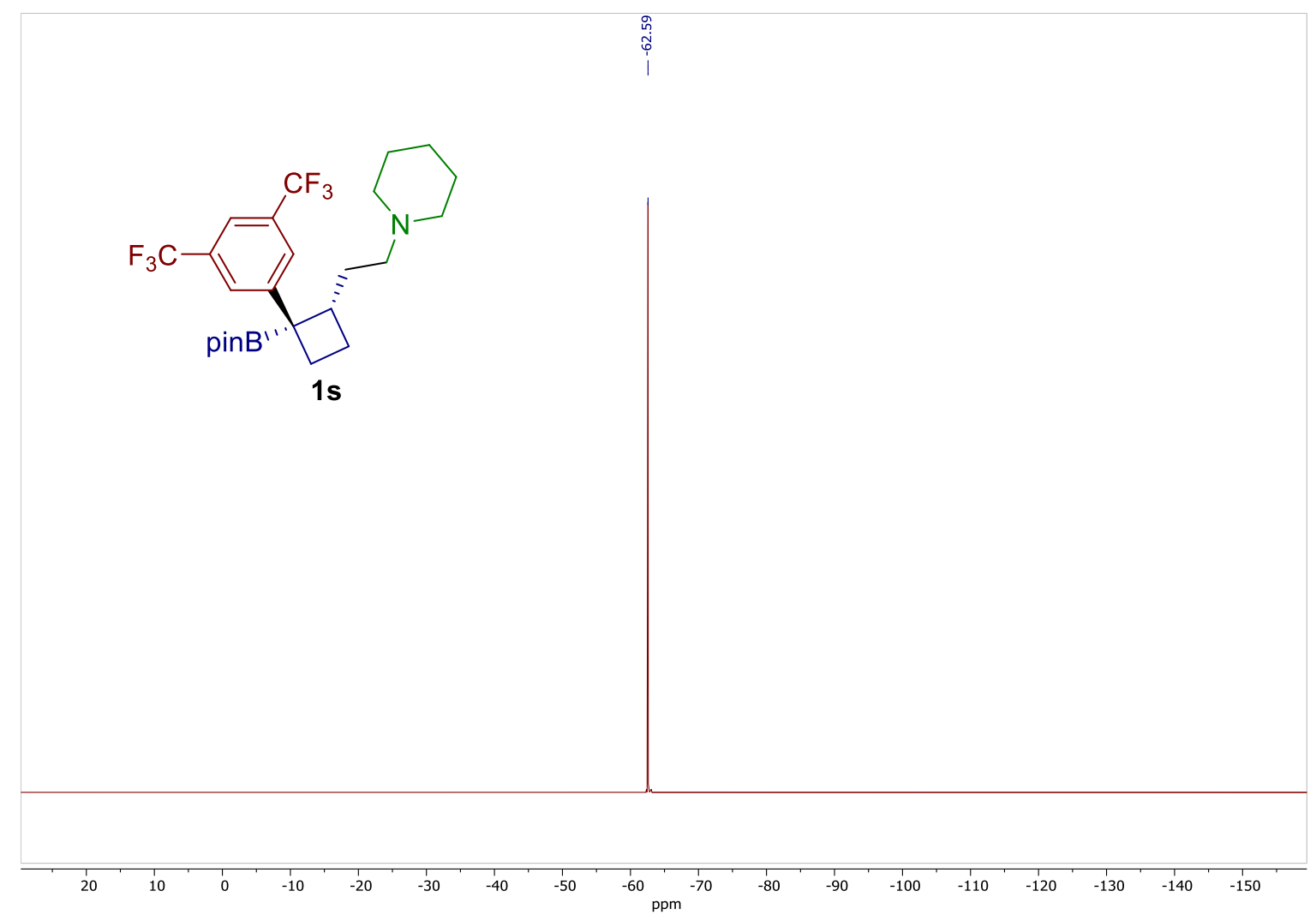


${ }^{1} \mathbf{H}-\mathbf{N M R}\left(400 \mathrm{MHz}, \mathrm{CDCl}_{3}\right)$ of compound $\mathbf{1 t}$ see procedure

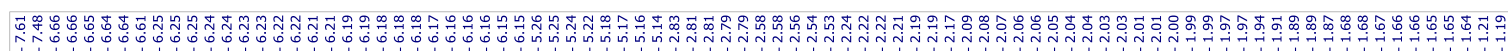<smiles>FC(F)(F)c1cc(C(F)(F)F)cc([C@@]2([10B][Pb])CC[C@H]2CC2C=CC=CC=C2)c1</smiles>
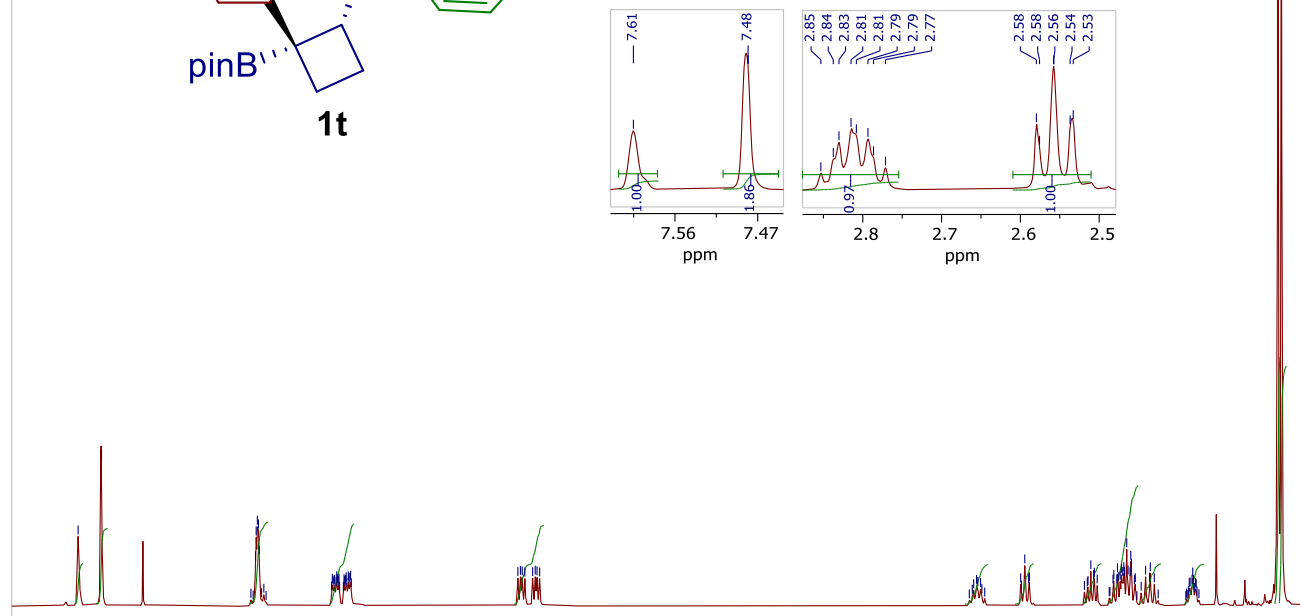

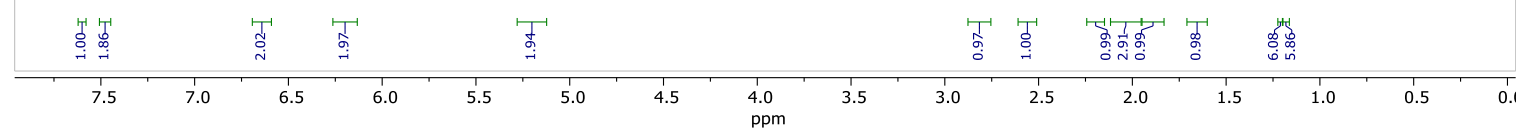

${ }^{13} \mathbf{C}$-NMR (100 MHz, $\left.\mathrm{CDCl}_{3}\right)$ of compound $\mathbf{1 t}$

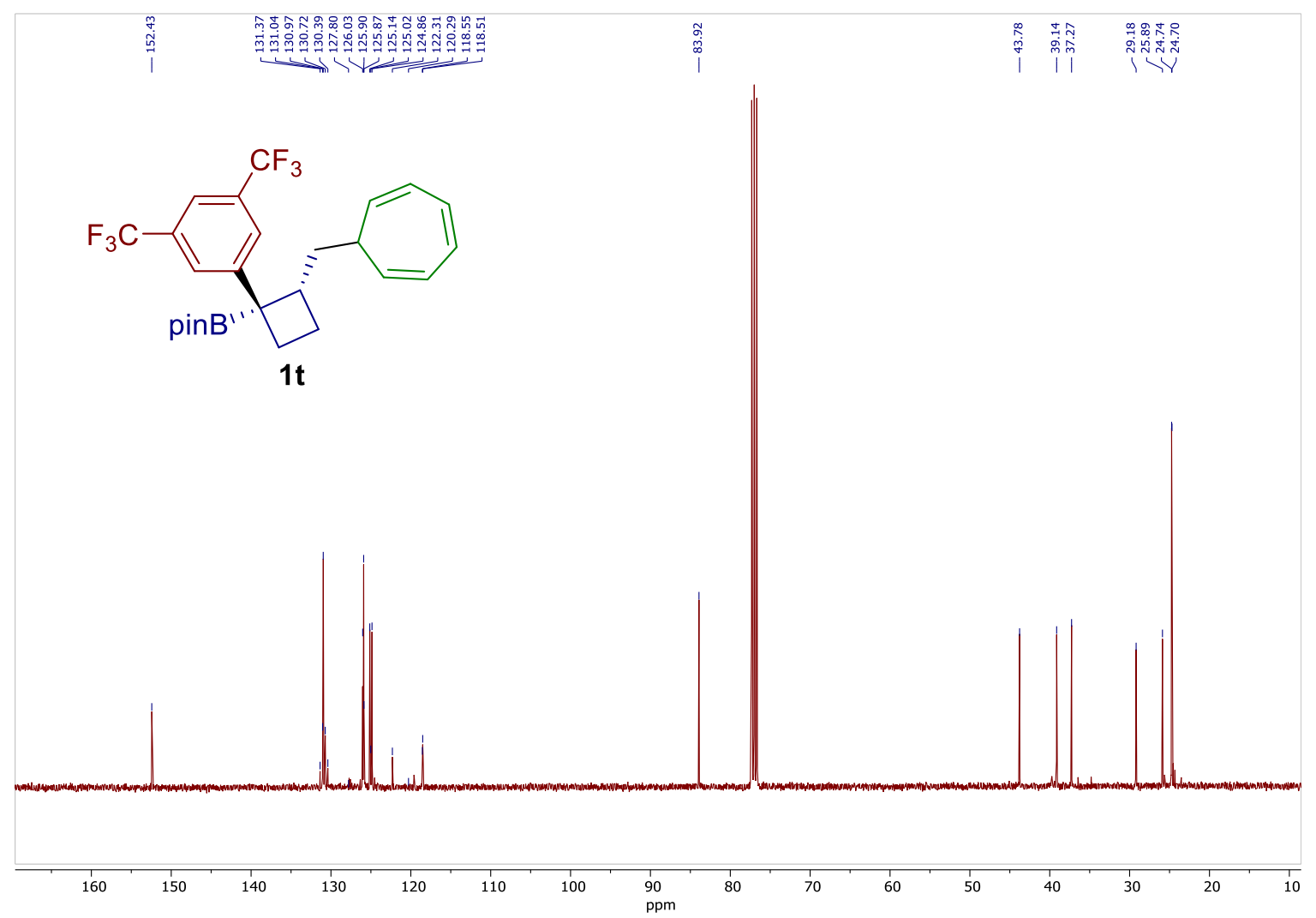


${ }^{19}$ F-NMR $\left(376 \mathrm{MHz}, \mathrm{CDCl}_{3}\right)$ of compound $\mathbf{1 t}$

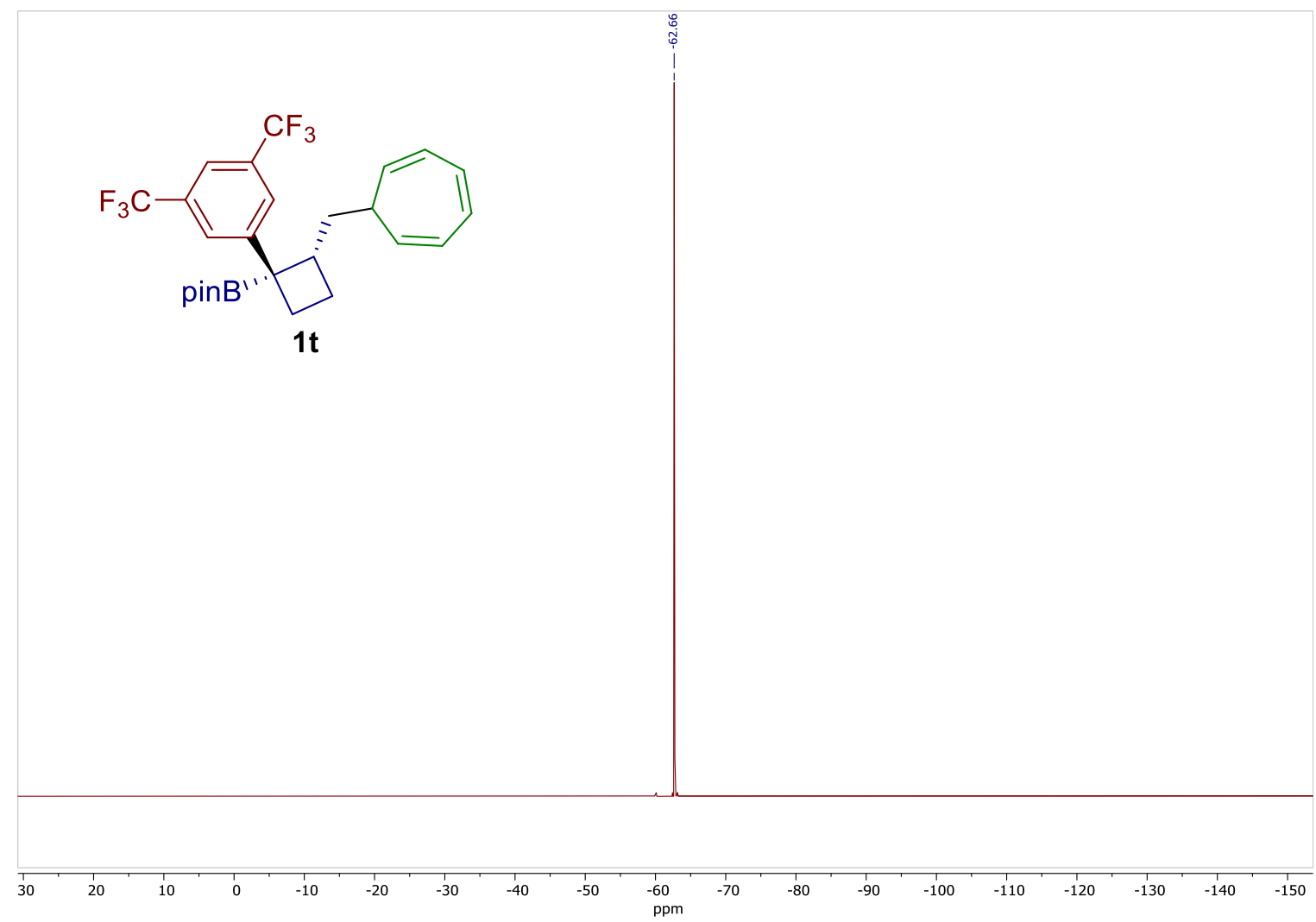


${ }^{\mathbf{1}} \mathbf{H}-\mathbf{N M R}\left(400 \mathrm{MHz}, \mathrm{CDCl}_{3}\right)$ of compound $\mathbf{1 u}$ see procedure

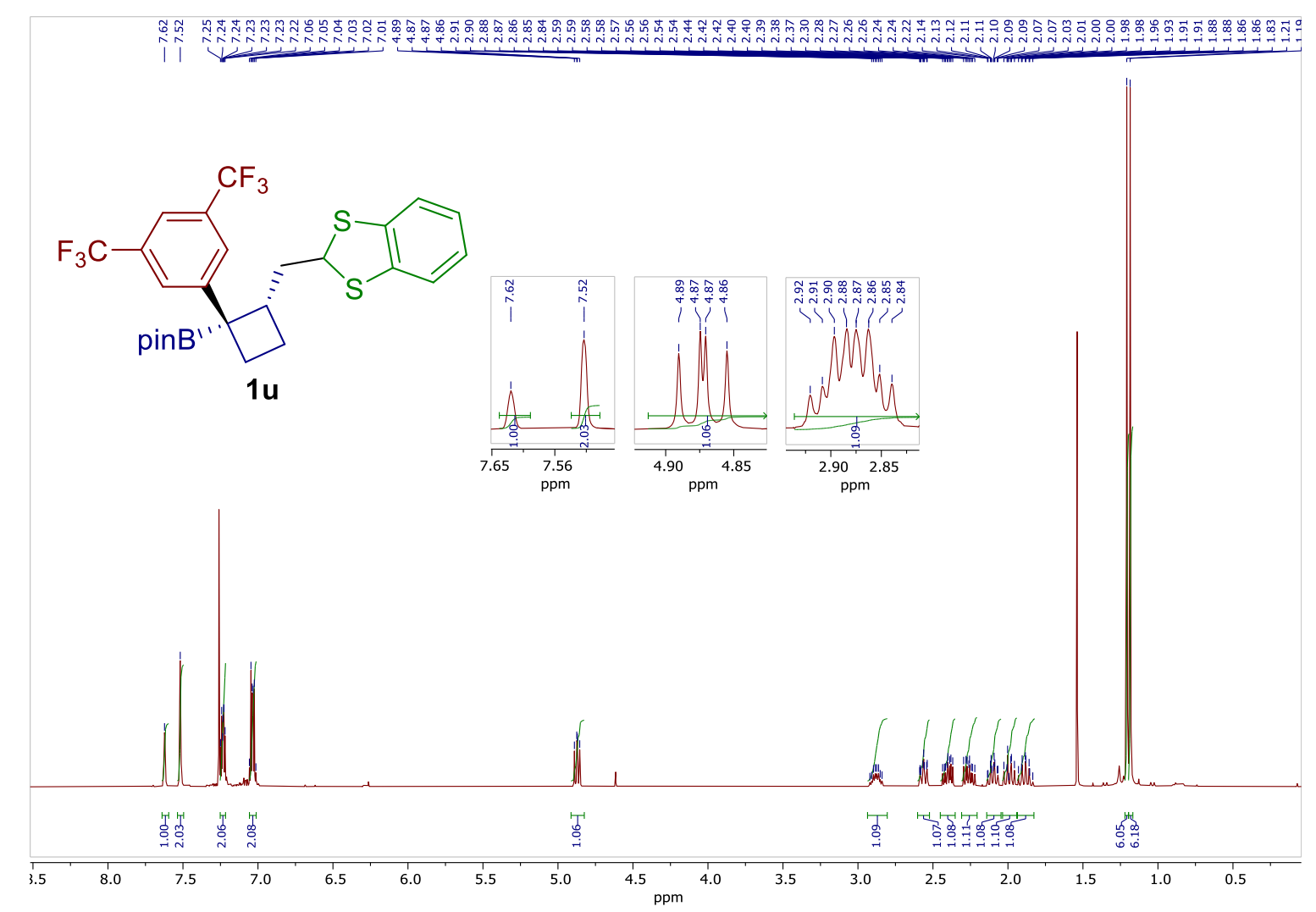

${ }^{13} \mathbf{C}$-NMR (100 MHz, $\left.\mathrm{CDCl}_{3}\right)$ of compound $\mathbf{1 u}$

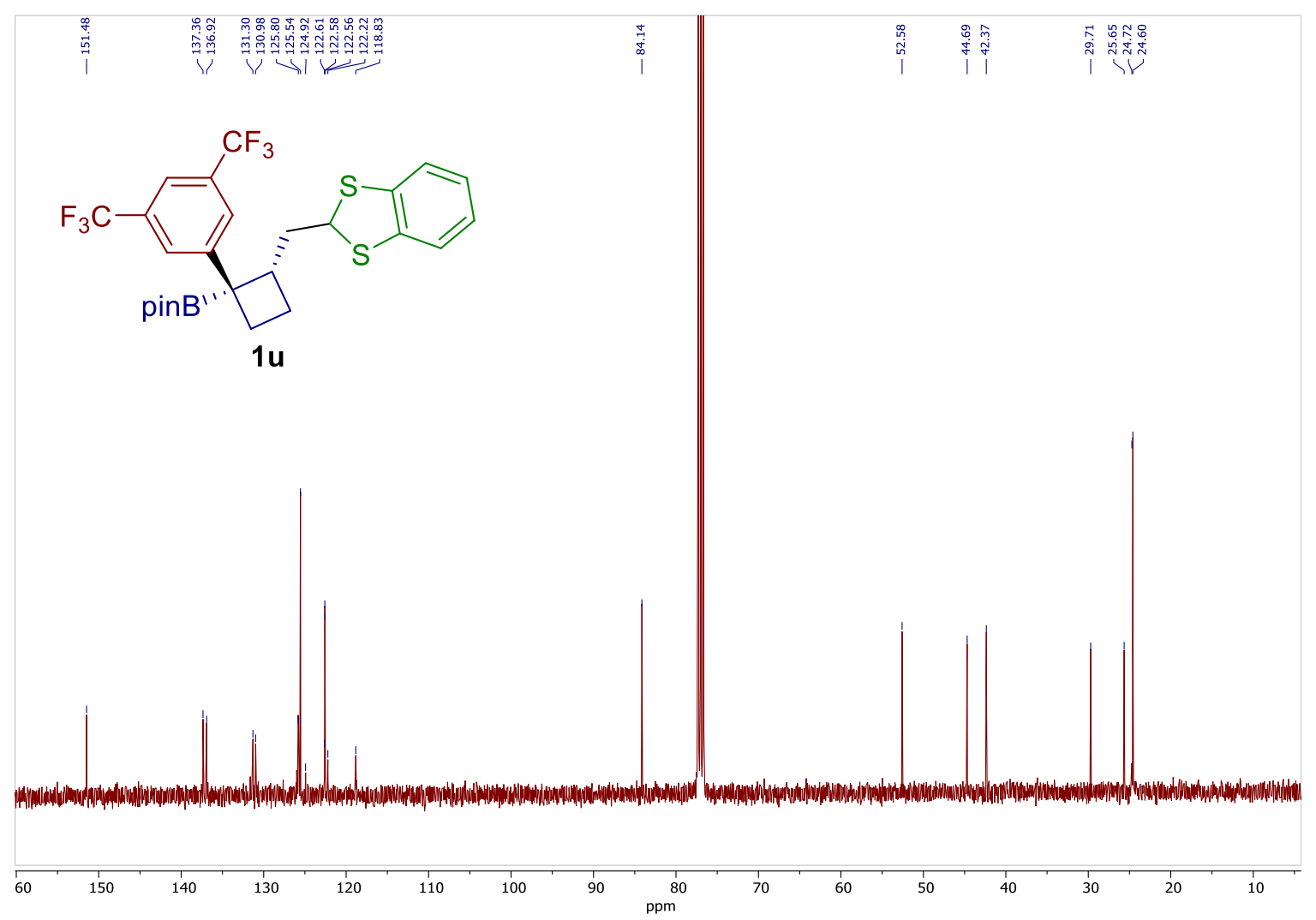


${ }^{19} \mathbf{F}-\mathbf{N M R}\left(376 \mathrm{MHz}, \mathrm{CDCl}_{3}\right)$ of compound $\mathbf{1 u}$

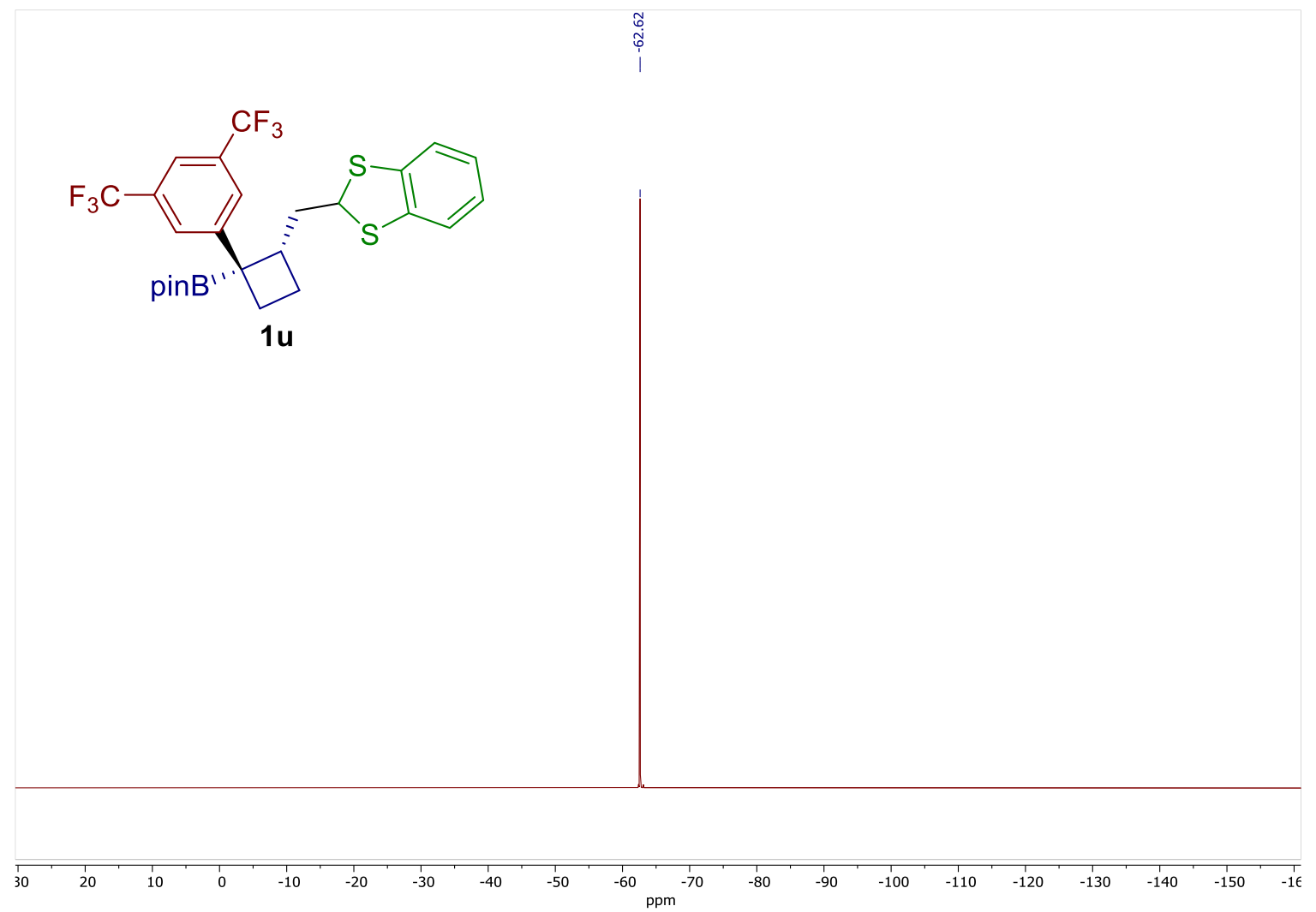


${ }^{\mathbf{1}} \mathbf{H}-\mathbf{N M R}\left(400 \mathrm{MHz}, \mathrm{CDCl}_{3}\right.$ ) of compound $\mathbf{1 v}$ see procedure

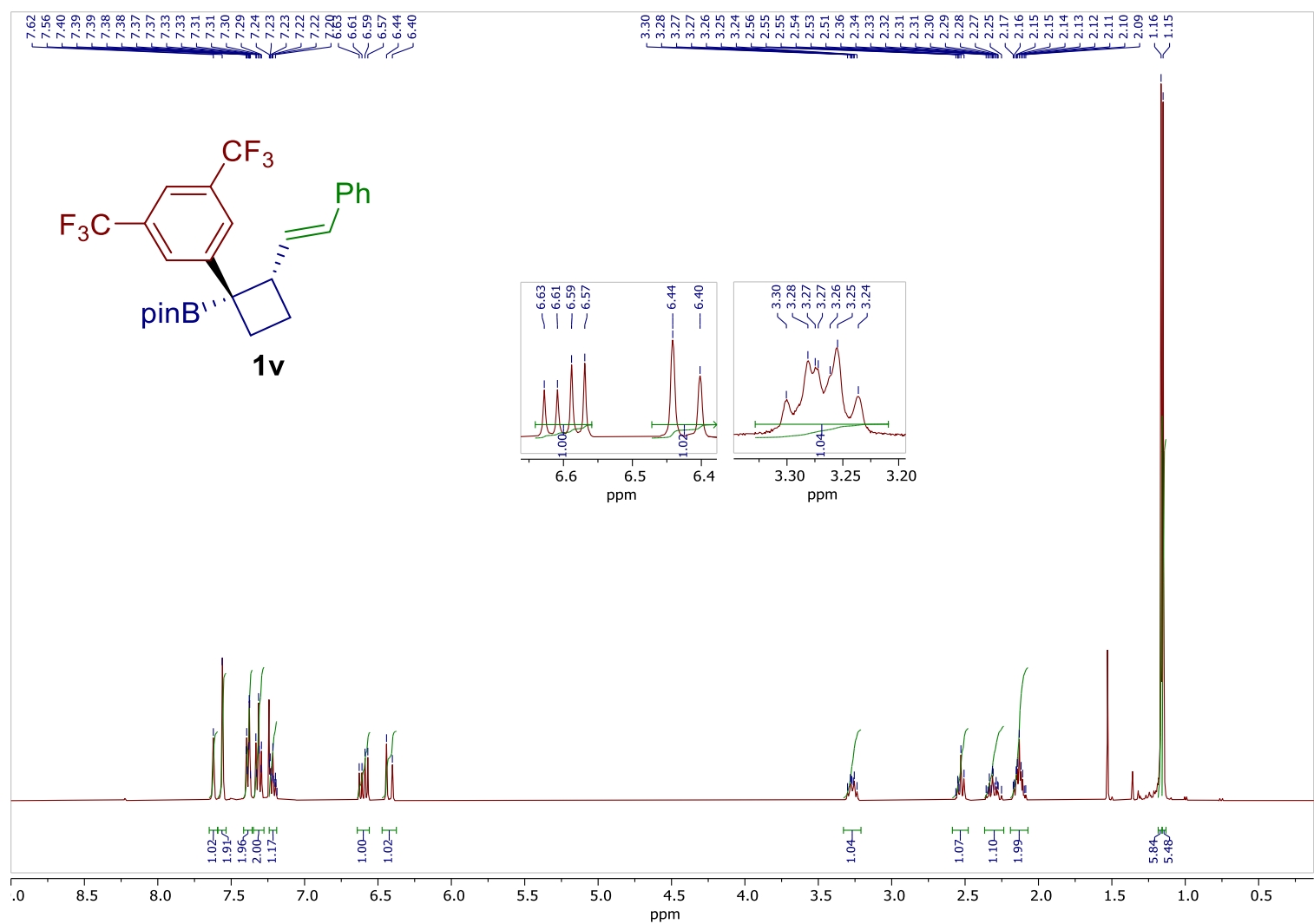

${ }^{13} \mathbf{C}$-NMR $\left(100 \mathrm{MHz}, \mathrm{CDCl}_{3}\right)$ of compound $\mathbf{1 v}$

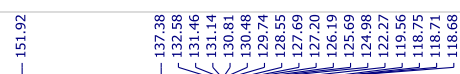
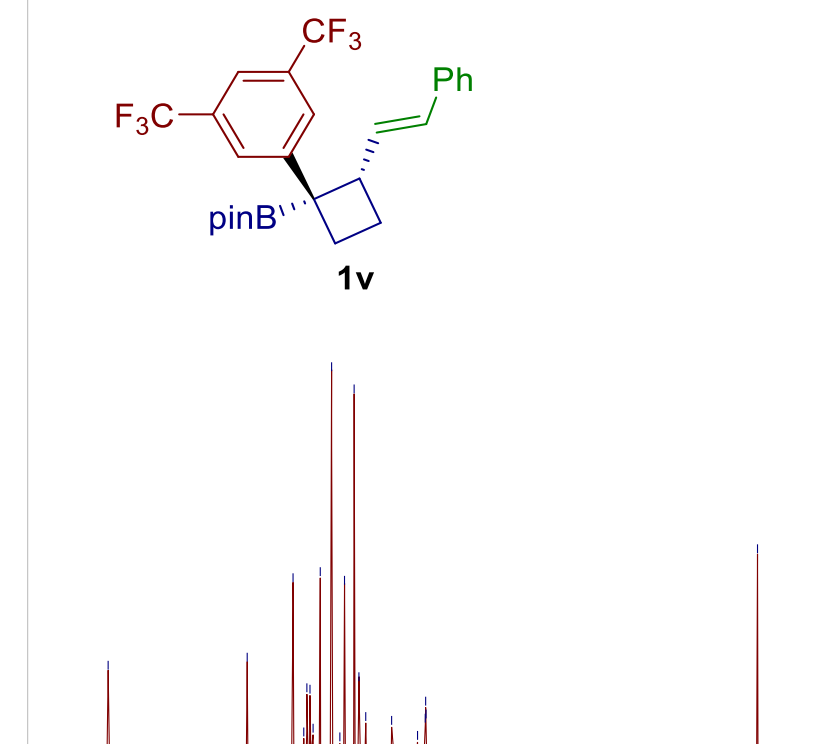

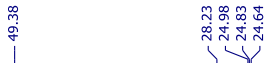

\begin{tabular}{lllllllllllllllll}
\hline 60 & 150 & 140 & 130 & 120 & 110 & 100 & 90 & $\begin{array}{c}1 \\
\mathrm{ppm}\end{array}$ & 70 & 60 & 50 & 40 & 30 & 20 & 10
\end{tabular} 
${ }^{19} \mathbf{F}-\mathbf{N M R}\left(376 \mathrm{MHz}, \mathrm{CDCl}_{3}\right)$ of compound $\mathbf{1 v}$

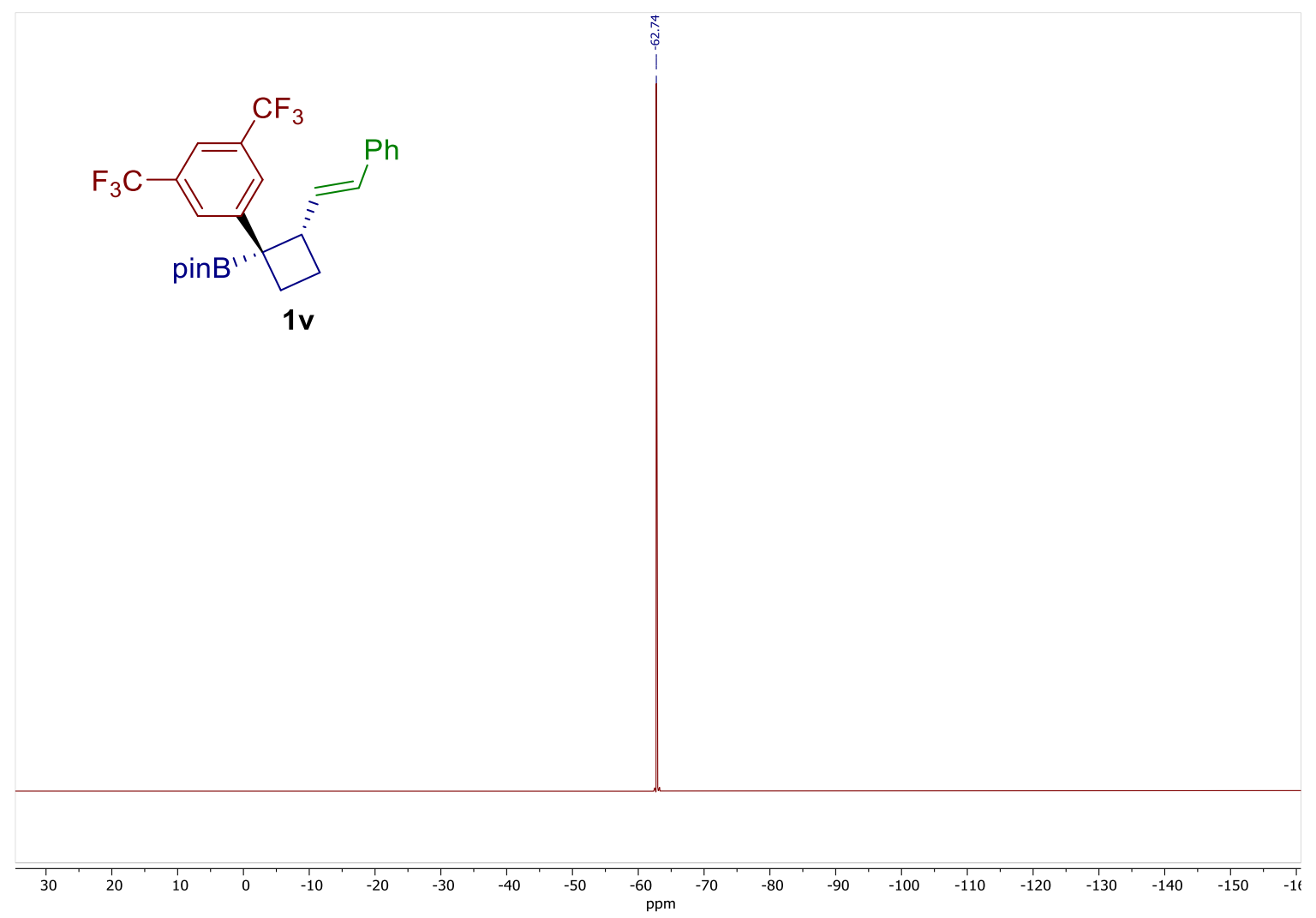


${ }^{\mathbf{1}} \mathbf{H}-\mathbf{N M R}\left(400 \mathrm{MHz}, \mathrm{CDCl}_{3}\right)$ of compound $\mathbf{1 w}$ see procedure

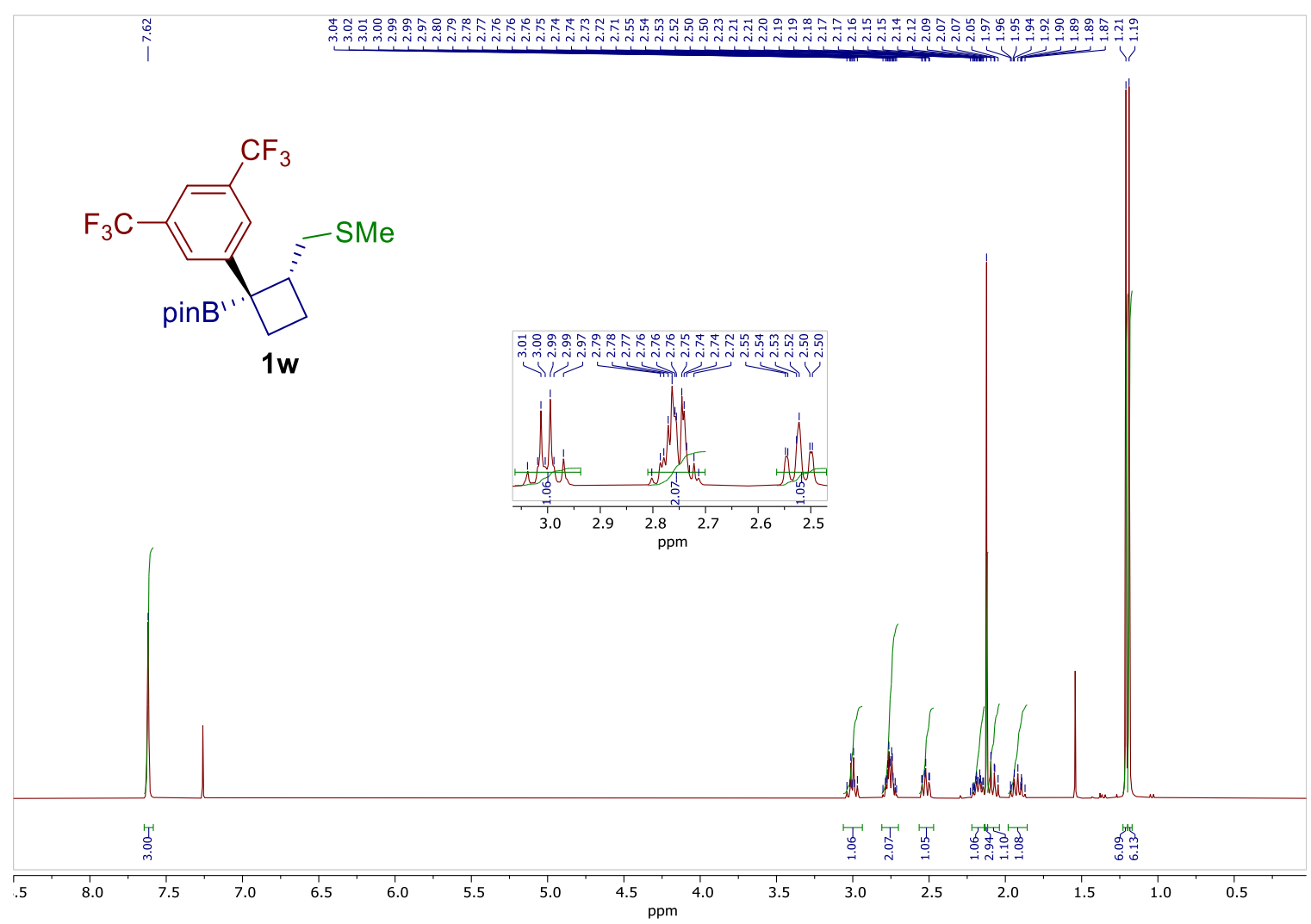

${ }^{13} \mathbf{C}$-NMR (100 MHz, $\left.\mathrm{CDCl}_{3}\right)$ of compound $\mathbf{1 w}$

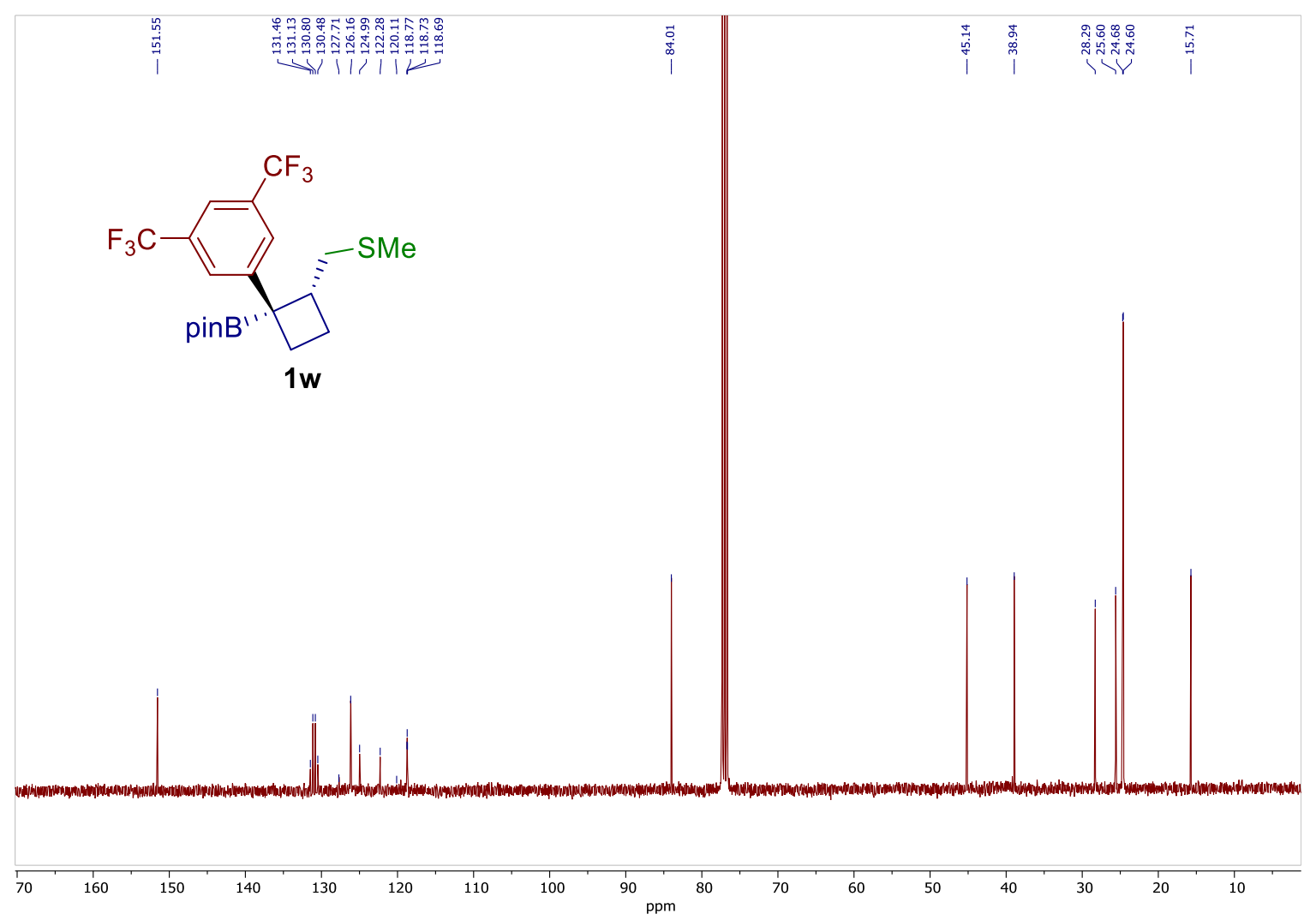


${ }^{19} \mathbf{F}-\mathbf{N M R}\left(376 \mathrm{MHz}, \mathrm{CDCl}_{3}\right)$ of compound $\mathbf{1 w}$

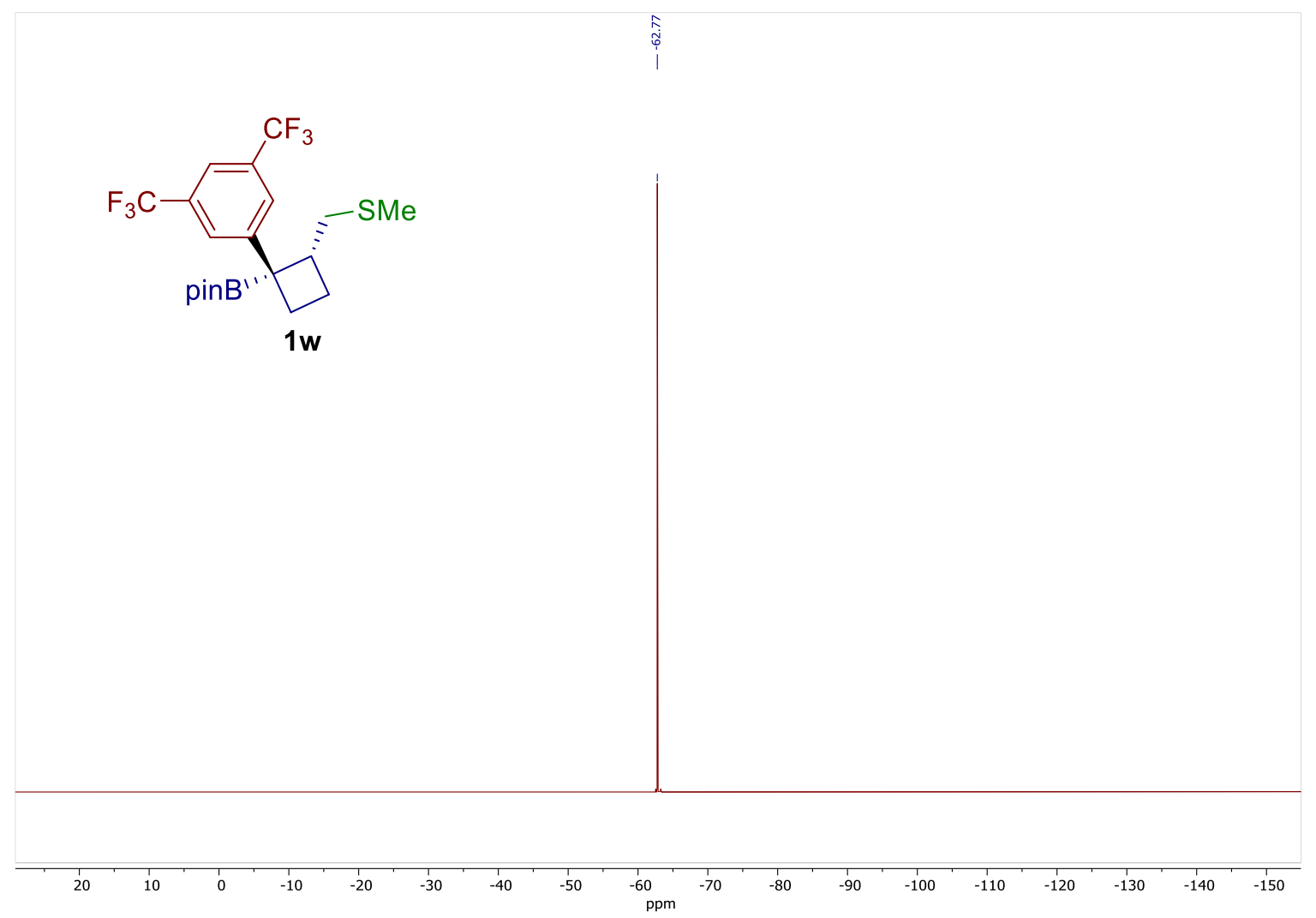


${ }^{\mathbf{1}} \mathbf{H}-\mathbf{N M R}\left(400 \mathrm{MHz}, \mathrm{CDCl}_{3}\right)$ of compound $\mathbf{1 x}$ see procedure

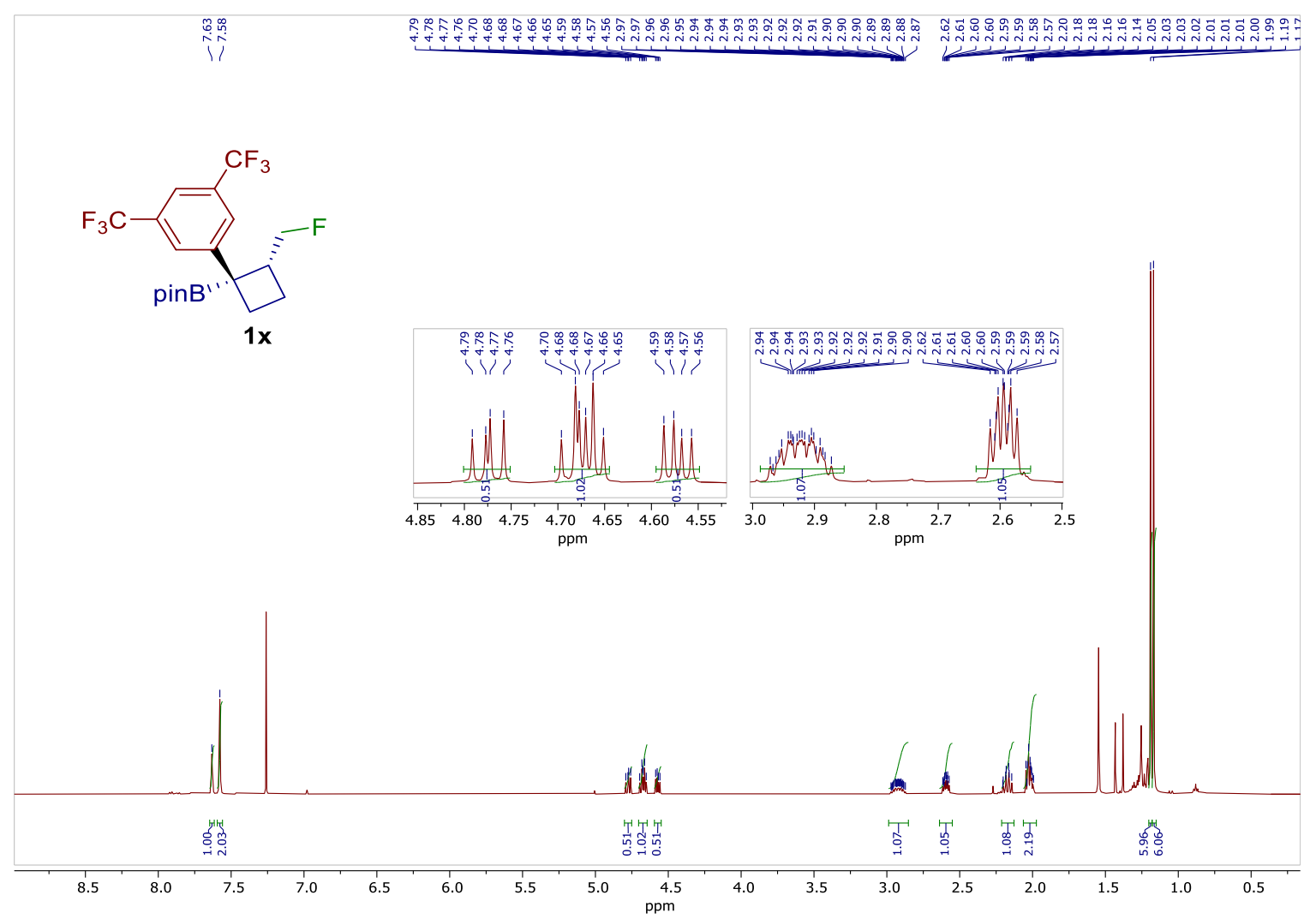

${ }^{13} \mathbf{C}$-NMR $\left(100 \mathrm{MHz}, \mathrm{CDCl}_{3}\right)$ of compound $\mathbf{1 x}$

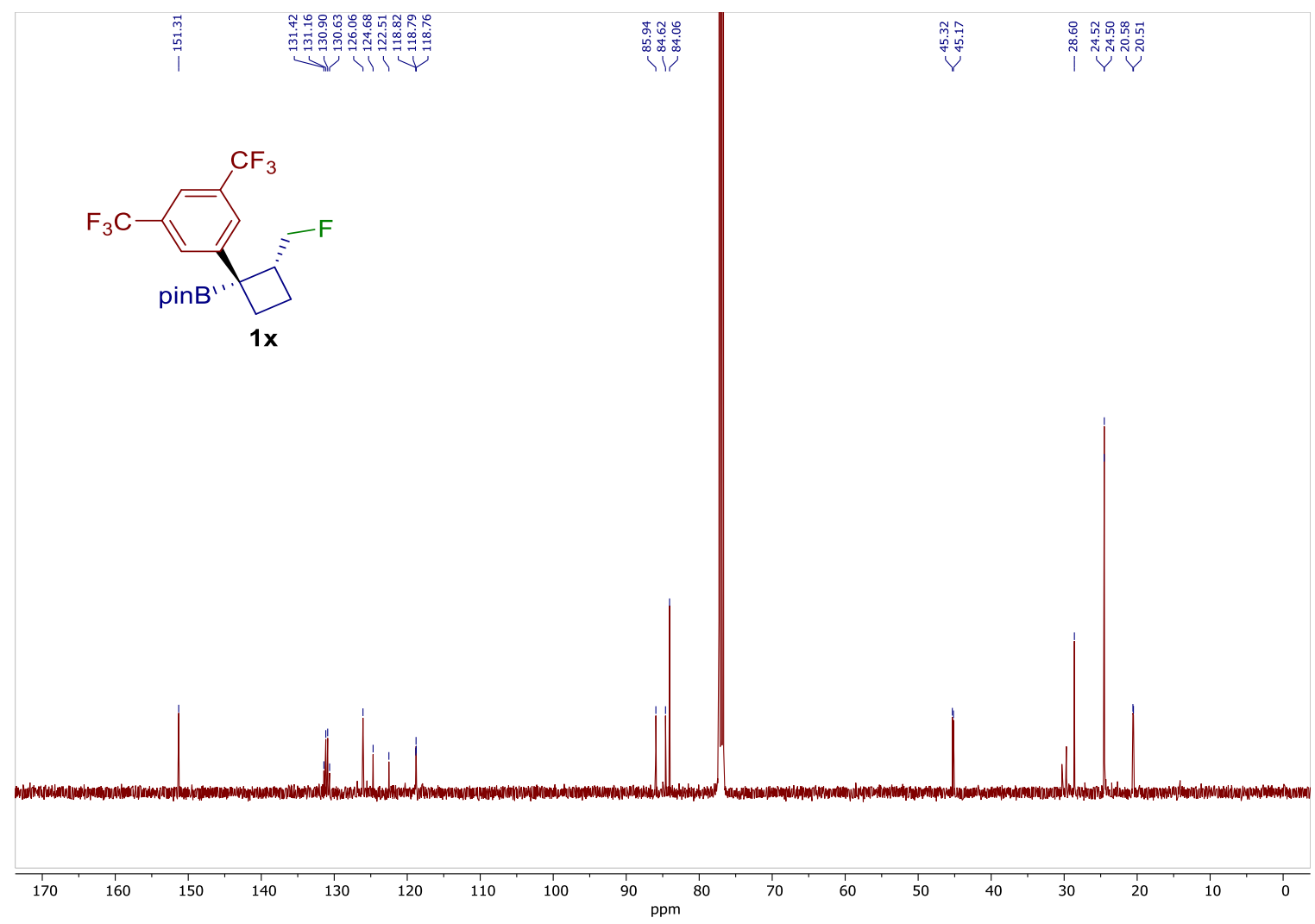


${ }^{19}$ F-NMR $\left(376 \mathrm{MHz}, \mathrm{CDCl}_{3}\right)$ of compound $\mathbf{1 x}$

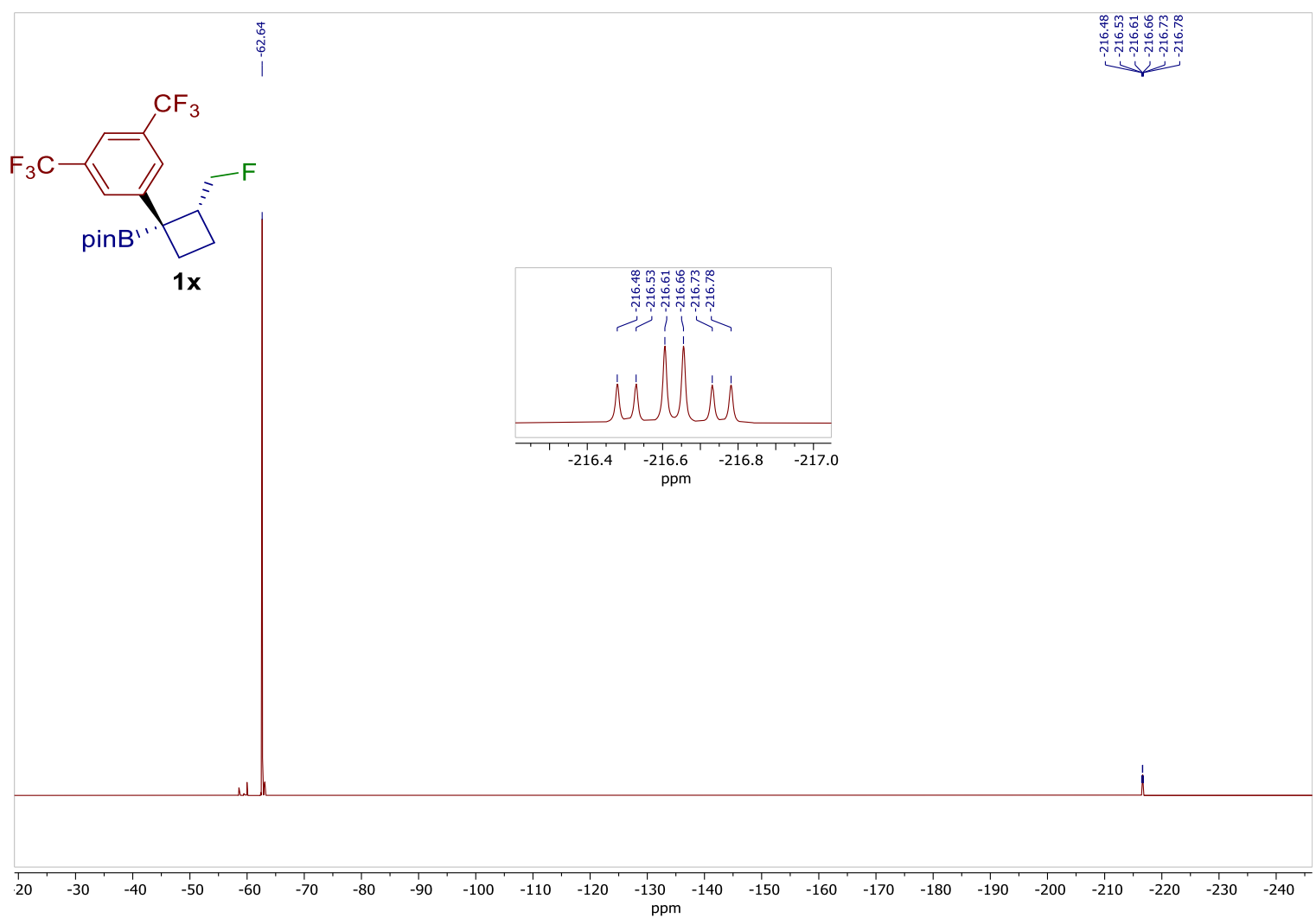


${ }^{\mathbf{1}} \mathbf{H}-\mathbf{N M R}\left(400 \mathrm{MHz}, \mathrm{CDCl}_{3}\right)$ of compound $\mathbf{1 y}$ see procedure

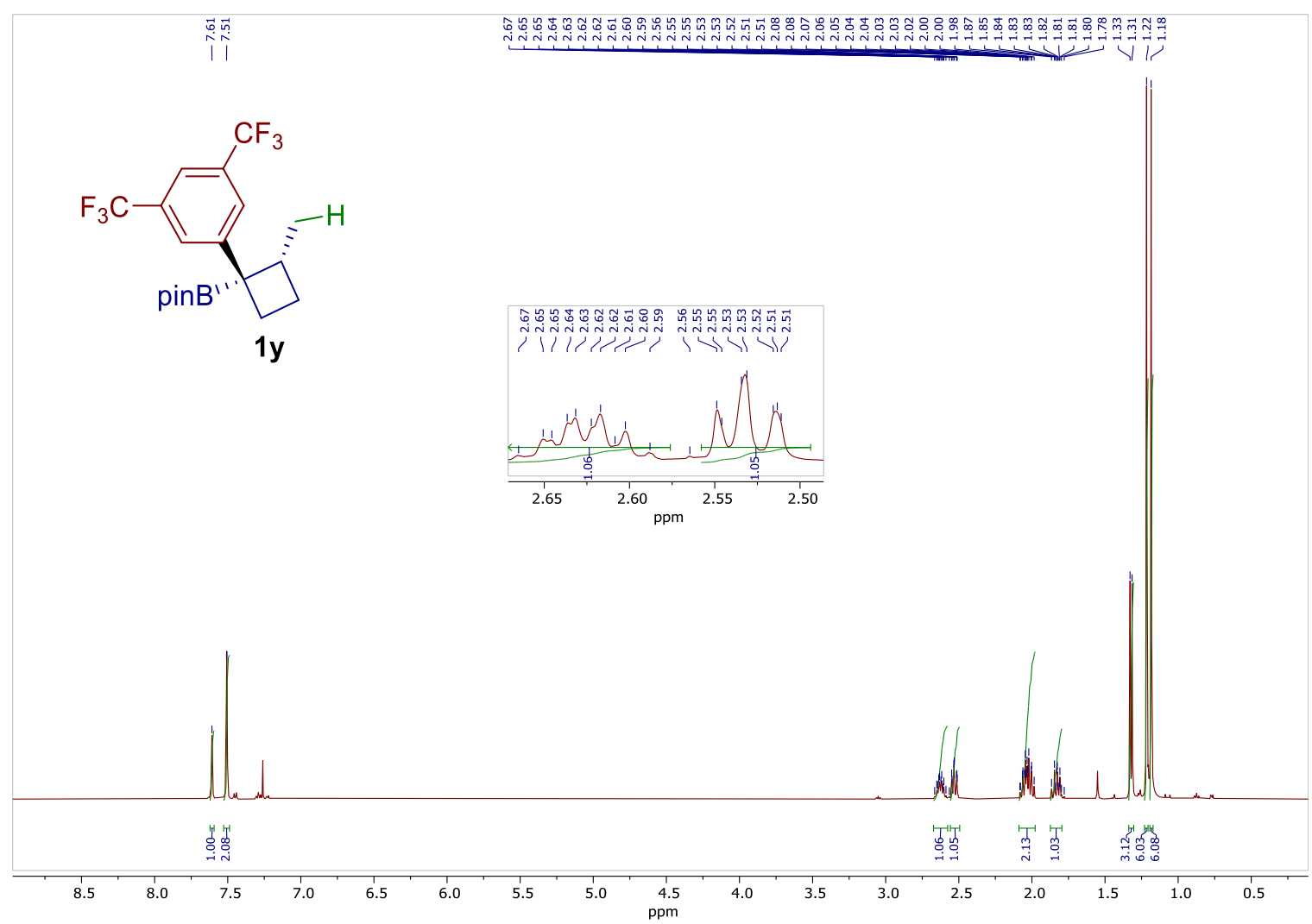

${ }^{13} \mathbf{C}-\mathbf{N M R}\left(100 \mathrm{MHz}, \mathrm{CDCl}_{3}\right)$ of compound $\mathbf{1 y}$

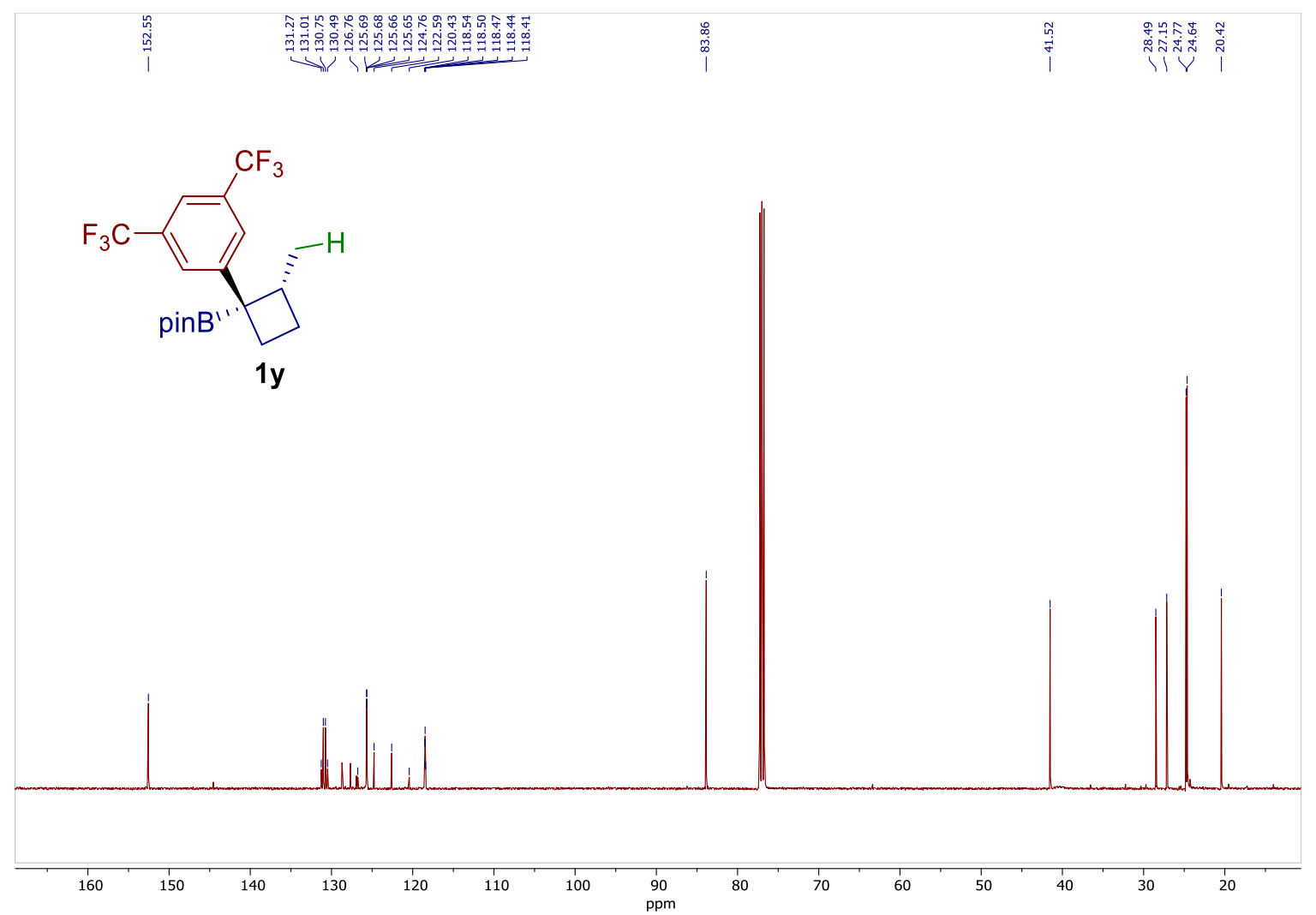


${ }^{19} \mathbf{F}-N M R\left(376 \mathrm{MHz}, \mathrm{CDCl}_{3}\right)$ of compound $\mathbf{1 y}$

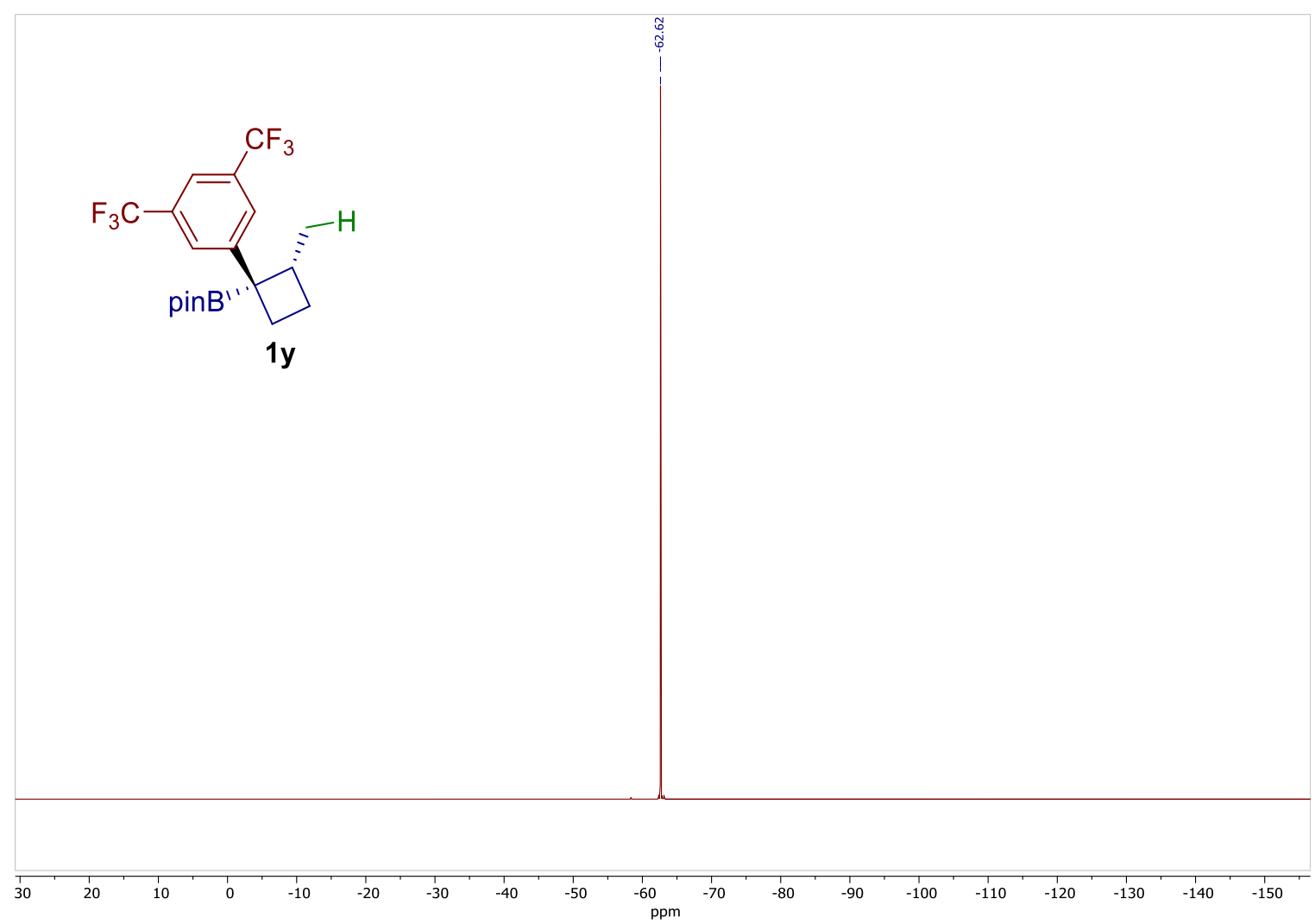


${ }^{\mathbf{1}} \mathbf{H}-\mathbf{N M R}\left(400 \mathrm{MHz}, \mathrm{CDCl}_{3}\right)$ of compound $\mathbf{1 z}$ see procedure

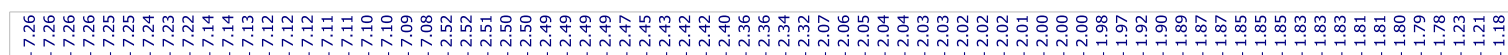

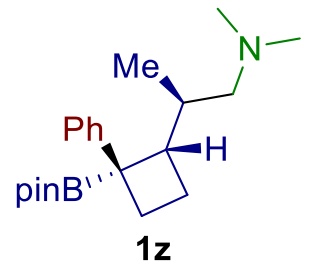
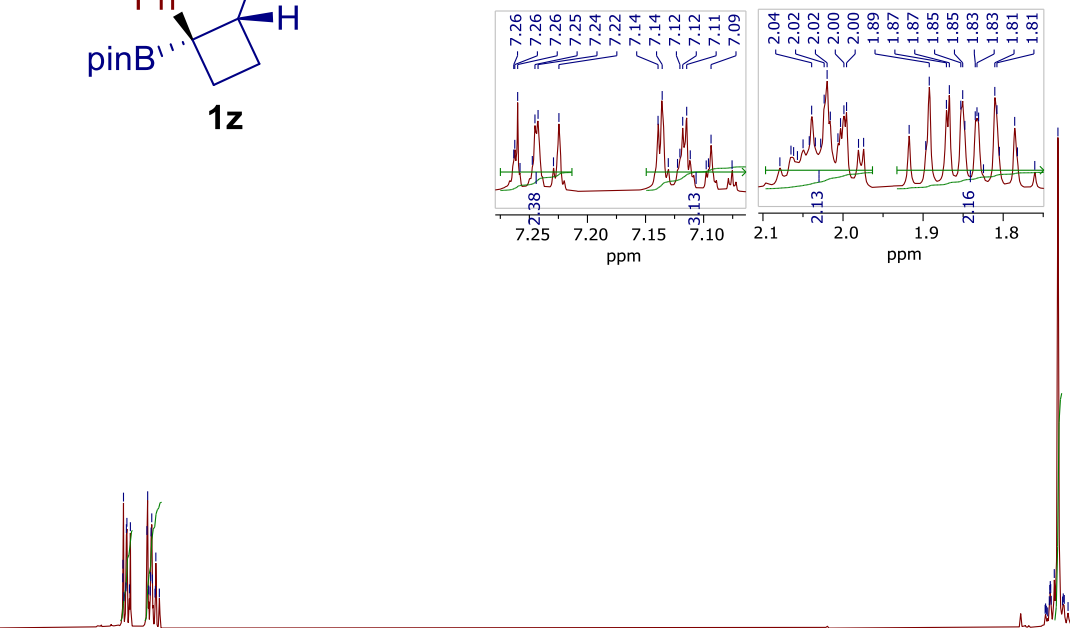

T'

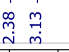

T'T $ヤ \longmapsto$

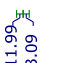

$\begin{array}{llll}6.0 & 5.5 & 5.0 & 4.5\end{array}$

${ }^{13} \mathbf{C}-\mathbf{N M R}\left(100 \mathrm{MHz}, \mathrm{CDCl}_{3}\right)$ of compound $\mathbf{1 z}$

\begin{tabular}{|c|}
\hline 37 \\
\hline
\end{tabular}

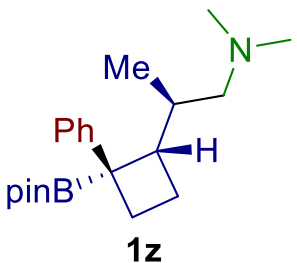

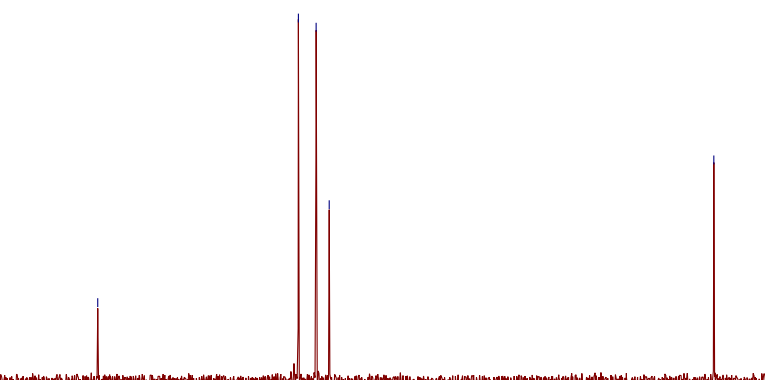

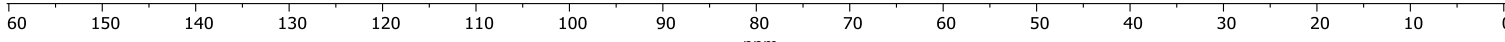


${ }^{1} \mathbf{H}-\mathbf{N M R}\left(400 \mathrm{MHz}, \mathrm{CDCl}_{3}\right)$ of compound 1 aa see procedure

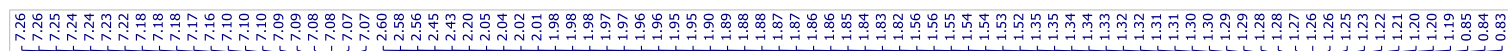

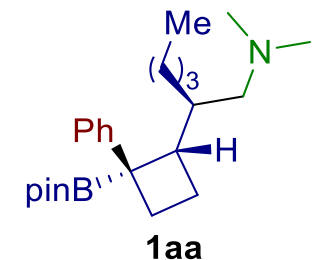
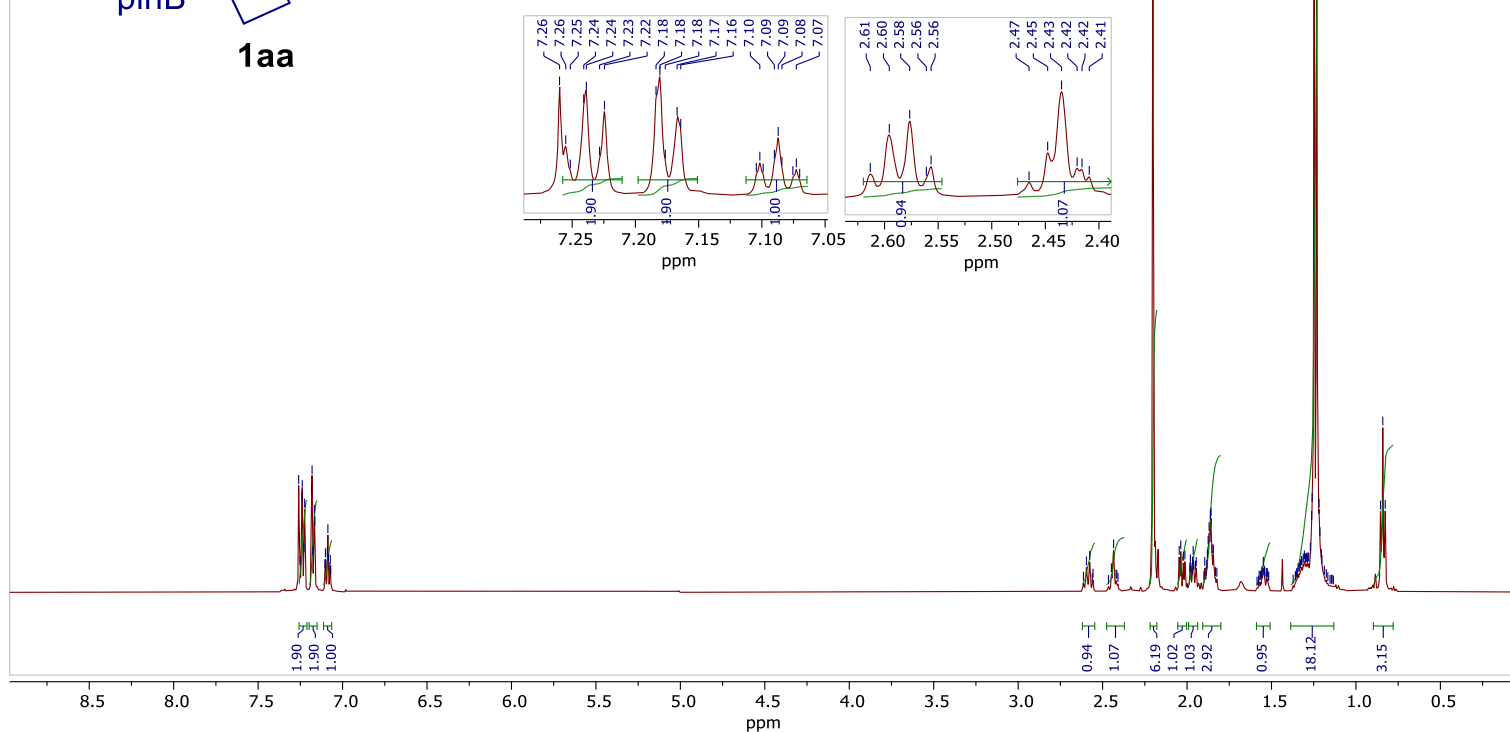

${ }^{13}$ C-NMR (100 MHz, $\left.\mathrm{CDCl}_{3}\right)$ of compound $\mathbf{1 a a}$

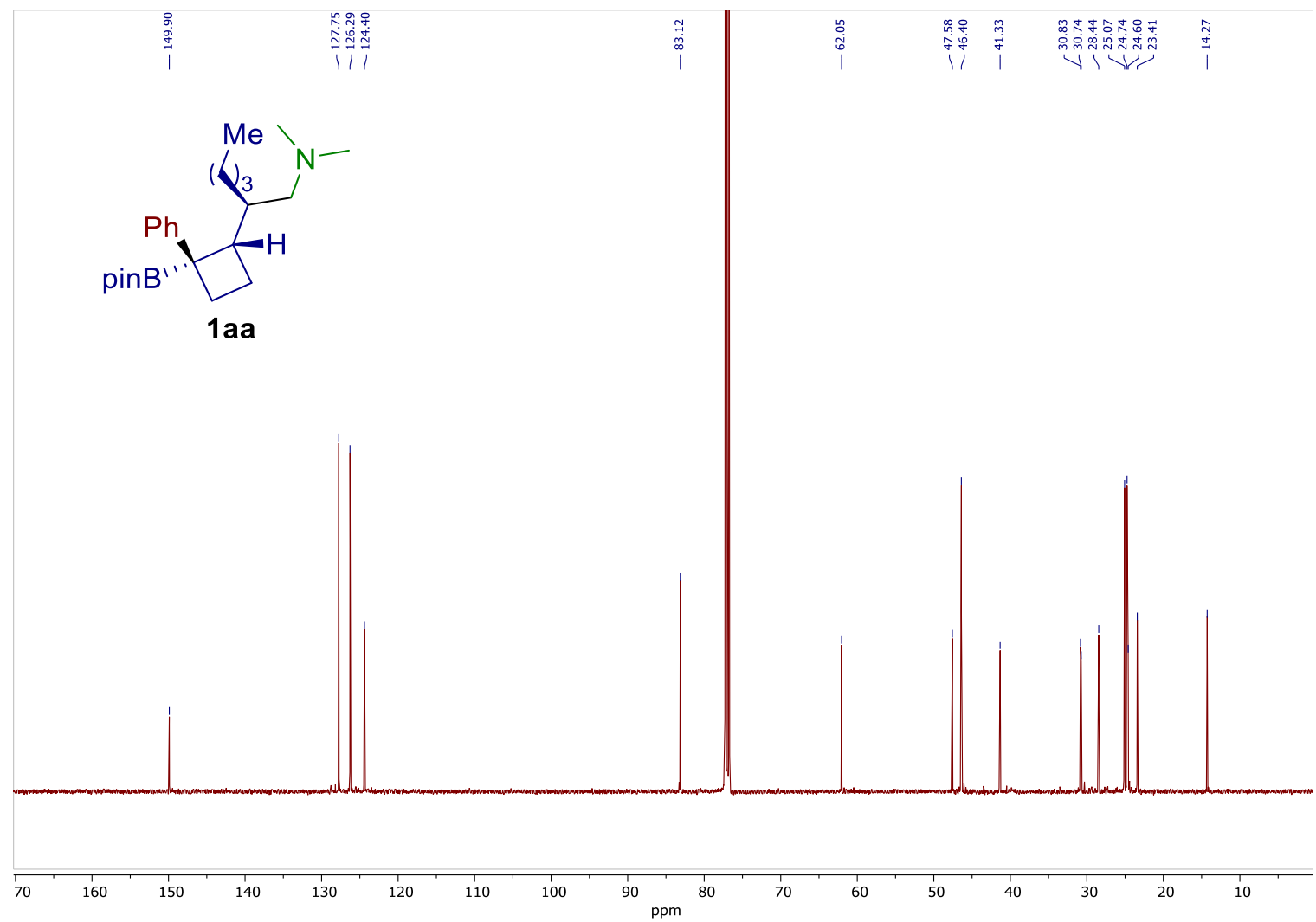


${ }^{1} \mathbf{H}-\mathbf{N M R}\left(400 \mathrm{MHz}, \mathrm{CDCl}_{3}\right)$ of compound $\mathbf{1 a b}$ see procedure

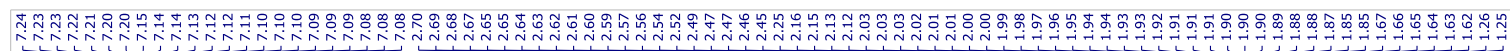

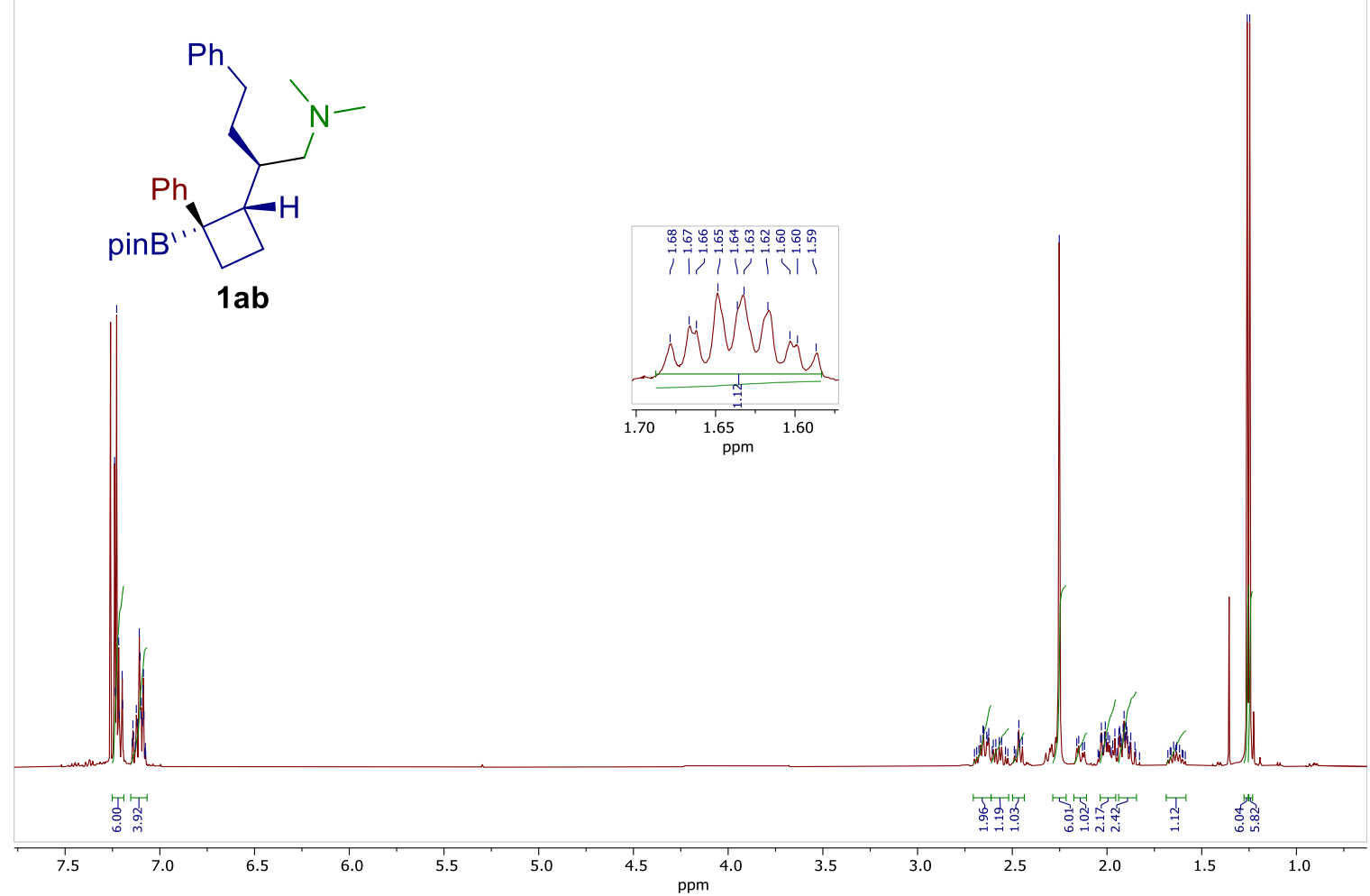

${ }^{13} \mathbf{C}-\mathbf{N M R}\left(100 \mathrm{MHz}, \mathrm{CDCl}_{3}\right)$ of compound 1ab

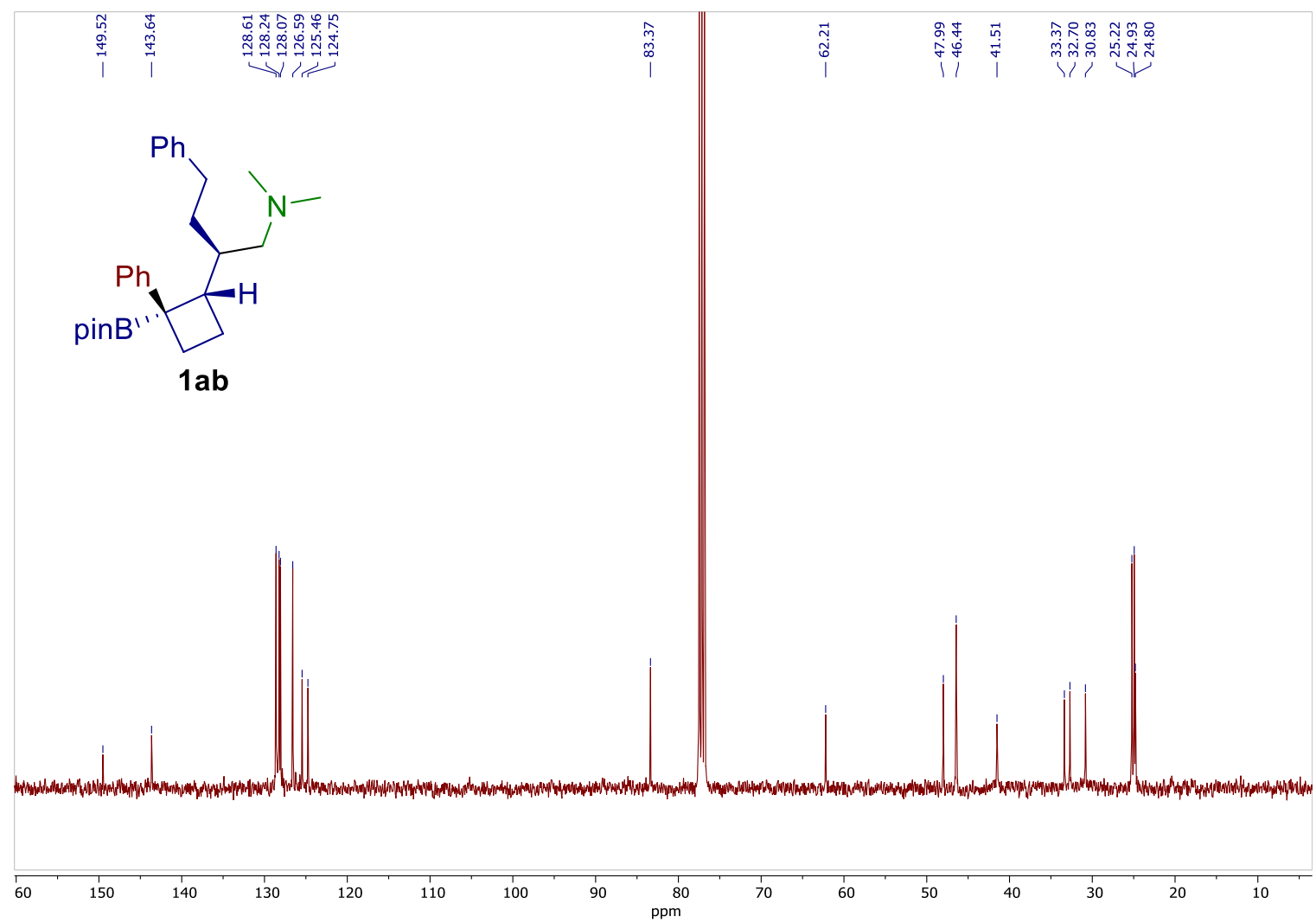


${ }^{1} \mathbf{H}-\mathbf{N M R}\left(400 \mathrm{MHz}, \mathrm{CDCl}_{3}\right)$ of compound $1 \mathbf{a c}$ see procedure

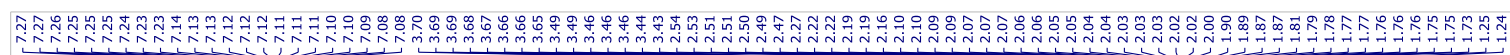<smiles>CN(C)CC(CCO)[C@H]1C[C@@H]2[C@H]([18OH])[C@H]1[C@H]2c1ccccc1</smiles>
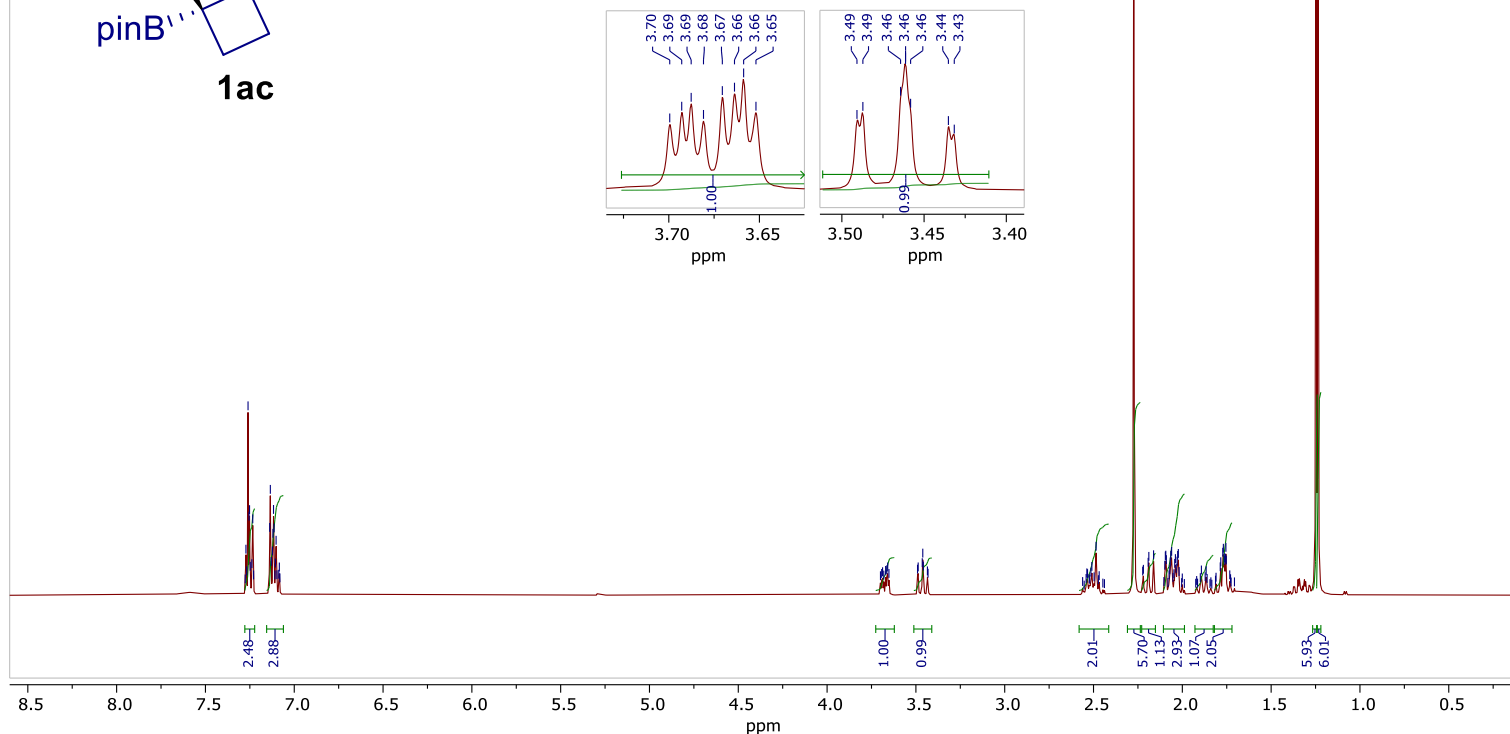

${ }^{13} \mathbf{C}-\mathbf{N M R}\left(100 \mathrm{MHz}, \mathrm{CDCl}_{3}\right)$ of compound $\mathbf{1 a c}$

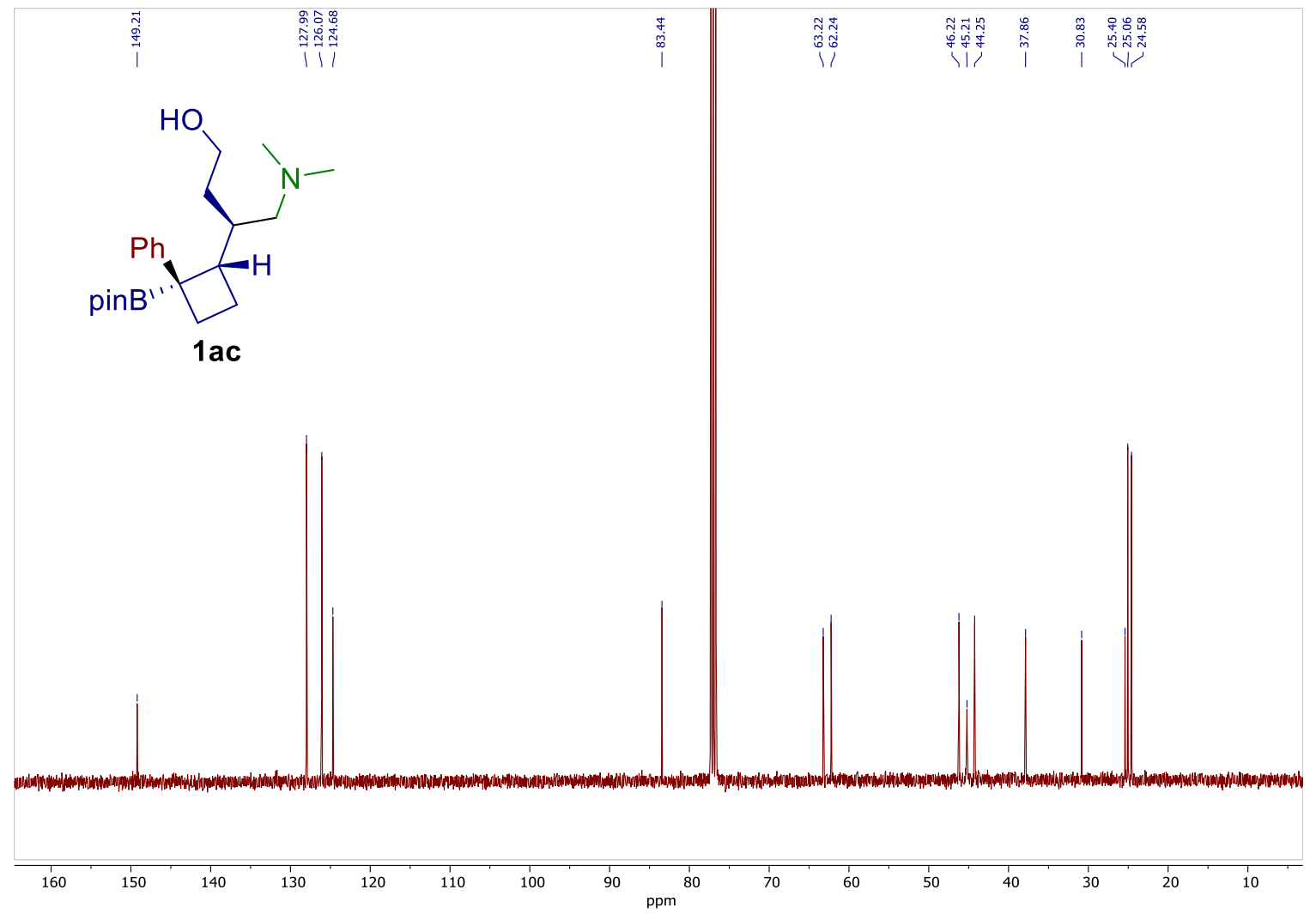


${ }^{1} \mathbf{H}-\mathbf{N M R}\left(400 \mathrm{MHz}, \mathrm{CDCl}_{3}\right)$ of compound $\mathbf{1 a d}$ see procedure

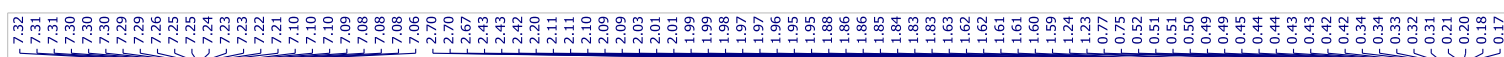

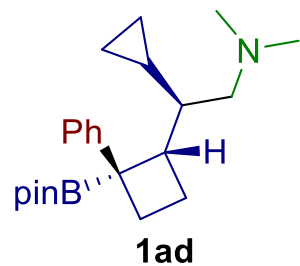
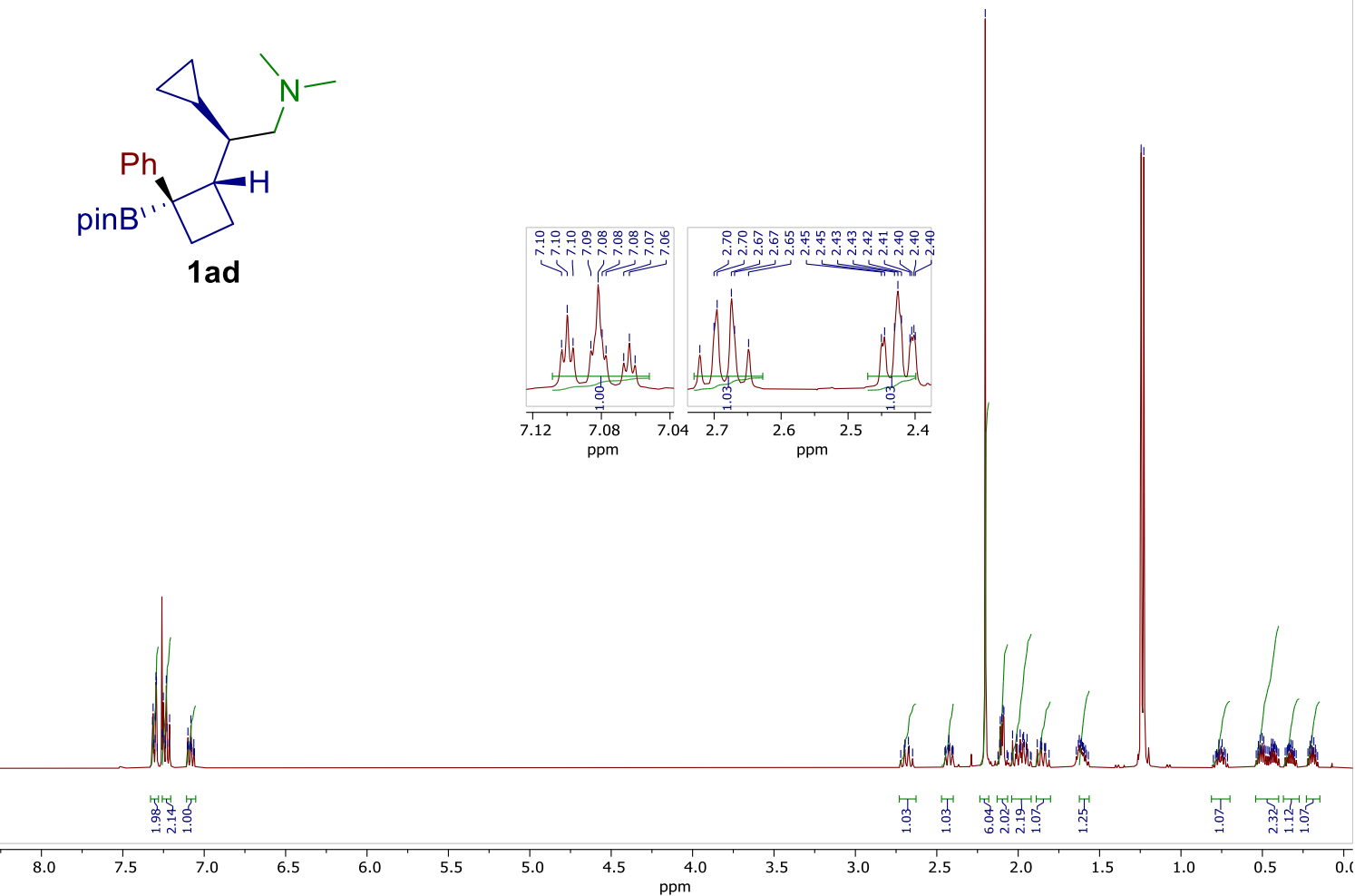

${ }^{13} \mathbf{C}-\mathbf{N M R}\left(100 \mathrm{MHz}, \mathrm{CDCl}_{3}\right)$ of compound 1ad

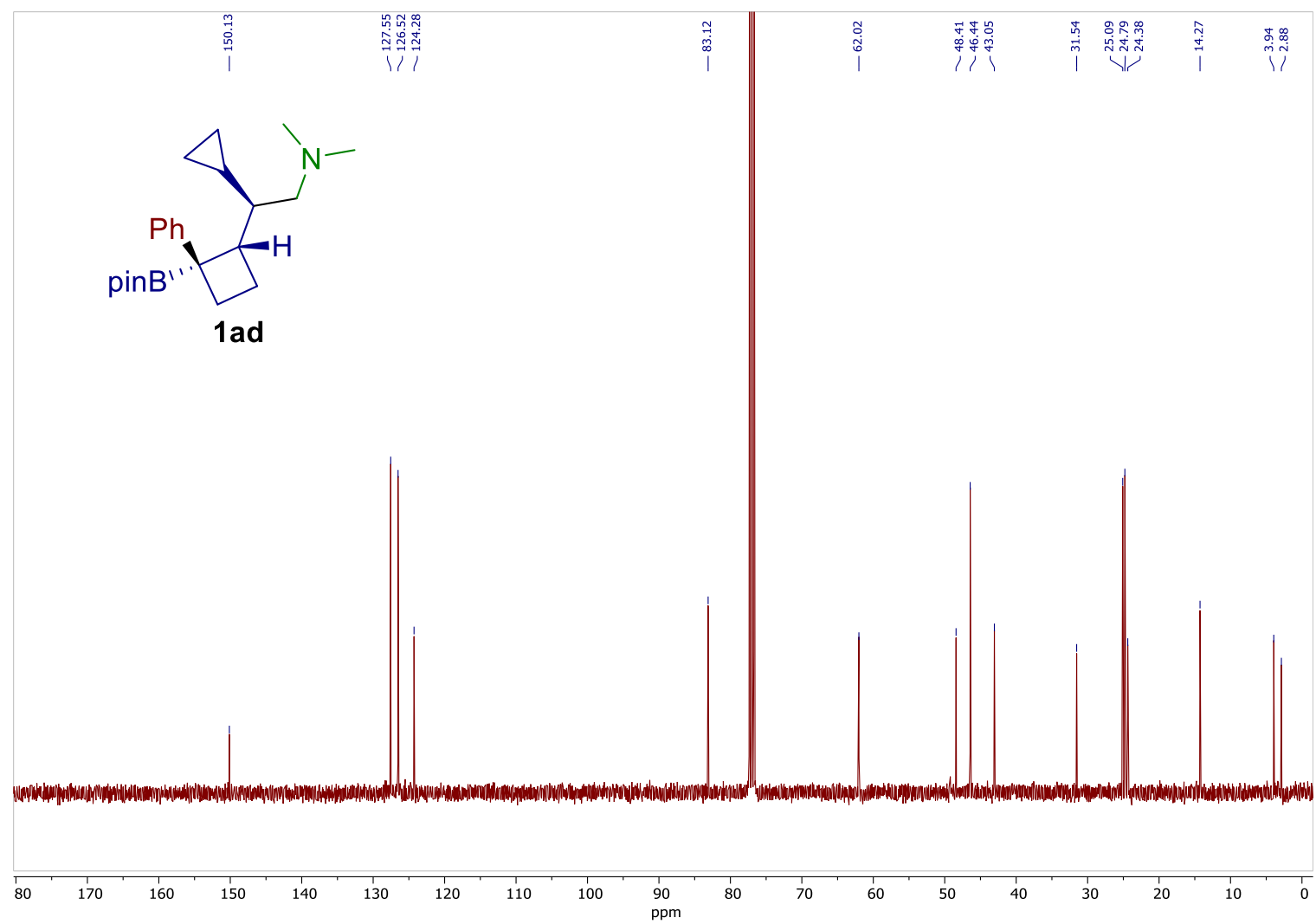


${ }^{1} \mathbf{H}-\mathbf{N M R}\left(400 \mathrm{MHz}, \mathrm{CDCl}_{3}\right)$ of compound $\mathbf{1 a e}$ see procedure

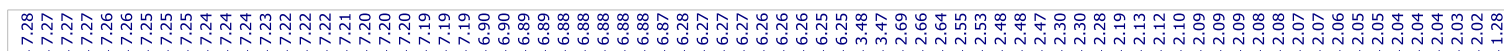<smiles>CN(C)C[C@H](c1ccccc1)[C@H]1CC[C@@H]1[PbH]</smiles>

1 ae
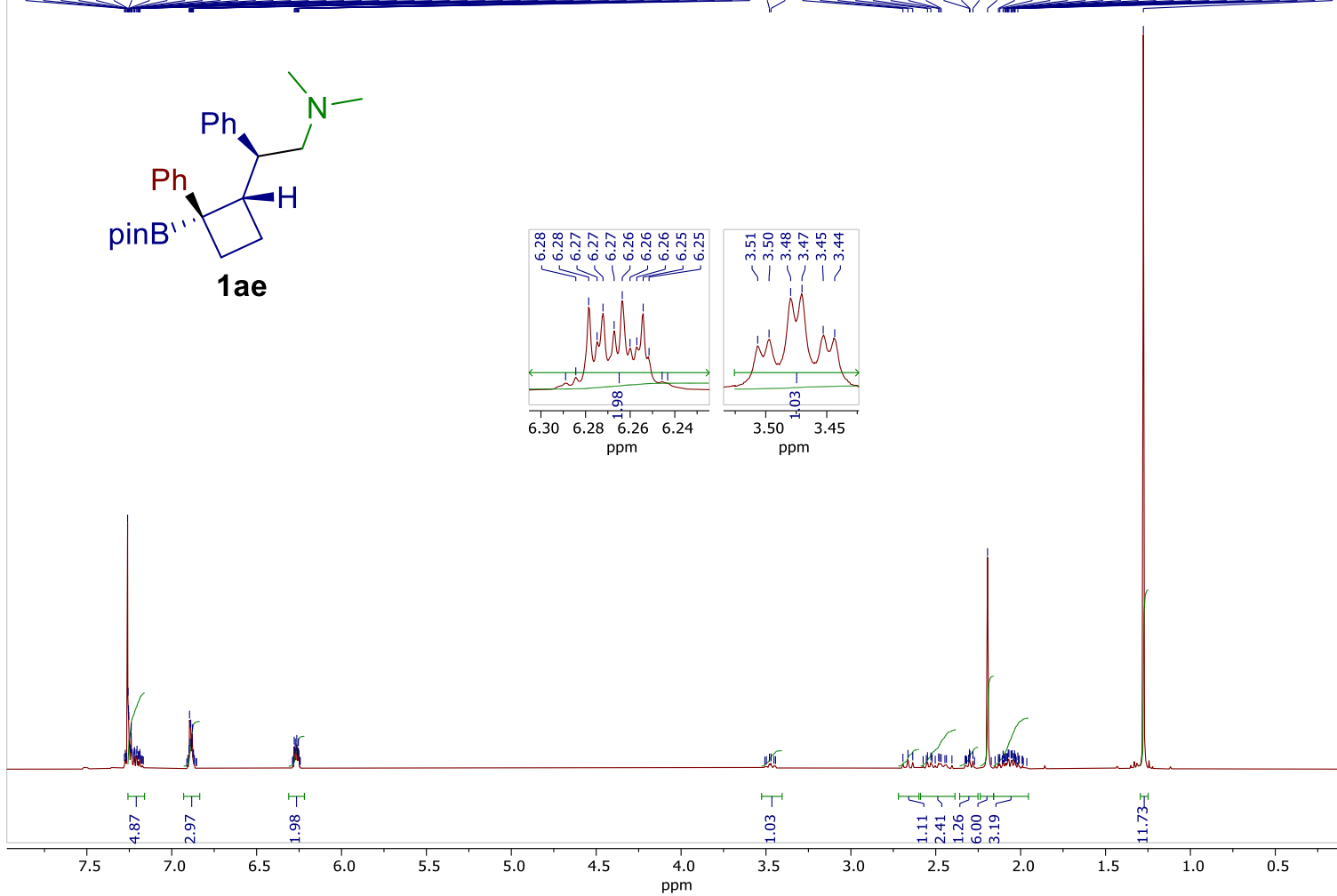

${ }^{13} \mathbf{C}-\mathbf{N M R}\left(100 \mathrm{MHz}, \mathrm{CDCl}_{3}\right)$ of compound 1ae

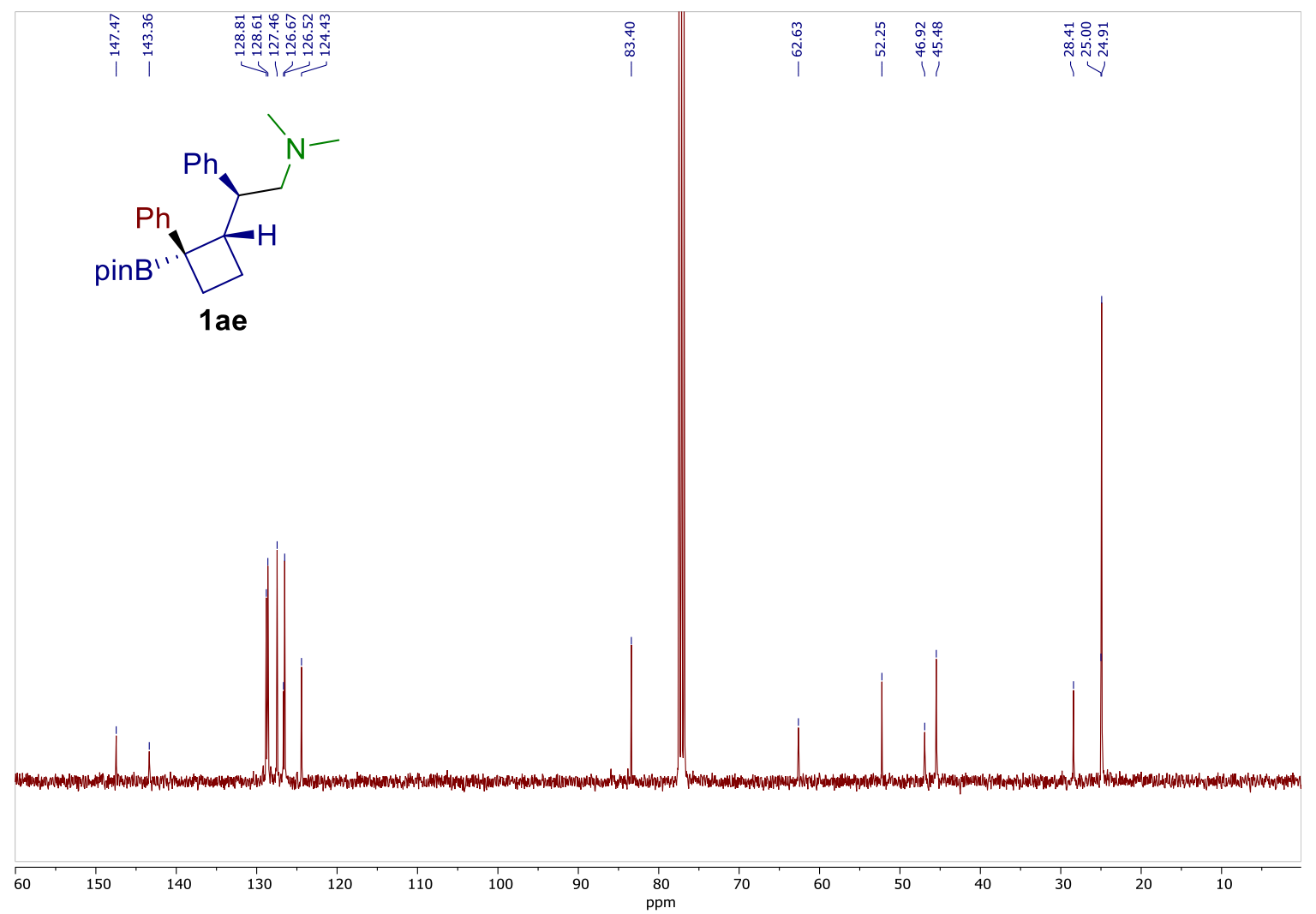


${ }^{1} \mathbf{H}-\mathbf{N M R}\left(400 \mathrm{MHz}, \mathrm{CDCl}_{3}\right)$ of compound $\mathbf{1 a f}$ see procedure

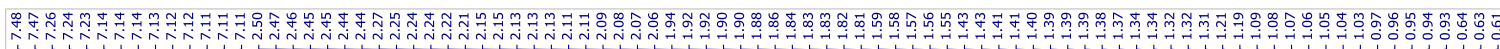

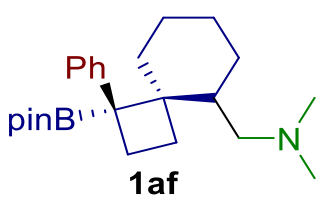
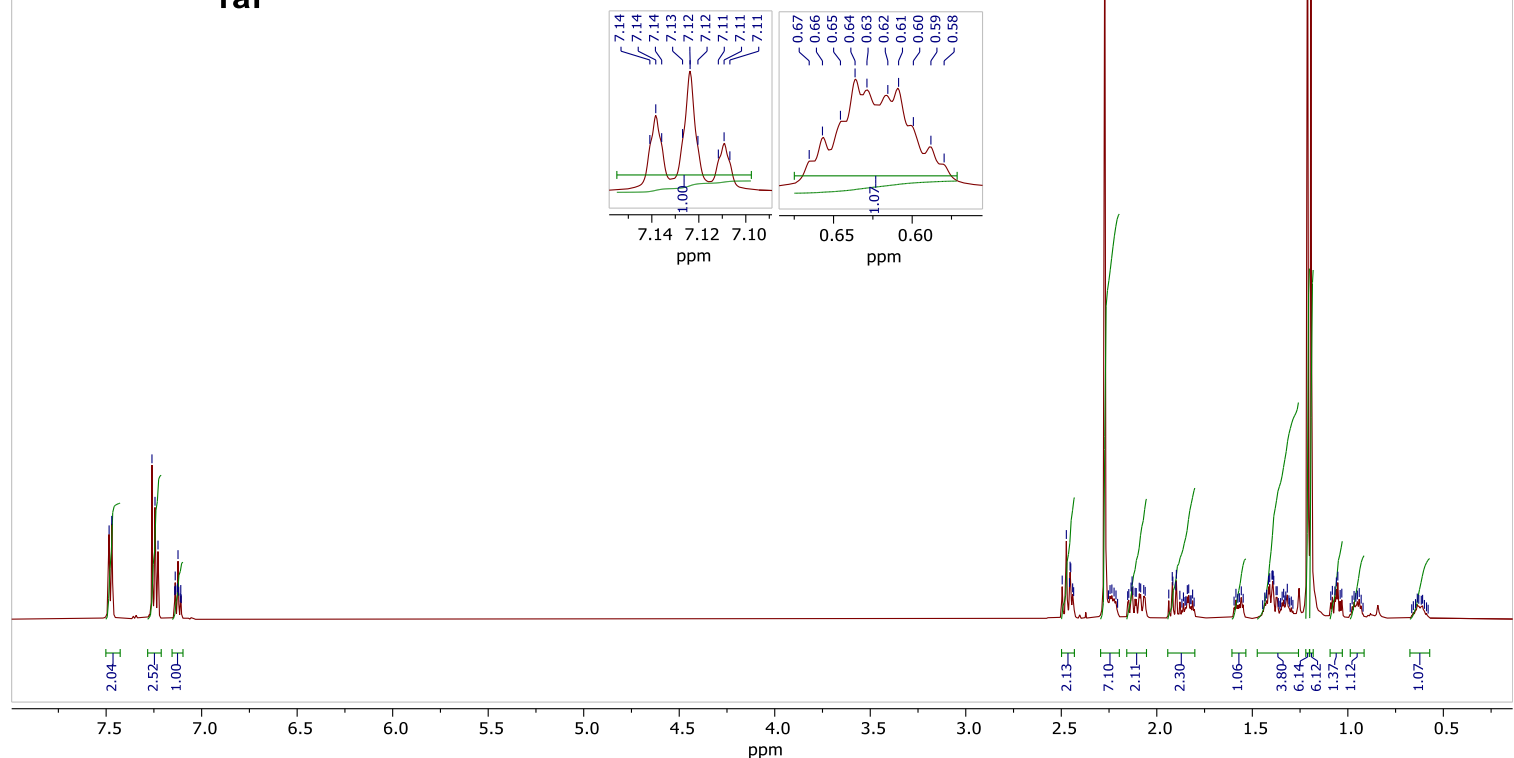

${ }^{13}$ C-NMR $\left(100 \mathrm{MHz}, \mathrm{CDCl}_{3}\right)$ of compound $\mathbf{1 a f}$

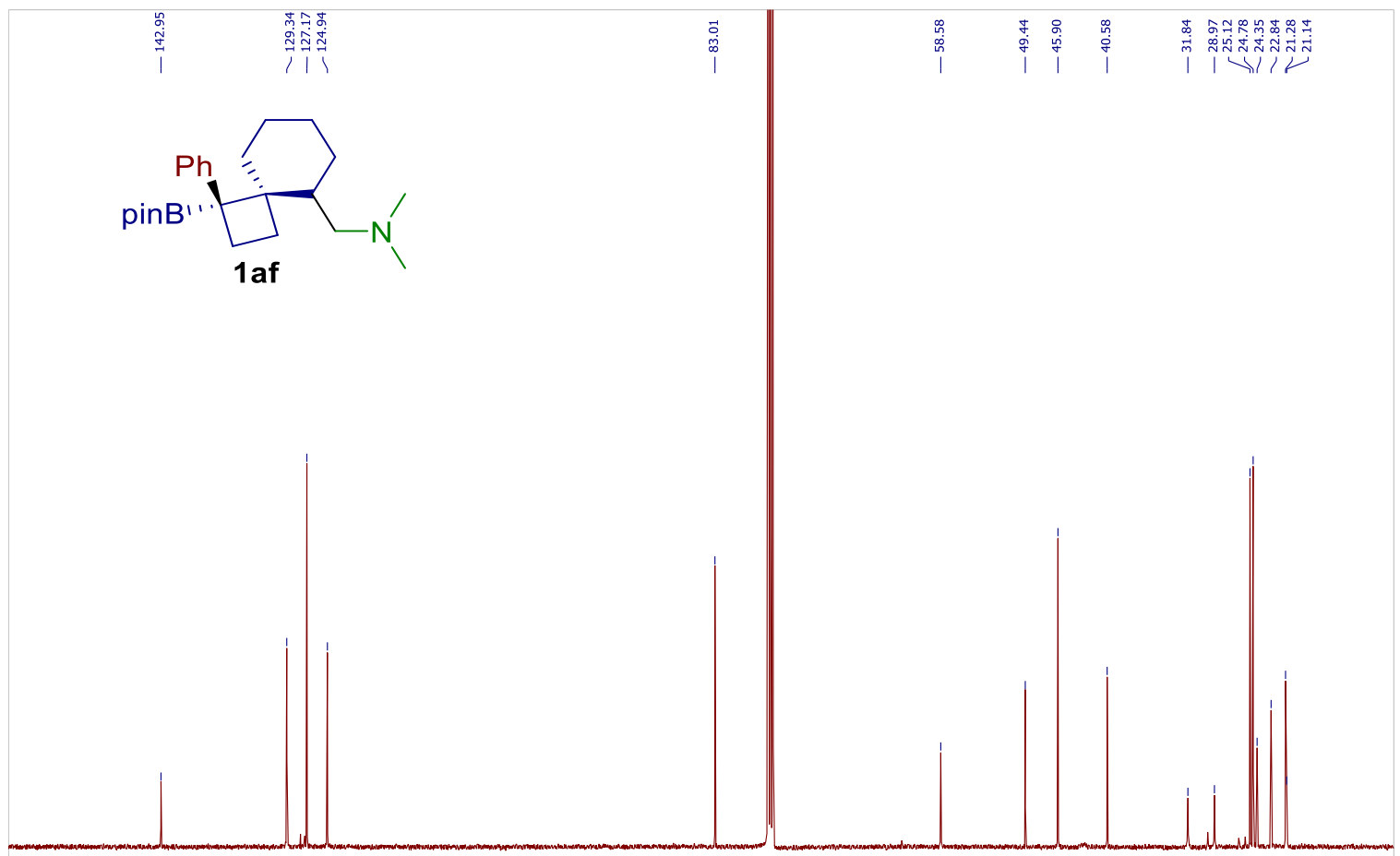

$\begin{array}{lllllllllllllllllllllllllllllllllllllllllllllllll}155 & 150 & 145 & 140 & 135 & 130 & 125 & 120 & 115 & 110 & 105 & 100 & 95 & 90 & 85 & 80 & 75 & 70 & 65 & 60 & 55 & 50 & 45 & 40 & 35 & 30 & 25 & 20 & 15 & 1\end{array}$ 
${ }^{\mathbf{1}} \mathbf{H}-\mathbf{N M R}\left(400 \mathrm{MHz}, \mathrm{CDCl}_{3}\right)$ of compound $\mathbf{2 b}$ see procedure
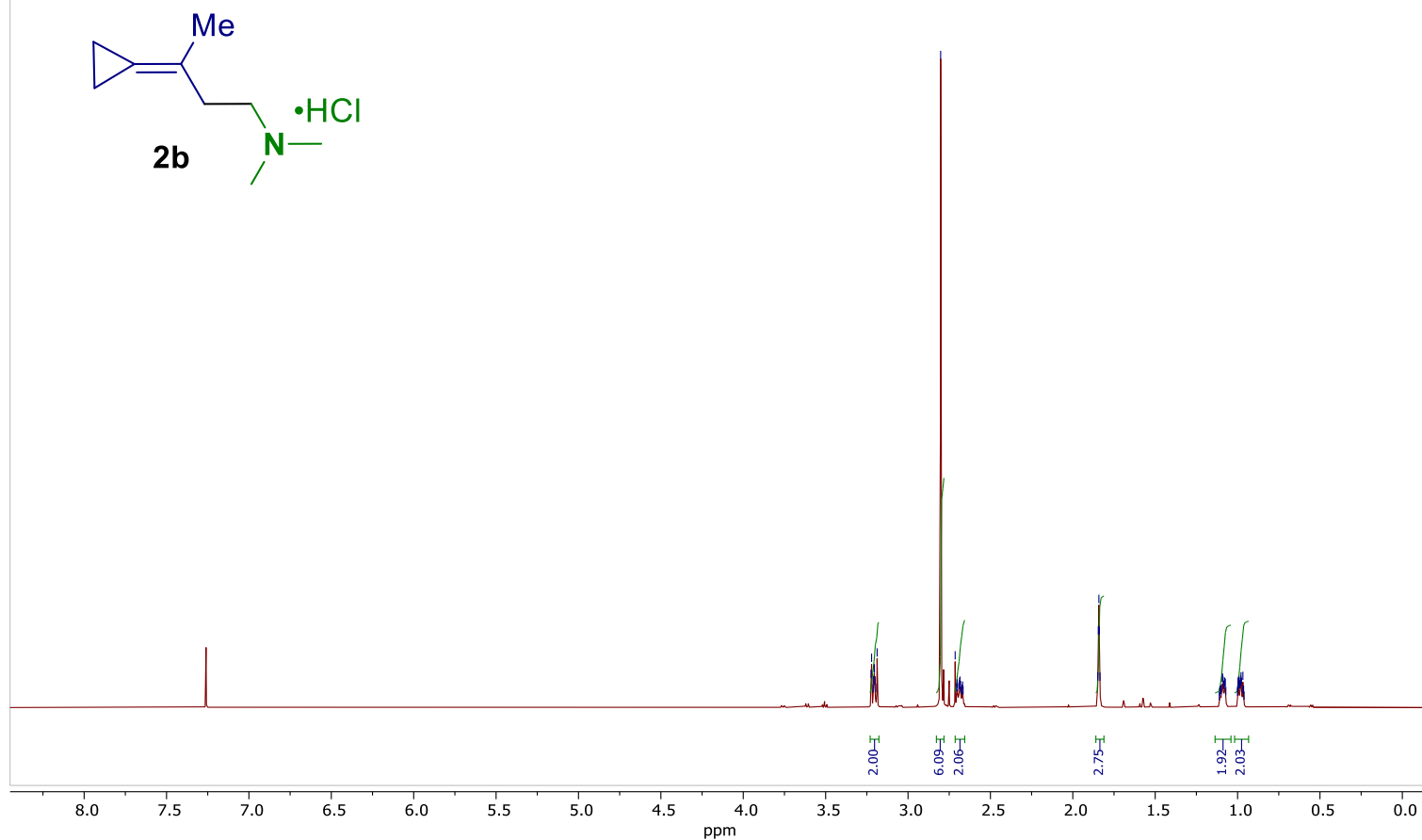

${ }^{13} \mathbf{C}-\mathbf{N M R}\left(100 \mathrm{MHz}, \mathrm{CDCl}_{3}\right)$ of compound $\mathbf{2 b}$

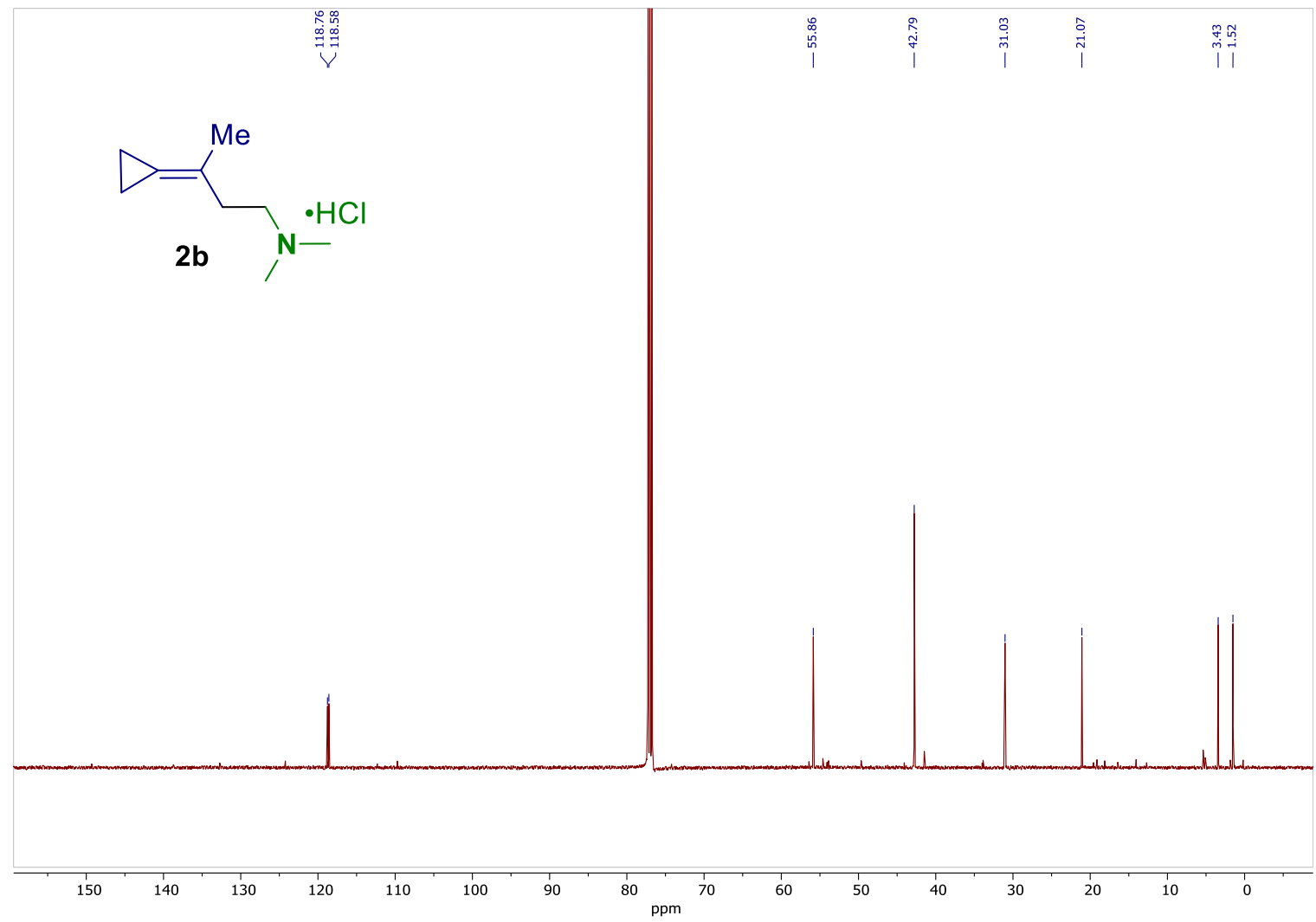


${ }^{1} \mathbf{H}-\mathbf{N M R}\left(400 \mathrm{MHz}, \mathrm{CDCl}_{3}\right)$ of compound $\mathbf{1 a g}$ see procedure

\section{gog}
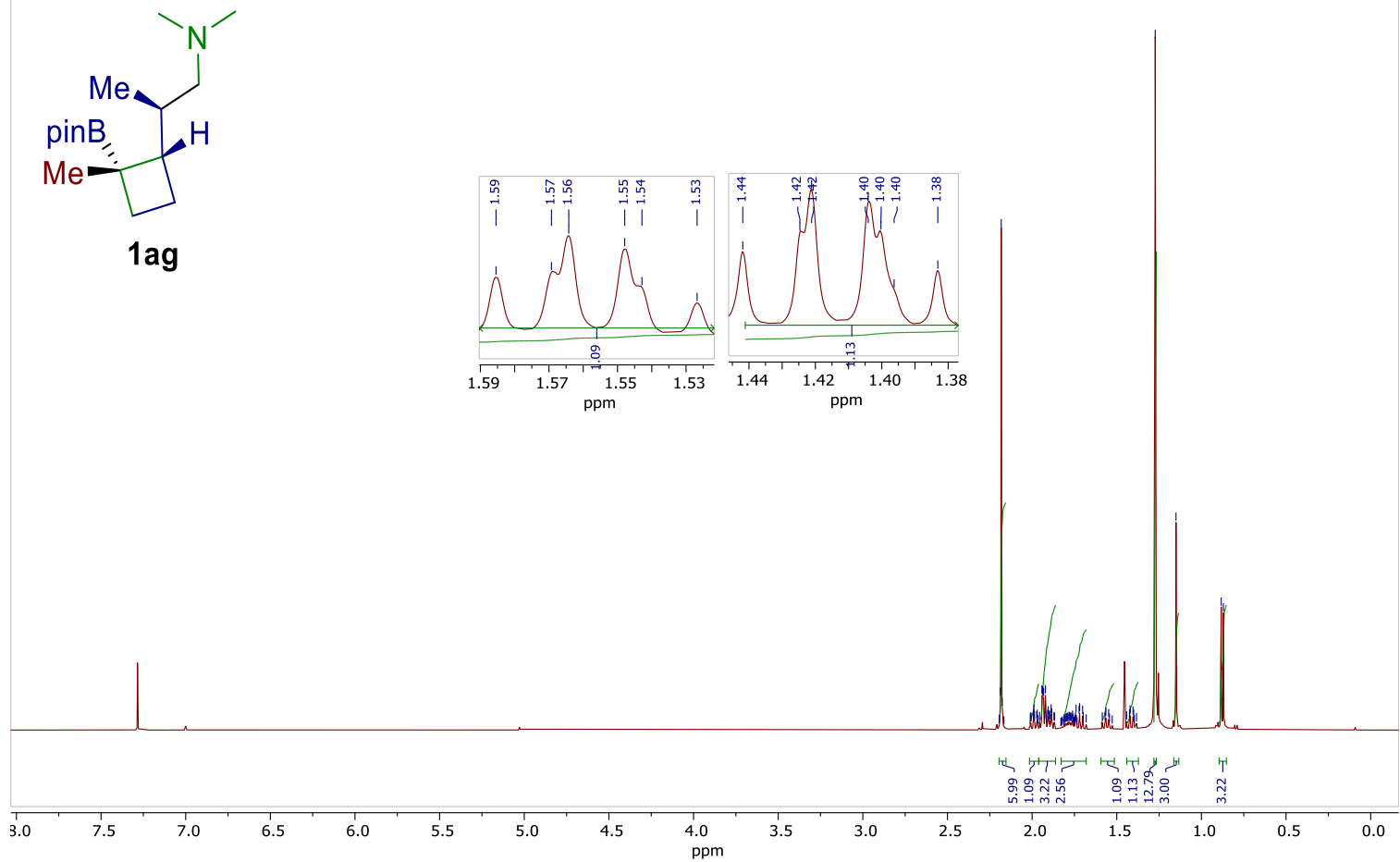

${ }^{13}$ C-NMR (100 MHz, $\left.\mathrm{CDCl}_{3}\right)$ of compound $\mathbf{1 a g}$

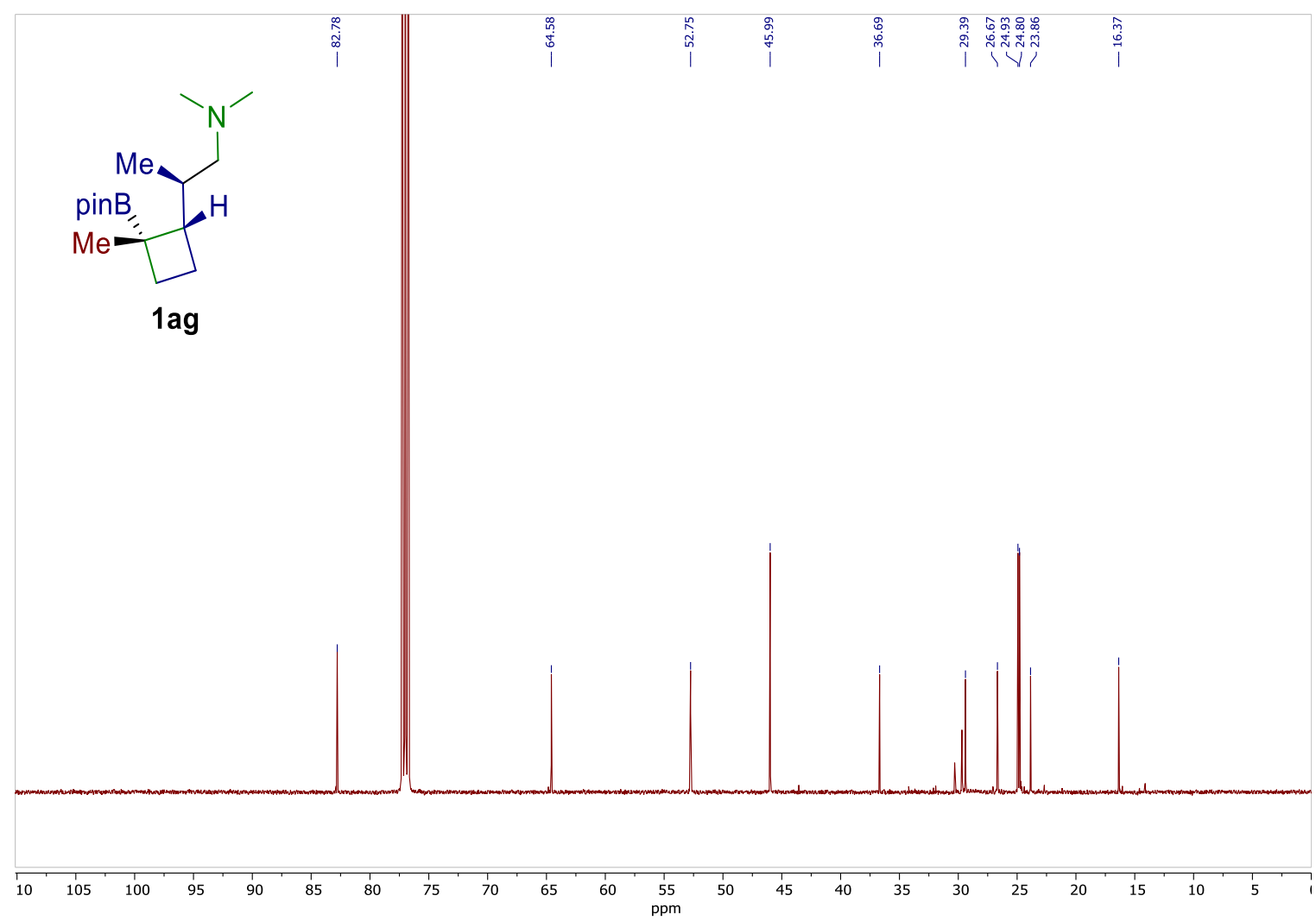


${ }^{1} \mathbf{H}-\mathbf{N M R}\left(400 \mathrm{MHz}, \mathrm{CDCl}_{3}\right)$ of compound $\mathbf{6}$ see procedure

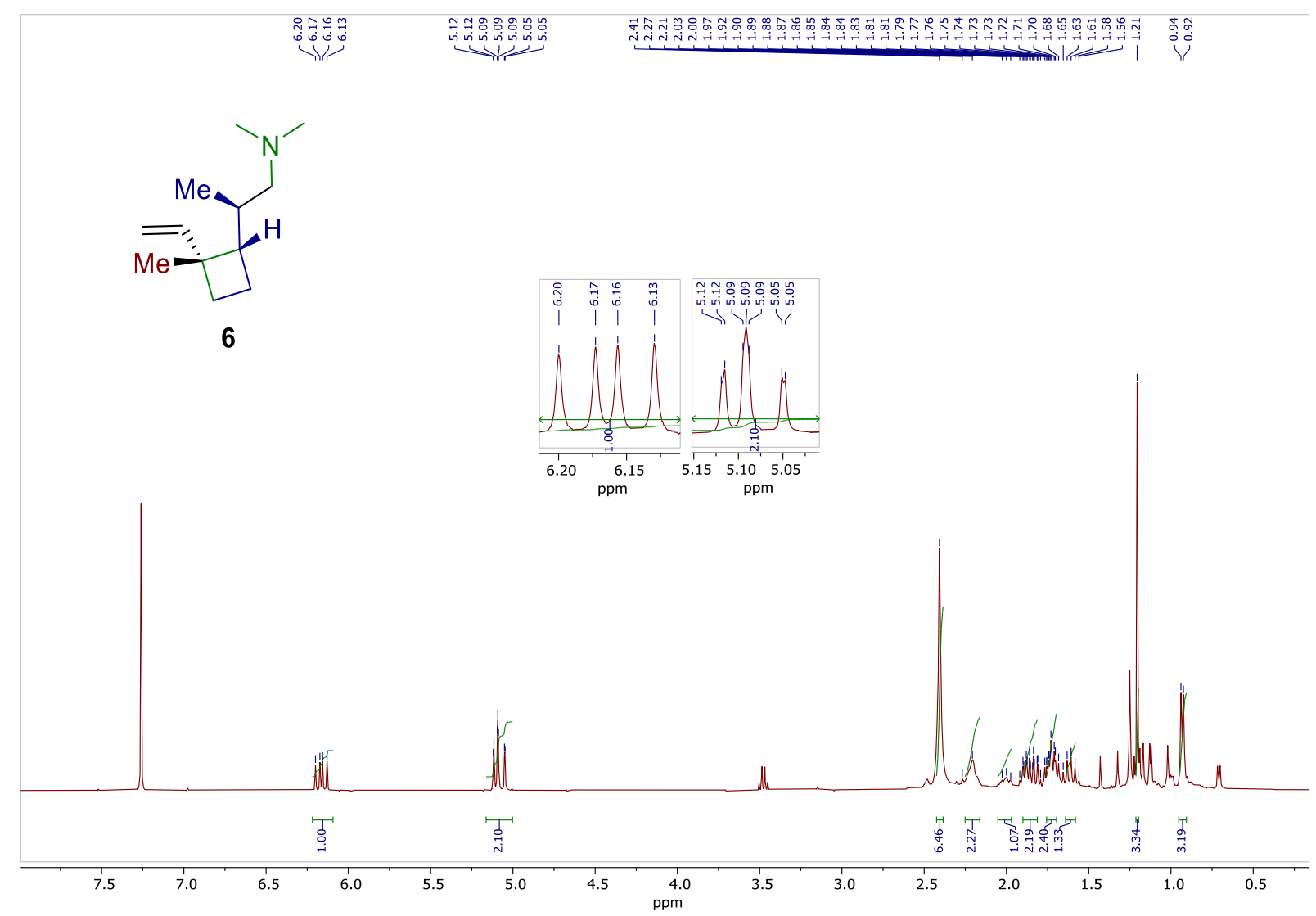


${ }^{1} \mathbf{H}-\mathbf{N M R}\left(400 \mathrm{MHz}, \mathrm{CDCl}_{3}\right)$ of compound $\mathbf{7}$ see procedure

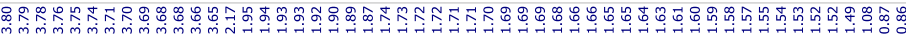
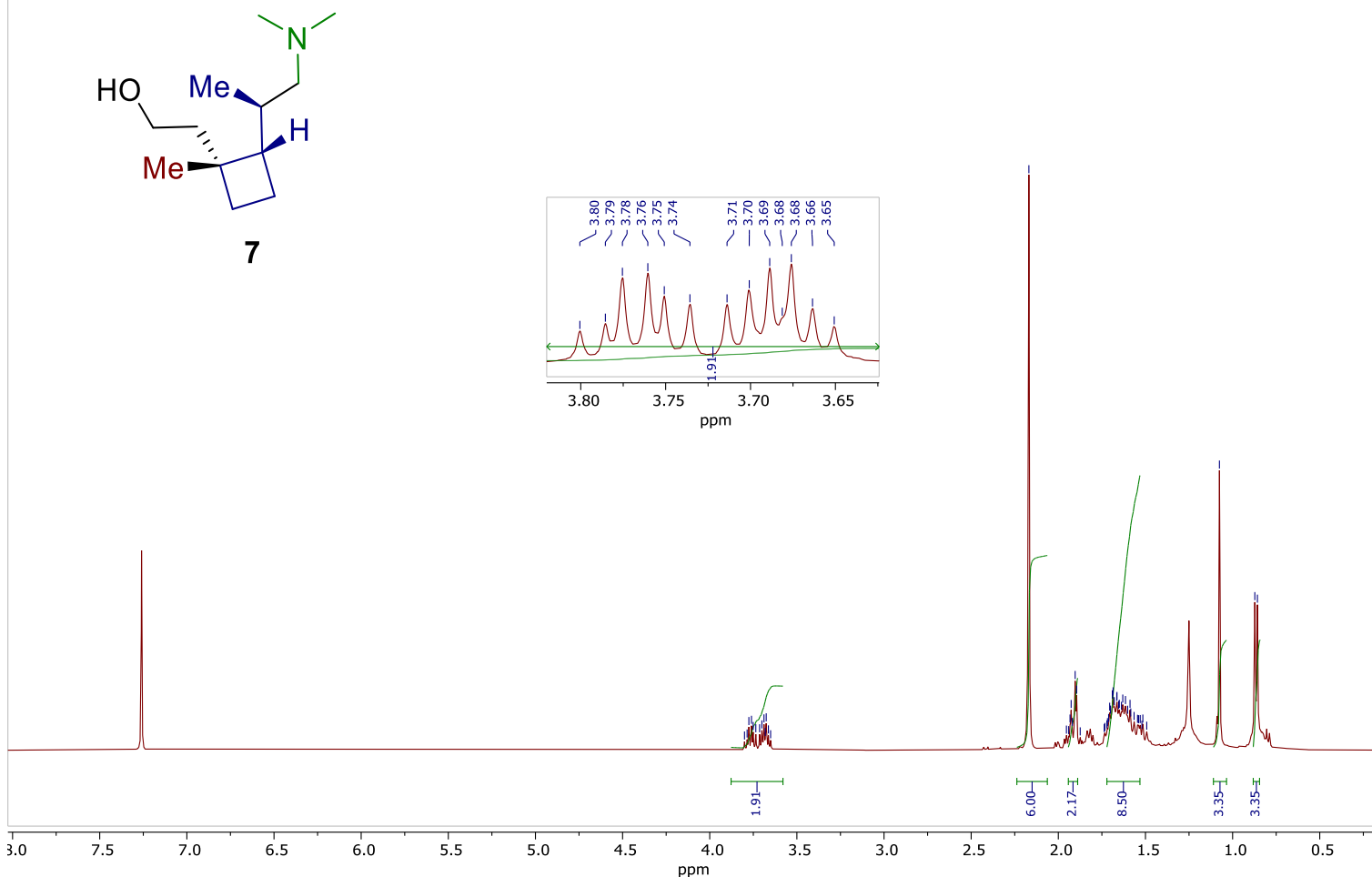

${ }^{13}$ C-NMR (100 MHz, $\left.\mathrm{CDCl}_{3}\right)$ of compound $\mathbf{1 a g}$

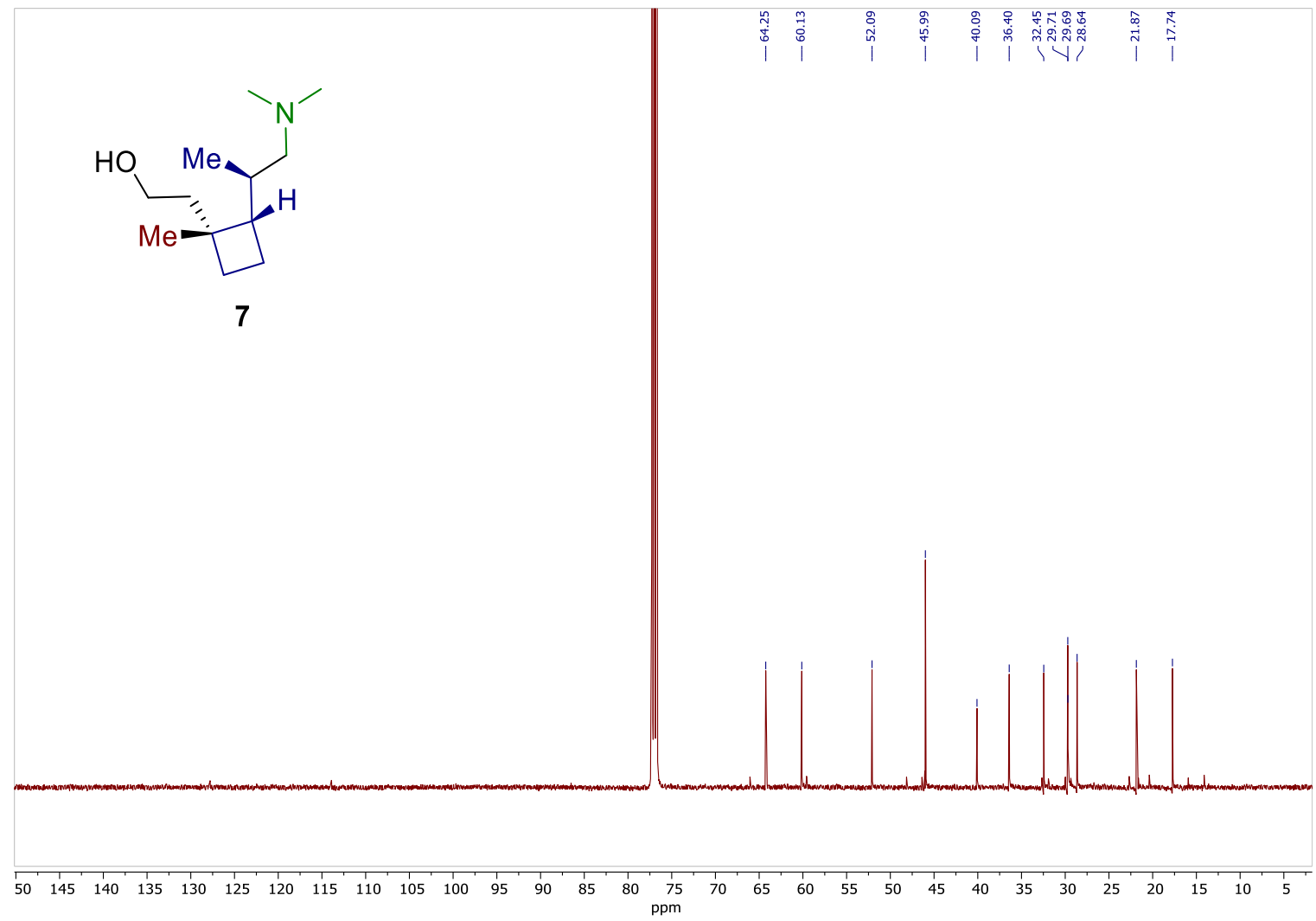


${ }^{1} \mathbf{H}-\mathbf{N M R}\left(400 \mathrm{MHz}, \mathrm{CDCl}_{3}\right)$ of compound $( \pm)$-Grandisol see procedure

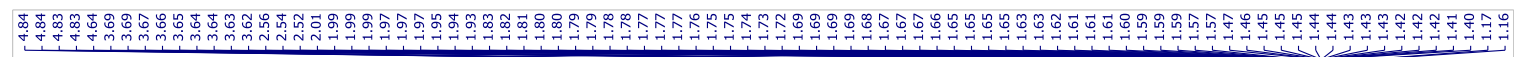<smiles>C=C(C)[C@H]1CC[C@@]1(C)CCO</smiles>

$( \pm)$-Grandisol
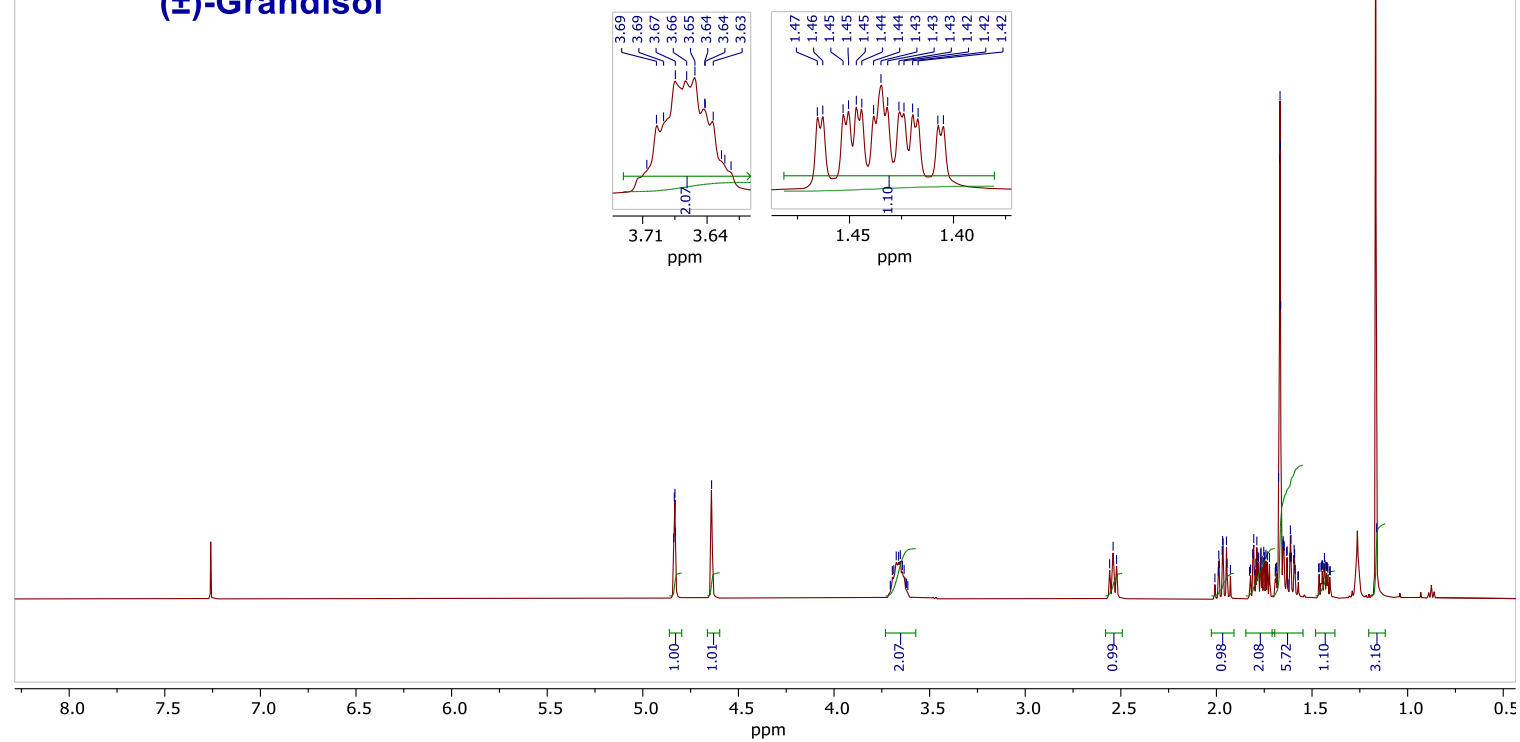

${ }^{13} \mathbf{C}-\mathbf{N M R}\left(100 \mathrm{MHz}, \mathrm{CDCl}_{3}\right)$ of compound ( \pm )-Grandisol

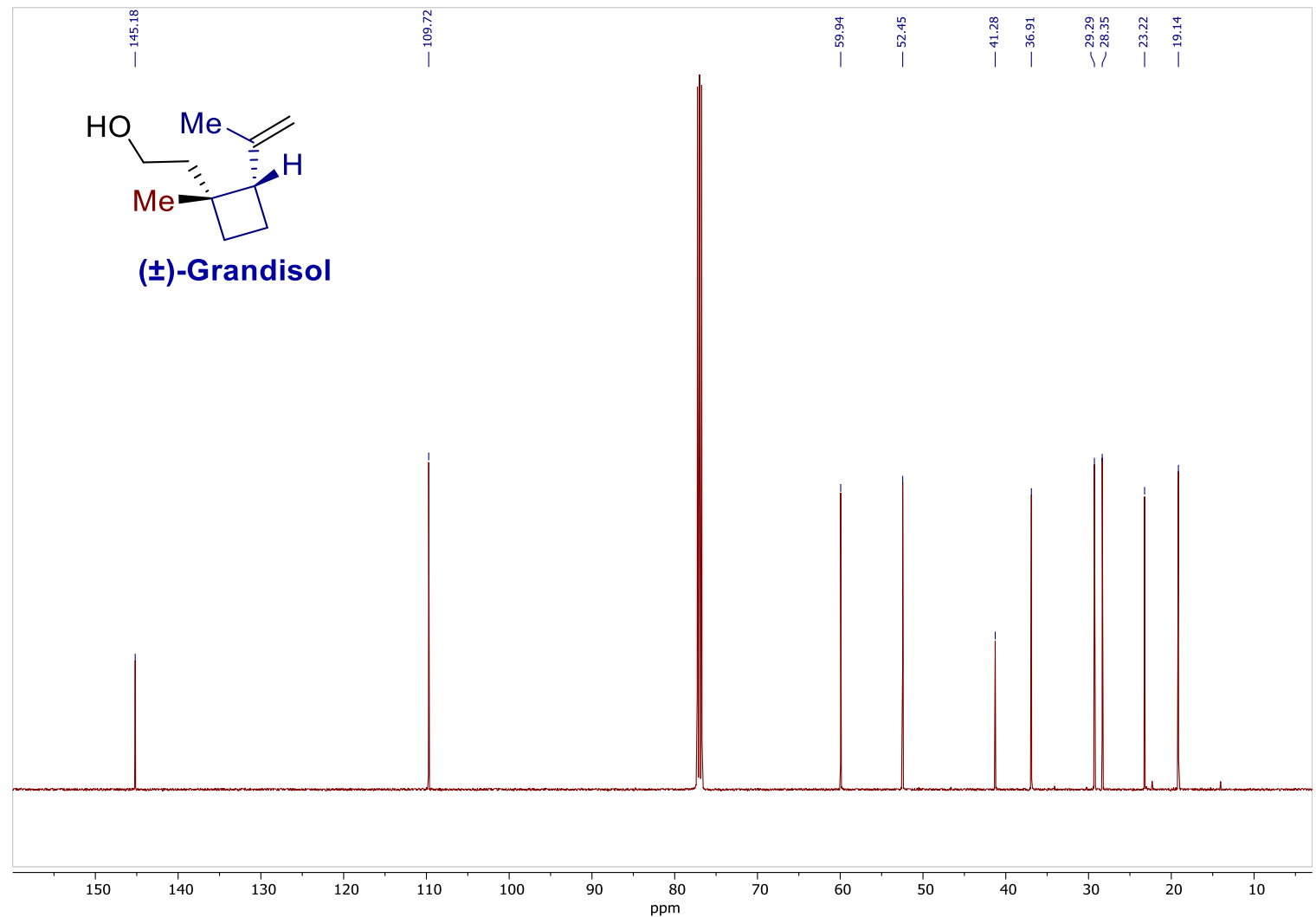




\section{Crystal structure and structural assignments of the products}

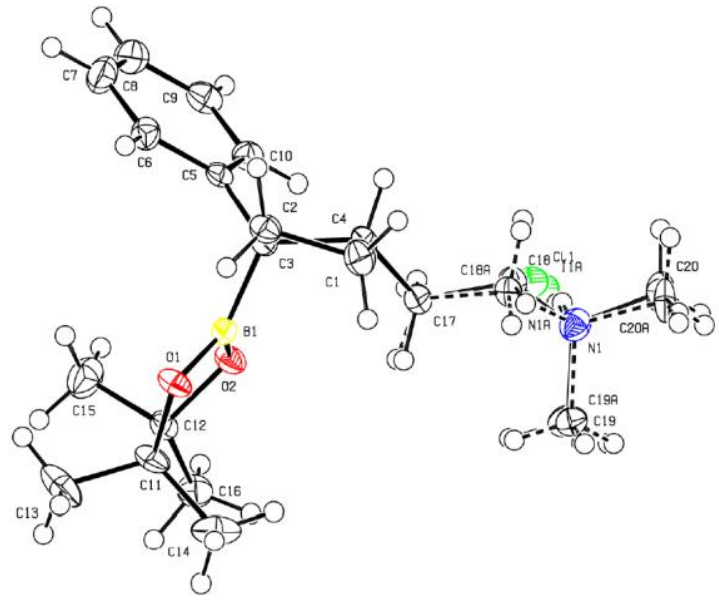

X-ray of 1a, CCDC 1976972

\section{Procedure for 8 (Mattson homologation):}

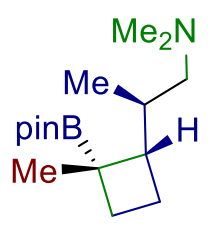

$1 \mathrm{ag}$

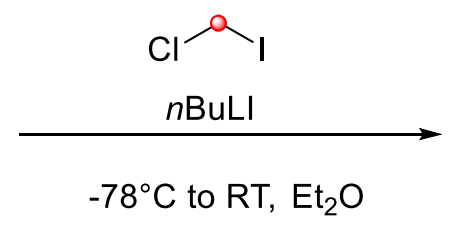

$-78^{\circ} \mathrm{C}$ to $\mathrm{RT}, \mathrm{Et}_{2} \mathrm{O}$

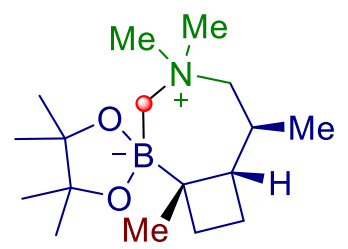

8

The boronic ester $1 \mathrm{ag}(28.1 \mathrm{mg}, 0.100 \mathrm{mmol}, 1.00$ equiv) and iodochloromethane $(21.9 \mu \mathrm{L}$, $0.300 \mathrm{mmol}, 3.00$ equiv) were dissolved in diethyl ether $(1 \mathrm{~mL})$ and cooled to $-95{ }^{\circ} \mathrm{C}$ (methanol/liquid nitrogen bath). ${ }^{n} \mathrm{BuLi}(0.295 \mathrm{mmol}, 1.60 \mathrm{M}$ solution in hexane, 0.295 equiv) was added dropwise and the solution was stirred for 10 min at $-95^{\circ} \mathrm{C}$, followed by additional $1 \mathrm{~h}$ at room temperature. The whole mixture was plugged through a thin layer of silica and eluted with $\mathrm{Et}_{2} \mathrm{O}$. Solvents were evaporated to afford the crude boronic ester. The crude mixture was purified by column chromatography using pentane:dichloromethane 95:5 - 85:15 to afford the ate complex as a white solid in $95 \%$ yield $(23 \mathrm{mg})$. The compound $\mathbf{8}$ was recrystallized in pentane/diethyl ether. 


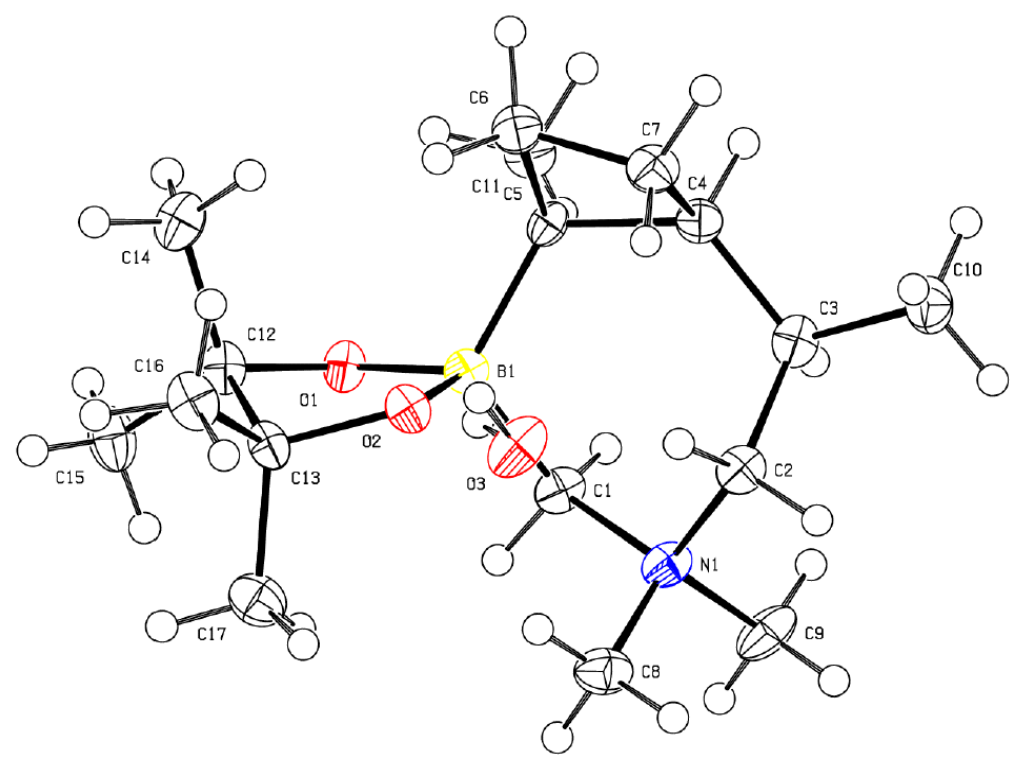

X-ray of 8, CCDC 1985237

X-ray diffraction experiments on $\mathbf{1 a}$ and $\mathbf{8}$ were carried out at 100(2) K on a Bruker APEX II CCD diffractometer using Mo-K $\mathrm{K}_{\alpha}$ radiation $(\lambda=0.71073 \AA)$. Intensities were integrated in SAINT $^{6}$ and absorption corrections based on equivalent reflections were applied using SADABS. ${ }^{7}$ The structure were both solved using ShelXT ${ }^{8}$ and refined by full matrix least squares against $F^{7}$ in ShelXL ${ }^{9}$ using Olex $2^{10}$. All of the non-hydrogen atoms were refined anisotropically. While all of the hydrogen atoms were located geometrically and refined using a riding model. In the case of $\mathbf{1 a}$ the molecule displayed disorder, the counterion was found to be a mixture of $\mathrm{Cl}^{-}$and $\mathrm{I}^{-}$both of which were present in the solution from which the crystals were obtained. The counterion disorder results in disorder in the main chain due to the presence of $\mathrm{N}-\mathrm{H} \cdots$ halogen interactions and the different size of the counterion. The occupancies of the fragments were determined by refining them against a free variable with the sum of the two sites set to equal 1 . The occupancies refined to $\sim 74 / 26$ for $\mathrm{Cl} / \mathrm{I}$ and restraints and constraints were applied to maintain sensible geometries and thermal parameters. Crystal structure and refinement data are given in Table S1. Crystallographic data for compounds $\mathbf{1 a}$ and $\mathbf{8}$ have been deposited with the Cambridge Crystallographic Data Centre as supplementary publication 
CCDC 1976972, 1985237. Copies of the data can be obtained free of charge on application to CCDC, 12 Union Road, Cambridge CB2 1EZ, UK [fax $(+44) 1223$ 336033, e-mail: deposit@ccdc.cam.ac.uk].

Table S1: Crystal data and structure refinement for $1 \mathrm{a}$ and 8

Identification code

Empirical formula

Formula weight

Temperature/K

Crystal system

Space group

$a / \AA$

$b / \AA$

$c / \AA$

$\alpha /{ }^{\circ}$

$\beta /{ }^{\circ}$

$\gamma /{ }^{\circ}$

Volume $/ \AA^{3}$

Z

$\rho_{\text {calc }} \mathrm{g} / \mathrm{cm}^{3}$

$\mu / \mathrm{mm}^{-1}$

$\mathrm{F}(000)$

Crystal size $/ \mathrm{mm}^{3}$

Radiation

$2 \theta$ range for data collection $/{ }^{\circ}$

Index ranges

Reflections collected

$\mathrm{R}_{\text {int }} / \mathrm{R}_{\text {sigma }}$
$1 \mathrm{a}$

$\mathrm{C}_{20} \mathrm{H}_{33} \mathrm{BCl}_{0.74} \mathrm{I}_{0.26} \mathrm{NO}_{2}$

389.28

100(2)

monoclinic

$P 2{ }_{1} / c$

$16.8872(12)$

9.0985(6)

$14.5635(10)$

90

102.2370(10)

90

2186.8(3)

4

1.182

0.519

829.0

$0.415 \times 0.342 \times 0.184$

$\operatorname{MoK} \alpha(\lambda=0.71073)$

4.936 to 55.818

$-22 \leq \mathrm{h} \leq 22$,

$-8 \leq \mathrm{k} \leq 11$,

$-18 \leq 1 \leq 19$

19323

$0.0430 / 0.0402$
8

$\mathrm{C}_{17} \mathrm{H}_{36} \mathrm{BNO}_{3}$

313.28

100(2)

monoclinic

$P 2{ }_{1} / c$

9.2394(13)

16.054(2)

13.4313(18)

90

104.686(9)

90

1927.1(5)

4

1.080

0.071

696.0

$0.372 \times 0.263 \times 0.104$

$\operatorname{MoK} \alpha(\lambda=0.71073)$

4.032 to 50.698

$-11 \leq \mathrm{h} \leq 11$,

$-19 \leq \mathrm{k} \leq 19$,

$-15 \leq 1 \leq 16$

22565

0.1213 / 0.0833 


$\begin{array}{lll}\text { Data/restraints/parameters } & 5226 / 152 / 274 & 3522 / 0 / 210 \\ \text { Goodness-of-fit on } \mathrm{F}^{2} & 1.028 & 1.020 \\ \text { Final R indexes [I }>=2 \sigma(\mathrm{I})] & \mathrm{R}_{1}=0.0366, & \mathrm{R}_{1}=0.0548, \\ & \mathrm{wR}_{2}=0.0828 & \mathrm{wR}_{2}=0.1148 \\ \text { Final R indexes [all data] } & \mathrm{R}_{1}=0.0556, & \mathrm{R}_{1}=0.1097, \\ & \mathrm{wR}_{2}=0.0902 & \mathrm{wR}_{2}=0.1371 \\ \text { Largest diff. peak/hole / e } \AA^{-3} & 0.40 /-0.23 & 0.21 /-0.24\end{array}$


Table S2: Comparing ${ }^{13} \mathrm{C}$ NMR data of 1a with other structures.

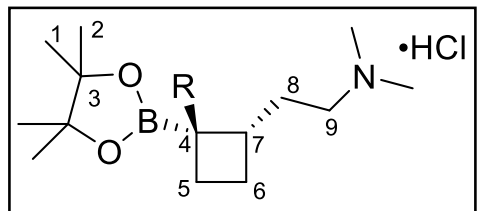

\begin{tabular}{lllllllll}
\hline Compound & C1 & C2 & C3 & C5 & C6 & C7 & C8 & C9 \\
\hline 1a & 24.8 & 24.7 & 83.8 & 29.5 & 25.3 & 42.2 & 29.7 & 56.3 \\
$\mathbf{1 b}$ & 24.8 & 24.7 & 83.7 & 29.5 & 25.2 & 42.5 & 29.6 & 56.3 \\
$\mathbf{1 c}$ & 24.8 & 24.6 & 84.5 & 29.1 & 25.0 & 42.4 & 29.5 & 56.0 \\
$\mathbf{1 d}$ & 24.9 & 24.6 & 83.8 & 28.6 & 24.9 & 42.2 & 28.6 & 56.0 \\
$\mathbf{1 e}$ & 24.8 & 24.7 & 83.9 & 29.3 & 25.2 & 42.5 & 29.6 & 56.2 \\
$\mathbf{1 f}$ & 24.8 & 24.7 & 84.0 & 29.4 & 25.1 & 42.2 & 29.6 & 56.3 \\
$\mathbf{1 g}$ & 24.8 & 24.7 & 83.8 & 29.6 & 25.3 & 42.4 & 29.7 & 56.4 \\
$\mathbf{1 h}$ & 24.8 & 24.7 & 83.9 & 29.6 & 25.3 & 42.3 & 29.8 & 56.4 \\
$\mathbf{1 i}$ & 24.8 & 24.7 & 83.7 & 27.5 & 25.0 & 42.3 & 29.1 & 56.3 \\
$\mathbf{1 j}$ & 24.9 & 24.8 & 83.0 & 27.2 & 25.1 & 44.3 & 27.3 & 58.0 \\
$\mathbf{1 k}$ & 25.3 & 24.9 & 83.5 & 28.9 & 24.6 & 44.2 & 29.8 & 56.4 \\
$\mathbf{1 l}$ & 25.4 & 24.7 & 83.4 & 27.8 & 24.8 & 43.1 & 29.2 & 56.4 \\
$\mathbf{1 m}$ & 25.3 & 25.0 & 83.2 & 26.2 & 24.0 & 40.3 & 29.6 & 56.4 \\
\hline
\end{tabular}

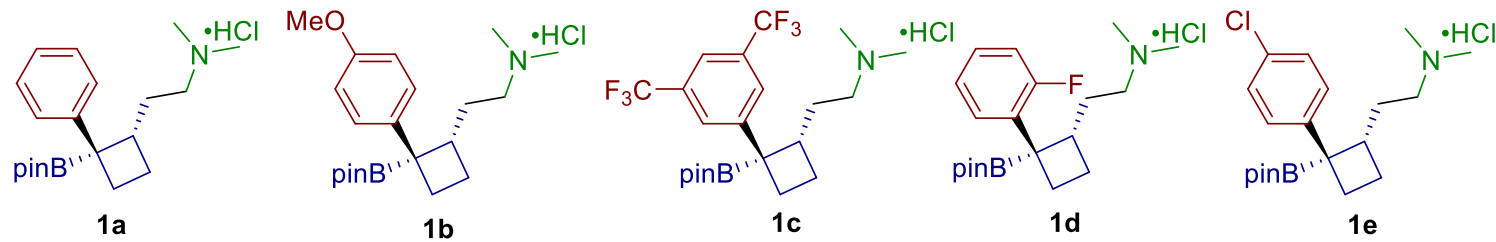

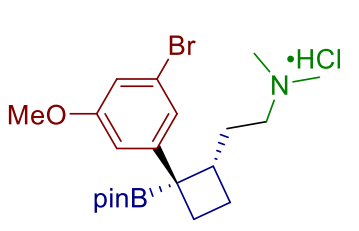

$1 f$

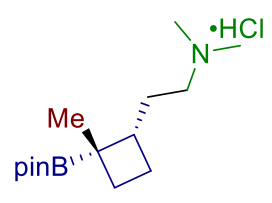

$1 \mathrm{k}$

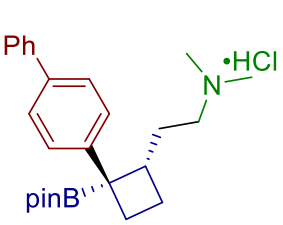

$1 \mathrm{~g}$

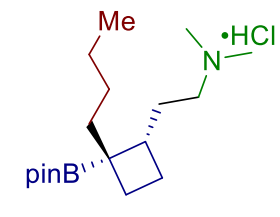

11

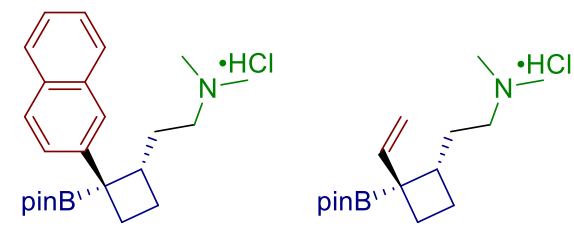

$1 \mathrm{~h}$

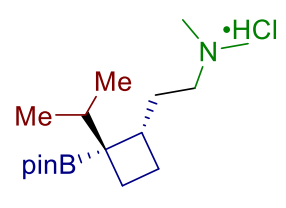


Table S3: Comparing ${ }^{13} \mathrm{C}$ NMR data of 1 ag with other side chain substituted products.

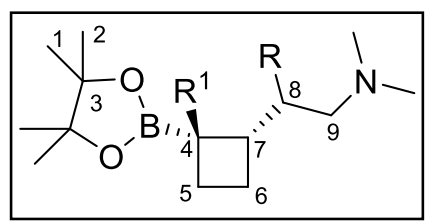

\begin{tabular}{llllllllll}
\hline Compound & C1 & C2 & C3 & C5 & C6 & C7 & C8 & C9 & R \\
\hline 1ag & 25.1 & 24.9 & 82.9 & 29.4 & 23.9 & 52.7 & 36.7 & 63.7 & Me \\
1z & 24.9 & 24.8 & 83.6 & 30.5 & 24.7 & 48.1 & 36.8 & 63.7 & $\mathrm{Me}$ \\
1aa & 25.2 & 24.9 & 83.4 & 30.9 & 24.8 & 47.8 & 41.5 & 62.1 & $\left(\mathrm{CH}_{2}\right)_{3} \mathrm{Me}$ \\
1ab & 25.2 & 24.9 & 83.4 & 30.8 & 24.8 & 48.0 & 41.5 & 62.2 & $\left(\mathrm{CH}_{2}\right)_{2} \mathrm{Ph}$ \\
1ac & 25.1 & 24.6 & 83.4 & 30.8 & 25.4 & 46.2 & 44.2 & 63.4 & $\left(\mathrm{CH}_{2}\right)_{2} \mathrm{OH}$ \\
1ad & 25.3 & 25.0 & 83.1 & 31.7 & 24.6 & 48.6 & 43.2 & 62.0 & $\mathrm{Cypropyl}$ \\
1ae & 24.9 & 24.9 & 83.4 & 28.4 & 25.0 & 45.5 & 46.9 & 62.6 & $\mathrm{Ph}$ \\
\hline
\end{tabular}

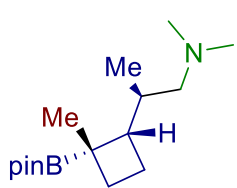

$1 \mathrm{ag}$

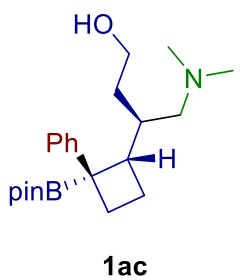

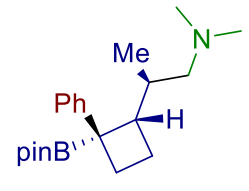

$1 z$

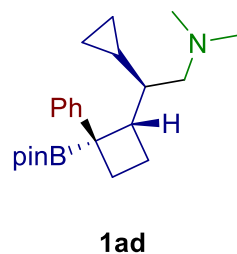

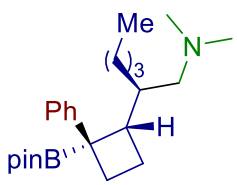

1 aa

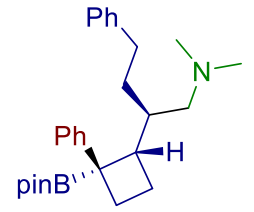

$1 \mathrm{ab}$

These tables show the consistency in ${ }^{13} \mathrm{C}$ data of the cyclobutyl boronic ester products implicating that they are all the same diastereoisomer. 


\section{GC-MS of 1a and 2a}
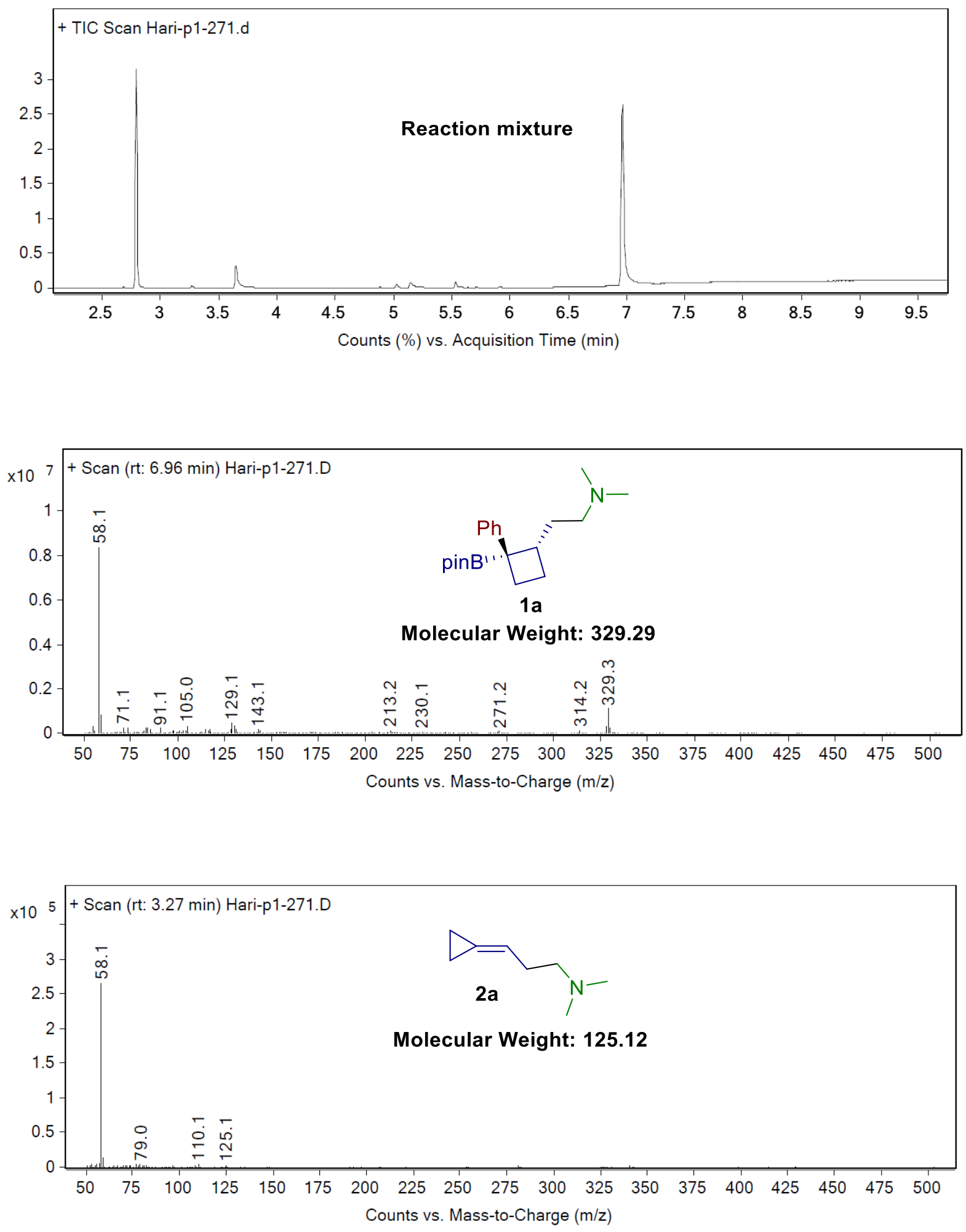


\section{References}

1. Harris, M. R.; Wisniewska, H. M.; Jiao, W.; Wang, X.; Bradow, J. N. A Modular Approach to the Synthesis of Gem-Disubstituted Cyclopropanes. Org. Lett. 2018, 20, 2867.

2. Synthesized using the procedure reported in the reference: Fabre, J. M.; Garín, J.; Uriel, S. The Synthesis of Primary, Secondary and Tertiary Aminomethyltetrathiafulvalenes. Tetrahedron 1992, 48, 3983.

3. Slutskyy, Y.; Jamison, C. R.; Zhao, P.; Lee, J.; Rhee, Y. H.; Overman, L. E. Versatile Construction of 6-Substituted Cis-2,8-Dioxabicyclo[3.3.0]Octan-3-Ones: Short Enantioselective Total Syntheses of Cheloviolenes A and B and Dendrillolide C. J. Am. Chem. Soc. 2017, 139, 7192.

4. Gaussian 09, Revision C1, Frisch, M. J.; Trucks, G. W.; Schlegel, H. B.; Scuseria, G. E.; Robb, M. A.; Cheeseman, J. R.; Scalmani, G.; Barone, V.; Mennucci, B.; Petersson, G. A.; Nakatsuji, H.; Caricato, M.; Li, X.; Hratchian, H. P.; Izmaylov, A. F.; Bloino, J.; Zheng, G.; Sonnenberg, J. L.; Hada, M.; Ehara, M.; Toyota, K.; Fukuda, R.; Hasegawa, J.; Ishida, M.; Nakajima, T.; Honda, Y.; Kitao, O.; Nakai, H.; Vreven, T.; Montgomery, Jr., J. A.; Peralta, J. E.; Ogliaro, F.; Bearpark, M.; Heyd, J. J.; Brothers, E.; Kudin, K. N.; Staroverov, V. N.; Kobayashi, R.; Normand, J.; Raghavachari, K.; Rendell, A.; Burant, J. C.; Iyengar, S. S.; Tomasi, J.; Cossi, M.; Rega, N.; Millam, J. M.; Klene, M.; Knox, J. E.; Cross, J. B.; Bakken, V.; Adamo, C.; Jaramillo, J.; Gomperts, R.; Stratmann, R. E.; Yazyev, O.; Austin, A. J.; Cammi, R.; Pomelli, C.; Ochterski, J. W.; Martin, R. L.; Morokuma, K.; Zakrzewski, V. G.; Voth, G. A.; Salvador, P.; Dannenberg, J. J.; Dapprich, S.; Daniels, A. D.; Farkas, Ö.; Foresman, J. B.; Ortiz, J. V.; Cioslowski, J.; Fox, D. J. Gaussian, Inc., Wallingford CT, 2009. 5. http://comp.chem.umn.edu/info/DFT.htm.

6. Bruker, SAINT+ v8.38A Integration Engine, Data Reduction Software, Bruker Analytical X-ray Instruments Inc., Madison, WI, USA 2015.

7. Bruker, SADABS 2014/5, Bruker AXS area detector scaling and absorption correction, Bruker Analytical X-ray Instruments Inc., Madison, Wisconsin, USA 2014/5.

8. Sheldrick, G. M., SHELXT - Integrated space-group and crystal-structure determination. Acta Crystallogr., Sect. A 2015, 71, 3.

9. (a) Sheldrick, G. M., A short history of SHELX. Acta Crystallogr., Sect. A 2008, 64, 112. (b) Sheldrick, G. M., Crystal structure refinement with SHELXL. Acta Crystallogr., Sect. C 2015, 71, 3. 
10. Dolomanov, O. V.; Bourhis, L. J.; Gildea, R. J.; Howard, J. A. K.; Puschmann, H., OLEX2: a complete structure solution, refinement and analysis program. J. Appl. Crystallogr. 2009, 42, 339. 